\title{
Risk Impact of BWR Technical Specifications Requirements During Shutdown
}

Manuscript Completed: September 1994

Date Published: October 1994

Prepared by

B. D. Staple, H. K. Kirk, Sandia National Laboratories

J. Yakle, Science Applications International Corporation

Sandia National Laboratories

Albuquerque, NM 87185

Science Applications International Corporation

2109 Air Park Road S. E.

Albuquerque, NM 87106

Prepared for

Division of Systems Research

Office of Nuclear Regulatory Research

U.S. Nuclear Regulatory Commission

Washington, DC 20555-0001

Under Memorandum of Understanding DOE 40-550-75

NRC FIN L1830 


\section{DISCLAIMER}

This report was prepared as an account of work sponsored by an agency of the United States Government. Neither the United States Government nor any agency thereof, nor any of their employees, make any warranty, express or implied, or assumes any legal liability or responsibility for the accuracy, completeness, or usefulness of any information, apparatus, product, or process disclosed, or represents that its use would not infringe privately owned rights. Reference herein to any specific commercial product, process, or service by trade name, trademark, manufacturer, or otherwise does not necessarily constitute or imply its endorsement, recommendation, or favoring by the United States Government or any agency thereof. The views and opinions of authors expressed herein do not necessarily state or reflect those of the United States Government or any agency thereof. 


\section{DISCLAIMER}

Portions of this document may be illegible in electronic image products. Images are produced from the best available original document. 


\begin{abstract}
This report presents an application of probabilistic models and risk based criteria for determining the risk impact of the Limiting Conditions of Operations (LCOs) in the Technical Specifications (TSs) of a boiling water reactor during shutdown. This analysis studied the risk impact of the current

requirements of Allowed Outage Times (AOTs) and Surveillance Test Intervals (STIs) in eight Plant Operational States (POSs) which encompass power operations, shutdown, and refueling. This report also discusses insights concerning TS action statements.
\end{abstract}





\section{Contents}

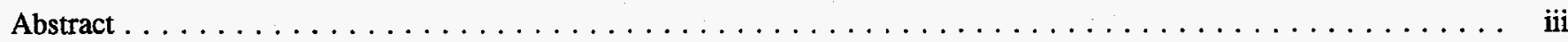

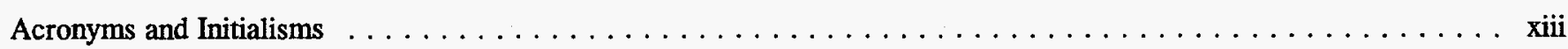

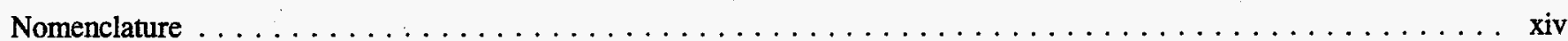

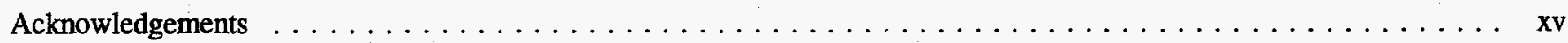

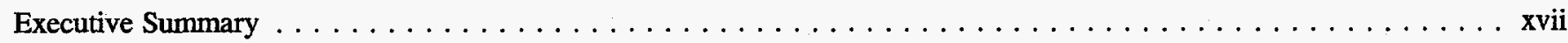

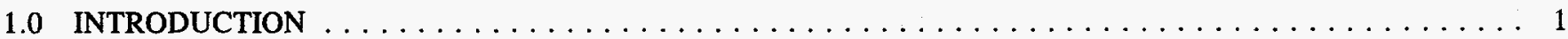

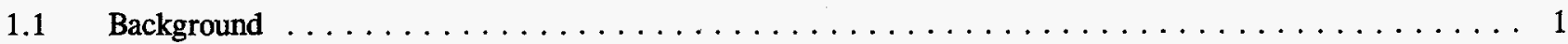

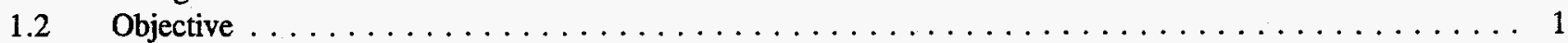

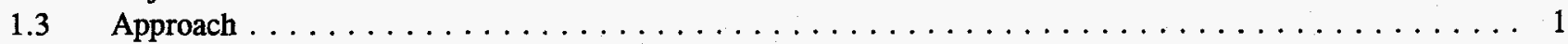

1.4 Report Organization $\ldots \ldots \ldots \ldots \ldots \ldots \ldots \ldots \ldots \ldots \ldots \ldots \ldots \ldots \ldots \ldots \ldots \ldots \ldots$

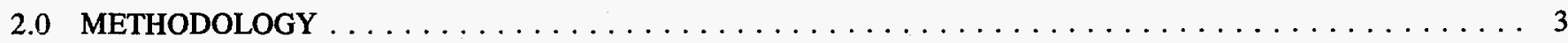

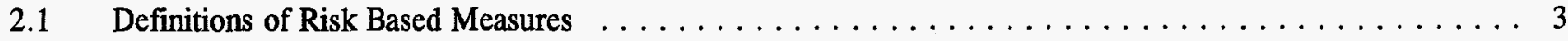

2.1.1 Definitions of Risk Measures for Evaluating the Impact of Maintenance Activities $\ldots \ldots \ldots \ldots \ldots 3$

2.1.1.1 Risk Measures of Allowed Outage Times for Single Systems $\ldots \ldots \ldots \ldots \ldots \ldots \ldots \ldots$

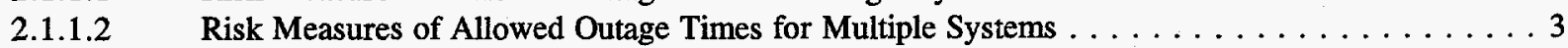

2.1.2 Definitions of Risk Measures for Evaluating the Impact of Surveillance Test Activities . . . . . . . 4

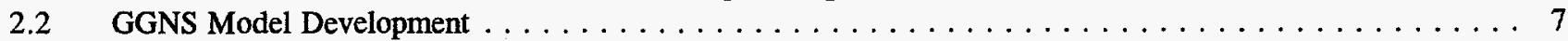

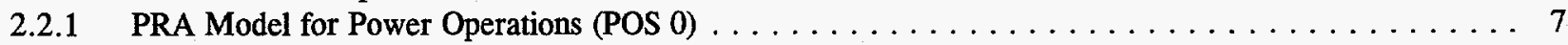

2.2.2 PRA Model for Low Power Operations $($ POS 1$) \ldots \ldots \ldots \ldots \ldots \ldots \ldots \ldots \ldots \ldots \ldots$

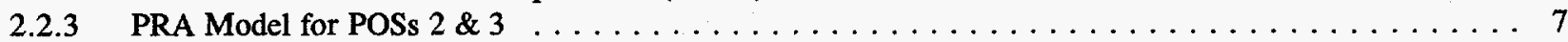

2.2.4 PRA Model for POS $4 \ldots \ldots \ldots \ldots \ldots \ldots \ldots \ldots \ldots \ldots \ldots \ldots \ldots \ldots \ldots$

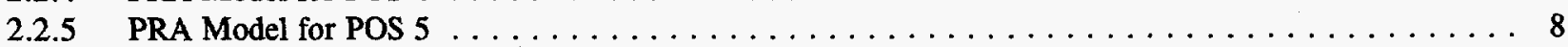

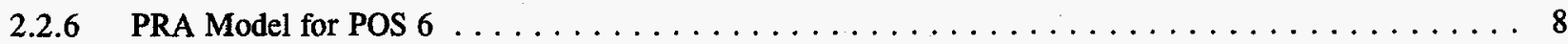

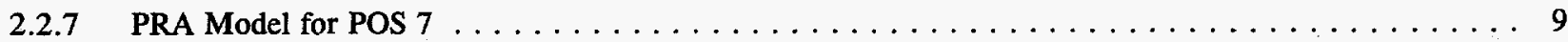

2.3 Model Evaluations $\ldots \ldots \ldots \ldots \ldots \ldots \ldots \ldots \ldots \ldots \ldots \ldots \ldots \ldots \ldots \ldots \ldots \ldots \ldots$

3.0 RESULTS OF THE RISK IMPACT OF MAINTENANCE ACTIVITIES $\ldots \ldots \ldots \ldots \ldots \ldots \ldots \ldots \ldots \ldots$

$3.1 \quad$ Risk Impact of Emergency Diesel Generator (EDG) Maintenance Outage $\ldots \ldots \ldots \ldots \ldots \ldots \ldots \ldots$

3.1.1 Single Train Maintenance on the EDGs $\ldots \ldots \ldots \ldots \ldots \ldots \ldots \ldots \ldots \ldots \ldots \ldots \ldots$

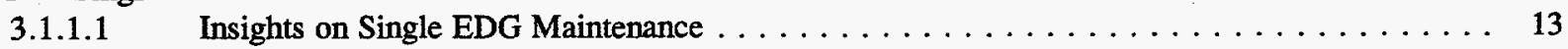

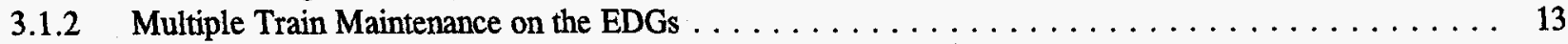

3.1.2.1 Insights on Multiple EDG Maintenance $\ldots \ldots \ldots \ldots \ldots \ldots \ldots \ldots \ldots \ldots \ldots \ldots$

3.2 Risk Impact of Standby Service Water (SSW) Maintenance Outage $\ldots \ldots \ldots \ldots \ldots \ldots \ldots \ldots \ldots \ldots$

3.2.1 Single Train Maintenance on the $\mathrm{SSW} \ldots \ldots \ldots \ldots \ldots \ldots \ldots \ldots \ldots \ldots \ldots \ldots \ldots \ldots$

3.2.1.1 Insights on Single SSW Train Maintenance $\ldots \ldots \ldots \ldots \ldots \ldots \ldots \ldots \ldots \ldots$

3.2.2 Multiple Train Maintenance on SSW A \& SSW C $\ldots \ldots \ldots \ldots \ldots \ldots \ldots \ldots \ldots \ldots \ldots \ldots$

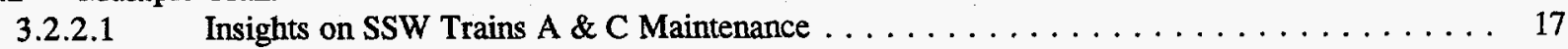

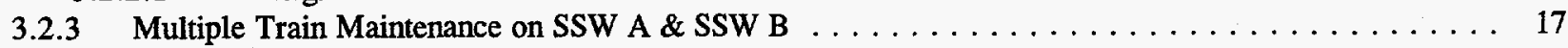

3.2.3.1 Insights on SSW Trains A \& B Maintenance $\ldots \ldots \ldots \ldots \ldots \ldots \ldots \ldots \ldots \ldots \ldots \ldots$

3.3 Risk Impact of DC Power Batteries (BATTs) Maintenance Outage $\ldots \ldots \ldots \ldots \ldots \ldots \ldots \ldots \ldots$

3.3.1 Single Train Maintenance on the BATTs $\ldots \ldots \ldots \ldots \ldots \ldots \ldots \ldots \ldots \ldots \ldots \ldots$

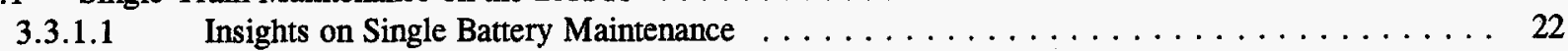

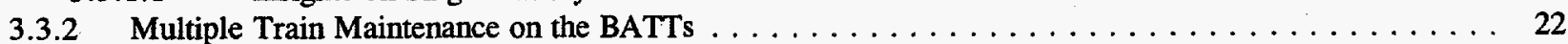

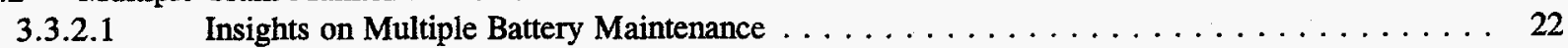

3.4 Risk Impact of Emergency Core Cooling System (ECCS) Maintenance Outage $\ldots \ldots \ldots \ldots \ldots \ldots$ 


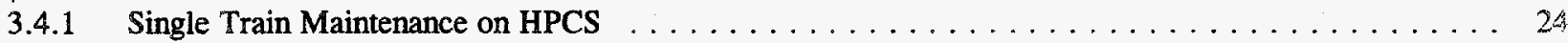

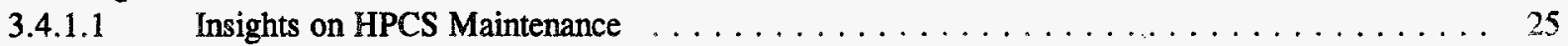

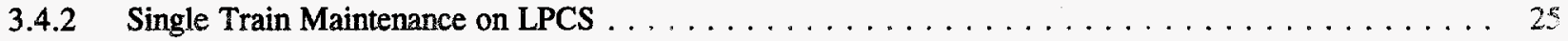

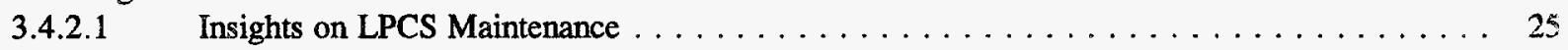

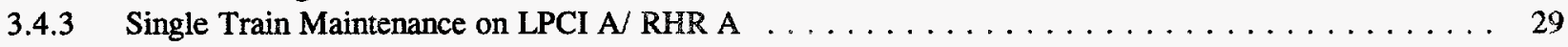

3.4.3.1 Insights on LPCI A/ RHR A Maintenance $\ldots \ldots \ldots \ldots \ldots \ldots \ldots \ldots \ldots \ldots \ldots$

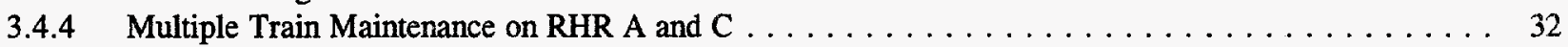

3.4.4.1 Insights on Multiple Train RHR A \& C Maintenance $\ldots \ldots \ldots \ldots \ldots \ldots \ldots \ldots \ldots$

$3.5 \quad$ Risk Impact of Reactor Core Isolation Cooling (RCIC) Maintenance Outage $\ldots \ldots \ldots \ldots \ldots \ldots \ldots$

3.5.1 Single Train Maintenance on the RCIC $\ldots \ldots \ldots \ldots \ldots \ldots \ldots \ldots \ldots \ldots \ldots \ldots \ldots \ldots \ldots \ldots$

3.5.1.1 Insights on RCIC Maintenance . . . . . . . . . . . . . . . . . 33

3.6 Risk Impact of Suppression Pool (ISSP) Drainage and Safety Relief Valves (SRVs) Maintenance Outage $\ldots .35$

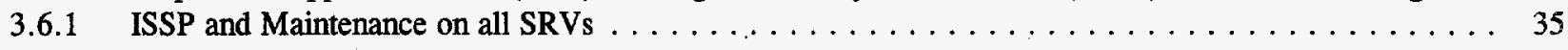

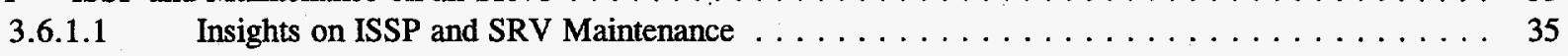

4.0 RESULTS OF THE RISK IMPACT OF SURVEILLANCE TEST INTERVALS $\ldots \ldots \ldots \ldots \ldots \ldots \ldots$

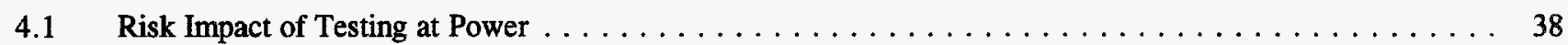

4.1.1 Emergency Diesel Generator (EDG) Surveillance $\ldots \ldots \ldots \ldots \ldots \ldots \ldots \ldots \ldots \ldots \ldots \ldots$

4.1.2 Emergency Core Cooling System (ECCS) Surveillance $\ldots \ldots \ldots \ldots \ldots \ldots \ldots \ldots \ldots \ldots \ldots$

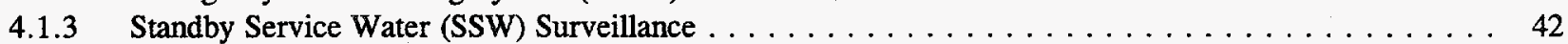

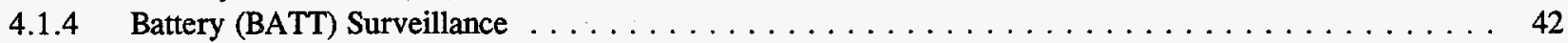

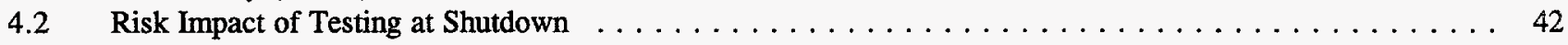

4.2.1 Emergency Diesel Generator (EDG) Surveillance $\ldots \ldots \ldots \ldots \ldots \ldots \ldots \ldots \ldots \ldots \ldots \ldots \ldots$

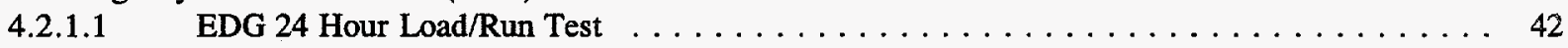

4.2.1.2 EDG 18 Month Functional (LOP) Test $\ldots \ldots \ldots \ldots \ldots \ldots \ldots \ldots \ldots \ldots \ldots$

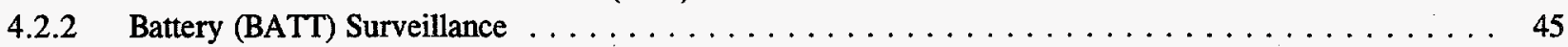

4.2.3 Emergency Core Cooling System (ECCS) Surveillance $\ldots \ldots \ldots \ldots \ldots \ldots \ldots \ldots \ldots \ldots$

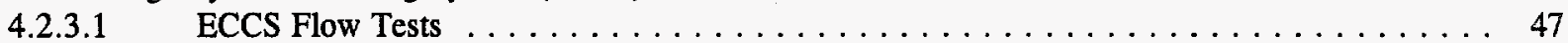

4.2.3.2 ECCS Logic System Functional Test $\ldots \ldots \ldots \ldots \ldots \ldots \ldots \ldots \ldots \ldots \ldots \ldots$

4.2.3.3 ECCS 18 Month Valve Stroke Test $\ldots \ldots \ldots \ldots \ldots \ldots \ldots \ldots \ldots \ldots \ldots \ldots \ldots$

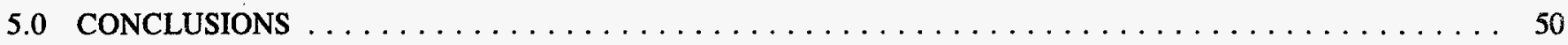

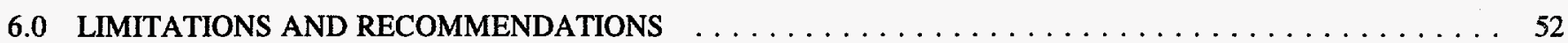

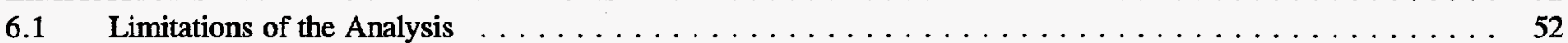

6.2 Recommendations for Model Improvement and Further Study $\ldots \ldots \ldots \ldots \ldots \ldots \ldots \ldots \ldots \ldots$

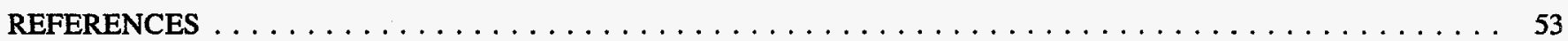

APPENDIX A RAW DATA FOR EVALUATING RISK IMPACT OF SINGLE AND MULTIPLE MAINTENANCE

A.1 Analysis Performed for Each System or Subsystem Out for Maintenance $\ldots \ldots \ldots \ldots \ldots \ldots \ldots \ldots$ A1

A.1.1 Plant Configurations for Single Train Maintenance $\ldots \ldots \ldots \ldots \ldots \ldots \ldots \ldots \ldots \ldots \ldots \ldots$

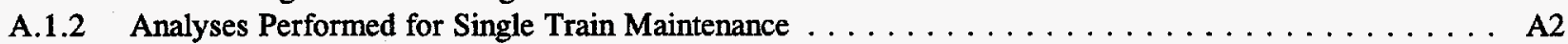

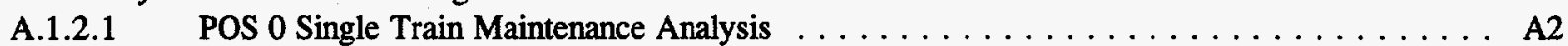

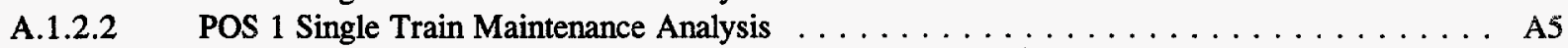

A.1.2.3 POS $2 / 3$ Single Train Maintenance Analysis $\ldots \ldots \ldots \ldots \ldots \ldots \ldots \ldots \ldots \ldots \ldots \ldots$

A.1.2.4 POS 4 Single Train Maintenance Analysis $\ldots \ldots \ldots \ldots \ldots \ldots \ldots \ldots \ldots \ldots \ldots \ldots \ldots \ldots$

A.1.2.5 POS 5 Single Train Maintenance Analysis $\ldots \ldots \ldots \ldots \ldots \ldots \ldots \ldots \ldots \ldots \ldots$

A.1.2.6 POS 6 Single Train Maintenance Analysis $\ldots \ldots \ldots \ldots \ldots \ldots \ldots \ldots \ldots \ldots$ A9

A.1.2.7 POS 7 Single Train Maintenance Analysis $\ldots \ldots \ldots \ldots \ldots \ldots \ldots \ldots \ldots \ldots \ldots$

A.1.3 Multiple Train Maintenance Analysis $\ldots \ldots \ldots \ldots \ldots \ldots \ldots \ldots \ldots \ldots \ldots \ldots \ldots$ 
APPENDIX B RAW DATA FOR EVALUATING RISK IMPACT OF SURVEILLANCE TEST ACTIVITIES . . . . . Bi

B.1 Analysis Used to Evaluate the Risk Impact of Performing Surveillance Tests . . . . . . . . . . . . B B

B.2 Raw Data Used for Evaluating the Risk Impact of Surveillance Tests $\ldots \ldots \ldots \ldots$

APPENDIX C RISK IMPACT OF SINGLE AND MULTIPLE MAINTENANCE ACTIVITIES . . . . . . . . . . . Ci

APPENDIX D RISK IMPACT OF SURVEILLANCE ACTIVITIES $\ldots \ldots \ldots \ldots \ldots$

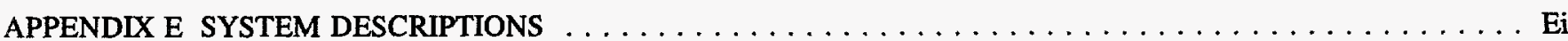

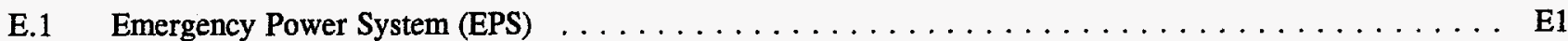

E.1.1 EPS System Description $\ldots \ldots \ldots \ldots \ldots \ldots \ldots \ldots \ldots \ldots \ldots \ldots \ldots$

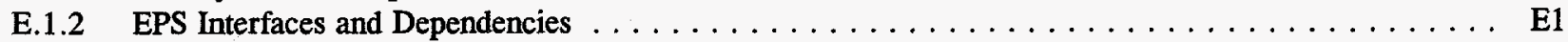

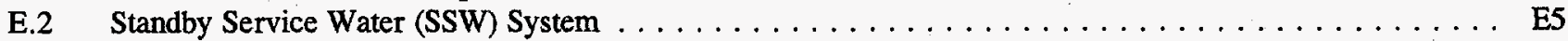

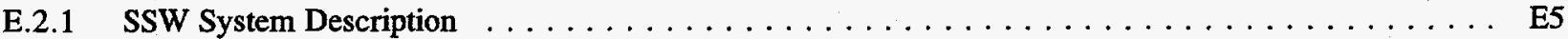

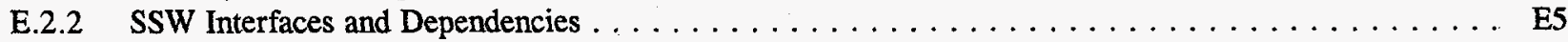

E.3 High Pressure Core Spray System (HPCS) $\ldots \ldots \ldots \ldots \ldots \ldots$

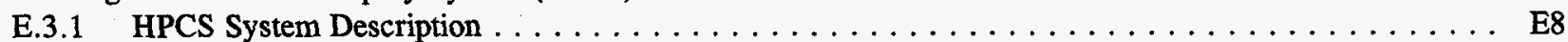

E.3.2 HPCS Interfaces and Dependencies $\ldots \ldots \ldots \ldots \ldots \ldots \ldots \ldots$

E.4 Low Pressure Core Spray (LPCS) System . . . . . . . . . . . . . . . . . . . . . . . . . E14

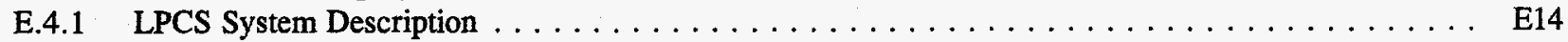

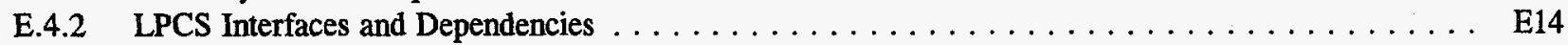

E.5 Low Pressure Coolant Injection (LPCI) System $\ldots \ldots \ldots \ldots \ldots \ldots \ldots \ldots$

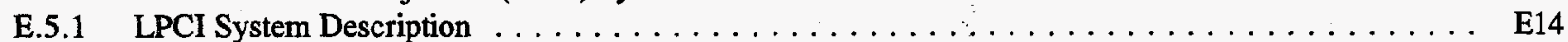

E.5.2 LPCI Interfaces and Dependencies $\ldots \ldots \ldots \ldots \ldots \ldots \ldots \ldots \ldots \ldots$

E.6 Alternate Decay Heat Removal (ADHR) System $\ldots \ldots \ldots \ldots \ldots \ldots \ldots$

E.6.1 ADHR System Description $\ldots \ldots \ldots \ldots \ldots \ldots \ldots \ldots \ldots \ldots$

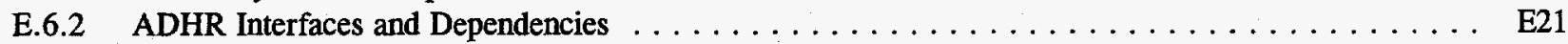

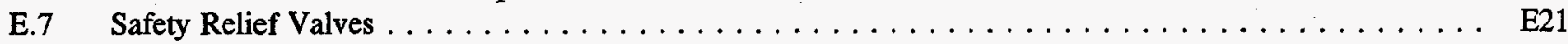

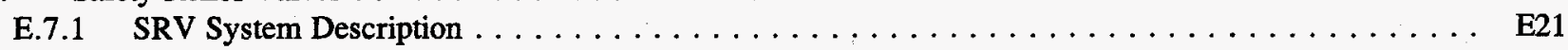

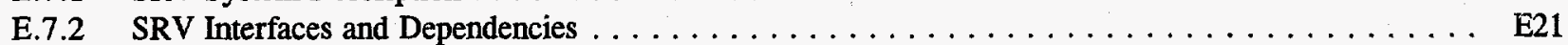


Contents

\section{List of Figures}

1. Change in Core Damage Frequency for Taking an EDG Out for Maintenance $\ldots \ldots \ldots \ldots \ldots \ldots$ xviii

2. Change in Core Damage Frequency for Taking a Single EDG Out for Maintenance During the Different POSs in an

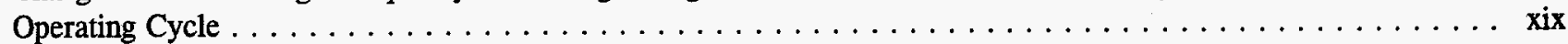

3. Increase in Conditional CDF for Taking a Single EDG Out of Service During the Different POSs in an Operating

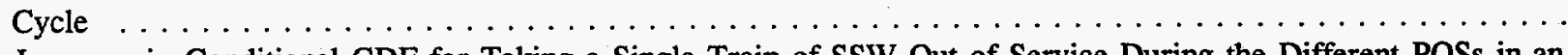

4. Increase in Conditional CDF for Taking a Single Train of SSW Out of Service During the Different POSs in an

5. Increase in Conditional $\mathrm{CDF}$ for Taking a Single Battery Out of Service During the Different POSs in an Operating Cycle

6. Increase in Conditional CDF for Taking HPCS Out of Service During the Different POSs in an Operating Cycle .

7. Increase in Conditional CDF for Taking LPCS Out of Service During the Different POSs in an Operating Cycle . .

8. Increase in Conditional CDF for Taking LPCI A Out of Service During the Different POSs in an Operating Cycle

9. Increase in Conditional Core Damage Frequency for Single EDG Maintenance $\ldots \ldots \ldots \ldots \ldots \ldots \ldots$

10. Change in Core Damage Frequency for Taking a Single EDG Out for Maintenance During the Different POSs in an Operating Cycle

11. Increase in Conditional Core Damage Frequency for Multiple EDG Maintenance

12. Increase in Conditional Core Damage Frequency for Single Train SSW Maintenance . . . . . . . . . . . . . . . . . .

13. Change in Core Damage Frequency for Taking a Single Train of SSW Out for Maintenance During the Different POSs

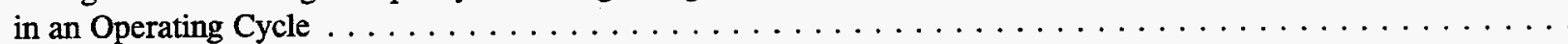

14. Increase in Conditional Core Damage Frequency for Multiple Train SSW Maintenance $\ldots \ldots \ldots \ldots \ldots \ldots \ldots$

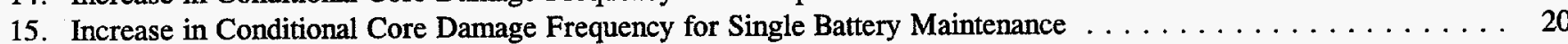

16. Change in Core Damage Frequency for Taking a Single Battery Out for Maintenance During the Different POSs in an Operating Cycle

17. Increase in Conditional Core Damage Frequency for Multiple Battery Maintenance

18. Increase in Conditional Core Damage Frequency for HPCS Maintenance

19. Change in Core Damage Frequency for Taking HPCS Out for Maintenance During the Different POSs in an Operating Cycle

20. Increase in Conditional Core Damage Frequency for LPCS Maintenance

21. Change in Core Damage Frequency for Taking LPCS Out for Maintenance During the Different POSs in an Operating Cycle

22. Increase in Conditional Core Damage Frequency for LPCI A/ RHR A Maintenance $\ldots \ldots \ldots \ldots \ldots \ldots \ldots$

23. Change in Core Damage Frequency for Taking LPCI A Out for Mointenance During the Different POSs in an Operating Cycle.

24. Increase in Conditional Core Damage Frequency for Multiple Train RHR Maintenance $\ldots \ldots \ldots \ldots \ldots$

25. Increase in Conditional Core Damage Frequency for RCIC Maintenance $\ldots \ldots \ldots \ldots \ldots \ldots \ldots$

26. Increase in Conditional Core Damage Frequency for Suppression Pool Drainage . . . . . . . . . . . . .

27. Increase in Conditional Core Damage Frequency for Simultaneous Maintenance on SRVs $\ldots \ldots \ldots \ldots \ldots \ldots$

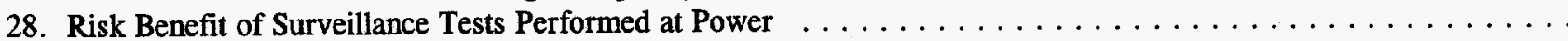

29. Risk Benefit Sensitivities to Different EDG Surveillance Test Arrangements . . . . . . . . . . . . . .

30. Risk Benefit Sensitivities to Different ECCS Surveillance Test Arrangements $\ldots \ldots \ldots \ldots \ldots \ldots \ldots \ldots \ldots$

31. Risk Impact of the EDG 24 Hour Load/Run Test $\ldots \ldots \ldots \ldots \ldots \ldots \ldots \ldots \ldots \ldots \ldots \ldots \ldots$

32. Risk Impact of the EDG 18 Month Functional (LOP) Test $\ldots \ldots \ldots \ldots \ldots \ldots \ldots \ldots \ldots \ldots \ldots \ldots \ldots$

33. Risk Impact of the 18 Month Battery Emergency Load Discharge Test $\ldots \ldots \ldots \ldots \ldots \ldots \ldots \ldots \ldots \ldots \ldots$

34. Risk Impact of the HPCS Quarterly Flow Test $\ldots \ldots \ldots \ldots \ldots \ldots \ldots \ldots \ldots \ldots \ldots \ldots \ldots \ldots \ldots$

35. Risk Impact of the LPCS 18 Month Logic System Functional Test $\ldots \ldots \ldots \ldots \ldots \ldots \ldots \ldots \ldots \ldots \ldots$

36. Risk Impact of the LPCI A 18 Month Stroke and Set Limits Test $\ldots \ldots \ldots \ldots \ldots \ldots \ldots \ldots \ldots \ldots \ldots$

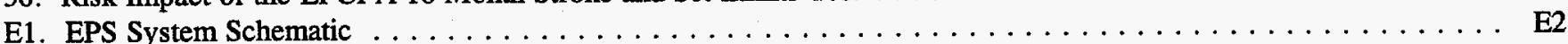

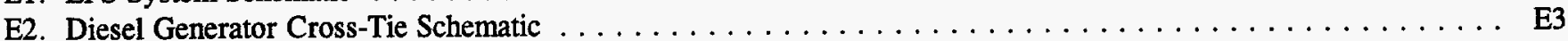

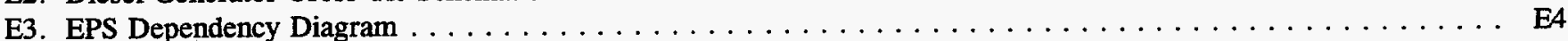

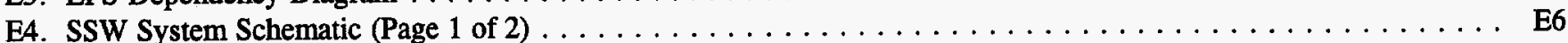

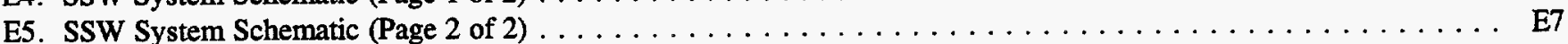




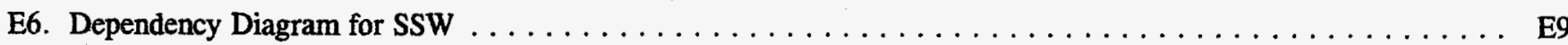

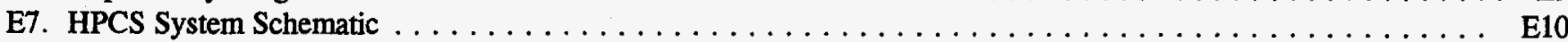

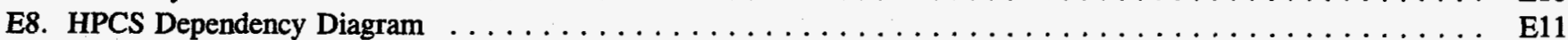

E9. System Actuation Dependency Diagram (Page 1 of 2$) \ldots \ldots \ldots \ldots \ldots$

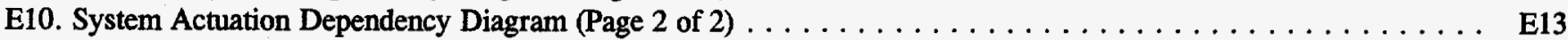

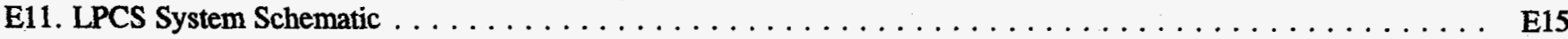

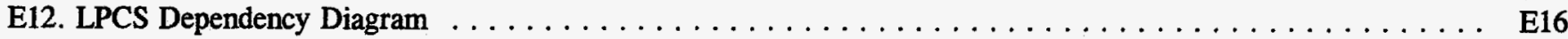

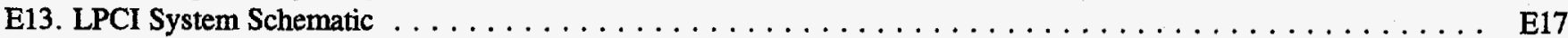

E14. LPCI Dependency Diagram . . . . . . . . . . . . . . . . . . . . . . . . . E19

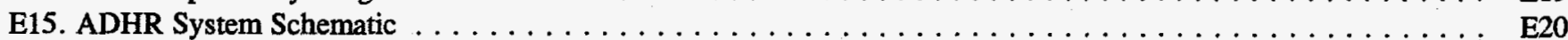

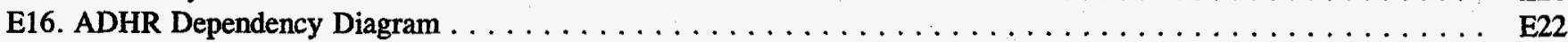

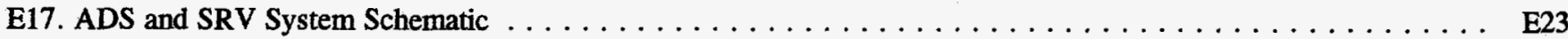

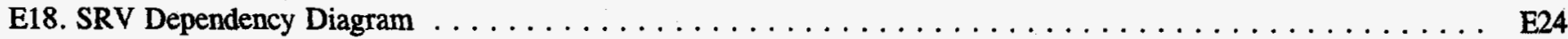


Contents

\section{List of Tables}

1. Time Spent in Each POS in Transition to Refueling $\ldots \ldots \ldots \ldots \ldots \ldots \ldots \ldots \ldots \ldots \ldots \ldots \ldots \ldots$

2. Generic Maintenance Frequencies $\ldots \ldots \ldots \ldots \ldots \ldots \ldots \ldots \ldots \ldots \ldots \ldots \ldots \ldots \ldots \ldots$

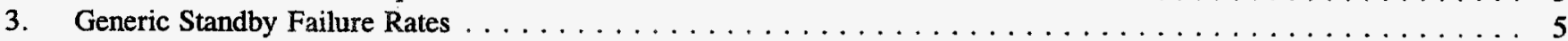

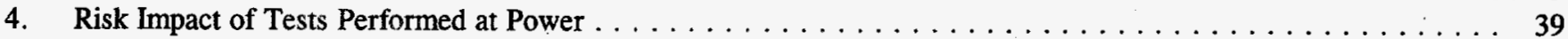

A.1 Single Train Maintenance Results for POSs $0,1,2$, and $3 \ldots \ldots \ldots \ldots \ldots \ldots \ldots \ldots \ldots \ldots \ldots \ldots$

A.2 Single Train Maintenance Results for POSs $4,5,6$, and $7 \ldots \ldots \ldots \ldots \ldots \ldots \ldots \ldots \ldots \ldots \ldots \ldots$

A.3 Multiple Train Maintenance Results for POSs $0,1,2$, and $3 \ldots \ldots \ldots \ldots \ldots \ldots \ldots \ldots \ldots \ldots \ldots \ldots$

A.4 Multiple Train Maintenance Results for POSs $4,5,6$, and $7 \ldots \ldots \ldots \ldots \ldots \ldots \ldots \ldots \ldots \ldots \ldots \ldots$

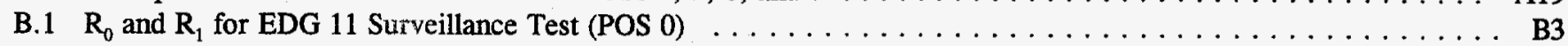

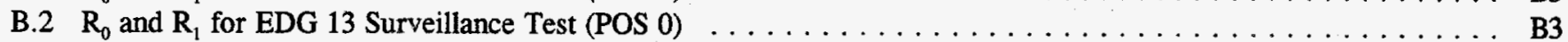

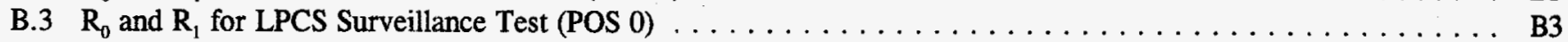

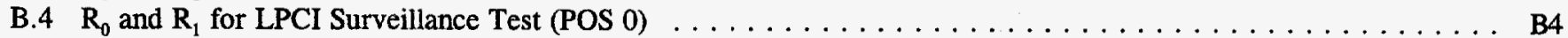

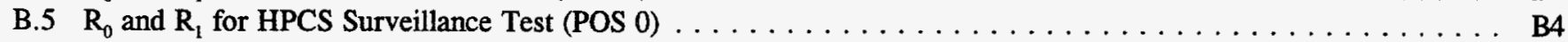

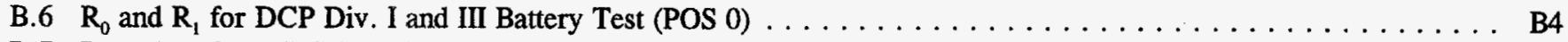

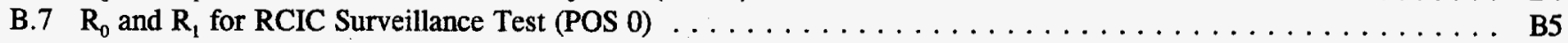

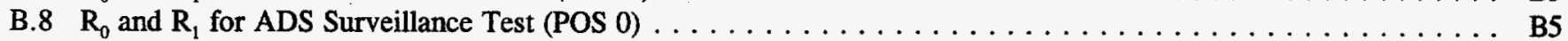

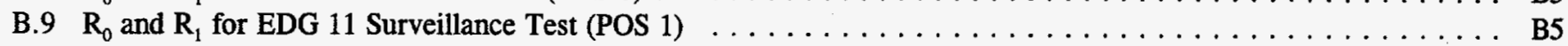

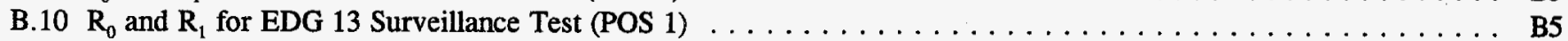

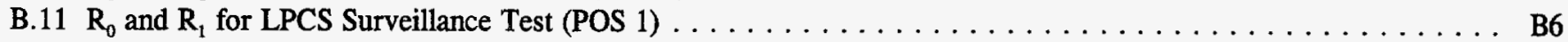

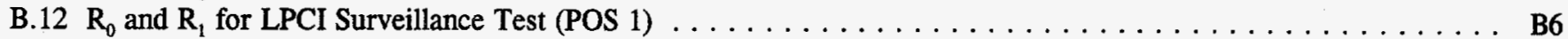

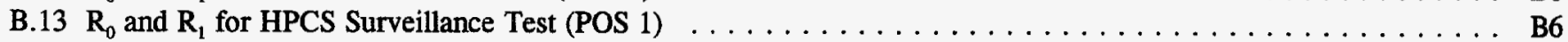

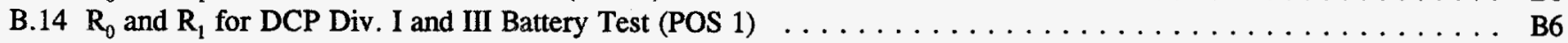

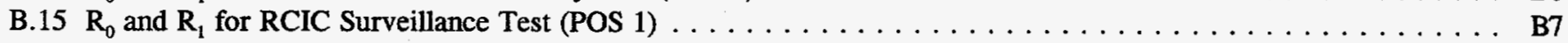

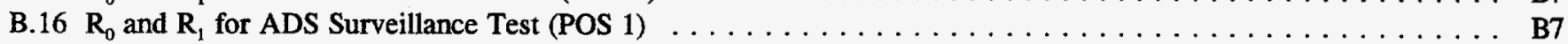

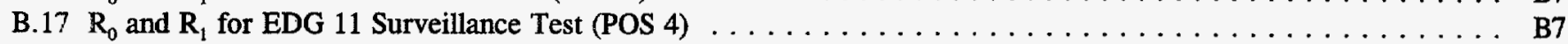

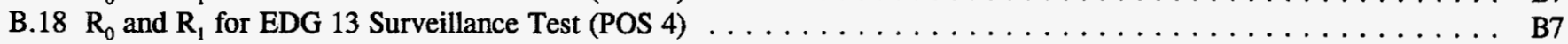

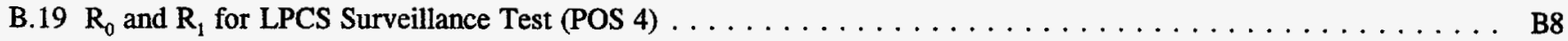

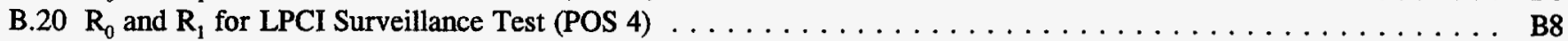

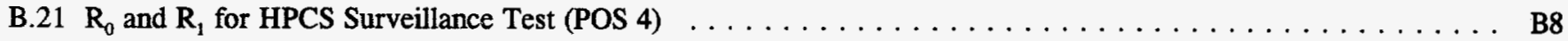

B.22 $R_{0}$ and $R_{1}$ for DCP Div. I and III Battery Test (POS 4) $\ldots \ldots \ldots \ldots \ldots \ldots \ldots \ldots \ldots \ldots \ldots \ldots \ldots$

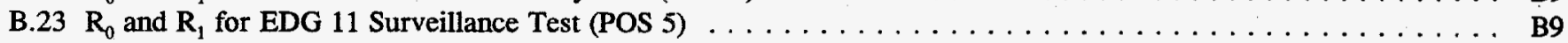

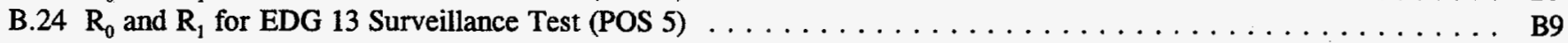

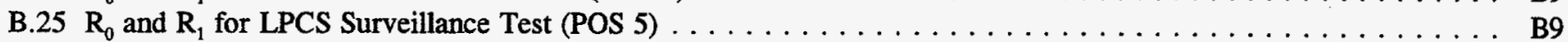

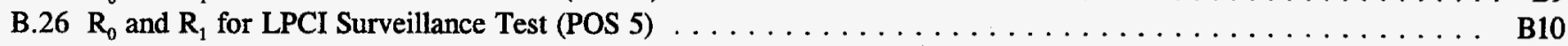

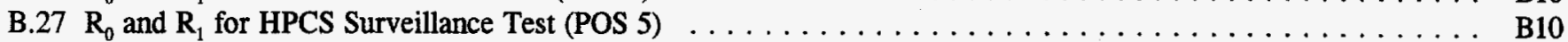

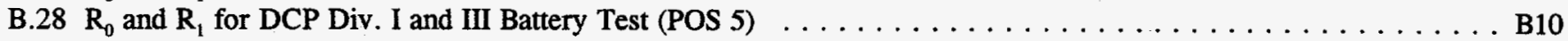

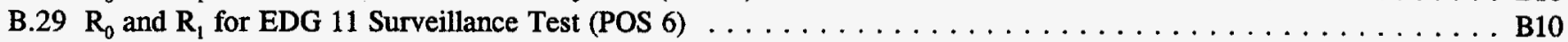

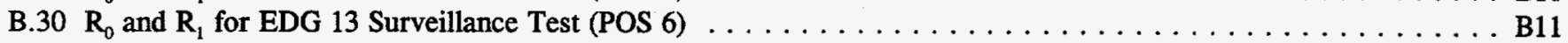

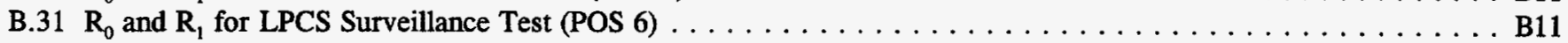

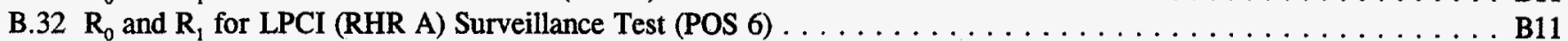

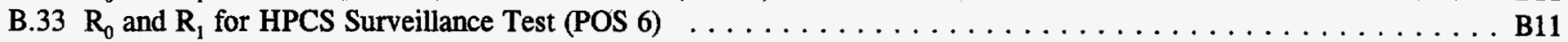

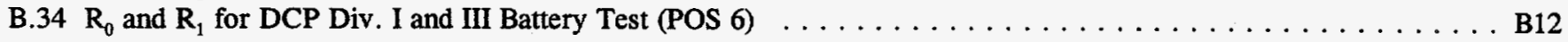

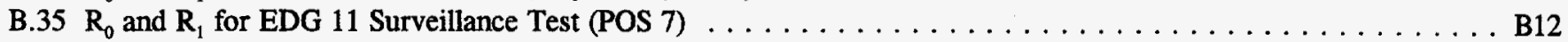

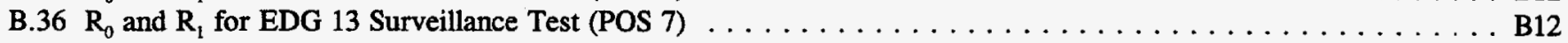

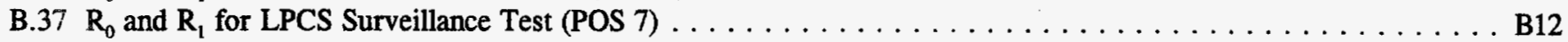

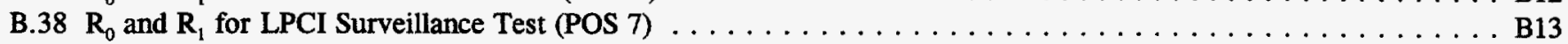

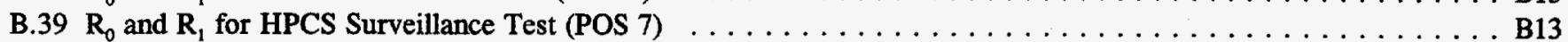

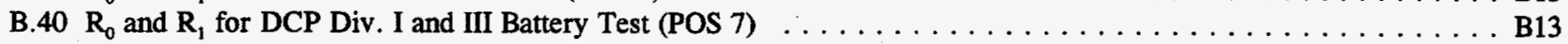

C.1. Impact of Single Component Unavailability at Power $(\mathrm{POS} 0) \ldots \ldots \ldots \ldots \ldots \ldots \ldots \ldots \ldots \ldots \ldots \ldots$

C.2. Impact of Multiple Component Unavailability at Power $(\mathrm{POS} 0) \ldots \ldots \ldots \ldots \ldots \ldots \ldots \ldots \ldots \ldots \ldots$

C.3. Impact of Single Component Unavailability at Low Power $(\operatorname{POS} 1) \ldots \ldots \ldots \ldots \ldots \ldots \ldots \ldots \ldots$ 
C.4. Impact of Multiple Component Unavailability at Low Power $(\operatorname{POS} 1) \ldots \ldots \ldots \ldots \ldots \ldots \ldots \ldots \ldots$ C5

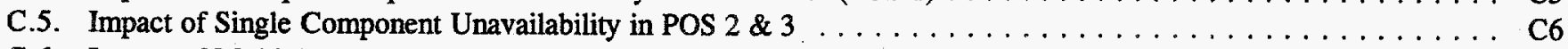

C.6. Impact of Multiple Component Unavailability in POS $2 \& 3 \ldots \ldots \ldots \ldots \ldots \ldots \ldots \ldots \ldots \ldots \ldots \ldots$

C.7. Impact of Single Component Unavailability in POS $4 \ldots \ldots \ldots \ldots \ldots \ldots \ldots \ldots \ldots \ldots \ldots \ldots \ldots$

C.8. Impact of Multiple Component Unavailability in POS $4 \ldots \ldots \ldots \ldots \ldots \ldots \ldots \ldots \ldots \ldots \ldots \ldots \ldots$ C9

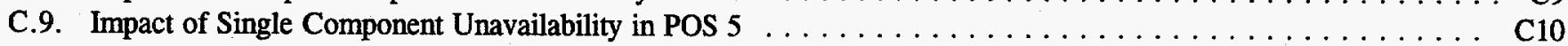

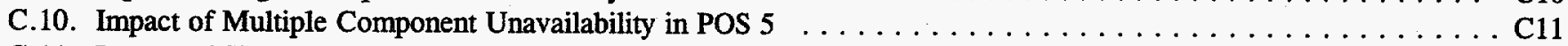

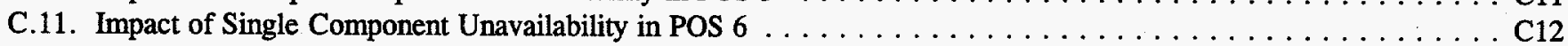

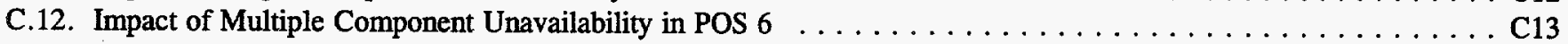

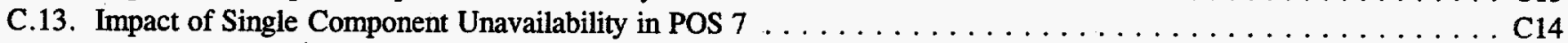

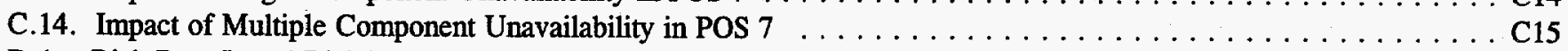

D.1. Risk Benefit and Risk Penalty Due to Equipment Downtime $\ldots \ldots \ldots \ldots \ldots \ldots \ldots \ldots \ldots \ldots \ldots \ldots$ 



\section{Acronyms and Initialisms}

$\begin{array}{ll}\text { ADHRS } & \text { Alternate Decay Heat Removal System } \\ \text { AOT } & \text { Allowed Outage Time } \\ \text { ATWS } & \text { Anticipated Transient Without Scram } \\ \text { BWR } & \text { Boiling Water Reactor } \\ \text { CDF } & \text { Core Damage Frequency } \\ \text { CRD } & \text { Control Rod Drive } \\ \text { ECCS } & \text { Emergency Core Cooling System } \\ \text { EDG } & \text { Emergency Diesel Generator } \\ \text { ESF } & \text { Engineered Safety Features } \\ \text { GGNS } & \text { Grand Gulf Nuclear Station } \\ \text { HPCS } & \text { High Pressure Core Spray } \\ \text { INEL } & \text { Idaho National Engineering Laboratory } \\ \text { IPE } & \text { Individual Plant Examination } \\ \text { IRRAS } & \text { Integrated Reliability and Risk Analysis System } \\ \text { ISSP } & \text { Initial Status of Suppression Pool } \\ \text { LCOs } & \text { Limiting Conditions of Operation } \\ \text { LOSP } & \text { Loss of Offsite Power } \\ \text { LPCI } & \text { Low Pressure Coolant Injection } \\ \text { LPCS } & \text { Low Pressure Core Spray } \\ \text { LP\&SD } & \text { Low Pressure and Shutdown } \\ \text { LWR } & \text { Light Water Reactor } \\ \text { MOV } & \text { Motor Operated Valve } \\ \text { NRC } & \text { Nuclear Regulatory Commission } \\ \text { NRR } & \text { Nuclear Regulatory Research } \\ \text { OC } & \text { Operating Condition } \\ \text { OTR } & \text { Outage Time at Refueling } \\ \text { POS } & \text { Plant Operational State } \\ \text { PRA } & \text { Probabilistic Risk Assessment } \\ \text { RCIC } & \text { Reactor Core Isolation Cooling } \\ \text { RHR } & \text { Residual Heat Removal } \\ \text { RWCU } & \text { Reactor Water Clean-up } \\ \text { SDC } & \text { Shutdown Cooling } \\ \text { SNL } & \text { Sandia National Laboratories } \\ \text { SSW } & \text { Standby Service Water } \\ \text { STI } & \text { Surveillance Test Interval } \\ \text { T1 } & \text { LOSP Transient } \\ \text { TAC2 } & \text { Toss of Division II 4160 AC Bus } \\ \text { TS } & \end{array}$




\section{Nomenclature}

\begin{tabular}{ll}
$A_{I C D F}$ & Annual increase in core damage frequency \\
$C_{0}$ & Total core damage frequency with no systems out for maintenance \\
$C_{1}$ & Total core damage frequency when the system is down for maintenance \\
$I_{C D F}$ & Increase in conditional core damage frequency \\
$I_{C D P}$ & Increase in core damage probability over the AOT period \\
$\lambda$ & Standby failure rate of a component \\
$\varpi$ & Maintenance frequency \\
$R_{0}$ & Core damage frequency when component is available \\
$R_{1}$ & Core damage frequency when component is unavailable \\
$R_{B}$ & Risk benefit of the test \\
$R_{D}$ & Incremental risk penalty due to test downtime \\
$R_{N}$ & Risk penalty due to test downtime \\
$T$ & Surveillance test interval \\
$\tau$ & Time between refueling outages \\
$T_{n}$ & Time spent in POS $n$ \\
$T_{P}$ & Time spent at power \\
$T_{R}$ & Timntime \\
\hline
\end{tabular}




\section{Acknowledgements}

The authors wish to thank Mr. Carl Johnson, the NRC program manager, for his invaluable contribution, support and insightful comments during the program. We also wish to acknowledge George Lee, Gary Smith, and Riley Collins of
Grand Gulf Nuclear Station (GGNS), Donnie Whitehead of Sandia National Laboratories and P. K. Samanta of Brookhaven National Laboratory (BNL) for their support and technical contributions. 



\section{Executive Summary}

Recent operational experiences indicate that low power and shutdown accident risks may be important. Consequently, as a part of a Boiling Water Reactor (BWR) Low Power and Shutdown Accident Frequencies Program, the Nuclear Regulatory Commission has undertaken steps towards understanding the risk involved in the operation of Light Water Reactors during low power (less than $15 \%$ power) and shutdown. As an addendum to the program, Sandia National Laboratories has been enlisted to evaluate the risk impact of the Limiting Conditions of Operation in the current Technical Specifications (TSs). This analysis is based on the TSs for Grand Gulf Nuclear Station.

The primary objective of this part of the program is to utilize the models developed in the BWR Low Power and Shutdown Accident Frequencies Program (Phases 1 and 2) to develop and implement methods for evaluating the risk impact of Limiting Conditions of Operation at low power and shutdown. These Limiting Conditions for Operation include Allowed Outage Times (AOTs), i.e., the time allotted for system maintenance outages. The study. aisoanalyzed the risk impact of Surveillance Test Intervals (STIs) during shutdown, i.e., the interval allowed between tests.

The first step in the program was to develop the risk measures based on core damage frequency (CDF) to be used as evaluation criteria for test and maintenance activities at power and at shutdown. The risk measures developed in this step for maintenance on single and multiple systems include:

(1) the Increase in Conditional Core Damage Frequency (i.e., the increase in core damage frequency (CDF) above the baseline (the CDF with all systems available) given a system(s) is unavailable due to maintenance);

(2) the Increase in the Core Damage Probability over the AOT period (i.e., the product of the Increase in Conditional Core Damage Frequency and the AOT);

(3) the Annual Increase in Core Damage Frequency which is the expected increase in the frequency of core damage over a year (i.e., the product of the Increase in the Core Damage Probability and the frequency of the maintenance outage); and

(4) the Outage Time at Refueling which is a postulated outage time at refueling that results in a core damage probability during refueling equivalent to the that given by the AOT at power.
For surveillance tests, the risk measures used include:

(1) the Risk Benefit which is a measure of the decrease in CDF obtained as a result of the detection of failures or degraded conditions that occur during the standby period of a component; and

(2) the Risk Penalty Due to Test Downtime which is a measure of the increase in CDF due to equipment unavailability during a test.

In the second step, eight Plant Operational States (POSs) based on the probabilistic models developed for Phases 1 and 2 of the BWR Low Power and Shutdown Accident Frequencies Program and modifications of the full power NUREG/CR-4550 study were developed to simulate both power operations and shutdown conditions. The POSs are a reclassification of the five modes of operation defined in the TSs and are comprised of POS 0 ( full power operation), POS 1 (low power operation, i.e., less than 15\% thermal power), POS 2 (hot shutdown from rated pressure down to 500 psig), POS 3 (hot shutdown from 500 psig down to about 100 psig), POS 4 (hot shutdown with shutdown cooling operating down to $0 \mathrm{psig}$ ), POS 5 (cold shutdown with temperatures $\leq 200^{\circ} \mathrm{F}$ ), POS 6 (refueling (low) i.e., refueling with the vessel head off and water level raised to the steam lines), and POS 7 (refueling (high) i.e., refueling with the vessel head off and the upper pool filled).

Even though separate models were used to represent the eight POSs, the models were done in sufficient detail to allow for comparisons among the POSs. However, some improvements on the models such as those listed in Section 6.2 of this report would increase the confidence in which comparisons can be made across POSs.

In the third and final step, the risk measures were evaluated using the developed probabilistic models to determine the risk impact of test and maintenance activities (i.e., the risk impact of AOTs and STIs at power and at shutdown).

The following is a summary of the most important results and insights gained from the analysis. The suggested guidelines for enhancing the TS action statements are based solely on the results of this analysis and as such, do not include other factors that could impact the viability of these suggestions.

In order to compare the relative importance of equipment outages, core damage probabilities are categorized, relative to each other, as: 
Executive Summary

$\begin{array}{ll}\text { large } & >1 \mathrm{E}-4 \\ \text { medium } & 1 \mathrm{E}-6 \text { to } 1 \mathrm{E}-4 \\ \text { small } & 1 \mathrm{E}-8 \text { to } 1 \mathrm{E}-6 \\ \text { negligible } & <1 \mathrm{E}-8 .\end{array}$

Similarly, core damage frequencies are categorized, relative to each other, as:

$\begin{array}{ll}\text { high } & >1 \mathrm{E}-4 / \mathrm{yr} \\ \text { medium } & 1 \mathrm{E}-6 / \mathrm{yr} \text { to } 1 \mathrm{E}-4 / \mathrm{yr} \\ \text { low } & 1 \mathrm{E}-8 / \mathrm{yr} \text { to } 1 \mathrm{E}-6 / \mathrm{yr} \\ \text { negligible } & <1 \mathrm{E}-8 / \mathrm{yr} .\end{array}$

\section{Single and Multiple Train Maintenance on the Emergency Diesel Generators (EDGs)}

Comparisons between the baseline CDF and the CDF when an Emergency Diesel Generator (EDG) is unavailable due to maintenance are shown in Figures 1 and 2. (Note that the time axis in Figure 2 is an estimate of the number of days in an operating cycle).

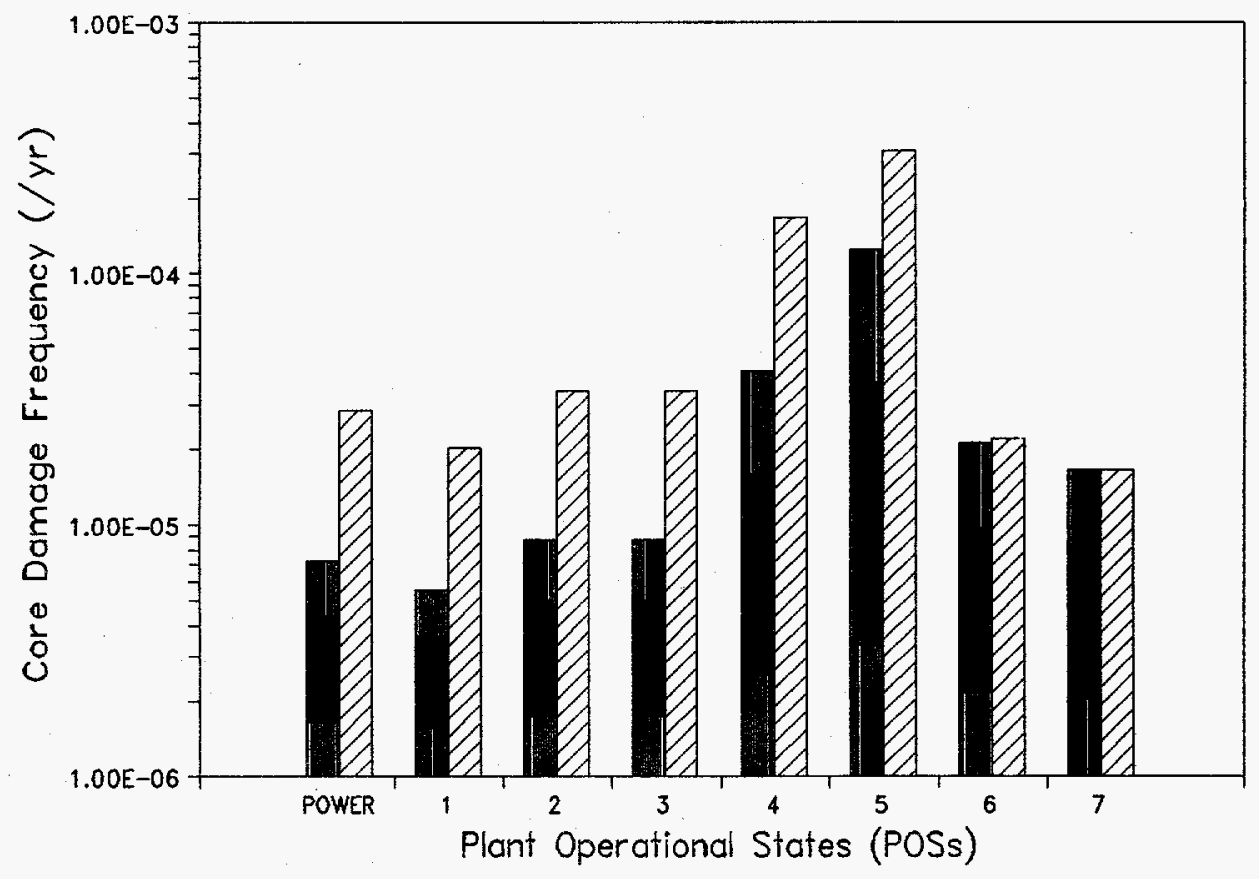

No Maintenance

QZZ EDG in Maintenance

Figure 1. Change in Core Damage Frequency for Taking an EDG Out for Maintenance 


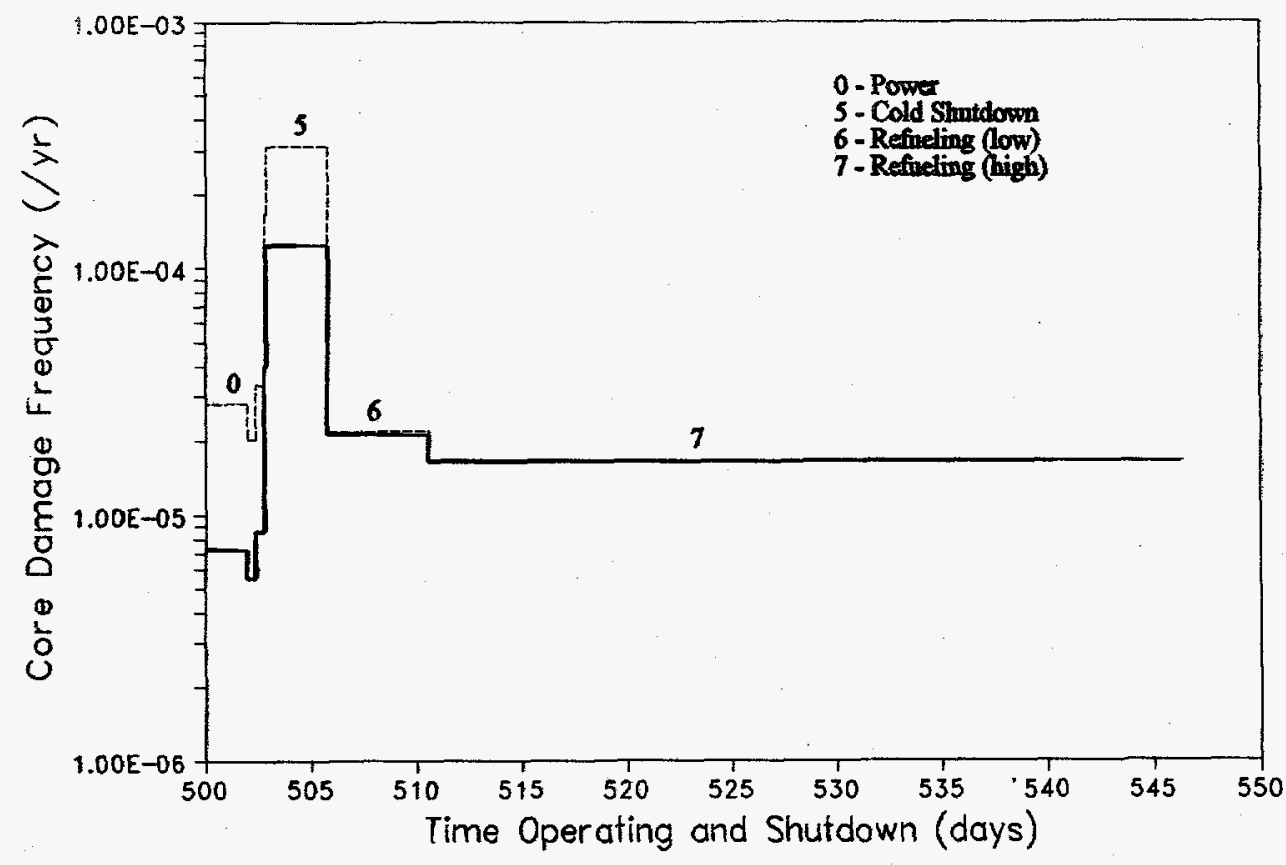

No Minintenance EDG in Maintenance

Figure 2. Change in Core Damage Frequency for Taking a Single EDG Out for Maintenance During the Different POSs in an Operating Cycle

The Increase in Conditional CDF for taking a single EDG out of service during the different POSs in an operating cycle is shown in Figure 3. The figure indicates that single EDG maintenance outages result in a medium to high Increase in Conditional CDF during low power and the first few days of hot and cold shutdown (POSs 1, 2, 3, 4 and 5) which is comparable to that at power (e.g., 4.12E-5/yr for EDG II at power). For simultaneous maintenance on multiple EDGs, i.e., taking Division I and II Emergency Diesel generator (EDG I and EDG II) out of service at the same time, the Increase in Conditional CDF increases to a high level at low power and the first few days of hot and cold shutdown which is also comparable to that at power. However, in both cases, the Increase in Conditional CDF is substantially reduced when the plant is at refueling especially in POS 7 where the decay heat is low and water level is high.

The results discussed above provide the following insights regarding the scheduling of EDG maintenance outages:

- Even though the Increase in Conditional CDF for taking a single EDG out of service at power is medium, preventative maintenance scheduled for a few days (e.g., an AOT of 3 days for EDG In) results in only a small increase in the probability of core damage (e.g., 3.38E-7 for EDG II). However, repeated use of the Limiting Conditions of Operation (LCOs) to 
perform preventative maintenance at power significantly increases the Expected Annual Increase in CDF.

- Based on the Increase in Conditional CDF, there appears to be no risk advantage to performing EDG maintenance during low power and the early stages of shutdown as opposed to power operations.

Preventative maintenance of long or uncertain duration (e.g., EDG overhauls) could preferably be scheduled for refueling (POS 7) where the water level is high and the decay heat is relatively low.

\section{Single and Multiple Train Maintenance on Standby Service Water (SSW) System}

The Increase in Conditional CDF for taking a single train of Standby Service Water (SSW) out of service during the different POSs in an operating cycle is shown in Figure 4. The figure indicates that single train SSW maintenance outages result in a medium Increase in Conditional CDF

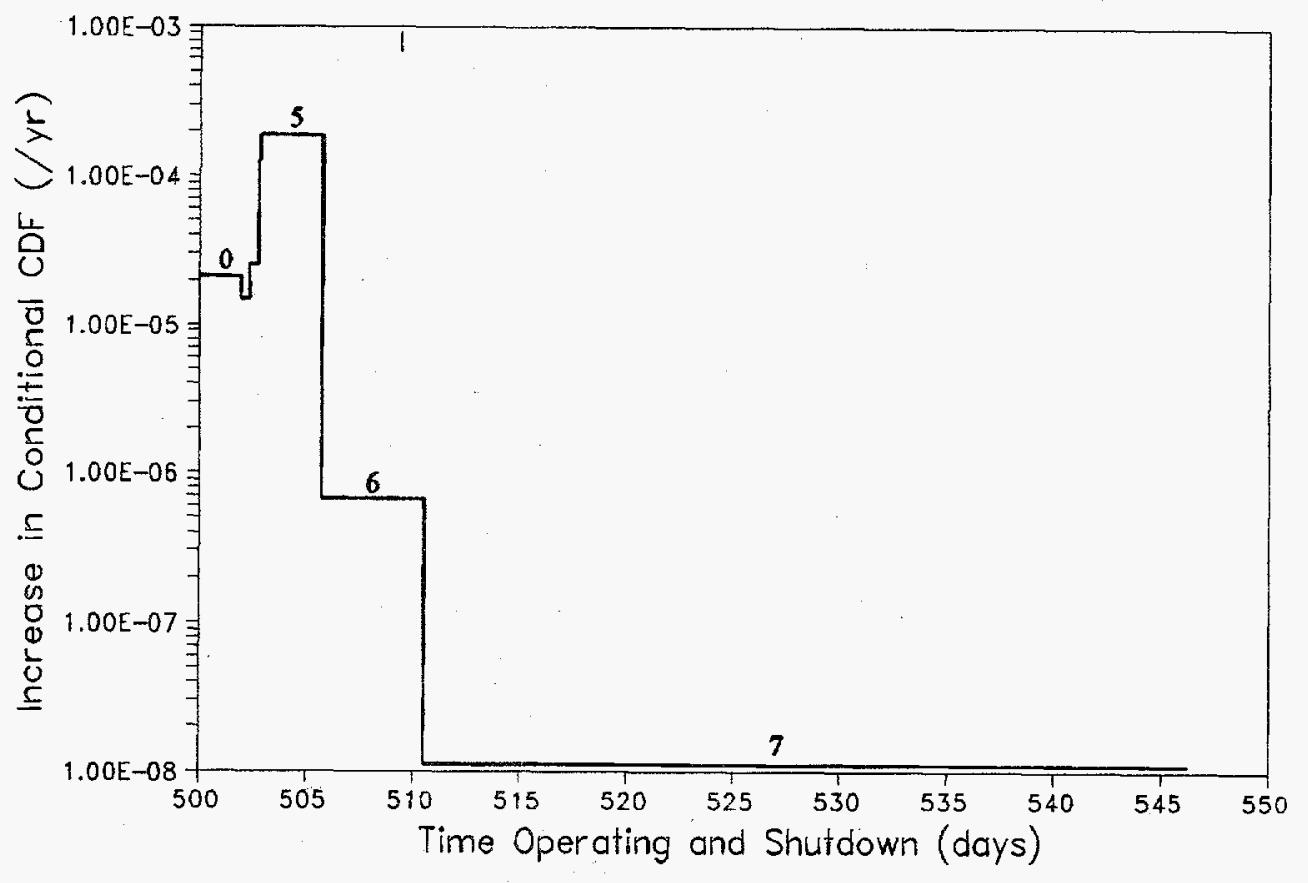

- EDG

Figure 3. Increase in Conditional CDF for Taking a Single EDG Out of Service During the Different POSs in an Operating Cycle 
during low power and the first few days of hot shutdown (POSs 1,2 and 3) which is comparable to that at power (e.g., 2.26E-5/yr for SSW A at power). For POSs 4 and 5, the value increases to the high range and is only slightly reduced, compared to power, with the plant operating in refueling (POS 7).

For multiple maintenance on SSW, the Increase in Conditional $\mathrm{CDF}$ is high during low power and the first few days of hot and cold shutdown (POSs 1, 2, 3, 4 and 5) which is comparable to that at power (e.g., 3.42E-3/yr for SSW Train A and Train C (SSW A and C) at power). However, unlike the EDGs, the Increase in Conditional
CDF when the plant is at refueling, (POS 6 and 7) is not substantially reduced but is also high (e.g., 3.72E-4/yr for SSW A and C in POS 7). The results discussed above provide the following insights regarding the scheduling of SSW maintenance outages:

- Even though the Increase in Conditional CDF for taking a single train of SSW out of service at power is medium, SSW single train maintenance scheduled for only a few days (e.g., 3 days for SSW A) incurs only a small increase in the probability of core damage (e.g. 1.85E-7). However, repeated use of the

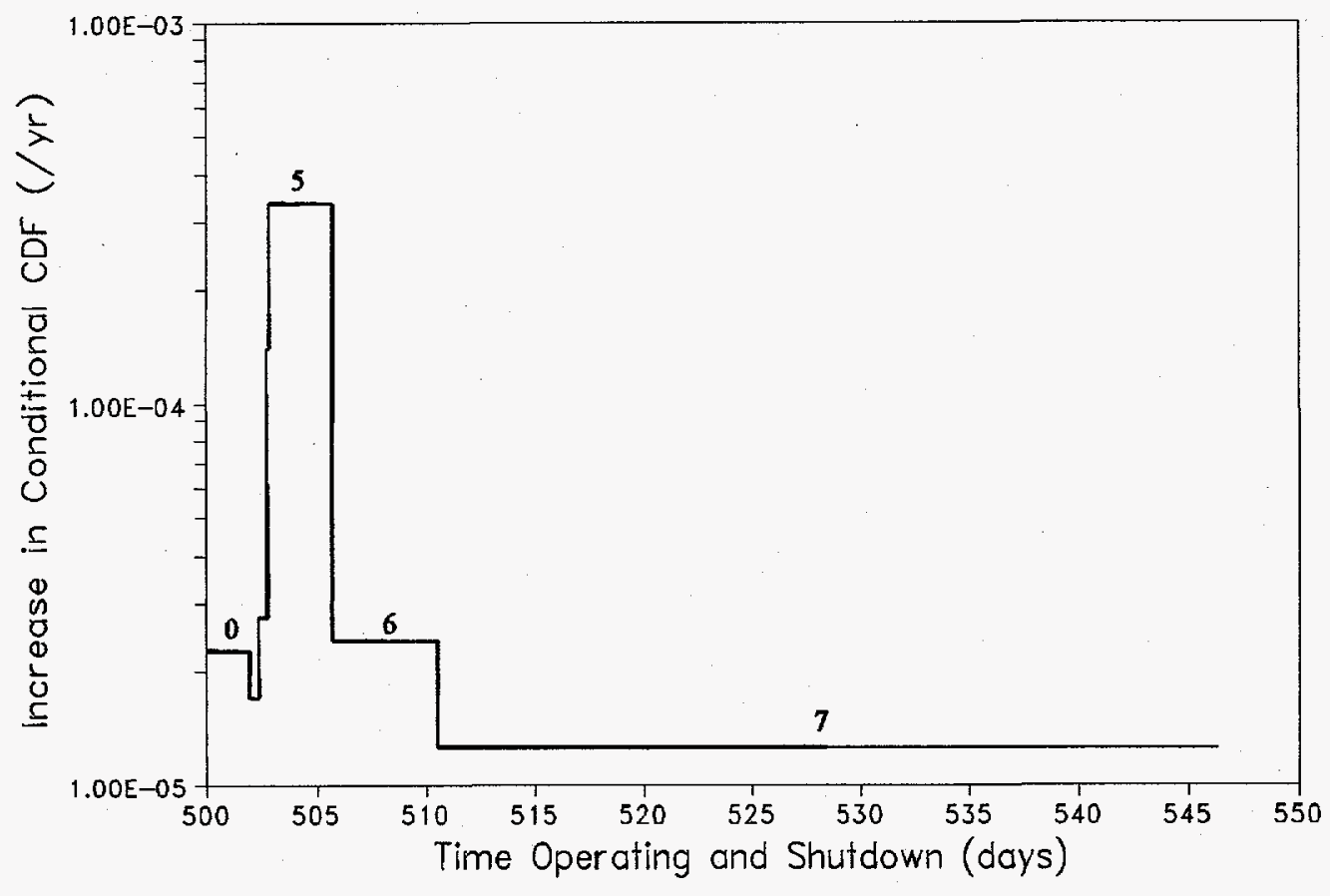

SSW A

Figure 4. Increase in Conditional CDF for Taking a Single Train of SSW Out of Service During the Different POSs in an Operating Cycle 
LCOs to perform preventative maintenance could significantly increase the Expected Annual Increase in CDF.

- Based on the Increase in Conditional CDF, single and multiple train maintenance on SSW should probably not be scheduled for the late phases of hot shutdown and the early phases of cold shutdown, when the Alternate Decay Heat Removal System (ADHRS) is not an alternative for decay heat removal. Scheduled maintenance on SSW trains A and B in these POSs would require the use of emergency procedures.

- The Increase in Conditional CDF for single train maintenance on SSW during refueling is only slightly reduced compared to power. Thus, there is only a slight risk advantage to performing preventative maintenance of long duration at refueling as compared to power. By performing SSW maintenance at power, the maintenance burden at refueling could be reduced.

- Based on the Increase in Conditional CDF, long term multiple train maintenance on SSW should preferably be scheduled at refueling where decay heat is low and ADHRS is available for decay heat removal.

\section{Single and Multiple Maintenance on the Batteries (BATTs)}

The Increase in Conditional CDF for taking a single BATT out of service during the different POSs in an operating cycle is shown in Figure 5. The figure indicates that maintenance on a single Battery results in a

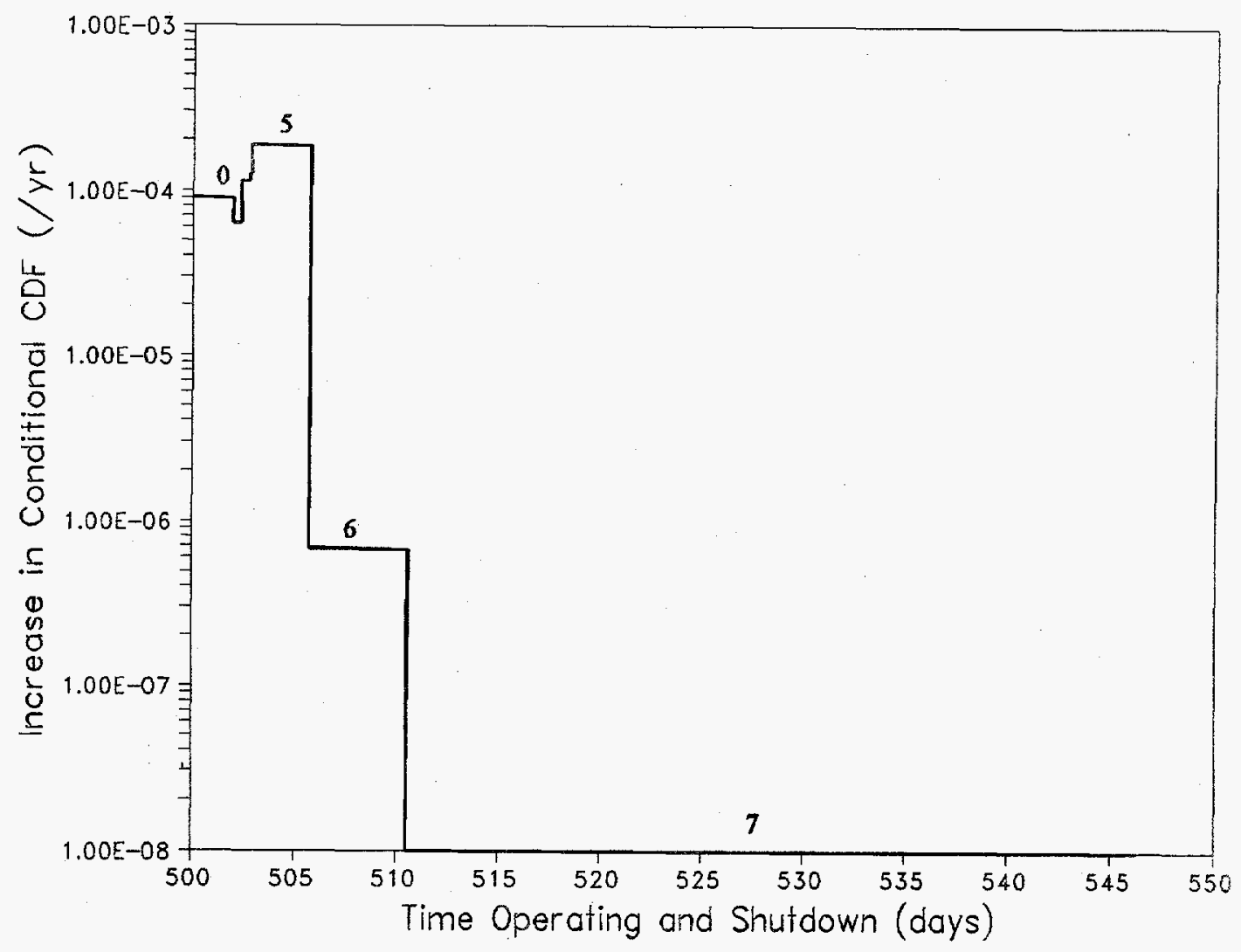

Figure 5. Increase in Conditional CDF for Taking a Single Battery out of Service During the Different POSs in an Operating Cycle 
medium to high Increase in Conditional CDF during low power and the first few days of hot and cold shutdown (POSs 1, 2, 3, 4 and 5) which is comparable to that at power (e.g., 1.40E-4/yr for Division 2 Battery (BATT II) at power). For multiple battery maintenance outages, the results increase to a high level at low power and the first few days of hot and cold shutdown which is also comparable to that at power. However, in both cases, the increase in conditional CDF is substantially reduced with the plant is operating in refueling especially in POS 7 where the decay heat is low and water level is high.

The results discussed above provide the following insights regarding the scheduling of battery maintenance outages:

- $\quad$ Even though the Increase in Conditional CDF for taking a single battery out of service at power is medium, preventative maintenance scheduled for a few hours (e.g., an AOT of 2 hours for BATT II) at power results in only a small increase in the probability of core damage (e.g. 3.20E-8). Since the increase in the probability of core damage is minimal, the possibility of extending the AOT given in the current TS for a few more hours could be considered without creating a substantial increase in core damage probability.

- Based on the Increase in Conditional CDF, there appears to be no risk advantage to performing maintenance on multiple or single batteries during low power and the early stages of shutdown. Like the EDGs, preventative maintenance of long or uncertain duration could preferably be scheduled for refueling (POS 7) where the Increase in Conditional CDF due to battery unavailabilities is lowest.

\section{Single and Multiple Emergency Core Cooling System (ECCS)/Residual Heat Removal (RHR) Maintenance}

The Increase in Conditional CDF for taking High Pressure Core Spray (HPCS) and Low Pressure Core Spray (LPCS) out of service during the different POSs in an operating cycle are shown in Figures 6 and 7, respectively. The figures indicate that maintenance on HPCS and LPCS result in medium increases in the Conditional CDF during the first few days of low power and hot shutdown (POSs 1, 2, and 3) which is comparable to that at power. These values increase in POSs 4 and 5. For HPCS, the increase in conditional CDF compared to power is only marginally reduced in POSs 6 and 7 . For LPCS, the increase in conditional CDF compared to power for POSs 6 and 7 is also marginally reduced but is a slightly larger reduction than that for HPCS.

The Increase in Conditional CDF for taking a Low Pressure Coolant Insertion (LPCI) out of service during the different POSs in an operating cycle is shown in Figure 8. The figure indicates that for maintenance on a single train of LPCI, the increase in Conditional CDF is low during low power and the first few days of hot shutdown (POSs 1, 2 and 3) which is comparable to that at power. However, it increases to the medium range with the plant operating in POSs 4 through 7 . The same trend occurs for multiple train maintenance on LPCI.

The results discussed above provide the following insights regarding the scheduling of ECCS maintenance outages:

- Even though the Increase in Conditional CDF for HPCS and LPCS are in the medium range and in the low range for LPCI, scheduling preventative maintenance of up to 14 days for HPCS and 7 days for LPCS and a single train of LPCI at power result in only a small increase in the probability of core damage. Thus, the AOTs for LPCS and LPCI could possibly be considered for an extension to 14 days without creating a substantial increase in the core damage probability at the plant. This extension could allow more time for proper repair of LPCS and LPCI and also create a uniform ECCS AOT for HPCS, LPCS, and LPCI.

- When adhering to the current AOT of 14 days for HPCS, excessive use of the LCOs to perform preventative maintenance at power could significantly increase the Expected Annual Increase in CDF.

- Based on the Increase in Conditional CDF, there appears to be no risk advantage in performing maintenance on LPCS, LPCI, or HPCS during the early stages of shutdown. Multiple train maintenance on LPCI should probably not be scheduled for the late phases of hot shutdown and the eariy phases of cold shutdown, when Alternate Decay Heat Removal System (ADHRS) is not an alternative for decay heat removal. Scheduled maintenance on LPCI trains A and $B$ in these POSs would require the use of emergency procedures. 
Executive Summary

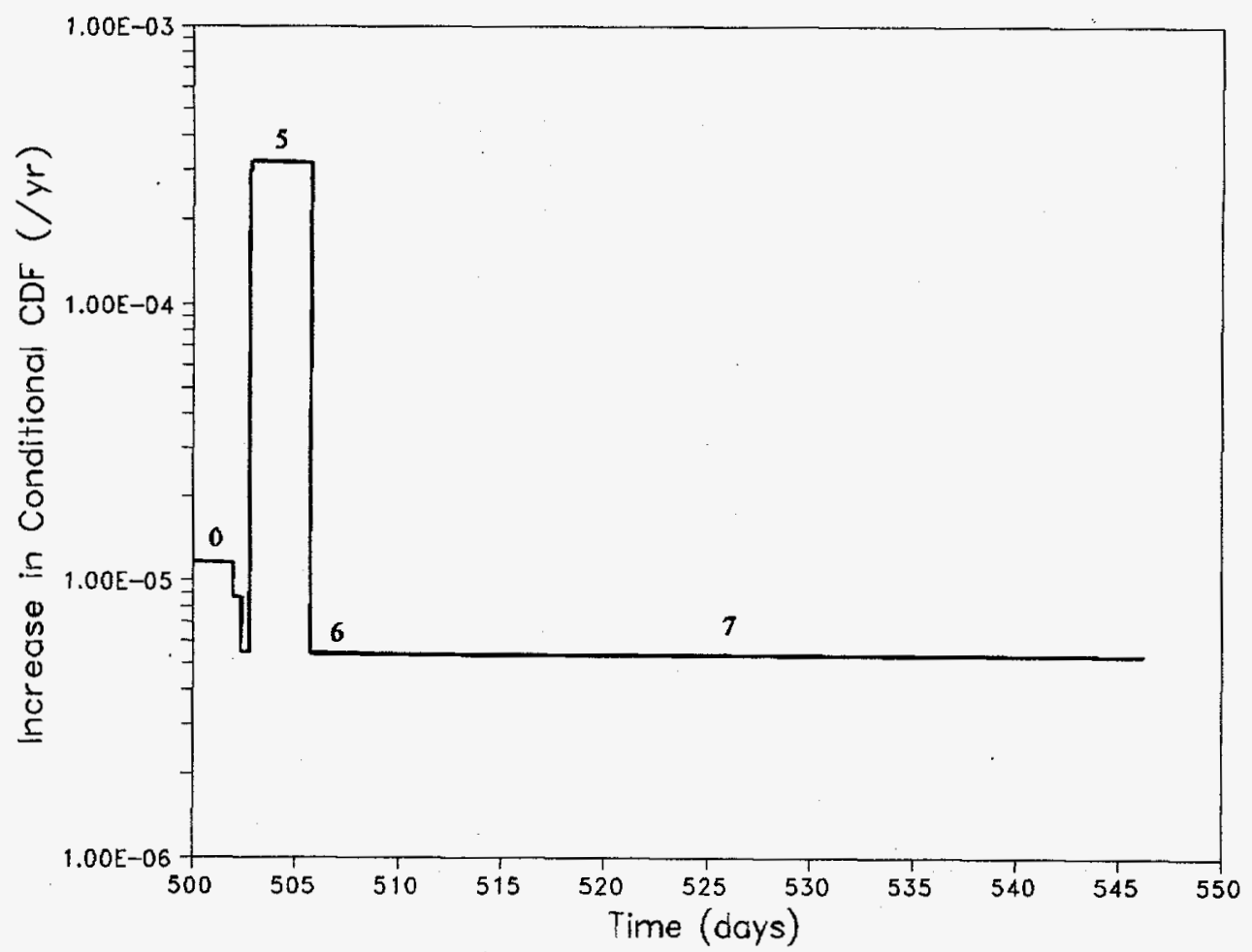

Figure 6. Increase in Conditional CDF for Taking HPCS Out of Service During the Different POSs in an Operating Cycle. 


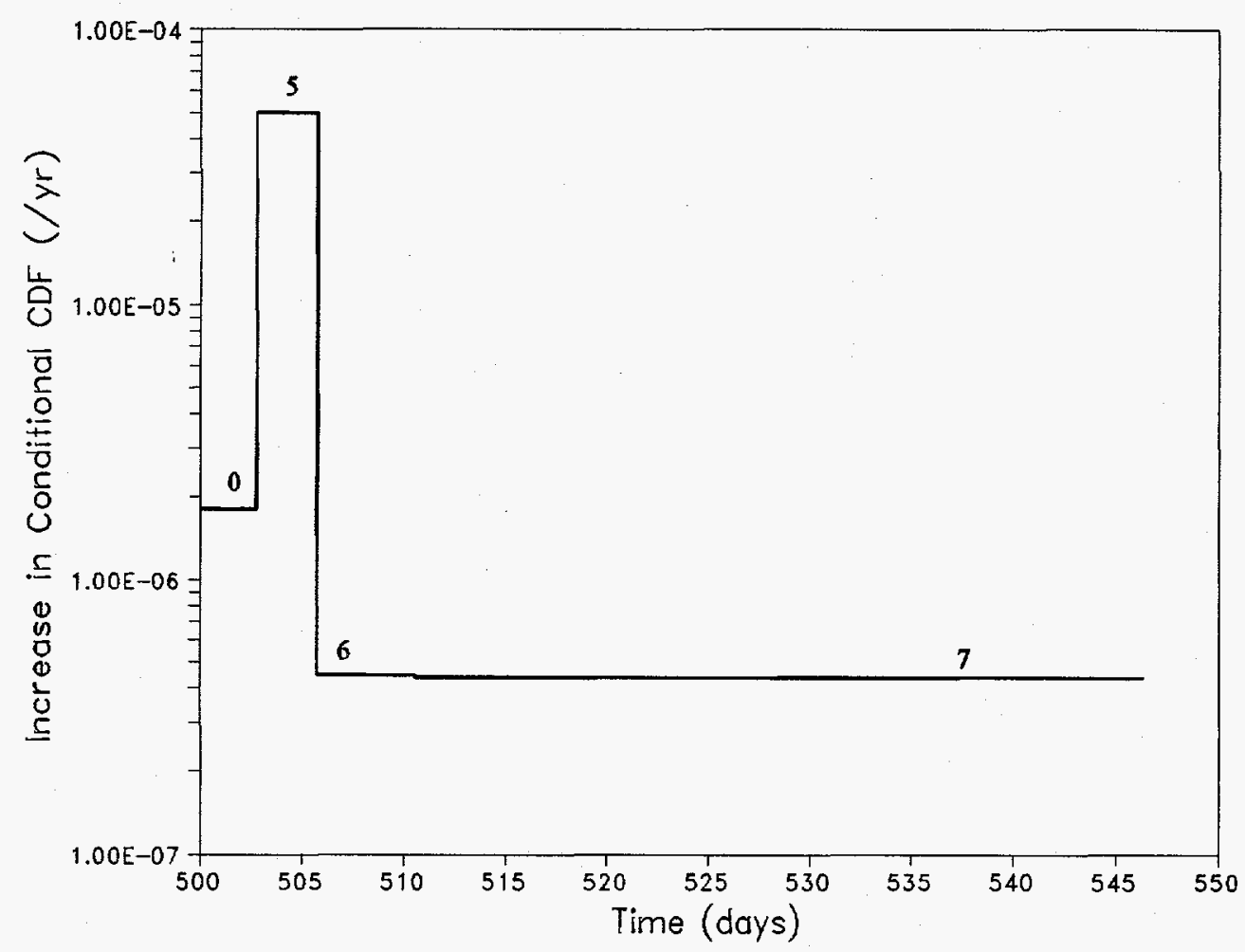

Figure 7. Increase in Conditional CDF for Taking LPCS Out of Service During the Different POSs in an Operating Cycle 
Executive Summary

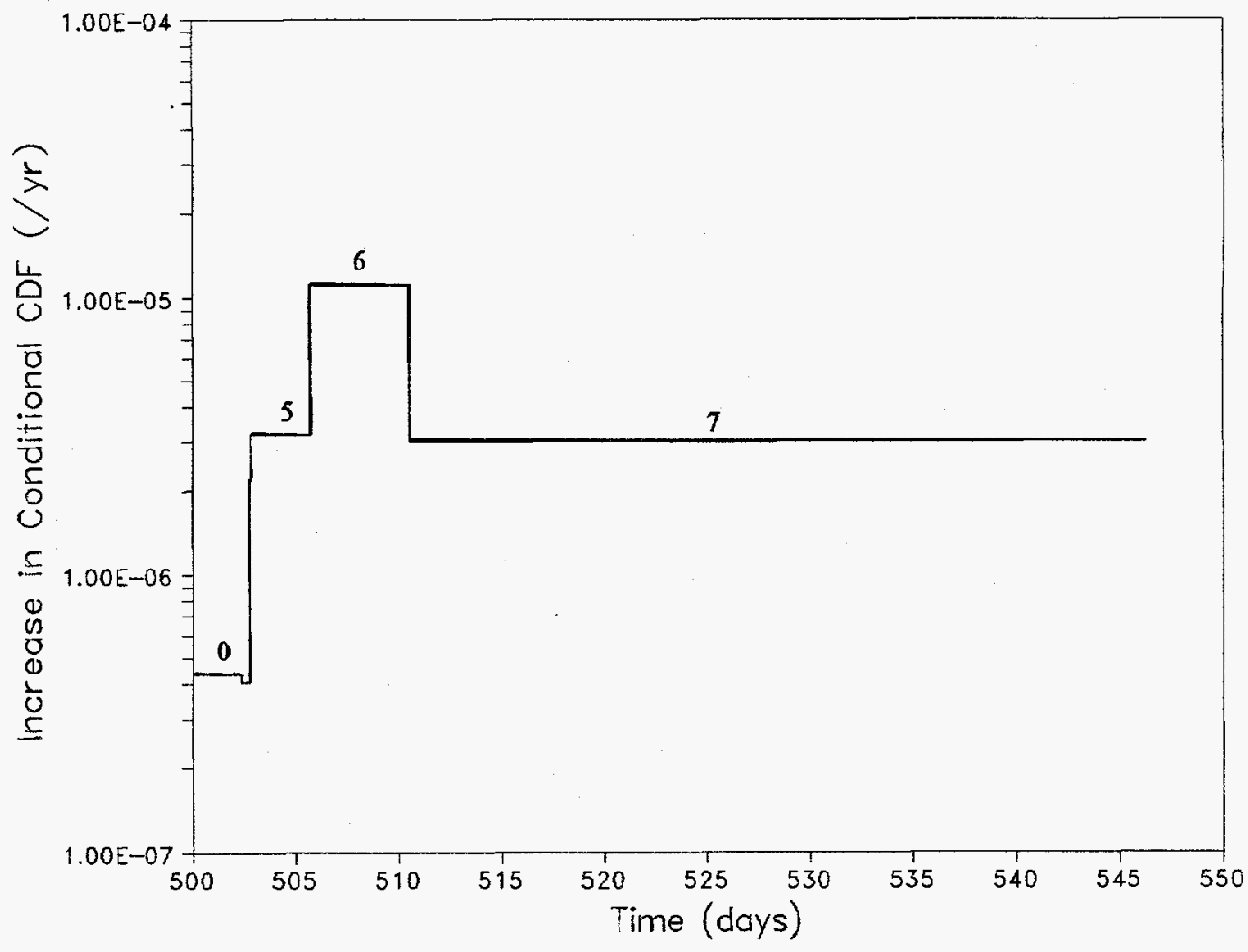

Figure 8. Increase in Conditional CDF for Taking LPCI A Out of Service During the Different POSs in an Operating Cycle

- There is only a small difference in the Increase in the Conditional CDF when performing HPCS maintenance at refueling compared to power. Thus, there appears to be only a slight advantage to performing preventative maintenance of longer than 14 days (e.g., HPCS overhauls) at refueling as compared to power. For LPCS, there appears to be a slightly greater advantage to performing maintenance of longer than 7 days at refueling as compared to power. In comparison to power, there appears to be no risk advantage to performing maintenance on single or multiple trains of LPCI at refueling. Thus scheduling maintenance on HPCS, LPCS, and LPCI at power as opposed to refueling could possibly reduce the maintenance burden at refueling. 


\section{Maintenance on the Suppression Pool or all Safety Relief Valves (SRVs)}

Drainage of the suppression pool in POSs 4 through 7 for maintenance or simultaneous maintenance on all Safety Relief Valves (SRVs) in POSs 4 and 5 result in high Increases in Conditional CDF (e.g., 1.62E-2 for SRVs in POS 4). Therefore,

- Drainage of the suppression pool for maintenance or simultaneous maintenance on all SRVs are high risk activities that should preferably be scheduled for refueling (POS 7) where the water level is high and the Increase in Conditional CDF is lowest.

\section{Surveillance Testing at Power and Shutdown}

- There is a medium Risk Benefit associated with the monthly start test on the EDGs (e.g., 1.07E-6/yr for EDG I) and the quarterly flow tests for HPCS and the Reactor Core Isolation Cooling (RCIC) system. Therefore, these tests are effective in controlling the potential risk at power and do not appear to be candidates for STI extensions.

- Since there is a low Risk Benefit associated with ECCS quarterly stroke tests and the weekly battery surveillance at power, extensions of their STIs could probably be considered with only a small increase in risk. Such an extension could reduce the operational burden at the plant resulting in more time for accomplishing more risk significant test activities.

At power, the Risk Benefit of quarterly flow testing the low pressure Emergency Core Cooling System (ECCS) systems (i.e., LPCS and LPCI train A) is low and is less than that for the high pressure coolant systems. For these tests, extensions of the STIs could possibly be considered which could possibly reduce the operational burden at the plant by allotting more time to accomplish more significant risk control activities.
- There is a low Risk Benefit associated with the quarterly SSW flow test at power. This is due to the fact that any failures in the SSW can usually be detected during the frequent testing of other safety systems such as the EDGs which depend on SSW. Thus, the SSW flow test appears to be a possible candidate for STI extension.

- Generally, there is only a low to negligible Risk Penalty due to Test Downtime associated with tests performed during shutdown and refueling. These tests include the ECCS flow test, the ECCS 18 month logic system functional test, the EDG $24 \mathrm{hr}$ run/load test, the EDG 18 month functional test simulating a loss of power (LOP), the ECCS 18 month valve stroke test and the 18 month battery emergency load test. Therefore, these tests could probably be performed in POS 5,6 , or 7 , without incurring significant risk.

- At shutdown and at refueling, the Risk Benefit of performing the EDG $24 \mathrm{hr}$ run/load test is significant (e.g. 1.57E-5/yr for EDG I). The Risk Benefit is medium for the ECCS logic system functional tests (e.g., 1.24E-6/yr for LPCS). The Risk Benefit during shutdown is low for the ECCS flow test, the EDG 18 month functional test simulating a loss of power (LOP), the ECCS 18 month valve stroke test, and the 18 month battery emergency load test. However, these surveillances, which can only be performed during plant shutdown, are necessary to assure system availability during subsequent power operations.

As stated previously, these results and insights were derived solely from a CDF perspective. Additional research might be necessary before implementing any of the items proposed above. In addition, since GGNS has some uncommon design safety features such as ADHRS, extrapolation of these results to other BWRs without additional analyses is not recommended. 


\subsection{Introduction}

\subsection{Background}

Probabilistic risk assessments (PRAs) of severe accidents in nuclear power plants are traditionally done for full power operations. However, recent operational experiences indicate that low power and shutdown accident risks may be significant and should not be ignored. Consequently, the Nuclear Regulatory Commission (NRC) has undertaken a two-phased BWR Low Power and Shutdown Accident Frequencies Program in order to understand the risk involved in the operation of Light Water Reactors (LWRs) during low power (less than $15 \%$ power) and shutdown. As an addendum to the program, Sandia National Laboratories (SNL) has been enlisted to perform risk evaluations to determine if the Limiting Conditions of Operation (LCOs) in the current Technical Specifications (TSs) provide adequate risk controls at shutdown.

\subsection{Objective}

The primary objective of this extension of the program is to utilize the models developed in the Boiling Water Reactor (BWR) Low Power and Shutdown Accident Frequencies Program (LP\&SD) for Grand Gulf Nuclear Station (GGNS), Phase 1 [Whitehead et al., 1991] and Phase 2 [Whitehead et al., 1992], and the full power NUREG/CR-4550 [Drouin et al., 1989] to develop and implement methods for evaluating the risk impact of LCOs at low power and shutdown. These LCOs are comprised of the Allowed Outage Times (AOTs), i.e., the time allotted for system maintenance outages; and the Surveillance Test Intervals (STIs), i.e., the interval allowed between tests. The LCOs provide the basis for evaluating the risk impact of the TSs.

\subsection{Approach}

The first step in the program was to develop the risk measures, based on core damage frequency (CDF), to be used as evaluation criteria for test and maintenance activities at power and at shutdown.

In the second step, eight Plant Operational States (POSs) based on the probabilistic models developed for Phases 1 and 2 of the LP\&SD Program and the full power NUREG/CR-4550 study were developed to simulate both power operations and shutdown conditions. The POSs are a reclassification of the five modes of operation called operating conditions (OCs): power (OC 1), startup (OC 2), hot shutdown (OC 3), cold shutdown (OC 4), and refueling (OC 5). The POSs are defined as follows: (i) POS 0 consisting of: full power operation at rated pressure (1000 psig) and thermal power greater than $15 \%$.

(ii) POS 1 consisting of: low power operation with pressure at rated conditions (about $1000 \mathrm{psig}$ ) and thermal power no greater than $15 \%$.

(iii) POS 2 consisting of: hot shutdown from rated pressure down to $500 \mathrm{psig}$.

(iv) POS 3 consisting of: hot shutdown from 500 psig down to where Residual Heat Removal (RHR)/ Shutdown Cooling (SDC) is initiated (about 100 psig).

(v) POS 4 consisting of: hot shutdown with RHR/SDC operating down to $0 \mathrm{psig}$.

(vi) POS 5 consisting of: cold shutdown $(T \leq 200 \mathrm{~F})$ until the vessel head is off and water level is raised to the steam lines (refueling).

(vii) POS 6 consisting of: refueling with the head off and water level raised to the steam lines.

(viii) POS 7 consisting of: refueling with the head off, the upper pool filled, and the refueling transfer tube open.

In the third and final step, the risk measures were evaluated using the developed probabilistic models to determine the risk impact of test and maintenance activities, i.e., the risk impact of AOTs and STIs at power and at shutdown.

\subsection{Report Organization}

The report is organized as follows: Section 2 presents the basic concepts of the methodology used to evaluate the risk impact of the AOTs and STIs at power and at shutdown. Sections 3 and 4 present results and reveal insights of the risk impact of single and multiple system maintenance and surveillance test activities, respectively. Section 5 provides the general conclusions of the study, and Section 6 describes the limitations of the analysis and provides recommendations for future research. Appendix A presents the system maintenance configurations and their corresponding CDF estimates used to evaluate the risk impact of maintenance activities. Appendix B presents the component surveillance test configurations and the raw data used to evaluate the risk impact of surveillance tests.

Appendix $\mathrm{C}$ lists the numerical results of the risk impact of 
Introduction

single and multiple maintenance activities. Appendix D lists the numerical results of the risk impact of surveillance test activities, and Appendix E presents descriptions of systems used in this analysis. 


\subsection{Methodology}

This section of the report describes the methodology used to study the risk impact of single and multiple system outages due to test and maintenance activities. This methodology is based on the models, assumptions, and results of the Grand Gulf LP\&SD (Phases 1 and 2), NUREG/CR-4550, and the risk measures based on changes in CDFs due to test and maintenance activities.

The first step in the methodology is to define the risk based measures to be used as evaluation criteria for test and maintenance activities at power and at shutdown. The second step is to develop PRA models based on the results and assumptions from the Grand Gulf LP\&SD (Phases 1 and 2) and the full power NUREG/CR-4550 PRA to simulate both power operations and shutdown conditions. The third and final step is to use the developed risk measures to evaluate the PRA models and determine the risk impact of test and maintenance activities at power and at shutdown.

\subsection{Definitions of Risk Based Measures}

This section describes the methodology utilized to define the risk based measures which are used as evaluation criteria for test and maintenance activities at power and at shutdown.

\subsubsection{Definitions of Risk Measures for Evaluating the Impact of Maintenance Activities}

The principal risk measure used to analyze the impact of maintenance activities is the Increase in Conditional Core Damage Frequency, $\mathrm{I}_{\mathrm{CDF}}$. The $\mathrm{I}_{\mathrm{CDF}}$ is defined as

$$
I_{\alpha p}=C_{1}-C_{0} .
$$

where $C_{1}$ is the total CDF when the system(s) of a particular configuration is down for maintenance and $C_{0}$ is the baseline CDF (see Appendix A) assuming systems are not down for maintenance [Staple et al., 1993]. $C_{1}$ is determined by setting the component's unavailability to one, while $C_{0}$ is calculated by setting the maintenance unavailability of the systems to zero in the appropriate PRA model described in Section 2.2. All accident sequences affected during the evaluation of $C_{0}$ and $C_{1}$ were requantified to generate new cut sets. Following the generation of the cut sets, appropriate recovery terms were applied. By regenerating the cut sets, problems with truncation errors were eliminated.

\subsubsection{Risk Measures of Allowed Outage Times for Single Systems}

In assessing the risk impact of maintenance outages the AOT is also important. The risk impact of the AOT is termed the Incremental Increase in Core Damage Probability $\left(\mathrm{I}_{\mathrm{cpp}}\right)$. The $\mathrm{I}_{\mathrm{cDp}}$ is defined as the incremental increase in the probability of core damage over the AOT period and is determined with the assumption that the downed system is out for the total AOT [Samanta et al., 1988].

Mathematically, the $I_{c D P}$ can be written as

$$
I_{C D P}=\left(C_{1}-C_{0}\right) \cdot A O T .
$$

For cases where the AOT was unlimited according to the TSs, the fraction of time spent in the POS was used as the AOT's upper limit. These fractions are listed in Table 1.

The Annual Increase in Core Damage Frequency $\left(\mathrm{A}_{\mathrm{ICDF}}\right)$ is the expected increase in core damage frequency over a year and is equivalent to the product of the $\mathrm{I}_{\mathrm{CDP}}$ and the maintenance frequency of the component(s), $\omega$. That is

$$
A_{10 O P}=I_{C O P} \cdot \omega .
$$

Generic maintenance frequencies for various components are listed in Table 2. During power operations, $\omega$ can be varied; however, during shutdown, preventative or corrective maintenance is normally scheduled once, based on degradations observed at power. Therefore, $\omega$ and hence the $A_{I C D F}$ are not of concern at shutdown.

The final measure used in the analysis is the Outage Time at Refueling (OTR). This is the relative, non-binding, time frame that gives a core damage probability at refueling comparable to that at power. The OTR can be used as a guide for scheduling preventative maintenance at shutdown as opposed to power which could improve the correspondence between the TS requirements for power and shutdown.

\subsubsection{Risk Measures of Allowed Outage Times for Multiple Systems}

During low power and shutdown, plant personnel may perform multiple maintenance and testing activities. These 
multiple outage configurations can result in a significant increase in plant risk as compared to single system outages. By identifying high risk multiple system outages, adverse configurations can be controlled, and LCOs can be defined to optimize outage durations and confine outage scheduling to those POSs where the risk implications are minimal. The multiple component outages investigated in this report include combinations of components that were identified from the dominant cut sets in the PRA model, the GGNS refueling outage schedule, and the specified configurations in the TSs.

To address the risk impact of multiple system outages, the $I_{C D P}$ and the $A_{I C D F}$, when an AOT is not specified, are calculated using the shortest AOT of any single system within the configuration. This is based on the fact that once the shortest AOT in the configuration is exceeded, the TSs direct the plant to shutdown or undertake some other action.

\subsubsection{Definitions of Risk Measures for Evaluating the Impact of Surveillance Test Activities}

Over the lifetime of a system, failures may occur during the standby period, upon demand for operation, or during operation following a successful demand. Failures occurring during the standby period remain undetected until they are revealed by some testing procedure. These failures are called standby related failures. Surveillance testing is a primary tool for assessing the reliability of equipment that is on standby. One measure of the effectiveness of such testing is termed the risk impact of surveillance testing. This measure consists of factors which can be categorized into two competing categories, the Risk Benefit of the test and the Risk Penalty due to the test [Samanta et al., 1992].

The Risk Benefit of the test, $R_{B}$, is the reduction in CDF obtained as a result of the detection of component failures or degraded conditions that occur during the standby period of a component. Samanta et al., (1988) defined $R_{B}$ as

$$
R_{B}=\frac{1}{2} \lambda \cdot T\left(R_{1}-R_{o}\right)
$$

where $R_{o}$ is the CDF when the component is available, $R_{l}$ is the CDF when the component is unavailable, $\lambda$ is the standby failure rate of the component and is assumed to be a constant, and $T$ is the surveillance test interval, i.e., the time between tests. Generic $\lambda$ for various components are listed in Table 3.

The Risk Penalties due to the test are the adverse effects that occur because

the test itself causes wear on the equipment (testcaused wear),

Table 1. Time Spent in Each POS in Transition to Refueling

\begin{tabular}{|c|c|}
\hline POS & Time Spent (hours) \\
\hline 1 & 8.8 \\
\hline 2 & 5.2 \\
\hline 3 & 5.2 \\
\hline 4 & 1.7 \\
\hline 5 & 70.0 \\
\hline 6 & 113.9 \\
\hline 7 & 368 \\
\hline
\end{tabular}


Table 2. Generic Maintenance Frequencies

\begin{tabular}{|c|c|c|}
\hline Component & $\begin{array}{c}\omega \\
\text { (per year) }\end{array}$ & Source \\
\hline Battery & 0.9 & IPRDS \\
\hline Motor Driven Pumps & 0.9 & ASEP \\
\hline Motor Operated Valve & 0.3 & IPRDS \\
\hline Turbine Driven Pump & 2.6 & ASEP \\
\hline Diesel Generator & 2.6 & NUREG/CR-2989 \\
\hline Diesel driven Pump & 0.9 & ASEP \\
\hline
\end{tabular}

Table 3. Generic Standby Failure Rates

\begin{tabular}{|c|c|c|}
\hline Component & $\begin{array}{c}\lambda \\
\text { (per hour) }\end{array}$ & $\begin{array}{c}\text { Source } \\
\text { Motor Driven Pumps }\end{array}$ \\
\hline $\begin{array}{c}\text { Motor Operated } \\
\text { Valve }\end{array}$ & $8.3 \mathrm{E}-6$ & $\begin{array}{c}\text { NUREG/CR-4550 Vol. } \\
1\end{array}$ \\
\hline $\begin{array}{c}\text { Turbine Driven } \\
\text { Pump }\end{array}$ & $8.3 \mathrm{E}-5$ & $\begin{array}{c}\text { NUREG/CR-4550 Vol. } \\
1\end{array}$ \\
\hline Battery & $1.6 \mathrm{E}-6$ & $\begin{array}{c}\text { NUREG/CR-4550 Vol. } \\
1\end{array}$ \\
\hline $\begin{array}{c}\text { Diesel Generator } \\
1\end{array}$ & $8.3 \mathrm{E}-5$ & $\begin{array}{c}\text { NUREG/CR-4550 Vol. } \\
1\end{array}$ \\
\hline Actuation Logic & $4.4 \mathrm{E}-6$ & $\begin{array}{c}\text { NUREG/CR-4550 Vol. } \\
1\end{array}$ \\
\hline Diesel driven Pump & $8.3 \mathrm{E}-5$ & $\begin{array}{c}\text { NUREG/CR-4550 Vol. } \\
1\end{array}$ \\
\hline
\end{tabular}


- the test causes a transient leading to an unscheduled plant shutdown (test-caused transient),

- $\quad$ equipment is not restored to its proper operable state following the test (human error contribution), or

- the equipment is unavailable due to the test (Risk Penalty due to Test Downtime, $R_{N}$ ).

For tests periodically performed during full power operations, the benefit of each test is primarily due to the assurance that the component tested will perform its intended function when required at power [ Deem et al., Draft 1992]. For example, the Emergency Diesel Generators (EDGs) are start tested monthly to assure their availability given a loss of offsite power (LOSP) at the plant. In this case, Equation 4 is applicable. However, at power, the Risk Penalty due to tests are usually assumed to be either negligible or requiring additional studies. This is due to the facts that test downtime is usually short at power, and in many cases, the equipment is not made inoperable by the test. By considering only the Risk Benefit, the net benefit of the test is bounded. Hence, tests resulting in low benefits will have even lower impacts when Risk Penalties are factored in [Samanta et al., 1988].

Two types of tests are considered at shutdown. The first type (Type 1) are tests performed only during shutdown. The second type (Type 2) are tests performed at shutdown which are also required at power.

For Type 1 tests, $R_{B}$ can be written as

$$
\begin{aligned}
R_{B} & =\frac{1}{2} \lambda \cdot T_{p}\left(R_{1}-R_{0}\right)_{p} \\
& +\sum_{n=1}^{N} \frac{1}{2} \lambda \cdot T_{n}\left(R_{1}-R_{0}\right)_{n}
\end{aligned}
$$

where $N$ is the total number of POSs, $T_{p}$ is the time spent at power, $T_{n}$ is the time spent in POS $n$, excluding POS 0 . $\left(R_{I}-R_{o}\right)_{p}$ and $\left(R_{l}-R_{o}\right)_{n}$ are the increases in CDF evaluated at power and in $\operatorname{POS} n$, respectively.

For Type 2 tests, the risk benefit of the test is POS specific and can be approximated as

$$
R_{B n} \approx \sum_{n=1}^{n} \frac{1}{2} \lambda \cdot T_{n}\left(R_{1}-R_{0}\right)_{n}
$$

It is assumed that tests are performed just before shutdown. ,
During a typical shutdown for refueling, only a few hours are spent in transitioning from power operations (POS 0) to cold shutdown (POS 5). This transition time is small compared to the time spent in POS 5, POS 6, or POS 7 ( See Table 1). That is, $T_{1}, T_{2}, T_{3}$, and $T_{4}<<T_{5}, T_{6}$, and $T_{7}$. As a result, most of the testing of equipment at shutdown occurs in POS 5, POS 6, or POS 7.

Consequently, for this evaluation, the risk impact of testing various equipment at shutdown will be limited to these three POSs. Therefore, the risk benefit of Type 1 tests at shutdown can be approximated as

$$
\begin{aligned}
R_{B} & \approx \frac{1}{2} \lambda \cdot T_{p}\left(R_{1}-R_{o}\right)_{p} \\
& +\sum_{n=5}^{7} \frac{1}{2} \lambda \cdot T_{n}\left(R_{1}-R_{o}\right)_{n}
\end{aligned}
$$

For Type 2 tests the risk benefit can be approximated as

$$
R_{B n}=\sum_{n=5}^{n} \frac{1}{2} \lambda \cdot T_{n}\left(R_{1}-R_{o}\right)_{n}
$$

When a test involves the surveillance of multiple components, Eqs. 7 and 8 can become complicated. In this case, the surveillance test interval, $T_{i}$, the standby failure rate, $\lambda_{i}$, and the increase in core damage frequency, $\left(R_{l i}\right.$ $R_{o i}$ ), for each system being tested, $i$, are used in Eqs. 7 and 8 and summed over $i$.

In both Type 1 and 2 tests, the Risk Penalty at shutdown can be significant. This is due to the fact that for many tests performed at shutdown, the equipment is unavailable for extended periods of time. In these cases, the Incremental Risk Penalty due to Test Downtime, $\mathrm{R}_{\mathrm{D}}$, can be expressed as

$$
R_{D}=\left(R_{1}-R_{0}\right) \tau
$$

where $\tau$, the test downtime, is the total time that the equipment is declared inoperable during the test [Deem, et al., Draft 1992].

When $R_{D}$ is normalized by the time between refueling outages, $T_{R}(\approx 18$ months), it is termed the Risk Penalty due to Test Downtime, $\mathrm{R}_{\mathrm{N}}$, and is given by

$$
R_{N}=\frac{R_{D}}{T_{R}}
$$




\subsection{GGNS Model Development}

This section of the report describes the PRA models developed for the eight POSs defined in Section 1.1. These models provide the framework for evaluating the risk impact of various test and maintenance activities at power and at shutdown.

\subsubsection{PRA Model for Power (POS 0)}

The PRA model used for POS 0 was an enhanced version of the NUREG/CR-4550 internal events analysis for GGNS. The analysis in this study was limited to working with accident sequences found to be dominant contributors to the core damage frequency. The NUREG/CR-4550 core damage frequency is dominated by station blackout scenarios, that is, a Loss of Off Site Power (LOSP) initiating events (T1) followed by failure of the emergency diesel generators to provide onsite power.

The NUREG/CR-4550 analysis did not consider special initiators. Therefore, it was deemed necessary to examine Grand Gulf's Individual Plant Examination (IPE) [Smith et al., 1992] to determine if any other initiators were dominant contributors to core damage. This review revealed that special initiators were indeed significant contributors to the CDF in the IPE, in particular the loss of the Division II 4160 AC bus (initiating event TAC2). The LOSP accident sequences were still the dominant contributors to core damage in the IPE (55\% of total), but the TAC2 initiating event contributed about $19 \%$ of the total IPE core damage frequency.

To better facilitate this study, the NUREG/CR-4550 PRA model was enhanced to include the TAC2 initiating event. Including TAC2 in the existing PRA provides a better PRA model for Grand Gulf at full power and broadens the range of this study to include more systems which are needed in this analysis.

To incorporate the TAC2 initiating event, the sequence logic for the dominant TAC2 accident sequences were developed using the Integrated Reliability and Risk Analysis System (IRRAS) code version of NUREG/CR-4550 for Grand Gulf provided by Idaho National Engineering Laboratory (INEL). These accident sequences were then quantified using the NUREG/CR-4550 fault trees. Following a recovery analysis, where the recovery events used in the IPE were carried over to this analysis, the resulting cut sets were compared with the IPE cut sets for the sequences of interest. As a result, some revisions to the NUREG/CR-4550 fault trees were needed in order to include some of the dominant failure modes identified in the IPE cut sets.
The TAC2 sequences were requantified, and the resulting cut sets were again compared with the IPE cut sets. The dominant cut sets were found to be in agreement.

Furthermore, the dominant NUREG/CR-4550 T1 accident sequence cut sets, requantified with the revised fault trees, were in agreement with the same accident sequences in the IPE; therefore, the IPE TAC2 accident sequences appear to have been properly incorporated into the NUREG/CR-4550 model.

\subsubsection{PRA Model for Low Power Operations (POS 1)}

In POS 1, the plant is in a low power condition (i.e., thermal power $\leq 15 \%$ at rated pressure $\approx 1000 \mathrm{psig}$ ) and, in this study, is assumed to be shutting down for refueling. Since the plant is still at power in POS 1 and not shutdown, it was assumed that the conditions in POS 1 could be modeled using the POS 0 full power model. The only difference between the POS 0 model and the POS 1 model is the initiating event frequency for T1 $(0.1 / \mathrm{yr}$ in POS 0 and 0.07 in POS 1). Thus, the POS 1 results are the POS 0 results with the initiating frequency for $\mathrm{T} 1$ changed from $0.1 / \mathrm{yr}$ to $0.07 / \mathrm{yr}$.

\subsubsection{PRA Model for POSs $2 \& 3$}

In POS 2 and POS 3 the plant is in hot shutdown with all rods inserted into the core. In this analysis, it is assumed that the plant is shutting down for refueling. The Reactor Core Isolation Cooling (RCIC) system is still available for injection in both POS 2 and POS 3.

It was assumed that both POS 2 and POS 3 could be modeled as one utilizing the POS 0 model with minor revisions. These revisions included:

1) eliminating the Anticipated Transient Without Scram (ATWS) accident sequences since the plant is shutdown,

2) eliminating $T 1$ sequences 17 and 21 because it was assumed that the SRVs would not open for Reactor Coolant System (RCS) over- pressurization protection in POS 2 or POS 3 making stuck open relief valve failures invalid (i.e, no pressure surge in the vessel due to reactor scram), and

3) revising the initiating event frequency for $\mathrm{T} 1$ from $0.1 / \mathrm{yr}$ to $0.13 / \mathrm{yr}$. 


\subsubsection{PRA Model for POS 4}

POS 4 is a transition phase between POS 3 and POS 5 where RCIC is no longer assumed to be available (RCIC is declared inoperable at $135 \mathrm{psig}$ ), and the operator is switching to SDC for reactor core cooling. Because core cooling is being achieved with SDC, POS 4 appears to be similar to POS 5; therefore, the PRA model for POS 4 is a revised model of the PRA model for POS 5, i.e., the LP\&SD (Phase 2) model. Two major revisions to the POS 5 model were made to develop a POS 4 model:

1) the elimination of all sequences where Alternate Decay Heat Removal system (ADHRS) is initially operating providing core cooling (ADHRS cannot successfully cool the core in POS 4)

2) the elimination of all sequences where the containment is initially open; it is assumed that the containment is not yet opened for maintenance in POS 4.

\subsubsection{PRA Model for POS 5}

The PRA model used for POS 5 was developed in Phase 2 of the LP\&SD study at Sandia National Laboratories (SNL). No revisions to the event tree models were made since these models included all applicable mitigating systems even though the study assumed all Division/Train A systems were out for scheduled maintenance. Because the study did not give credit for Division/Train A systems, some of the fault tree models for these systems had to be developed and/or existing fault trees from NUREG/CR-4550 had to be revised to capture shutdown configurations not found at full power. Because this study did give credit for Division/Train A systems, recovery actions used in the LP\&SD study were also revised to include credit for the recovery of Division/Train A systems.

In order to perform this analysis, certain general assumptions had to be made pertaining to the use of the LP\&SD study results. These assumptions were as follows:

Either SDC Train B or ADHRS is initially in operation prior to the initiating events considered. Because either SDC Train B or ADHRS was assumed to be initially operating prior to an initiating event, neither these systems nor their supports could be assumed to be in scheduled maintenance for this analysis.

- $\quad$ All operator actions as quantified for the LP\&SD study apply in this analysis, i.e., operator actions were not requantified for this analysis.
- $\quad$ RCIC is not a viable injection source because it is not required to be operational by the TSs with a vessel pressure below 135 psig.

All recovery actions (i.e., operator actions) as quantified in the LP\&SD study apply in this analysis. However, since more systems are available in this analysis, the recovery actions were updated to reflect the difference in the assumed plant configuration.

Control Rod Drive system (CRD) Train A is assumed to be initially operating in makeup mode with the Reactor Water Cleanup System (RWCU) operating in letdown mode.

\subsubsection{PRA Model for POS 6}

In POS 6 the methods utilized by the operator to cool the core given a loss of SDC, as directed by procedure, are different from those used by the operator in POS 5. Therefore, the POS 6 PRA model could not be extrapolated from the POS 5 PRA model.

The PRA models developed for the LP\&SD (Phase 1) were used for POS 6. This study categorized accident sequences as "high," "medium, and "low" contributors to core damage frequency. In this analysis, only those sequences categorized as potentially high core damage frequency accidents were considered.

It should be noted that all of the operator actions in the screening analysis were given a human error probability of 1.0; however, for this analysis, all of the applicable operator actions were given an appropriate human error probability based on similar operator actions quantified in the LP\&SD study for POS 5. Also note that the screening analysis did not perform a recovery analysis for the surviving accident sequences, while in this analysis, recovery actions were applied where appropriate based on similar actions applied in the LP\&SD study for POS 5.

In order to perform this analysis, certain general assumptions had to be made pertaining to the use of the LP\&SD study results. These assumptions were as follows:

Either SDC Train B or ADHRS is initially in operation prior to the initiating events considered. Because either SDC Train B or ADHRS was assumed to be initially operating prior to an initiating event, neither these systems nor their supports could be assumed to be in scheduled maintenance for this analysis. 
The Safety Relief Valves (SRVs) and RCIC are not available in POS 6 since the vessel head is off.

CRD Train A is assumed to be initially operating in makeup mode with RWCU operating in letdown mode.

\subsubsection{PRA Model for POS 7}

For POS 7, the PRA models developed for the LP\&SD (Phase 1) were used in this analysis. As in POS 6, only those sequences categorized as potentially high $\mathrm{CDF}$ accidents in the LP\&SD (Phase 1) study were considered.

It should be noted that all operator actions in the screening analysis were given a human error probability of 1.0 , while in this analysis, all applicable operator actions were given an appropriate human error probability based on similar operator actions quantified in the analysis for POS 5.

In order to perform this analysis, certain general assumptions had to be made pertaining to the use of the LP\&SD study results. These assumptions were as follows:

Either SDC Train B or ADHRS is initially in operation prior to the initiating events considered. Because either SDC Train B or ADHRS was assumed to be initially operating prior to an initiating event, neither these systems nor their supports could be assumed to be in scheduled maintenance for this analysis.

The SRVs and RCIC are not available in POS 7 since the vessel head is off.

CRD Train A is assumed to be initially operating in makeup mode with RWCU operating in letdown mode.

Even though separate models were used to represent the eight POSs, the models were done in sufficient detail to allow for comparisons among the POSs.

\subsection{Model Evaluations}

The risk measures defined in Section 2.1 along with the PRA models developed in Section 2.2 were used to evaluate the risk impact of test and maintenance activities at power and shutdown. The PRA models were quantified using the IRRAS code, Version 5.1 [Russell et al., 1991]. The results of the raw data obtained for the evaluation of maintenance activities are listed in Appendix A. The results of the raw data obtained for the evaluation of surveillance tests are listed in Appendix B. 


\subsection{Results of the Risk Impact of Maintenance Activities}

This section of the report presents the results of the risk impact of single and multiple train maintenance on various systems during power operation, shutdown, and refueling. The systems include the Emergency Diesel Generators (EDGs), the Standby Service Water (SSW) system, the Batteries (BATTs), the RCIC system, the Emergency Core Cooling System (ECCS), and the Residual Heat Removal (RHR) system and are described in detail in Appendix E.

For core damage probabilities, the results are categorized as:

$$
\begin{aligned}
& \text { large }>1 \mathrm{E}-4 \\
& \text { medium } 1 \mathrm{E}-6 \text { to } 1 \mathrm{E}-4 \\
& \text { small } 1 \mathrm{E}-8 \text { to } 1 \mathrm{E}-6 \\
& \text { negligible }<1 \mathrm{E}-8 .
\end{aligned}
$$

Similarly, core damage frequencies are categorized as:

$$
\begin{aligned}
& \text { high }>1 \mathrm{E}-4 / \mathrm{yr} \\
& \text { medium } 1 \mathrm{E}-6 / \mathrm{yr} \text { to } 1 \mathrm{E}-4 / \mathrm{yr} \\
& \text { low } 1 \mathrm{E}-8 / \mathrm{yr} \text { to } 1 \mathrm{E}-6 / \mathrm{yr} \\
& \text { negligible } \quad<1 \mathrm{E}-8 / \mathrm{yr} \text {. }
\end{aligned}
$$

\subsection{Risk Impact of Emergency Diesel Generator (EDG) Maintenance Outage}

GGNS utilizes three EDGs. Division 1 EDG (EDG I) and division 2 EDG (EDG II) provide emergency power to division 1 and 2 Engineered Safety Features (ESF)

following an LOSP. Division 3 EDG (EDG III) is dedicated to the High Pressure Core Spray System (HPCS). The division 3 EDG can also be cross-tied to division 1 or 2 to provide AC power when EDG I or EDG II fails [USNRC, 1990]. Sections 3.1.1 and 3.1.2 summarize the risk impact of single and multiple EDG maintenance, respectively, and Sections 3.1.1.1 and 3.1.2.1 provide insights on single and multiple EDG maintenance activities.

\subsubsection{Single Train Maintenance on the EDGs}

The results of the $I_{\mathrm{CDFs}}$ for the EDGs (EDG I, EDG II, and EDG III), in all seven POSs, are listed in Appendix C and are shown graphically in Figure 9. The results indicate that there is a medium to high $\mathrm{I}_{\mathrm{CDF}}$ during low power and the first few days of hot and cold shutdown (POS 2, 3, 4 and 5) which is comparable to that at power. This is due to the fact that during low power, hot shutdown and the early stages of cold shutdown, the decay heat is still significant, the coolant inventory is limited, and except for RCIC in POS 1 through 3, only electric pumps are available for cooling. In addition, at shutdown, the frequency of one of the most important accidents, LOSP (T1), increases from $0.11 / \mathrm{yr}$ to $0.13 / \mathrm{yr}$. Therefore, in case of an accident involving an LOSP, the EDGs are essential for power provision. The $\mathrm{I}_{\mathrm{CDF}}$ with the plant operating in refueling, however, is substantially reduced especially in POS 7 where the decay heat is low and water level is high.

The results of the $I_{C D P s}$ and the $A_{1 C D F s}$ associated with all three EDGs are listed in Appendix $C$. The $I_{C D P s}$ are based on AOTs of 72 hours for EDG I and II and 408 hours for EDG III with the plant operating at power and low power. An AOT of 24 hours for all three EDGs is used when the plant is operating in hot shutdown, and an AOT equivalent to the time spent in the POS is used for cases where an AOT is not specified at shutdown. The results indicate that $I_{C D P s}$ for the EDGs in POSs 1, 2, 3, 4, and 5 are comparable to that at power and are in the small to medium range. However, they are substantially reduced (small to negligible) with the plant operating in refueling (POS 6 and POS 7). The results of the $A_{\text {ICDFs }}$ are based on a maintenance frequency of $2.6 / \mathrm{yr}$. These results indicate that the $\mathrm{A}_{\mathrm{ICDFs}}$ are low for power operations. A time profile of the change in core damage frequency for taking an EDG out for maintenance is shown in Figure 10. (Note that the time axis is an estimate of the number of days in an operating cycle). 
Risk Impact of Maintenance

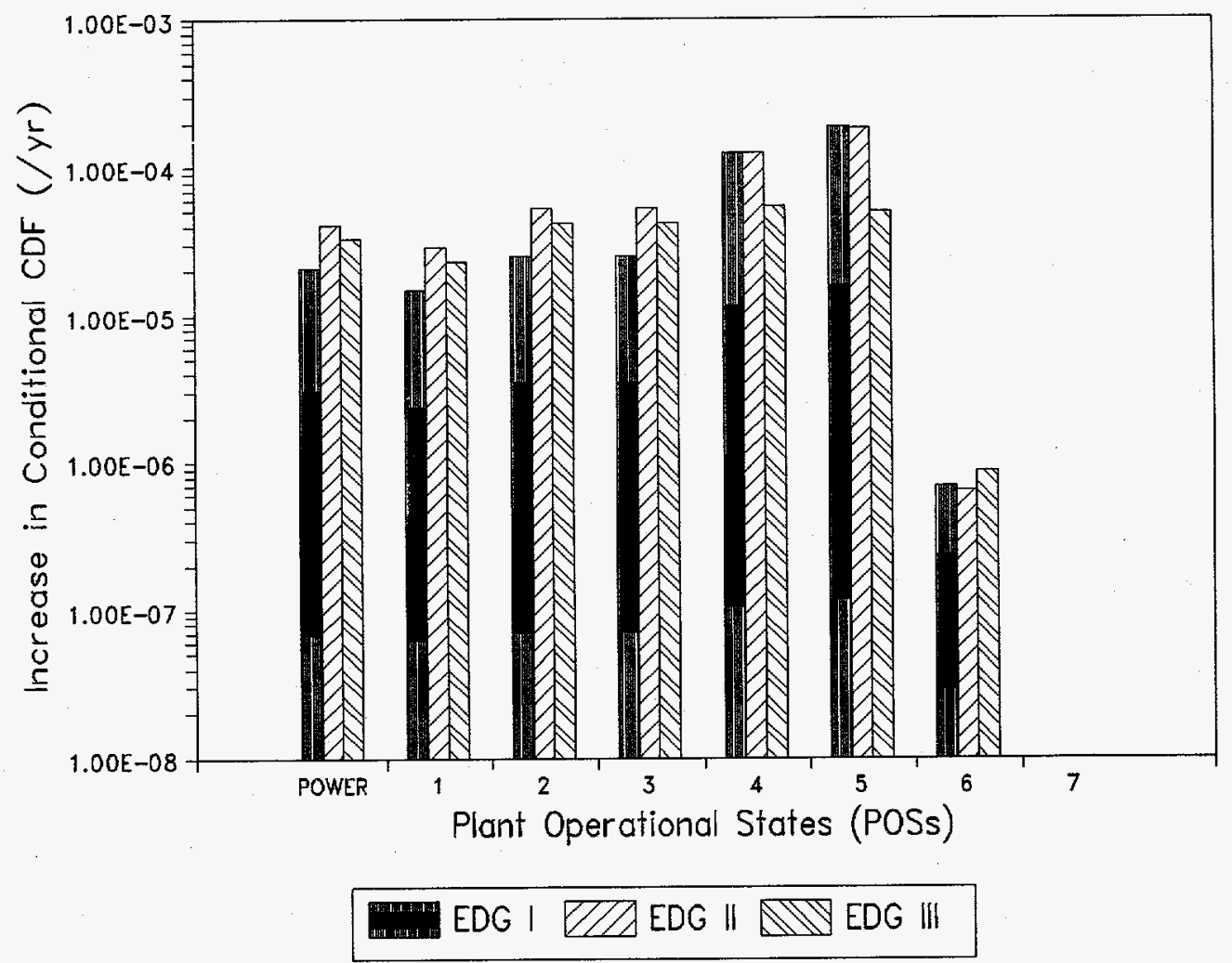

Figure 9. Increase in Conditional Core Damage Frequency for Single EDG Maintenance 


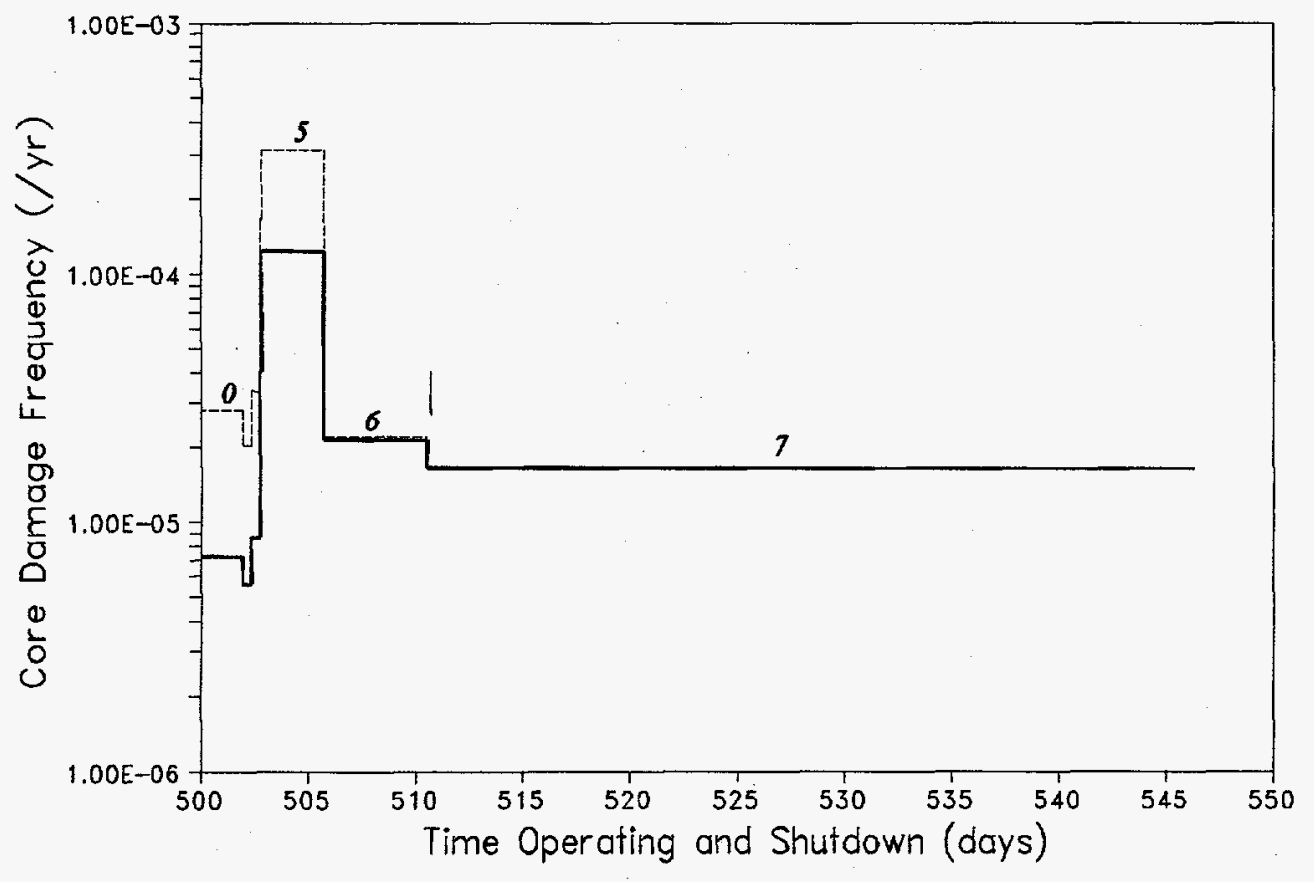

- No Mainfenance ----- EDG in Maintenance

Figure 10. Change in Core Damage Frequency for Taking a Single EDG Out for Maintenance During the Different POSs in an Operating Cycle 


\subsubsection{Insights on Single EDG Maintenance}

The results discussed above provide the following insights regarding the scheduling of EDG maintenance outages:

Even though the $I_{C D F}$ is medium for taking a single EDG out of service at power, preventative maintenance of a few days (e.g., 3 days for EDG II) can be scheduled with only a small increase in the probability of core damage and $A_{\mathrm{ICDF}}$. However, repeated use of the LCOs to perform preventative maintenance on the EDGs at power could significantly increase the $A_{\mathrm{ICDF}}$. For example, increasing the maintenance frequency, for EDG I, from 2.6/yr to $6 / \mathrm{yr}$ changes the $A_{\text {ICDF }}$ to $1.03 \mathrm{E}-6 / \mathrm{yr}$ ( $\approx 14 \%$ of the baseline core damage frequency).

Based on the $\mathrm{I}_{\mathrm{CDF}}$, there appears to be no risk advantage in performing maintenance on a single EDG during low power and early stages of shutdown.

Based on the $\mathrm{I}_{\mathrm{CDF}}$, preventative maintenance of long or uncertain duration (e.g., EDG overhauls) should preferably be scheduled for refueling (POSs 6 or 7). Furthermore, for EDG I, the Outage Time at Refueling (OTR) in POS 6 that gives a core damage probability comparable to that at power (1.73E-7) is approximately 93 days. This indicates that EDG I could possibly be out for a much longer duration if maintained at refueling as opposed to power.

\subsubsection{Multiple Train Maintenance on the EDGs}

The $I_{\mathrm{CDFs}}$ for multiple division maintenance on the EDGs ate listed in Appendix $\mathrm{C}$ and are shown graphically in Figure 11. The results indicate that there is a high $\mathrm{I}_{\mathrm{CDF}}$ during low power and the first few days of hot and cold shutdown (POS $2,3,4$ and 5) which is comparable to that at power. However, the $I_{\mathrm{CDF}}$ with the plant operating in refueling is substantially lower.

The results of the $I_{\text {CDPs }}$ and the $A_{I C D F}$ for power operations are listed in Appendix $C$. The $I_{C D P s}$ are based on AOTs of 2 hours with the plant operating at power and low power. An AOT of 24 hours is used for the multiple EDG outage configuration when the plant is operating in hot shutdown, and an AOT equivalent to the time spent in the POS is used for cases where an AOT is not specified at shutdown. The results indicate that $I_{\mathrm{CDPs}}$ for the multiple
EDG outage configurations in POSs 1, 2, 3, 4, and 5 are comparable to that at power and are in the small to medium range. However, the $I_{\mathrm{CDPs}}$ with the plant operating in refueling (POS 6 and POS 7) are substantially lower (especially in POS 7). The $A_{I C D F}$ at power is based on a maintenance frequency of $0.03 / y$ r. The results show that the $A_{\mathrm{ICDF}}$ is low to negligible.

\subsubsection{Insights on Multiple EDG Maintenance}

The results discussed above provide the following insights regarding the scheduling of multiple EDG maintenance outages:

The Increase in Conditional CDF for multiple EDG maintenance activities appear to be more significant than that of a single EDG.

- Based on the $I_{C D F}$, there appears to be no risk advantage in performing maintenance on multiple EDGs during the early stages of shutdown.

Based on the $\mathrm{I}_{\mathrm{CDF}}$, multiple preventative maintenance of long or uncertain duration should preferably be scheduled for refueling (POSs 6 or 7). Furthermore, for simultaneous maintenance on EDG I and II, the OTR in POS 6 that gives a core damage probability comparable to that at power $(7.05 \mathrm{E}-8)$ is approximately 2 days. This indicates that simultaneous maintenance on EDGs I and II could possibly be performed for a longer duration if the maintenance is done at refueling as opposed to power.

\subsection{Risk Impact of Stendby Service Water (SSW) Maintenance Outage}

At GGNS there are three independent trains of SSW: Trains A, B, and C. Train A provides room cooling for Low Pressure Core Spray (LPCS), RCIC, and RHR train A (RHR A), EDG I jacket cooling and RHR A pump and heat exchanger cooling. Train B provides room cooling for RHR trains B and C, EDG II jacket cooling, and RHR B pump and heat exchanger cooling. Train $\mathrm{C}$ is dedicated to HPCS and provides EDG III jacket cooling. The risk impact of single and multiple train maintenance on SSW are discussed in sections 3.2.1 through 3.2.3, and Sections 3.2.1.1, 3.2.2.1 and 3.2.3.1 provide insights on single and multiple train SSW maintenance activities. 


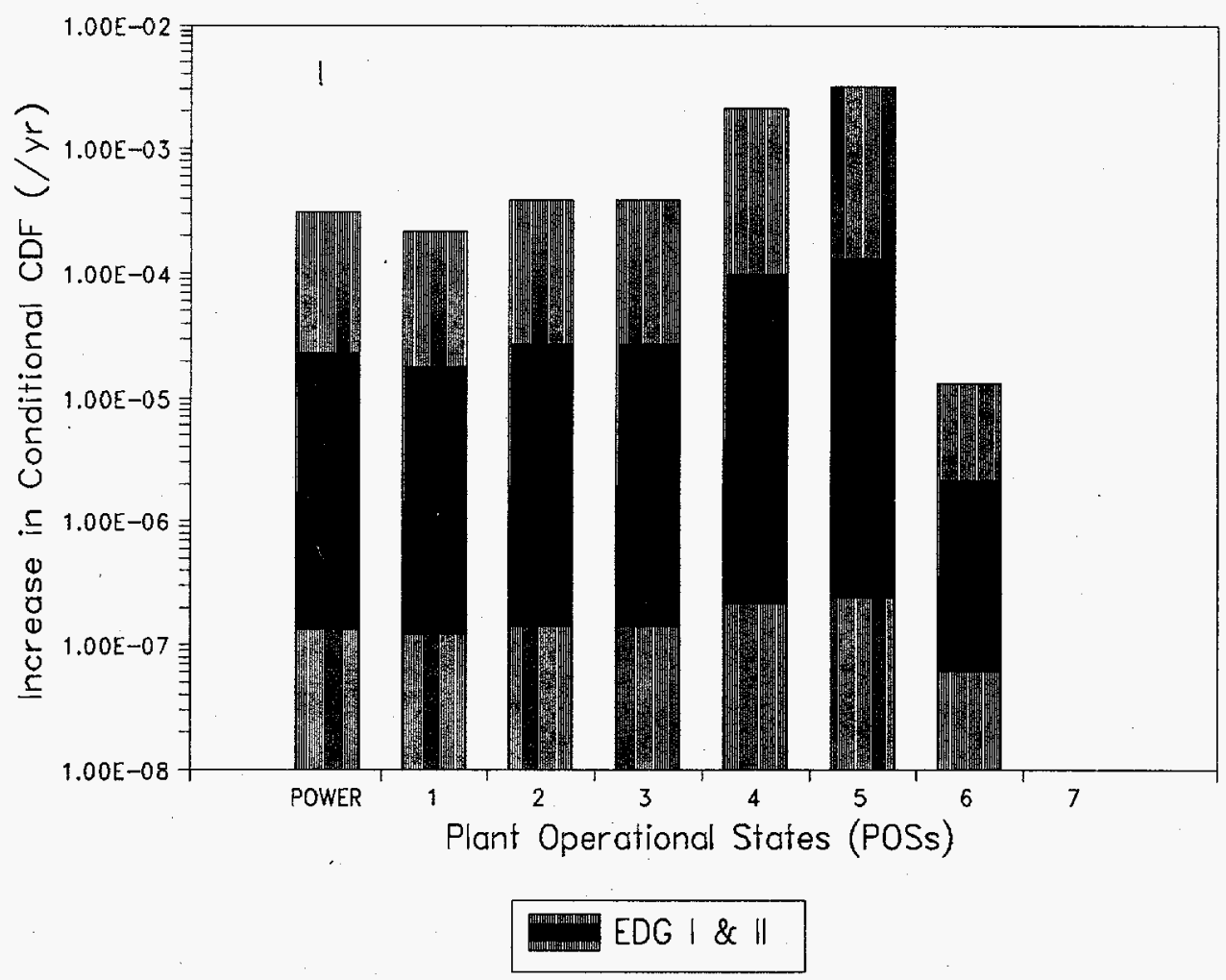

Figure 11. Increase in Conditional Core Damage Frequency for Multiple EDG Maintenance

\subsubsection{Single Train Maintenance on the SSW}

The $I_{\mathrm{CDFs}}$ for a single train of SSW (SSW A) in all seven POSs, are listed in Appendix $\mathrm{C}$ and are shown graphically in Figure 12. The results indicate that there is a medium $I_{\mathrm{CDF}}$ during low power and the first few days of hot shutdown (POS 2 and 3) which is comparable to that at power. For POSs 4 and 5, the $\mathrm{I}_{\mathrm{CDF}}$ increases to the high range. This is due to the fact that, at power, the dominant accident sequences are LOSP sequences (T1) which require the EDGs to perform with SSW cooling. 


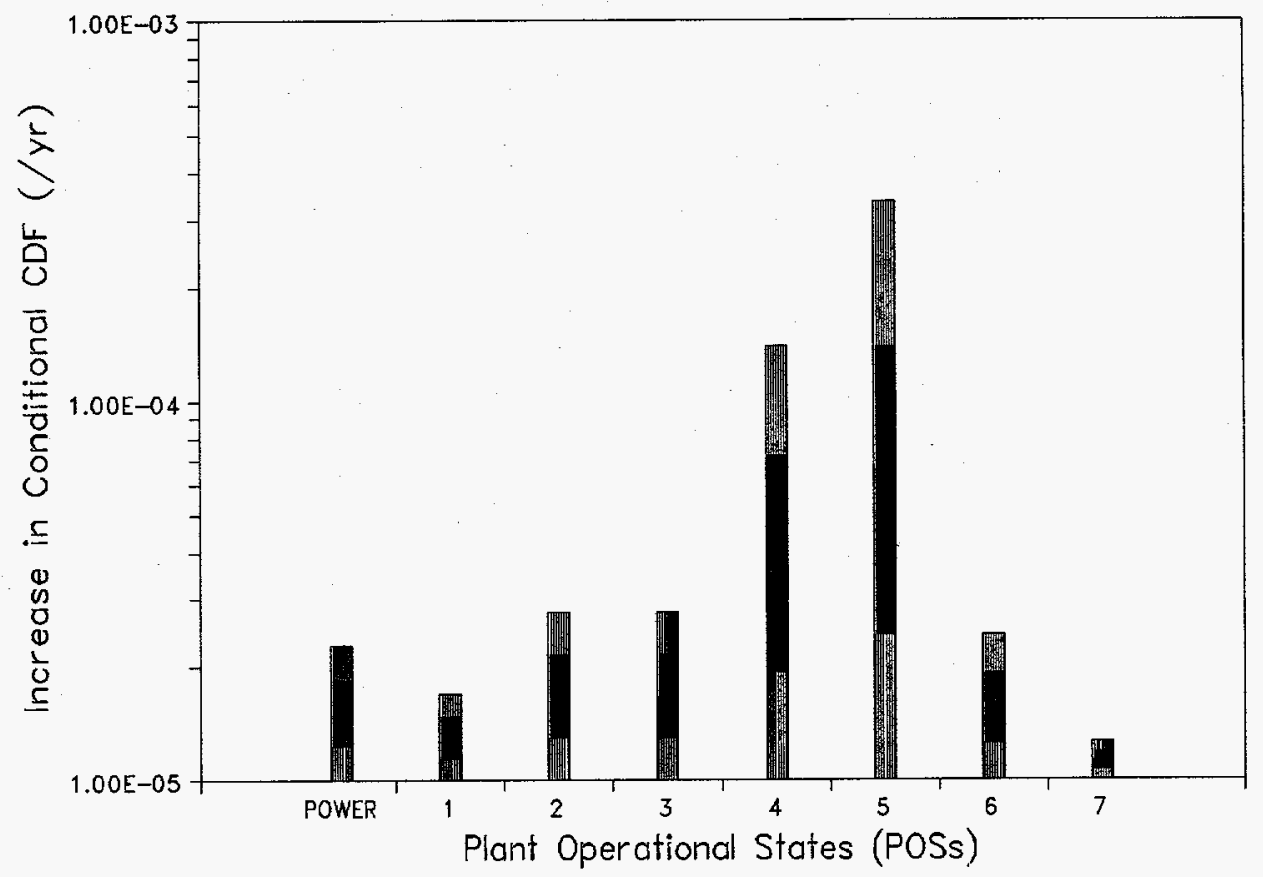

Figure 12. Increase in Conditional Core Damage Frequency for Single Train SSW Maintenance

At shutdown, POS 4 and 5 , the decay heat is still relatively high, but the condenser vacuum is not sufficient to remove heat as in POSs 2 and 3 and so the plant normally shifts to Decay Heat Removal (DHR). In addition, the dominant accidentsequences are associated with a loss of DHR and an LOSP. Since SSW is needed to provide cooling support for
DHR, and in cases of a LOSP, SSW is needed for proper operation of the EDGs, its importance increases at shutdown. The $\mathrm{I}_{\mathrm{CDF}}$ is reduced, compared to power, with the plant operating in refueling (POS 7) where the decay heat is low and the dependence on SSW is reduced. 
Risk Impact of Maintenance

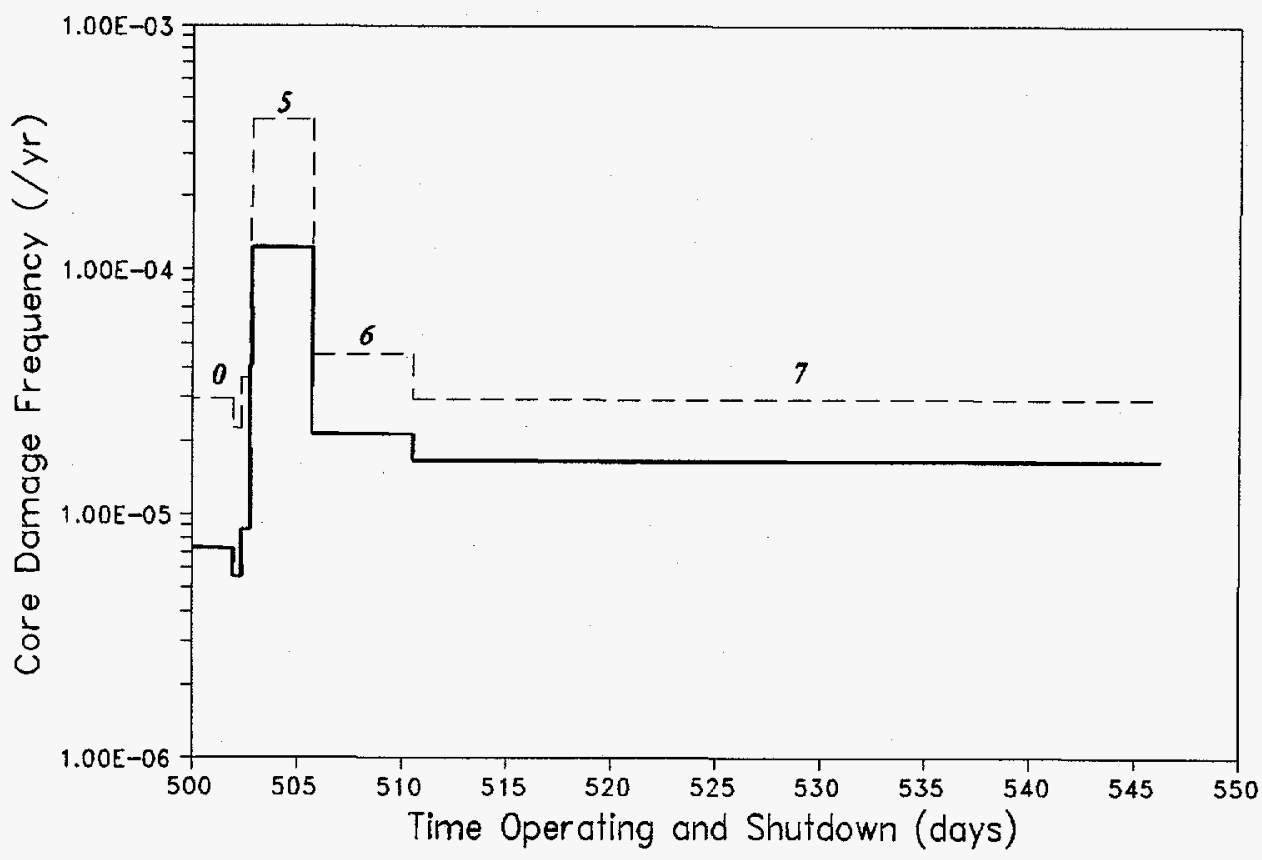

No Maintenance - - SSW in Maintenance

Figure 13. Change in Core Damage Frequency for Taking a Train of SSW Out for Maintenance During the Different POSs in an Operating Cycle

The $\mathrm{I}_{\mathrm{CDPs}}$ and the $\mathrm{A}_{\mathrm{ICDFs}}$ are listed in Appendix $\mathrm{C}$. The $\mathrm{I}_{\mathrm{CDPs}}$ are based on AOTs of 72 hours for SSW A with the plant operating at power and low power. An AOT of 24 hours is used when the plant is operating in hot shutdown, and an AOT equivalent to the time spent in the POS is used for cases where an AOT is not specified at shutdown. The results indicate that $I_{C D P s}$ for SSW A in POSs $1,2,3,4$, and 5 are comparable to that at power and are in the small to medium range. The $I_{\mathrm{CDPs}}$ with the plant operating in refueling (POS 6 and POS 7) are small. The results of the $A_{I C D F s}$ are based on a maintenance frequency of $0.88 / y r$. These results indicate that the $A_{\mathrm{ICDFs}}$ are low for power operations. A time profile of the change in core damage frequency for taking a single train of SSW out for maintenance is shown in Figure 13.

\subsubsection{Insights on Single SSW Train Maintenance}

The results discussed above provide the following insights regarding the scheduling of single SSW train maintenance outages:

Even though the $\mathrm{I}_{\mathrm{CDF}}$ for single train maintenance on SSW is medium at power, preventative maintenance of a few days (e.g., 72 hours for SSW A) can be scheduled with only a small increase in the 
probability of core damage. However, repeated use of the LCOs to perform preventative maintenance on SSW at power could significantly increase the $A_{\mathrm{ICDF}}$. For example, increasing the maintenance frequency, for SSW A, from $0.88 / \mathrm{yr}$ to $6 / \mathrm{yr}$ changes the $\mathrm{A}_{\mathrm{ICDF}}$ to $1.11 \mathrm{E}-6 / \mathrm{yr}(\approx 15 \%$ of the baseline core damage frequency).

ADHRS, which is cooled by Plant Service Water (PSW) at GGNS, can only be used 24 hours after shutdown, thus, planned maintenance should preferably not be scheduled for the late phases of hot shutdown and the early phases of cold shutdown, when ADHRS is not an alternative for decay heat removal, unless the operability of an alternate train of DHR is confirmed. For example, if SSW A is out for maintenance, SSW B would be needed to cool RHR train B at shutdown, especially in POS 4 where RHR train B is the only available non-emergency means of decay heat removal.

The $\mathrm{I}_{\mathrm{CDF}}$ at refueling is only slightly less than that at power. Therefore, there seems to be only a small advantage to performing single train SSW maintenance of longer than 3 days at refueling as compared to power. Furthermore, for SSW A, the OTR, in POS 7, that gives a core damage probability comparable to that at power (1.85E-7) is approximately 6 days.

\subsubsection{Multiple Train Maintenance on SSW A \& SSW C}

The $I_{\text {CDFs }}$ for multiple maintenance on SSW trains A and C are listed in Appendix $\mathbf{C}$ and are shown graphically in Figure 14. The results indicate that there is a high $\mathrm{I}_{\mathrm{CDF}}$ during low power and the first few days of hot and cold shutdown (POSs 1, 2, 3, 4 and 5) which is comparable to that at power. However, unlike the EDGs, the $I_{\mathrm{CDF}}$ with the plant operating in refueling (POS 6 and 7) remains in the high range.

The $\mathrm{I}_{\mathrm{CDP} \mathrm{s}_{\mathrm{s}}}$ and the $\mathrm{A}_{\mathrm{ICDF}}$ are listed in Appendix $\mathrm{C}$. The $\mathrm{I}_{\mathrm{CDPs}}$ are based on AOTs of 72 hours with the plant operating at power and low power. The 72 hour AOT is not listed as a TS requirement; however, 72 hours is an upper bounding time limit associated with the SSW A outage. An AOT of 24 hours is used for the multiple SSW train outage configurations when the plant is operating in hot shutdown, and an AOT equivalent to the time spent in the POS is used for cases where an AOT is not specified at shutdown. The results indicate that $\mathrm{I}_{\mathrm{CDPs}}$ for the SSW trains $\mathrm{A}$ and $\mathrm{C}$ outage configurations in POSs $1,2,3,4$, and 5 are comparable to that at power and are in the medium range. The $\mathrm{I}_{\mathrm{CDPs}}$ with the plant operating in refueling (POS 6 and POS 7) are in the small to medium range. The results also indicate that the $A_{I C D F}$ is low for power operations.

\subsubsection{Insights on SSW Trains A \& C Maintenance}

The results discussed above provide the following insights regarding the scheduling of multiple SSW train maintenance outages:

- The Increase in Conditional CDF for SSW trains A and $C$ maintenance activities appears more significant than that of a single SSW train.

Maintenance should preferably not be scheduled for the late phases of hot shutdown and the early phases of cold shutdown, when ADHRS is not an alternative for decay heat removal, unless the operability of SSW B is confirmed. With SSW A out for maintenance, SSW B would be required to cool RHR train B at shutdown, especially in POS 4 where RHR train $B$ is the only available nonemergency means of decay heat removal.

The $\mathrm{I}_{\mathrm{CDF}}$ at refueling is significantly smaller than that at power. Thus, performing SSW trains $A$ and $C$ maintenance in refueling seems to be advantageous. Furthermore, for multiple maintenance on SSW A and C, the OTR in POS 6 that gives a core damage probability comparable to that at power (2.81E-5) is approximately 25 days. This indicates that SSW A an C could possibly be out for a longer duration if maintained in refueling as opposed to power.

\subsubsection{Multiple Train Maintenance on SSW A \& SSW B}

The $I_{\text {CDFs }}$ for multiple maintenance on SSW trains A and B are listed in Appendix C. Since one train of RHR, which is supported by SSW train A or B, is normally operating for decay heat removal during shutdown, simultaneous maintenance on SSW trains A and B was not modeled in POSs $4,5,6$, and 7 . Thus, the quantitative analysis was limited to POSs 0 (power operations), 1, 2, and 3. Qualitative arguments are presented for POSs 4 through 7. The quantitative results indicate that there is a high $\mathrm{I}_{\mathrm{CDF}}$ during POSs 1,2 , and 3 which is comparable to that at power. For POS 4 , the decay heat is still relatively high, but the condenser vacuum is not sufficient to 


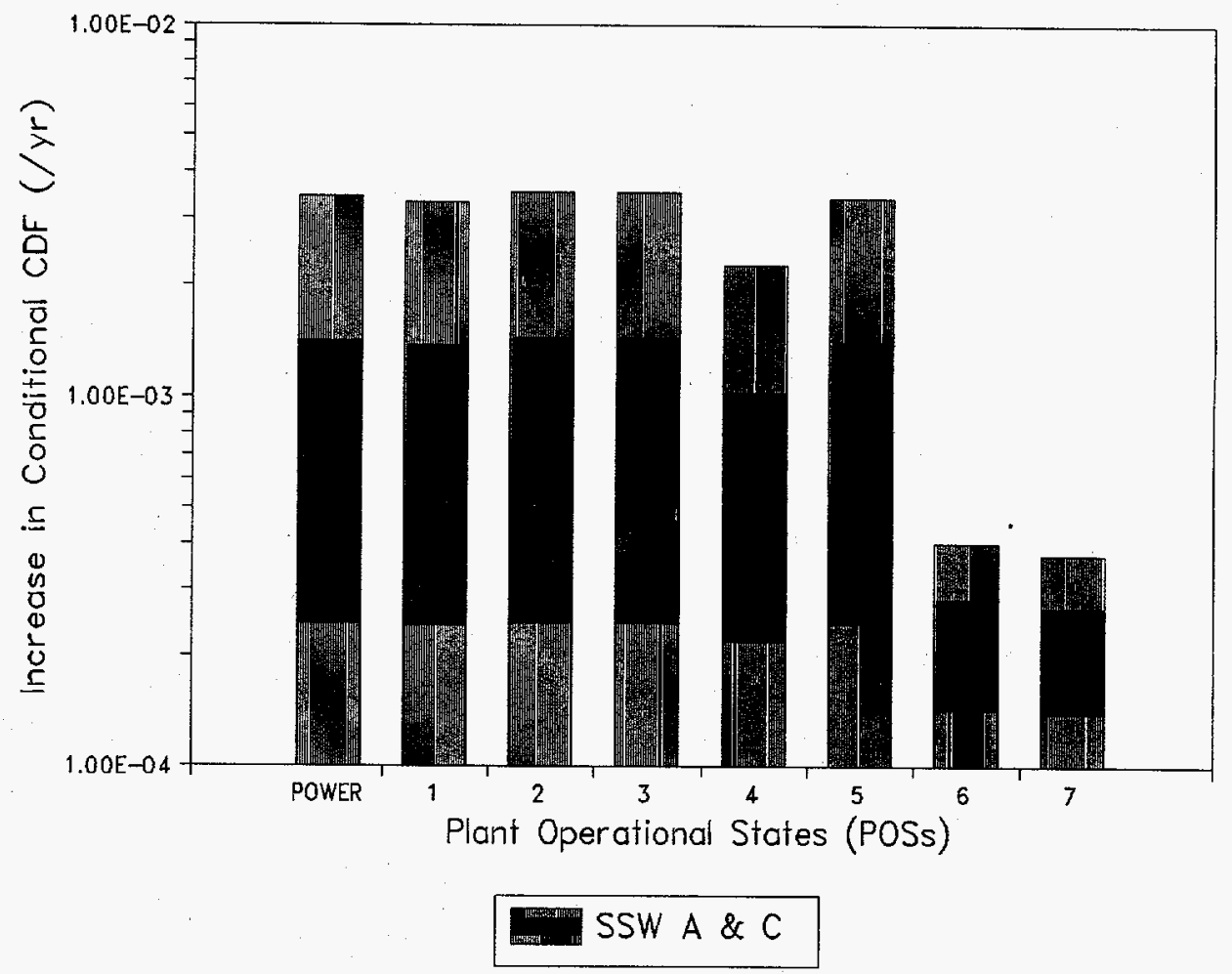

Figure 14. Increase in Conditional Core Damage Frequency for Multiple Train SSW Maintenance 
remove heat as in POS 2 and 3. Therefore, the plant normally shifts to RHR. However, if both trains A and B of SSW are unavailable, RHR is not an option for core cooling in the POS. In this case, the plant emergency procedures direct operators to flood the core with HPCS, open the SRVs, and dump steam to the suppression pool. The heat in the containment could then be controlled by venting. In POSs 5, 6 and 7, if SSW trains A and B are unavailable, core cooling can be accomplished using ADHRS.

The $\mathrm{I}_{\mathrm{CDPs}}$ for POS 0, 1, 2, and 3 are listed in Appendix $\mathrm{C}$. Since the TSs require an immediate shutdown following a discovery of concurrent SSW trains A and B failures, the AOT with the plant operating at power and low power is zero. An AOT of 24 hours is used for the SSW trains A and $B$ outage configurations when the plant is operating in POSs 2 and 3. The results indicate that $I_{\mathrm{CDPs}}$ for the SSW trains A and B outage configurations in POSs 2 and 3 are in the medium range $(5.5 \mathrm{E}-6 / \mathrm{yr})$. Defining an AOT of approximately 8 hours at power operations results in only a small increase in the probability of core damage (5.42E-7).

\subsubsection{Insights on SSW Trains A \& B Maintenance}

The results discussed above provide the following insights regarding the scheduling of SSW trains A and B maintenance outages:

The Increase in Conditional CDF for simultaneous maintenance on SSW trains A and B appear more risk significant than that of single SSW train maintenance activities.

Planned maintenance should preferably not be scheduled for the late phases of hot shutdown and the early phases of cold shutdown, when ADHRS is not an alternative for decay heat removal. Any such activity would require the use of emergency procedures.

Long term multiple maintenance on SSW trains A. and $B$, should preferably be done at refueling where the decay heat is low and ADHRS is available for DHR.

\subsection{Risk Impact of DC Power Batteries (BATTs) Maintenance Outage}

GGNS utilizes three Division Batteries (BATTs). Division 1 BATT (BATT I) and division 2 BATT (BATT II) provide emergency power to division 1 and 2 ESF loads, respectively. Division 3 BATT (BATT III) is dedicated to HPCS. Sections 3.3.1 and 3.3.2 summarize the risk impact of single and multiple BATT maintenance, and Sections 3.3.1.1 and 3.3.2.1 provide insights on single and multiple BATT maintenance activities.

\subsubsection{Single Train Maintenance on the BATTs}

The $\mathrm{I}_{\mathrm{CDFs}}$ for division 1, 2, and 3 BATTs (BATT I, BATT II, and BATT III) are listed in Appendix C and are shown graphically in Figure 15. The results indicate that there is a medium to high $\mathrm{I}_{\mathrm{CDF}}$ during low power and the first few days of hot and cold shutdown (POS 1, 2, 3, 4 and 5) which is comparable to that at power. However, the $\mathrm{I}_{\mathrm{CDF}}$ with the plant operating in refueling (POS 7) is substantially reduced as the decay heat is low and water level is high.

The $\mathrm{I}_{\mathrm{CDPs}}$ and the $\mathrm{A}_{\mathrm{ICDFs}}$ are listed in Appendix $\mathrm{C}$. The $\mathrm{I}_{\mathrm{CDPs}}$ are based on AOTs of 2 hours for BATTs I and II and 408 hours for BATT III with the plant operating at power and low power. An AOT of 24 hours for all three BATTs is used when the plant is operating in hot shutdown, and an AOT equivalent to the time spent in the POS is used for cases where an AOT is not specified at shutdown. The results indicate that $I_{\mathrm{CDPs}}$ for the BATTs in POSs $1,2,3,4$, and 5 are comparable to that at power and are in the small to medium range. The $I_{\text {CDPs }}$ with the plant operating in refueling (POS 6 and POS 7) are substantially reduced and are in the small to negligible range. The results of the $\mathrm{A}_{\mathrm{ICDFs}}$ are based on a maintenance frequency of $0.88 / \mathrm{yr}$. The results indicate that the $A_{\mathrm{ICDFs}}$ are low for power operations. A time profile of the change in core damage frequency for taking a single BATT out for maintenance is shown in Figure 16. 
Risk Impact of Maintenance

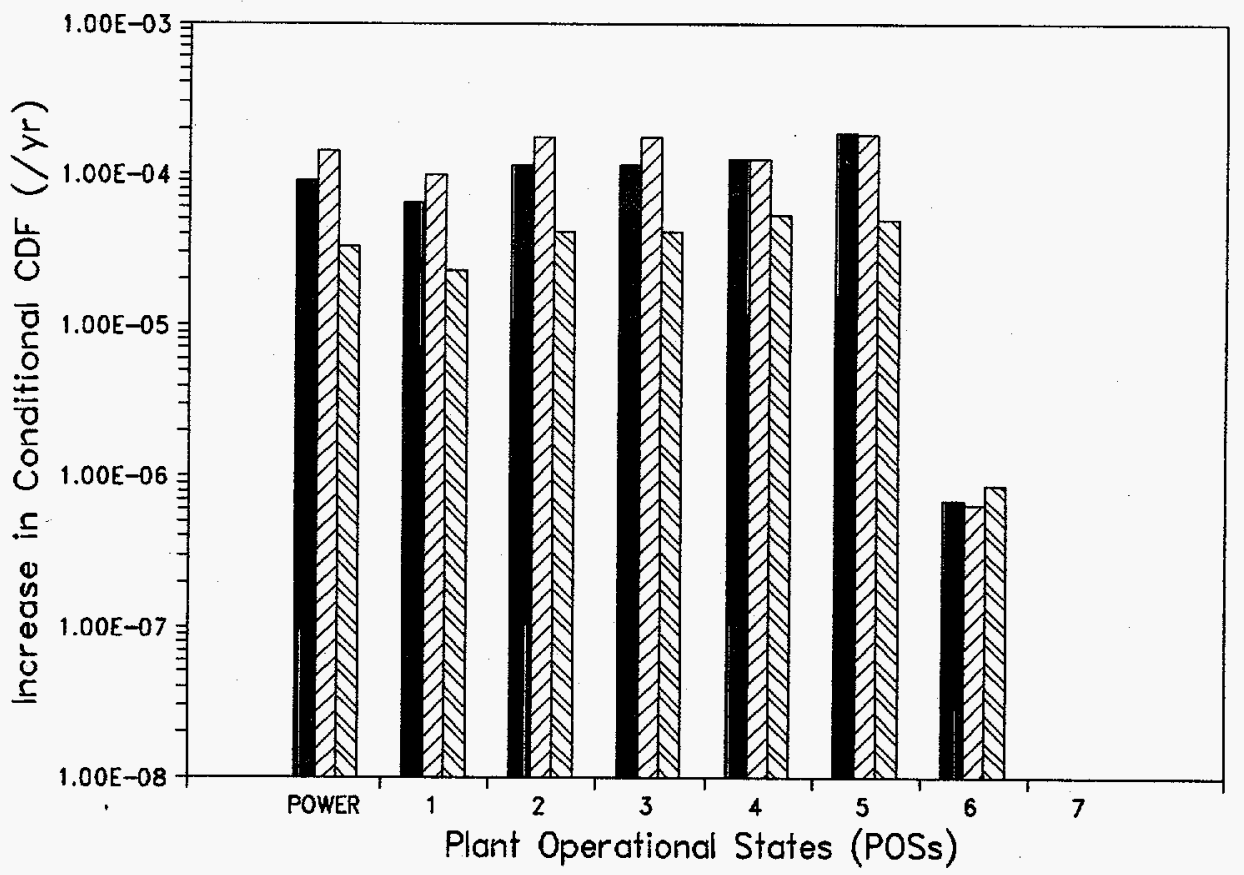

E. BATT I QZZ BATT ॥ M BATT II

Figure 15. Increase in Conditional Core Damage Frequency for Single Battery Maintenance 


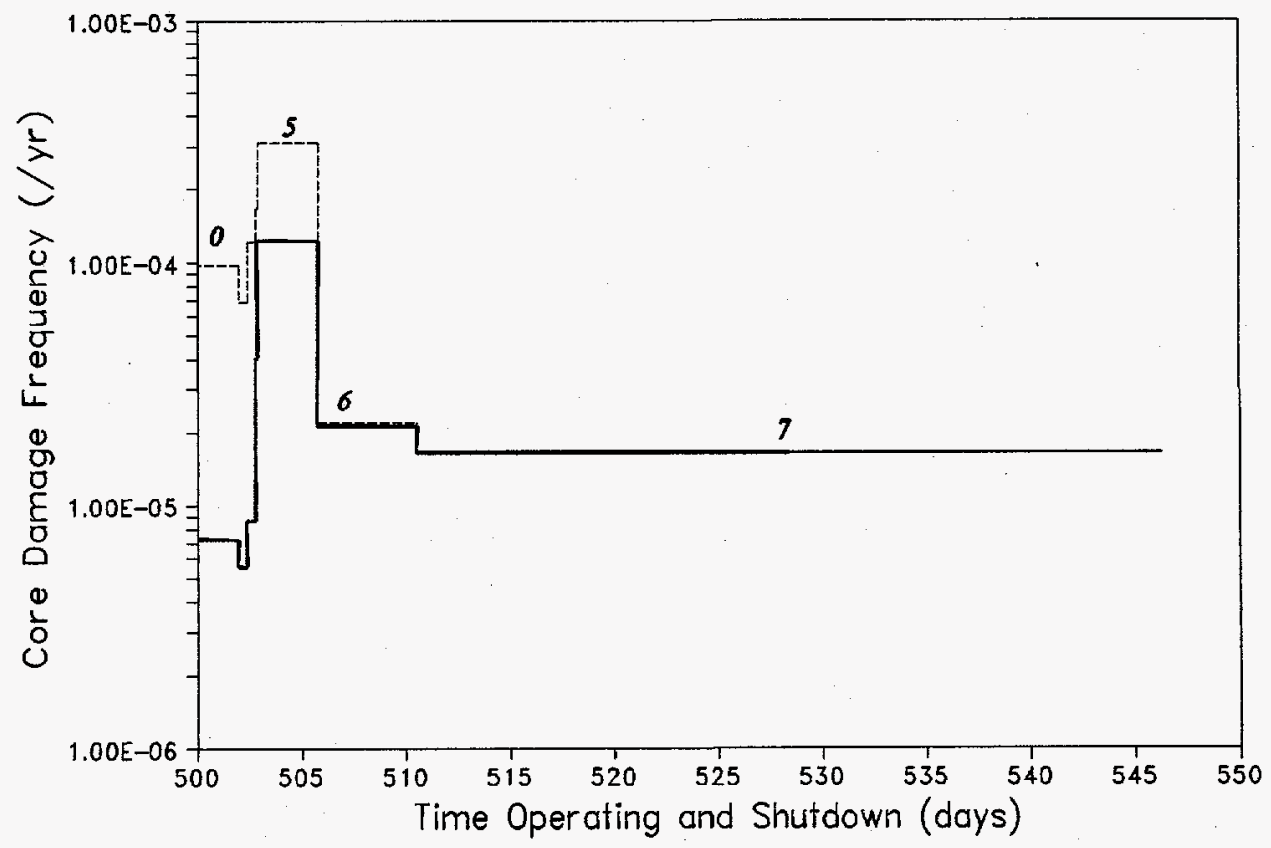

No Maintenance BATT in Maintenance

Figure 16. Change in Core Damage Frequency for Taking a Battery Out for Maintenance During the Different POSs in an Operating Cycle 


\subsubsection{Insights on Single Battery Maintenance}

The results discussed above provide the following insights regarding the scheduling of single BATT maintenance outages:

- Even though the $I_{C D F}$ for a single battery out of service at power is in the medium range, preventative maintenance of a few hours (less than 2 hours) can be scheduled at power operations with only a small increase in the probability of core damage (e.g., 3.2E-8 for BATT II). This small AOT of 2 hours appears to be reasonable as it moderates the high $\mathrm{I}_{\mathrm{CDF}}$ associated with a single BATT outage. However, since the increase in the probability of core damage is small, the possibility of extending the AOT might be considered without creating a substantial increase in the core damage probability. For example, if the AOT is extended from 2 to 8 hours, the core damage probability increases to 1.28E-7 (for BATT II) which is still low. This extension could allow more time for diagnosis and proper repair.

- $\quad$ By limiting the AOT to 2 hours, preventative maintenance on the batteries could probably be scheduled regularly at power without significantly increasing the $\mathrm{A}_{\mathrm{ICDF}}$. For example, by scheduling BATT II for maintenance 6 times per year, the $\mathrm{A}_{\mathrm{ICDF}}$ increases to $1.92 \mathrm{E}-7 / \mathrm{yr}(\approx 3 \%$ of the baseline core damage frequency).

Based on the $\mathrm{I}_{\mathrm{CDF}}$, there seems to be no risk advantage in performing maintenance on a single battery during the early stages of shutdown.

Based on the $I_{\mathrm{CDF}}$, preventative maintenance of long or uncertain duration (e.g., major BATT repair) should preferably be scheduled for refueling. Furthermore, for BATT II, the OTR in POS 6 that gives a core damage probability comparable to that at power (3.2E-8) is approximately 18 days. This indicates that BATT II could possibly be out for a much longer duration if maintained at refueling as opposed to power.

\subsubsection{Multiple Train Maintenance on the BATTs}

The $\mathbf{I}_{\mathrm{CDFs}}$ for multiple division maintenance on the BATTs are listed in Appendix $C$ and are shown graphically in
Figure 17. The results indicate that there is a high $\mathrm{I}_{\mathrm{CDF}}$ during low power and the first few days of hot and cold shutdown (POSs 1, 2, 3, 4 and 5) which is comparable to that at power. However, the $I_{C D F}$ with the plant operating in refueling is substantially reduced.

The $\mathrm{I}_{\mathrm{CDPs}}$ and the $\mathrm{A}_{\mathrm{ICDFs}}$ are listed in Appendix $\mathrm{C}$. The $\mathrm{I}_{\mathrm{CDPs}}$ are based on AOTs of 2 hours with the plant operating at power and low power. The 2 hour AOT is not listed as a TS requirement; however, 2 hours is an upper bounding time limit associated with a single BATT outage. An AOT of 24 hours is used for the multiple BATT outage configurations when the plant is operating in hot shutdown, and an AOT equivalent to the time spent in the POS is used for cases where an AOT is not specified at shutdown. The results indicate that $I_{\text {CDPs }}$ for the multiple BATT outage configuration in POSs $1,2,3,4$, and 5 are comparable to that at power and are in the small to medium range. The $I_{\text {CDPs }}$ with the plant operating in refueling (POS 6 and POS 7) are in the small to negligible range. The $A_{I C D F s}$ at power are based on a maintenance frequency of $1.75 \mathrm{E}-3 / \mathrm{yr}$. The results indicate that the $A_{\mathrm{ICDF}}$ are negligible for power operations.

\subsubsection{Insights on Multiple Battery Maintenance}

The results discussed above provide the following insights regarding the scheduling of multiple BATT maintenance outages:

The Increase in Conditional CDF for multiple BATT maintenance activities appear much more risk significant than that of single BATT maintenance activities.

Based on the $\mathrm{I}_{\mathrm{CDF}}$, there seems to be no risk advantage in performing maintenance on multiple batteries during the early stages of shutdown.

Based on the $I_{\mathrm{CDF}}$, multiple maintenance of long or uncertain duration should preferably be scheduled for refueling. Furthermore, for simultaneous maintenance on BATTs I and II, the OTR in POS 6 that gives a core damage probability comparable to that at power (2.6E-7) is approximately 7 days. This indicates that simultaneous maintenance on BATTs I and II could possibly be performed for a longer duration if the maintenance is done at refueling as opposed to at power. 
Risk Impact of Maintenance

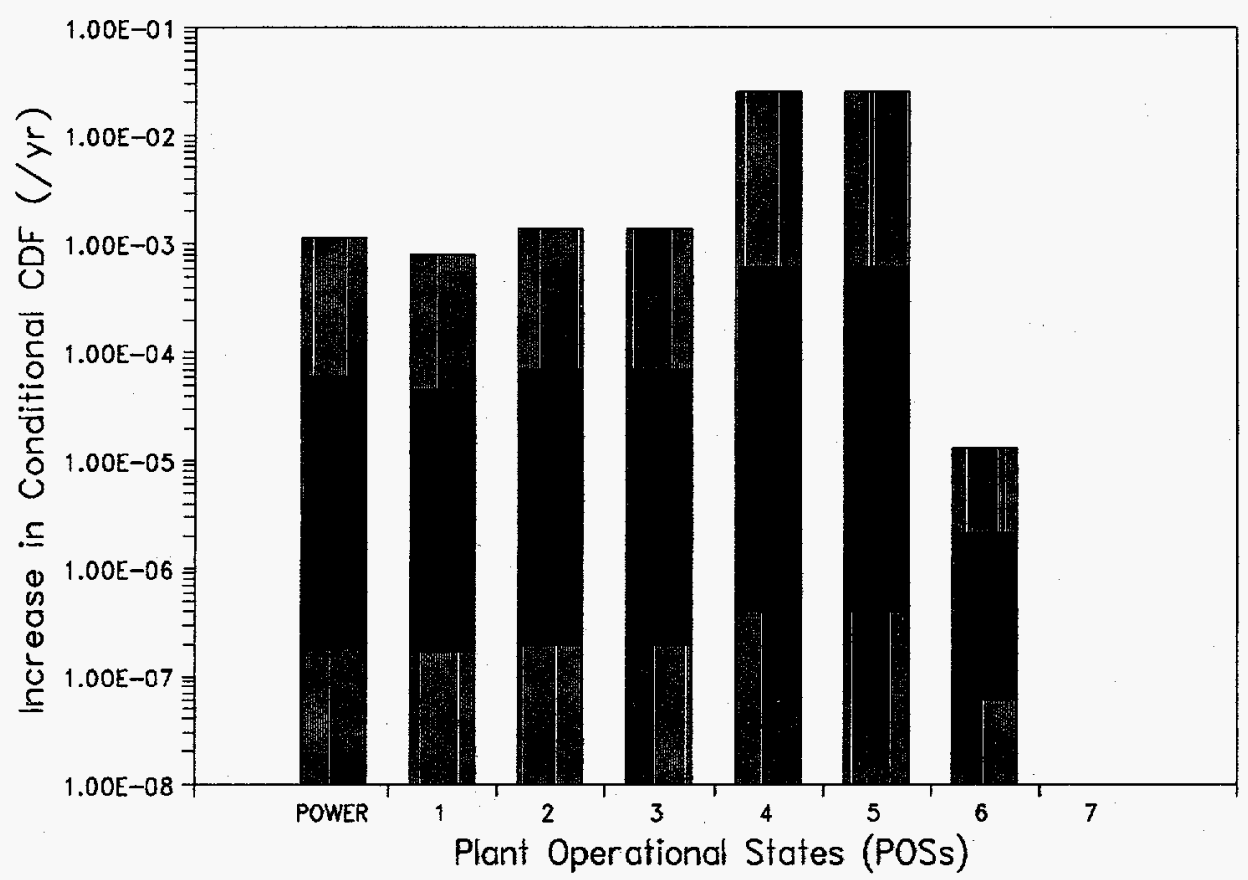

CBATT | \& II

Figure 17. Increase in Conditional Core Damage Frequency for Multiple Battery Maintenance 


\subsection{Risk Impact of Emergency Core Cooling System (ECCS) Maintenance Outage}

ECCS consists of HPCS, LPCS, and the Low Pressure Coolant Injection (LPCI) system. The HPCS system is a single train system which provides high pressure coolant injection to the reactor during accidents. The LPCS system is a single train system which provides low pressure coolant to the reactor vessel during accidents. LPCI has three trains $\mathrm{A}, \mathrm{B}$, and $\mathrm{C}$, each with the capability of providing low pressure coolant to the reactor during accidents.
Trains A and B each have two heat exchangers which can be used to provide decay heat removal at shutdown or RHR during accidents. The results of the risk impact for single and multiple train maintenance on the ECCS are discussed in Sections 3.4.1 through 3.4.4, and Sections 3.4.1.1, 3.4.2.1, 3.4.3.1, 3.4.4.1 provide insights on single and multiple ECCS maintenance activities.

\subsubsection{Single Train Maintenance on HPCS}

The results of the $I_{C D F}$ for HPCS, in all seven POSs, are listed in Appendix $\mathrm{C}$ and are shown graphically in Figure 18. The results indicate that there is a medium $\mathrm{I}_{\mathrm{CDF}}$ during the first few days of low power and hot shutdown

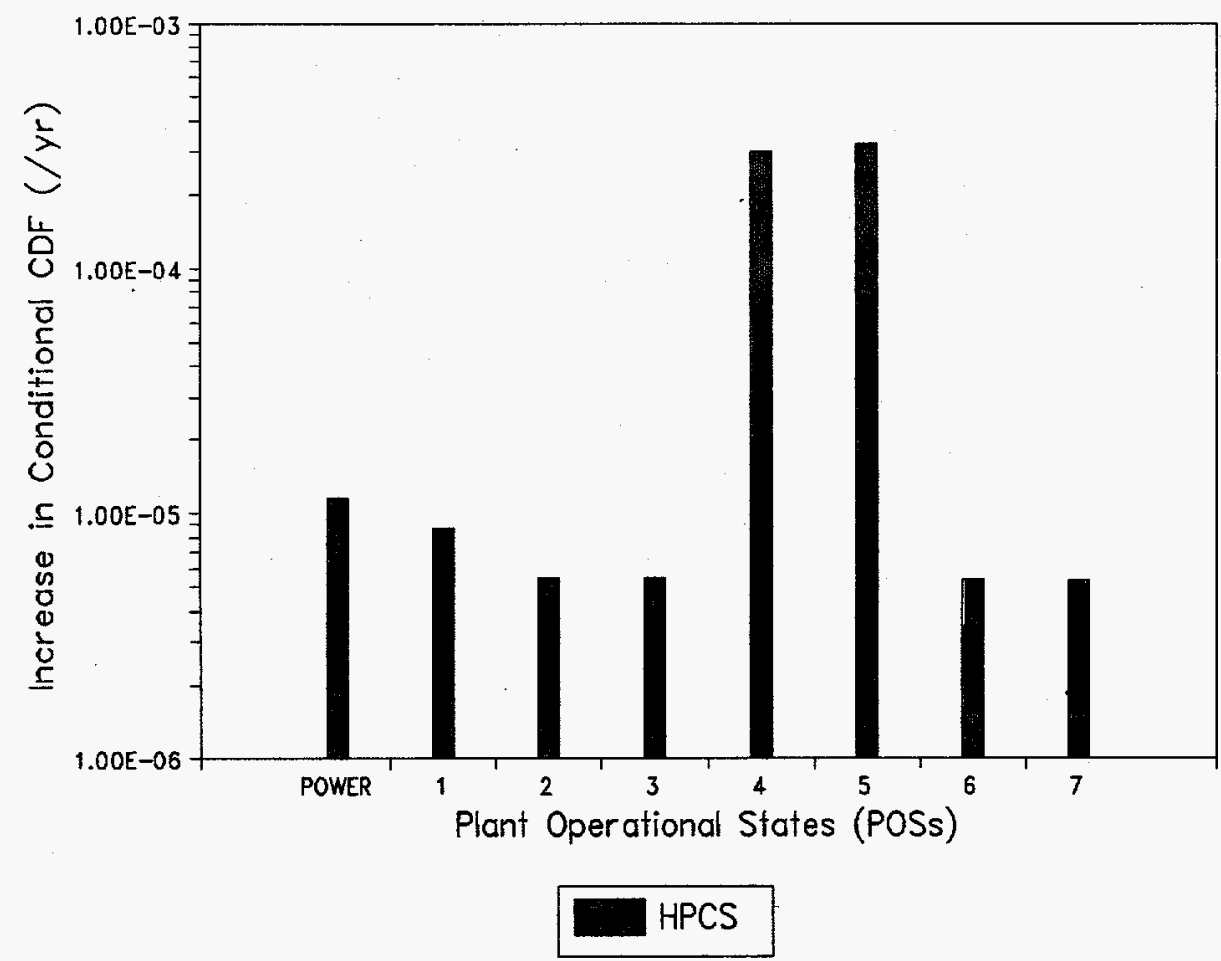

Figure 18. Increase in Conditional Core Damage Frequency for HPCS Maintenance 
(POSs 1, 2, and 3) which is comparable to that at power. However, the $I_{C D F}$ values are high in POSs 4 and 5 . These higher values are related to the fact that, unlike at power where HPCS is primarily used for ECCS injection, in POSs 4 and 5, HPCS additionally provides ECCS level control, water solid closed loop core cooling, and high pressure steaming operations. Also, in POS 4 and 5, the decay heat is still significant, the coolant inventory is limited, and the frequency of one of the most important accidents requiring high pressure coolant inventory, LOSP (T1), increases from $0.11 / y r$ to $0.13 / y r$. In POSs 6 and 7 , the $I_{C D F}$ is not substantially reduced compared to power.

The results of the HPCS $I_{C D P s}$ and $A_{I C D F}$ are listed in Appendix $C$. The $I_{\mathrm{CDPs}}$ are based on AOTs of 14 days with the plant operating at power and low power. An AOT of 24 hours is used when the plant is operating in hot shutdown, and an AOT equivalent to the time spent in the POS is used for cases where an AOT is not specified at shutdown. The results indicate that HPCS $\mathrm{I}_{\mathrm{CDPs}}$ in POSs $0,1,2,3,4$, and 5 are in the small to medium range decreasing to negligible in refueling (POS 6 and POS 7). The $A_{I C D F}$ is based on a maintenance frequency of $0.88 / y r$. The results indicate that the $A_{I C D F}$ is low for power operations. A time profile of the change in core damage frequency for taking HPCS out for maintenance is shown in Figure 19.

\subsubsection{Insights on HPCS Maintenance}

The results discussed above provide the following insights regarding the scheduling of HPCS maintenance outages:

Even though the $I_{C D F}$ for HPCS out of service at power is in the medium range, preventative maintenance of up to 14 days can be scheduled at power operations with only a small increase in the probability of core damage. This AOT appears to be sufficient for HPCS maintenance at power.

When adhering to the current AOT of 14 days, excessive use of the LCOs to perform preventative maintenance on HPCS at power could significantly increase the $A_{1 C D F}$. For example, increasing the maintenance frequency, for HPCS, from $0.88 / \mathrm{yr}$ to $6 / y r$ changes the $A_{\text {ICDF }}$ to $2.69 \mathrm{E}-6 / \mathrm{yr}(\approx 37 \%$ of the baseline core damage frequency).

Based on the $\mathrm{I}_{\mathrm{CDF}}$, there seems to be no risk advantage in performing maintenance on HPCS during the early stages of shutdown.
- During refueling, the $I_{C D F}$ is only slightly decreased compared to power. Therefore, there appears to be only a small risk advantage to performing maintenance of longer than 14 days (e.g. HPCS overhauls) at refueling. Furthermore, for HPCS, the OTR in POS 7 that gives a core damage probability comparable to that at power $(4.48 \mathrm{E}-7)$ is approximately 30 days.

\subsubsection{Single Train Maintenance on LPCS}

The results of the $I_{\text {CDFs }}$ for LPCS, in all seven POSs, are listed in Appendix $\mathbf{C}$ and are shown graphically in Figure 20. The results indicate that there is a medium $\mathrm{I}_{\mathrm{CDF}}$ during the first few days of low power and hot shutdown (POSs 1 , 2 , and 3) which is comparable to that at power. However, the $I_{C D F}$ values are high in POSs 4 and 5 . These higher values are related to the fact that, unlike at power, where LPCS is primarily used for low pressure ECCS injection, in POSs 4 and 5, LPCS additionally provides ECCS level control, water solid closed loop core cooling, and low pressure steaming operations. In POS 6 and 7 , the $I_{C D F}$ is reduced (low) compared to power.

The results of the LPCS $I_{C D P s}$ and $A_{I C D F}$ are listed in Appendix $C$. The $I_{\text {CDPs }}$ are based on AOTs of 7 days with the plant operating at power and low power. An AOT of 24 hours is used when the plant is operating in hot shutdown, and an AOT equivalent to the time spent in the POS is used for cases where an AOT is not specified at shutdown. The results indicate that LPCS $I_{C D P s}$ in POSs 0 through 7 are in the small to negligible range. The $A_{I C D F}$ is based on a maintenance frequency of $0.88 / \mathrm{yr}$. The results indicate that the $A_{\text {ICDF }}$ is low for power operations. A time profile of the change in core damage frequency for taking LPCS out for maintenance is shown in Figure 21.

\subsubsection{Insights on LPCS Maintenance}

The results discussed above provide the following insights regarding the scheduling of LPCS maintenance outages:

Even though the $\mathrm{I}_{\mathrm{CDF}}$ for LPCS out of service at power is in the medium range, preventative maintenance of up to 7 days can be scheduled at power operations with only a small increase in the probability of core damage (3.45E-8). However, since the increase in the probability of core damage 
Risk Impact of Maintenance

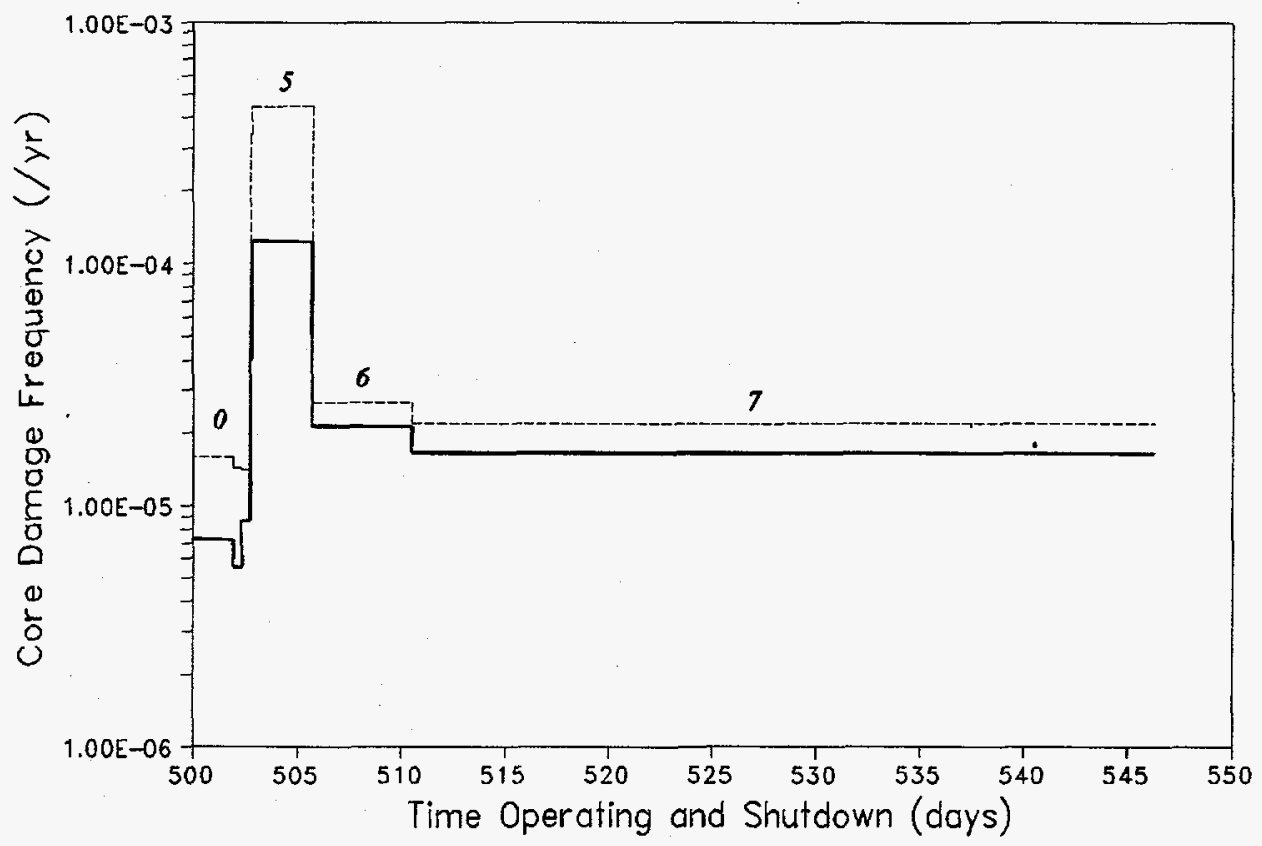

No Maintenance HPCS in Maintenance

Figure 19. Change in Core Damage Frequency for Taking HPCS Out for Maintenance During the Different POSs in an Operating Cycle 
Risk Impact of Maintenance

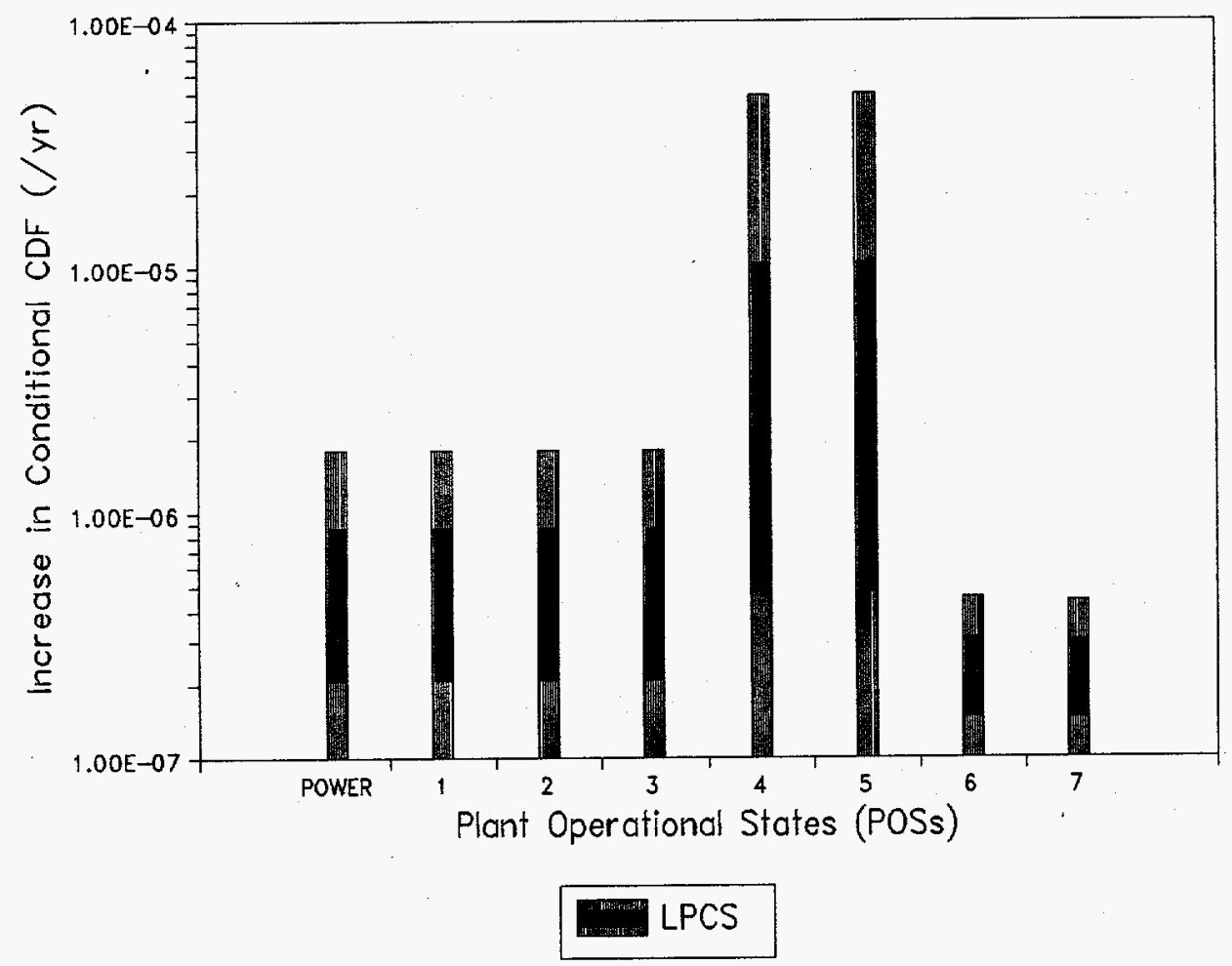

Figure 20. Increase in Conditional Core Damage Frequency for LPCS Maintenance 
Risk Impact of Maintenance

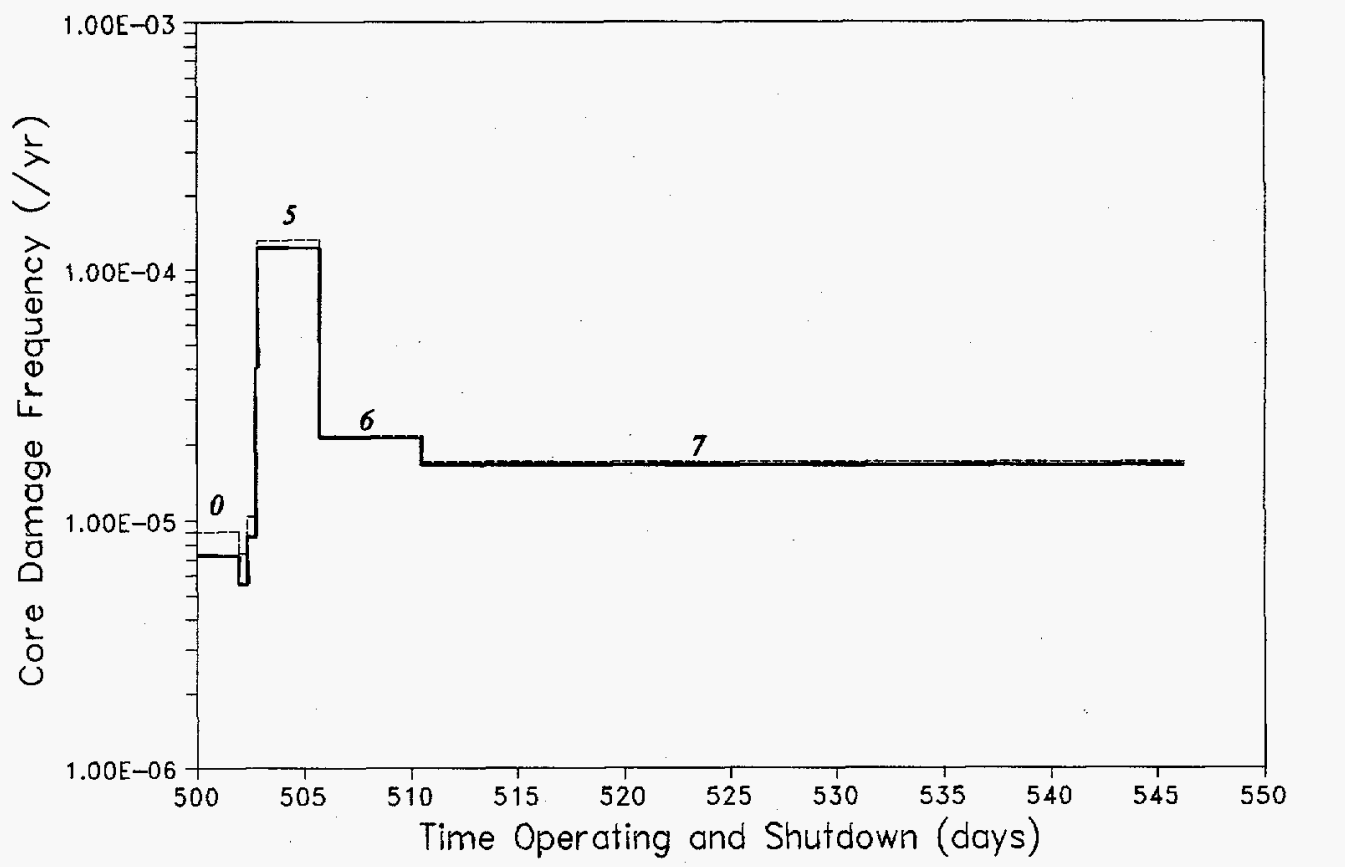

- Baseline $\quad$---- LPCS in M: intenance

Figure 21. Change in Core Damage Frequency for Taking LPCS Out for Maintenance During the Different POSs in an Operating Cycle 
is small, the possibility of extending the AOT could probably be considered without creating a substantial increase in the probability of core damage. For example, if the AOT is extended from 7 to 14 days, the core damage probability increases to $6.90 \mathrm{E}-8$ which is low. This extension could allow more time for diagnosis and proper repair and also create a uniform ECCS AOT for HPCS and LPCS at power.

- $\quad$ By limiting the AOT to 7 days, preventative maintenance on LPCS could probably be scheduled regularly at power without significantly increasing the $\mathrm{A}_{\mathrm{ICDF}}$. For example, increasing the maintenance frequency, for LPCS, from $0.88 / \mathrm{yr}$ to $6 / \mathrm{yr}$ changes the $\mathrm{A}_{\mathrm{ICDF}}$ to $2.07 \mathrm{E}-7 / \mathrm{yr}(\approx 3 \%$ of the baseline core damage frequency)

Based on the $\mathrm{I}_{\mathrm{CDF}}$, there appears to be no advantage in performing maintenance on LPCS during the early stages of shutdown. However, the $\mathrm{I}_{\mathrm{CDF}}$ at refueling is significantly reduced as compared to power. Consequently, preventative maintenance of longer than 7 days (e.g., LPCS overhauls) should preferably be scheduled for refueling. Furthermore, for LPCS, the OTR in POS 7 that gives a core damage probability comparable to that at power $(3.45 \mathrm{E}-8)$ is approximately 29 days. This indicates that LPCS could possibly be out for a longer duration if maintained at refueling as opposed to power.

\subsubsection{Single Train Maintenance on LPCI A/ RHR A}

The results of the $\mathrm{I}_{\mathrm{CDFs}}$ for LPCI $\mathrm{A}$, in all seven POSs, are listed in Appendix $\mathrm{C}$ and are shown graphically in Figure 22. The results indicate that there is a low $\mathrm{I}_{\mathrm{CDF}}$ during low power and the first few days of hot shutdown (POSs 1, 2 and 3 ) which is comparable to that at power. However, the $I_{\text {CDFs }}$ with the plant operating in POS 4 through 7 increase to the medium range. This is due to the fact that during shutdown, the plant is dependent on RHR for normal means of core cooling. Hence, at shutdown, loss of RHR is an initiating event. Furthermore, RHR is aligned for SDC at shutdown and thus must be manually aligned and initiated (i.e., RHR is dependent on an operator action to be successful). Whereas, at power, LPCI/RHR is used primarily for LPCI injection during an accident and is normally in standby mode. Furthermore, LPCI/RHR will auto-start given appropriate accident conditions (i.e., LPCI/RHR is not dependent on an operator action to function properly).

The results of the LPCI A/ RHR A I $\mathrm{I}_{\mathrm{CDPs}}$ and $\mathrm{A}_{\mathrm{ICDF}}$ are listed in Appendix $\mathrm{C}$. The $\mathrm{I}_{\mathrm{CDPs}}$ are based on AOTs of 7 days with the plant operating at power and low power. An AOT of 24 hours is used when the plant is operating in hot shutdown, and an AOT equivalent to the time spent in the POS is used for cases where an AOT is not specified at shutdown. The results indicate that LPCI $I_{C D P s}$ in POSs 1 through 7 are in the small to negligible range. The $A_{I C D F}$ is based on a maintenance frequency of $0.88 / \mathrm{yr}$. The results indicate that the $A_{I C D F}$ is negligible for power operations. $A$ time profile of the change in core damage frequency for taking a single train of LPCI out for maintenance is shown in Figure 23.

\subsubsection{Insights on LPCI A/ RHR A Maintenance}

The results discussed above provide the following insights regarding the scheduling of LPCI A/ RHR A maintenance outages:

Even though the $I_{C D F}$ for LPCI A out of service at power is in the low range, preventative maintenance of up to 7 days can be scheduled at power operations with only a negligible increase in the probability of core damage (8.42E-9). However, since the increase in the probability of core damage is negligible, the possibility of extending the AOT could be considered without creating a substantial increase in core damage probability. For example, if the AOT is extended from 7 to 14 days, the core damage probability increases to $1.68 \mathrm{E}-8$ which is low. This extension could allow more time for diagnosis and proper repair and give a common ECCS AOT for HPCS, LPCS, and LPCI A. 
Risk Impact of Maintenance

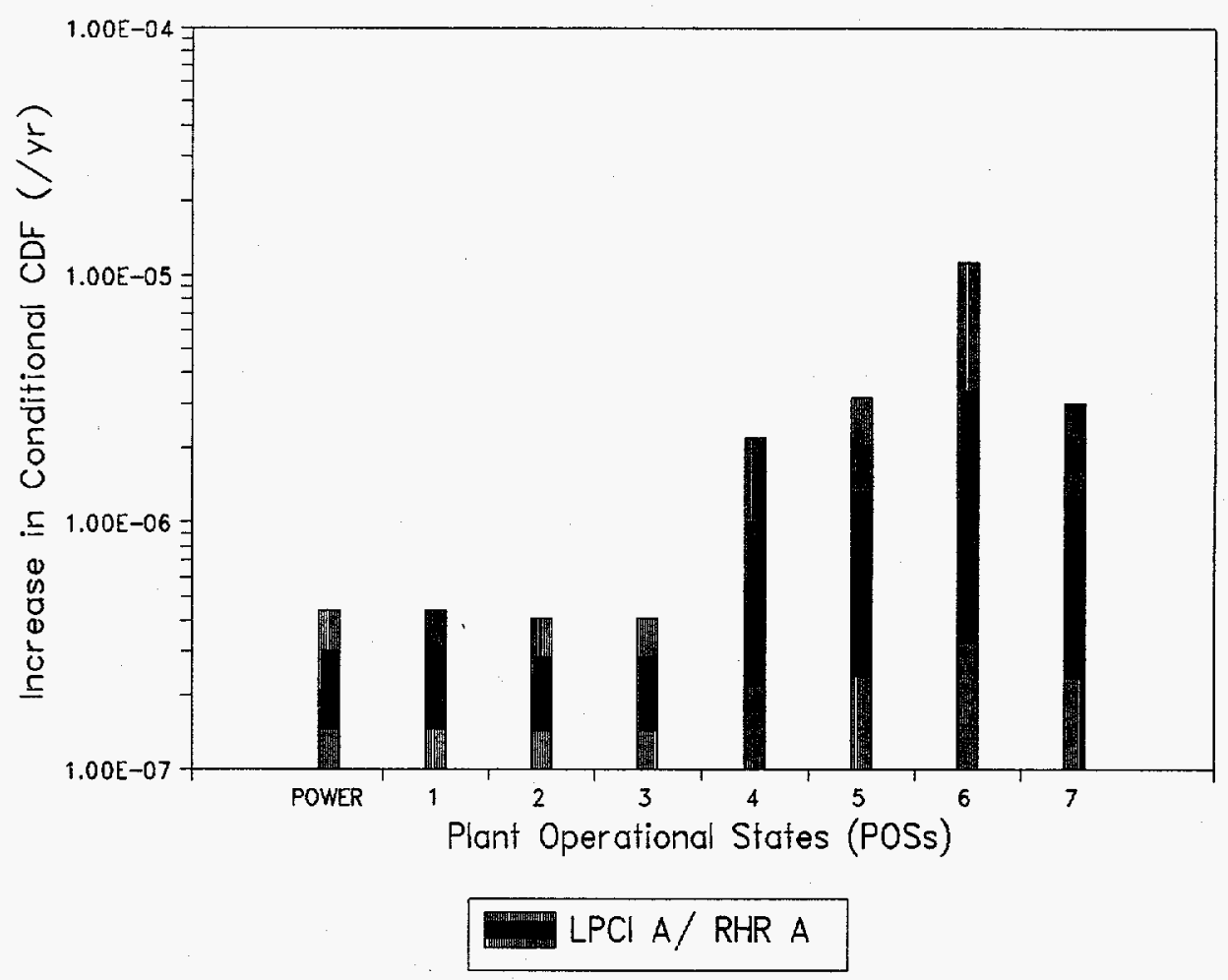

Figure 22. Increase in Conditional Core Damage Frequency for LPCI A/RHR A Maintenance 


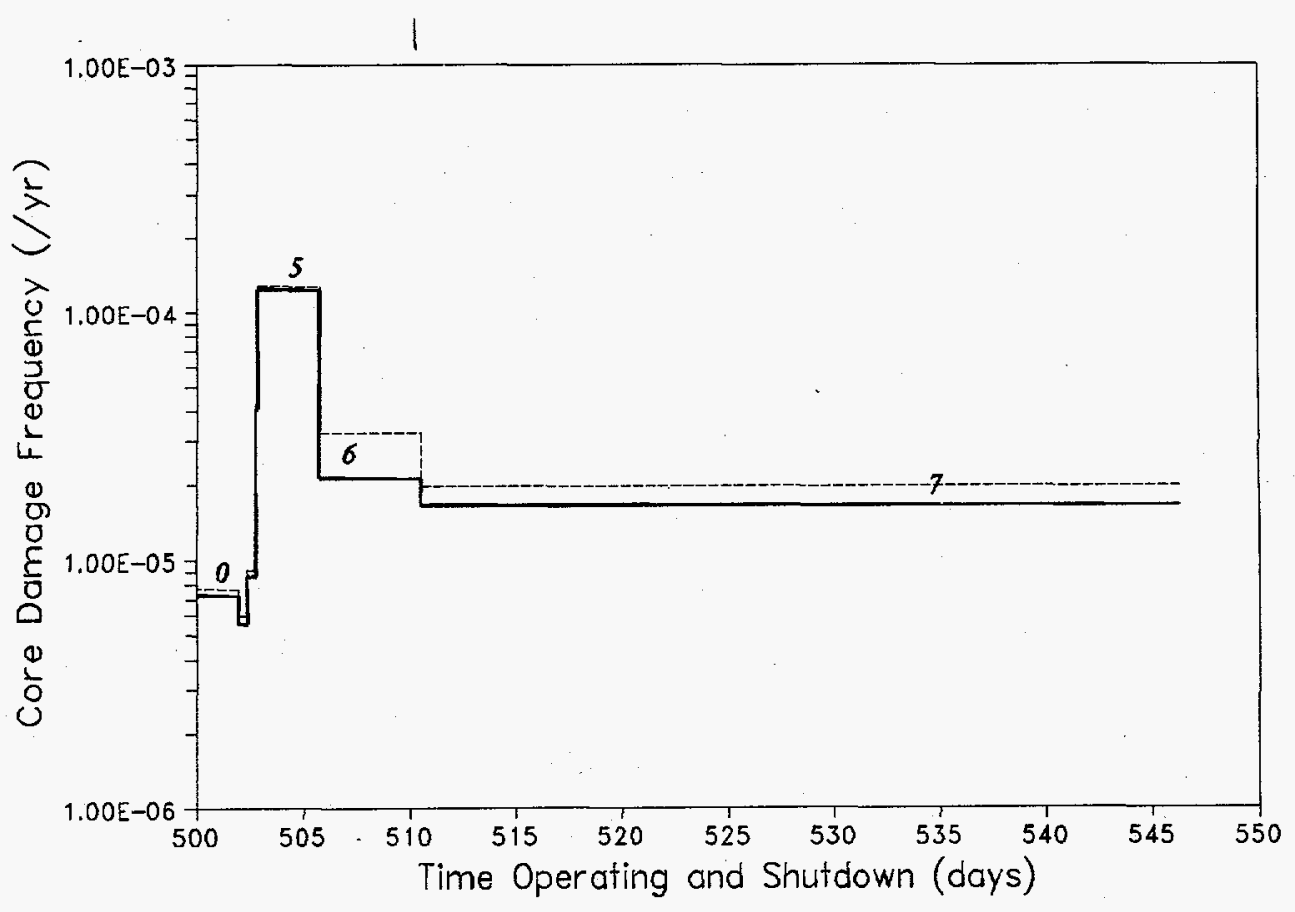

$$
\text { - No Maintenance }
$$

Figure 23. Change in Core Damage Frequency for Taking LPCI A Out for Maintenance During the Different POSs in an Operating Cycle 
By limiting the AOT to 7 days, preventative maintenance on LPCI A could probably be scheduled regularly at power without significantly increasing the $A_{I C D F}$. For example, increasing the maintenance frequency, for LPCS, from $0.88 / \mathrm{yr}$ to $6 / \mathrm{yr}$ changes the $A_{I C D F}$ to $5.02 \mathrm{E}-8 / \mathrm{yr}(<1 \%$ of the baseline core damage frequency).

Based on the $I_{C D F}$, there appears to be no risk advantage in performing maintenance on LPCI A during shutdown and refueling as opposed to power.
Furthermore, for LPCI A, the OTR in POS 7 that gives a core damage probability comparable to that at power (8.42E-9) is approximately 1 day.

\subsubsection{Multiple Train Maintenance on RHR A and $\mathrm{C}$}

The results of the $I_{\mathrm{CDFs}}$ for $\mathrm{LPCl} A$ and $C$, in all seven POSs, are listed in Appendix C and are shown graphically in Figure 24. The results indicate that there is a low $\mathrm{I}_{\mathrm{CDF}}$

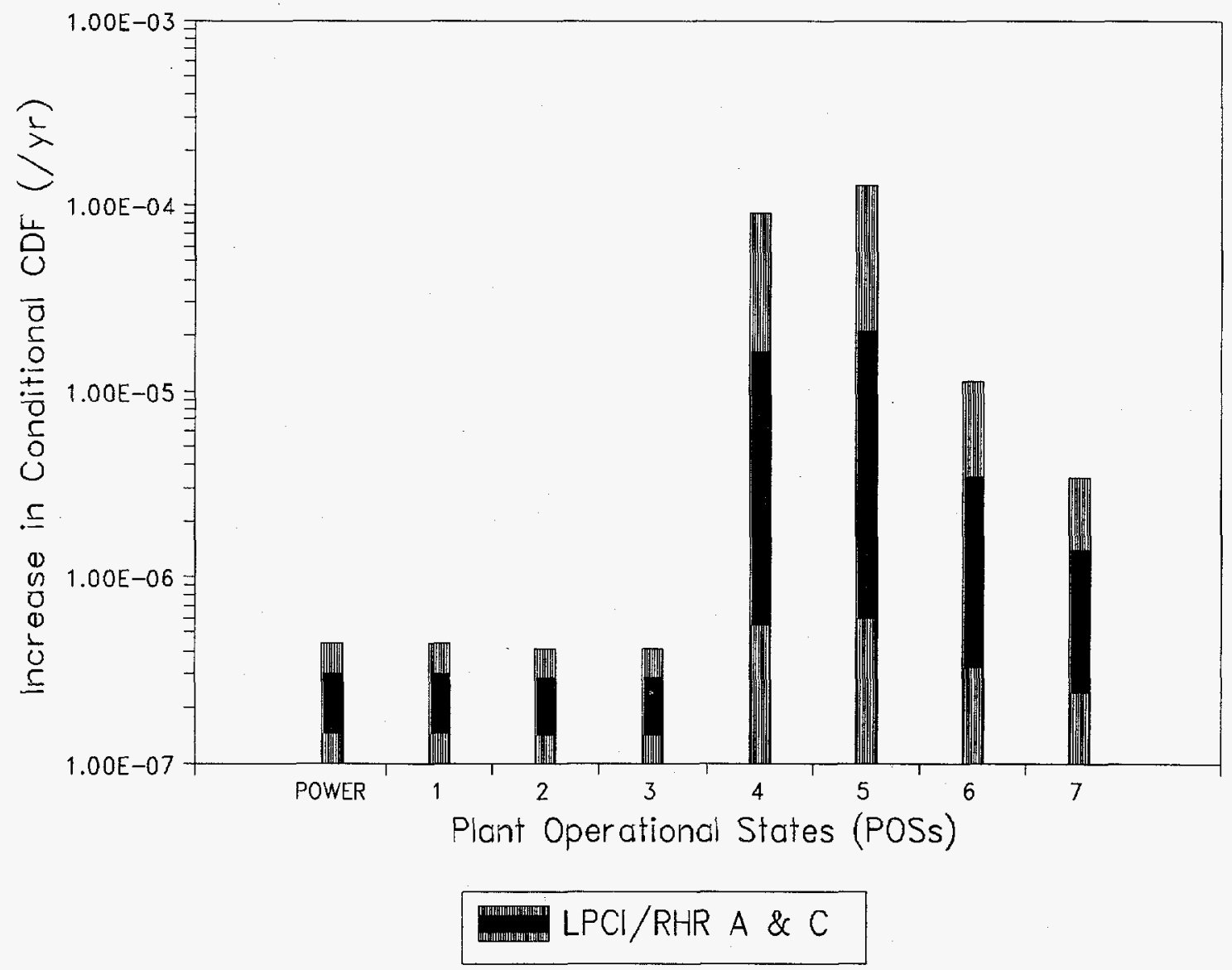

Figure 24. Increase in Conditional Core Damage Frequency for Multiple Train RHR Maintenance 
during low power and the first few days of hot shutdown (POSs 1,2 and 3) which is comparable to that at power. However, the $I_{C D F s}$ with the plant operating in POS 4 through 7 increase to the medium to high range. This is due to the fact that during shutdown, the plant is dependent on RHR for normal means of core cooling. Hence, at shutdown, loss of RHR is an initiating event. Furthermore, RHR is aligned for SDC at shutdown and thus must be manually aligned and initiated (i.e., RHR is dependent on an operator action to be successful). Whereas, at power, LPCI/RHR is used primarily for LPCI injection during an accident and is normally in standby mode. Furthermore, LPCI/RHR will auto-start given appropriate accident conditions (i.e., LPCI/RHR is not dependent on an operator action to function properly).

The RHR $A$ and $C I_{C D P s}$ and $A_{I C D F}$ are listed in Appendix $C$. These $I_{C D P s}$ are based on AOTs of 3 days with the plant operating at power and low power. An AOT of 24 hours is used when the plant is operating in hot shutdown, and an AOT equivalent to the time spent in the POS is used for cases where an AOT is not specified at shutdown.

The results indicate that LPCI A and C $I_{\text {CDPs }}$ in POSs 1 through 7 are in the small to negligible range. The $A_{1 C D F}$ is based on a maintenance frequency of $0.88 / y r$. The results indicate that the $A_{I C D F}$ is negligible for power operations.

\subsubsection{Insights on Multiple Train RHR A \& C Maintenance}

The results discussed above provide the following insights regarding the scheduling of LPCI A and C maintenance outages:

Even though the $\mathrm{I}_{\mathrm{CDF}}$ for LPCI $A$ and $\mathrm{C}$ out of service at power is in the low range, preventative maintenance of less than 3 days can be scheduled at power operations with only a negligible increase in the probability of core damage (3.61E-9). However, since the increase in the probability of core damage is negligible, the possibility of extending the AOT could probably be considered without creating a substantial increase in core damage probability. For example, if the AOT is extended from 3 to 9 days, the core damage probability increases to $1.0 \mathrm{E}-8$ which is low. This extension could allow more time for diagnosis and proper repair.

- The $\mathrm{I}_{\mathrm{CDF}}$ at refueling for maintenance on LPCI A and $C$ is increased compared to power. Thus, there appears to be no risk advantage over power to performing multiple LPCI maintenance at refueling. Furthermore, for LPCI A and C, the OTR in POS 7 that gives a core damage probability comparable to that at power (4.39E-7) is approximately 8 hours.

\subsection{Risk Impact of Reactor Core Isolation Cooling (RCIC) Maintenance Outage}

The RCIC system is a single train steam driven system which provides high pressure coolant injection to the reactor during accidents. The risk impact of maintaining RCIC is discussed in Section 3.5.1 below, and Section 3.5.1.1 provides insights on RCIC maintenance activities.

\subsubsection{Single Train Maintenance on the RCIC}

The results of the $\mathrm{I}_{\mathrm{CDFs}}$ for single train maintenance on RCIC, in POS 0, 1, 2, and 3, are listed in Appendix C and are shown graphically in Figure 25. The results indicate that there is a medium $I_{\mathrm{CDPs}}$ during low power and the first few days of hot shutdown which is comparable to that at power. However, since RCIC cannot be used in POS 4, 5, 6 , or 7 , the $\mathrm{I}_{\mathrm{CDFs}}$ during those POSs were not evaluated.

The results of the $\mathrm{I}_{\mathrm{CDPs}}$ and the $\mathrm{A}_{\mathrm{ICDF}}$ for RCIC are listed in Appendix C. The $\mathrm{I}_{\mathrm{CDPs}}$ are based on an AOT of 14 days at power, and low power, and an AOT of 24 hours in hot shutdown. The results indicate that the $\mathrm{I}_{\text {CPDs }}$ for $\mathrm{RCIC}$, in POS 1, 2, and 3, are small and are comparable to that at power. The $A_{I C D F}$ is based on a maintenance frequency of 2.64/yr. The results indicate that the $A_{I C D F}$ is medium for power operations.

\subsubsection{Insights on RCIC Maintenance}

The results discussed above provide the following insights regarding the scheduling of RCIC maintenance outage:

Even though the $\mathrm{I}_{\mathrm{CDF}}$ for $\mathrm{RCIC}$ out of service at power is in the medium range, preventative maintenance on RCIC can be scheduled at power for durations of up to $\mathbf{1 4}$ days resulting in a low probability of core damage (5.99E-7).

When adhering to the current AOT of 14 days, excessive use of the LCOS to perform preventative 
Risk Impact of Maintenance

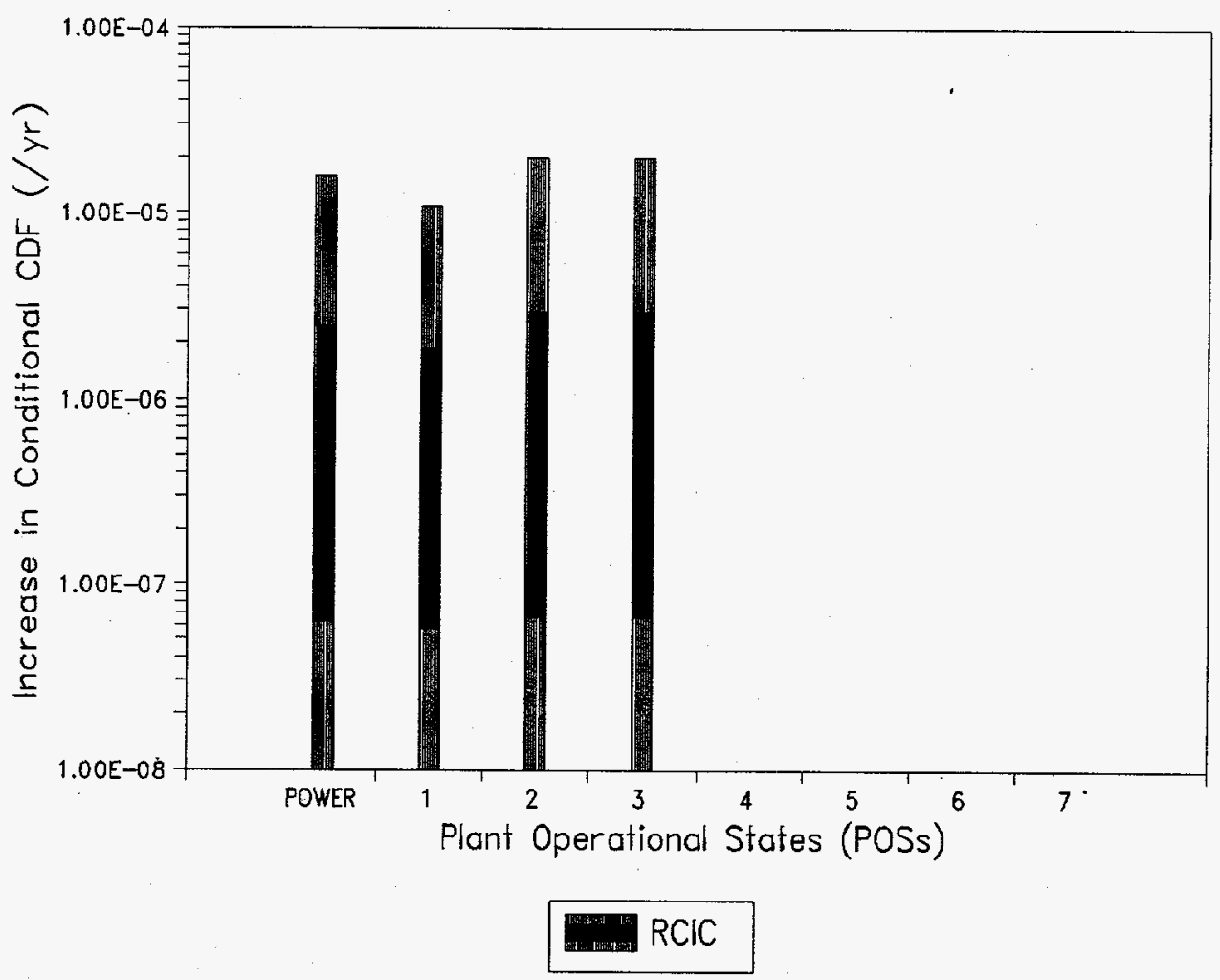

Figure 25. Increase in Conditional Core Damage Frequency for RCIC Maintenance 
maintenance on RCIC at power could significantly increase the $A_{I C D F}$. For example, increasing the maintenance frequency, for RCIC, from $2.64 / \mathrm{yr}$ to $6 / \mathrm{yr}$ changes the $\mathrm{A}_{\mathrm{ICDF}}$ to $3.60 \mathrm{E}-6 / \mathrm{yr}$ ( $\approx 49 \%$ of the baseline core damage frequency).

- Based on the $\mathrm{I}_{\mathrm{CDF}}$, there appears to be no risk advantage in performing maintenance on RCIC during the early stages of hot shutdown.

- Since RCIC is not available or required to operate in POSs 4 through 7, preventative maintenance on the system could possibly be performed in these plant conditions with negligible plant risk.

\subsection{Risk Impact of Suppression Pool (ISSP) Drainage and Safety Relief Valves (SRVs) Maintenance Outage}

For this analysis, the risk impact of draining the suppression pool for maintenance and the impact of performing maintenance on all SRVs were evaluated. The suppression pool provides the suction source for all ECCS and also acts as a heat sink for DHR. It is not expected that the suppression pool would ever be completely drained for maintenance even though the TSs permit drainage in POSs $4,5,6$, and 7 . However, this analysis was done to determine the vulnerability of the plant should it be drained. Normally during shutdown conditions, at least 2 SRVs are required to be operational. The SRVs are required for water solid closed loop core cooling, containment flooding, and steaming the core at low and high pressure. Without the SRVs none of these core cooling functions could be performed. In POSs 6 and 7, the reactor vessel head is removed and so the SRVs are not needed. The risk impact of draining the suppression pool and maintaining the SRVs are discussed in Section 3.6.1 below, and Section 3.6.1.1 provides insights on suppression pool drainage and SRV maintenance activities.

\subsubsection{ISSP and Maintenance on all SRVs}

The results of the suppression pool (ISSP) drainage and SRV $I_{\mathrm{CDFs}}$ are listed in Appendix $\mathrm{C}$ and are shown graphically in Figures 26 and 27. The results indicate that there is a high $\mathrm{I}_{\mathrm{CDF}}$ for ISSP in POSs 4 through 7 and a high $I_{C D F}$ for the SRVs in POSs 4 and 5. The results of the $I_{C D P s}$ for ISSP and SRVs are listed in Appendix C. The results indicate that $\mathrm{I}_{\mathrm{CDPs}}$ are in the medium to large range.

\subsubsection{Insights on ISSP and SRV Maintenance}

These results provide the following insights regarding the risk impact of draining the suppression pool for maintenance and the impact of performing maintenance on all SRVs:

Drainage of the suppression pool for maintenance results in a high increase in the $\mathrm{I}_{\mathrm{CDF}}$, and maintenance should preferably be performed in POS 7 where water level is high and the $\mathrm{I}_{\mathrm{CDF}}$ is lowest.

Simultaneous maintenance on all SRVs results in a high increase in the $\mathrm{I}_{\mathrm{CDF}}$ during shutdown. Since the SRVs are not needed when the vessel is open, maintenance should preferably be performed at refueling (POS 6 or 7). 
Risk Impact of Maintenance

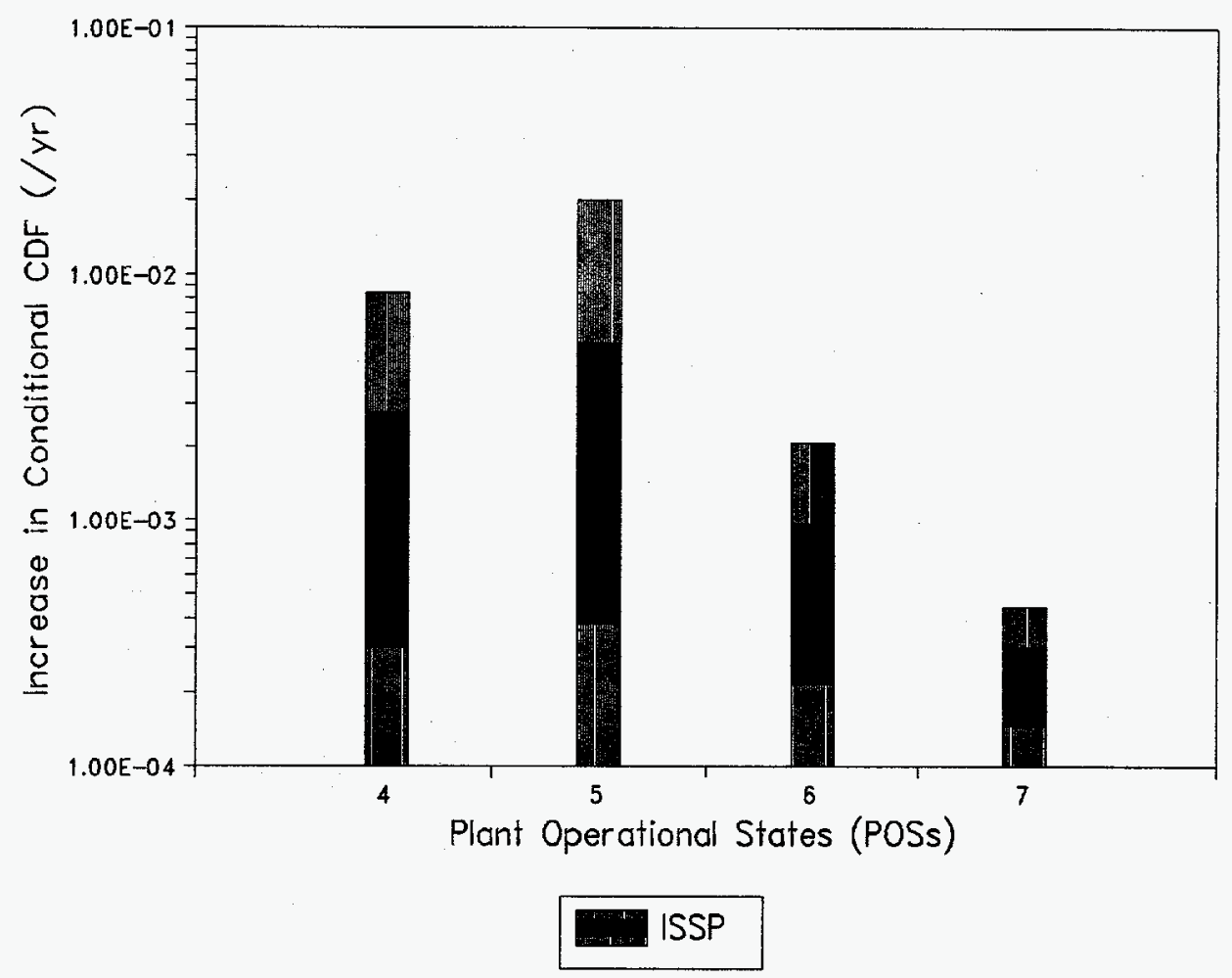

Figure 26. Increase in Conditional Core Damage Frequency for Suppression Pool Drainage 


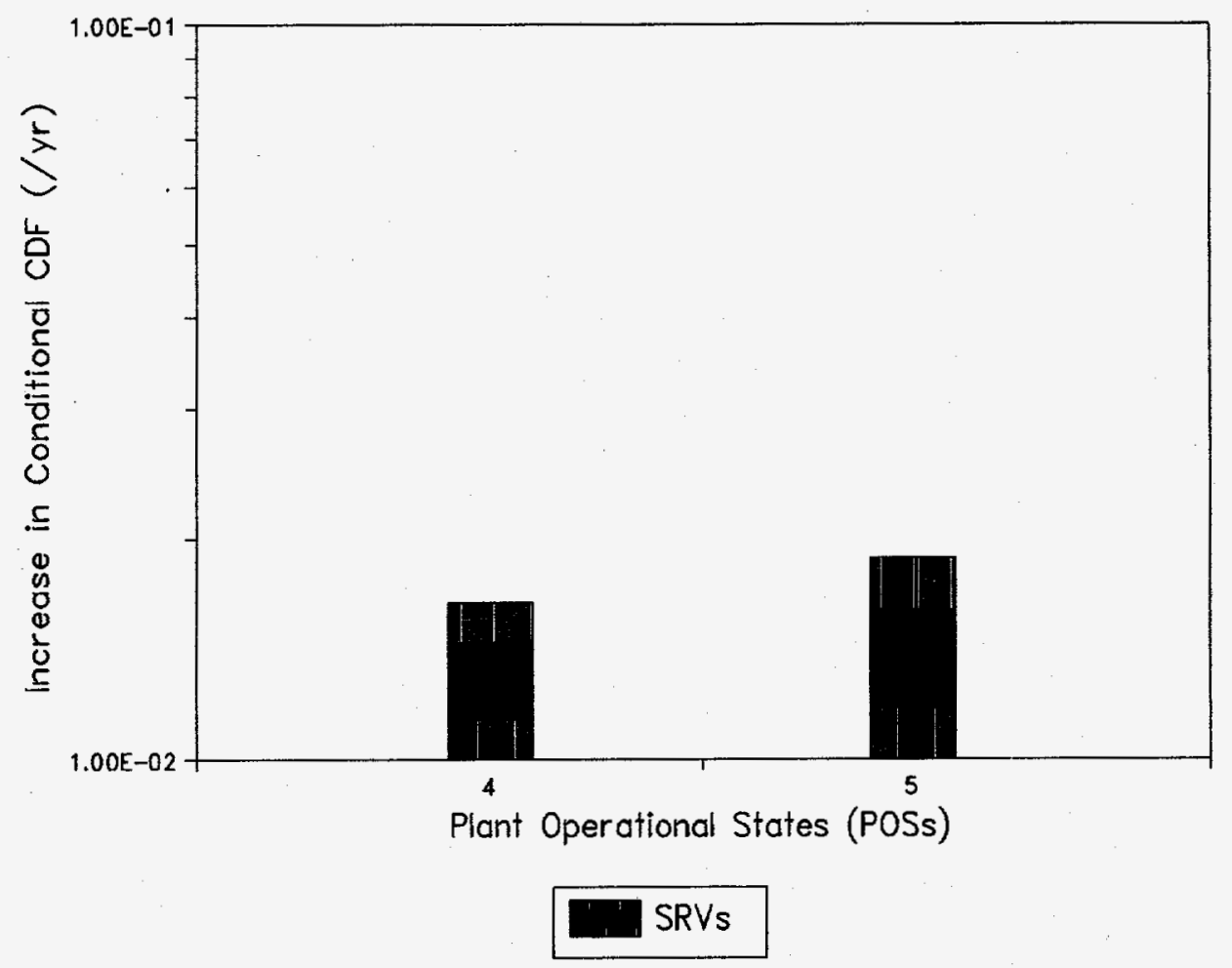

Figure 27. Increase in Conditional Core Damage Frequency for Simultaneous Maintenance on SRVs 


\subsection{Results of the Risk Impact of Surveillance Test Intervals}

This section of the report presents the results of the risk impact of surveillance tests on various plant systems during power operation, shutdown, and refueling. The systems include EDGs, SSW, BATTs, RCIC, ECCS, and RHR. Risk insights on scheduling and duration of various test activities are also presented. Note that the risk impact results for each surveillance test were determined using generic failure data. More accurate results could possibly be obtained by using plant specific data.

\subsection{Risk Impact of Testing at Power}

The risk benefit of performing various testing activities at power are listed in Table 4 and are shown graphically in Figure 28. Sections 4.1.1 through 4.1.4 describe the risk benefits of individual tests performed at power, discuss the sensitivity of the tests to STI changes, and identify potential risk improvements that could occur if the scope or timing (not just the frequency) of a surveillance was changed.

\subsubsection{Emergency Diesel Generator (EDG) Surveillance.}

During power operations, the EDGs are manually started and loaded for at least one hour each month. During this test, the dedicated SSW system used for EDG jacket cooling is also required to start. Therefore, the total risk benefit associated with the manual start test on an EDG includes the benefit of testing the dedicated SSW system and that of testing the EDG. For this evaluation, the Risk Benefits of the monthly surveillance for the EDGs are in the medium range, i.e 1.25E-6/yr for EDG III and 1.07E-6/yr for EDG I.

Since the Risk Benefit of monthly EDG testing is in the medium range, the current STI of a month appears to be reasonable and might not be a candidate for extension unless an alternative testing schedule can be determined which would increase the STI without altering plant risk. A significant percentage of the total Risk Benefit is contributed by the SSW portion of the test. Thus, by reducing the STI on SSW, the STI for an EDG could possibly be extended without changing the total Risk Benefit. For example, of the $1.07 \mathrm{E}-6 / \mathrm{yr}$ Risk Benefit associated with the testing of EDG 1, 3.82E-7/yr or $\approx 30 \%$ is due to the testing of SSW A. Hence, by testing SSW A every 15 days instead of every 31 days, the STI of the EDG could probably be extended to $\approx 37$ days without affecting the Risk Benefit.
Another approach might be to start, run, and stop SSW A (pre-test) before starting the EDG. This could detect any standby related failures in SSW A. In this case, the STI of the EDG could possibly be extended to $\approx 45$ days without affecting plant risk. If the STI of the EDG start test is doubled to 60 days, then the Risk Benefit becomes $2.50 \mathrm{E}-$ 6/yr for EDG III and 2.14E-6/yr for EDG I. The sensitivity of the Risk Benefit of the manual start tests on EDG III and EDG I to changes in the STI and the scope of the tests discussed above are shown in Figure 29. Any extension of the STI for the EDG start test could possibly reduce the burden of EDG surveillance at the plant and lessen the impact of wear on the EDGs due to frequent testing. Note that when considering any extensions to the STIs on the EDGs, care must be taken to adhere to the manufacturers recommendations on surveillance.

\subsubsection{Emergency Core Cooling System (ECCS) Surveillance}

The ECCS is periodically tested during power operations. Tests include quarterly flow tests on the pumps and valves and quarterly stroke tests on specific valves. During the quarterly flow tests, the dedicated SSW used for pump and room cooling is also required to start. Therefore, the total Risk Benefit of the ECCS flow test includes the benefit of testing the dedicated SSW and that of testing ECCS. For this evaluation, consideration was given to the fact that parts of the SSW, including the pump and certain inlet and outlet valves, are tested monthly during the monthly EDG start test. Based on this, the Risk Benefit of the quarterly flow test on the ECCS is in the low range.

A significant percentage of the total Risk Benefit of ECCS flow testing is due to SSW, thus, by modifying the STI on SSW, the STI for the ECCS surveillance could be considered for extension without affecting plant risk. For example, of the $6.82 \mathrm{E}-7 / \mathrm{yr}$ Risk Benefit associated with the HPCS flow test, $1.38 \mathrm{E}-7 / \mathrm{yr}$ or $\approx 20 \%$ is due to SSW C. Therefore, by starting SSW C every 15 days instead of every 31 days, the HPCS flow test could be possibly be extended to $\approx 3.4$ months without affecting the plant risk. Another possible approach would be to pre-test SSW C before performing the HPCS flow test to detect any standby related failures in SSW C. In this case, the STI of HPCS could possibly be extended to $\approx 4$ months without affecting the plant risk. A third approach could be to perform monthly stroke tests on the specific valves in the ECCS flow 
Table 4. Risk Impact of Tests Performed at Power

\begin{tabular}{|c|c|c|c|c|}
\hline Rank & System & Test Type & $\begin{array}{l}\text { STI } \\
\text { (days) }\end{array}$ & $\begin{array}{c}\text { Risk } \\
\text { Impact } \\
\text { (/yr) }\end{array}$ \\
\hline 1 & EDG III & Manual Start Test & 31 & 1.25E-6 \\
\hline 2 & EDG I & Manual Start Test & 31 & $1.07 \mathrm{E}-6$ \\
\hline 3 & RCIC & Flow Test & 92 & $1.07 \mathrm{E}-6$ \\
\hline 4 & HPCS & Flow Test & 92 & $6.82 \mathrm{E}-7$ \\
\hline 5 & SSW A & Flow Test & 92 & $3.28 \mathrm{E}-7$ \\
\hline 6 & LPCS & Flow Test & 92 & $2.78 \mathrm{E}-7$ \\
\hline 7 & LPCI A & Flow Test & 92 & $2.74 \mathrm{E}-7$ \\
\hline 8 & $\begin{array}{c}\text { RCIC } \\
\text { MOV } 13\end{array}$ & Stroke & 92 & $1.78 \mathrm{E}-7$ \\
\hline 9 & $\begin{array}{l}\text { RCIC } \\
\text { MOV } 19\end{array}$ & Stroke & 92 & $1.78 \mathrm{E}-7$ \\
\hline 10 & $\begin{array}{l}\text { RCIC } \\
\text { MOV } 22\end{array}$ & Stroke & 92 & $1.78 \mathrm{E}-7$ \\
\hline 11 & BATT-I & $\begin{array}{l}\text { Connected Cell specific gravity, float voltage, and temperature } \\
\text { measurement }\end{array}$ & 92 & $1.62 \mathrm{E}-7$ \\
\hline 12 & SSW C & Flow Test & 92 & $1.38 \mathrm{E}-7$ \\
\hline 13 & $\begin{array}{l}\text { HPCS } \\
\text { MOV } 4\end{array}$ & Stroke & 92 & $9.07 \mathrm{E}-8$ \\
\hline 14 & BATT-III & $\begin{array}{c}\text { Connected cell specific gravity, float voltage, and temperature } \\
\text { measurement }\end{array}$ & 92 & $5.48 \mathrm{E}-8$ \\
\hline 15 & $\begin{array}{l}\text { LPCS } \\
\text { MOV } 5\end{array}$ & Stroke & 92 & $1.64 \mathrm{E}-8$ \\
\hline 16 & BATT I & $\begin{array}{c}\text { Pilot cell specific gravity, float voltage, and temperature } \\
\text { measurement }\end{array}$ & 7 & $1.22 \mathrm{E}-8$ \\
\hline 17 & $\begin{array}{c}\text { LPCI } \\
\text { MOV } \\
42 \mathrm{~A}\end{array}$ & Stroke & 92 & $9.27 \mathrm{E}-9$ \\
\hline 18 & BATT III & $\begin{array}{c}\text { Pilot cell specific gravity, float voltage, and temperature } \\
\text { measurement }\end{array}$ & 7 & 4.39E-9 \\
\hline
\end{tabular}


Risk Impact of Surveillance Tests

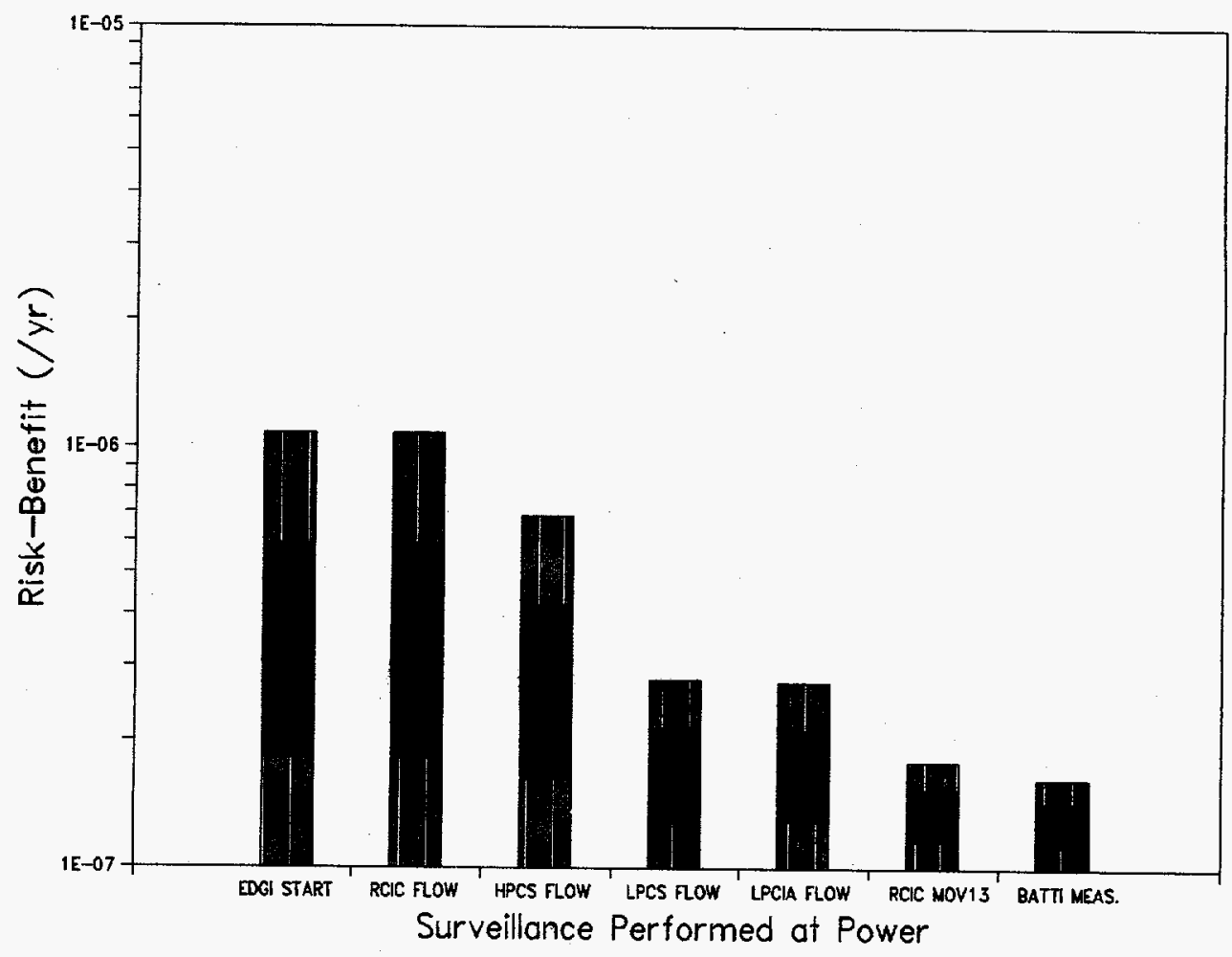

Figure 28. Risk Benefit of Surveillance Tests Performed at Power 
Risk Impact of Surveillance Tests

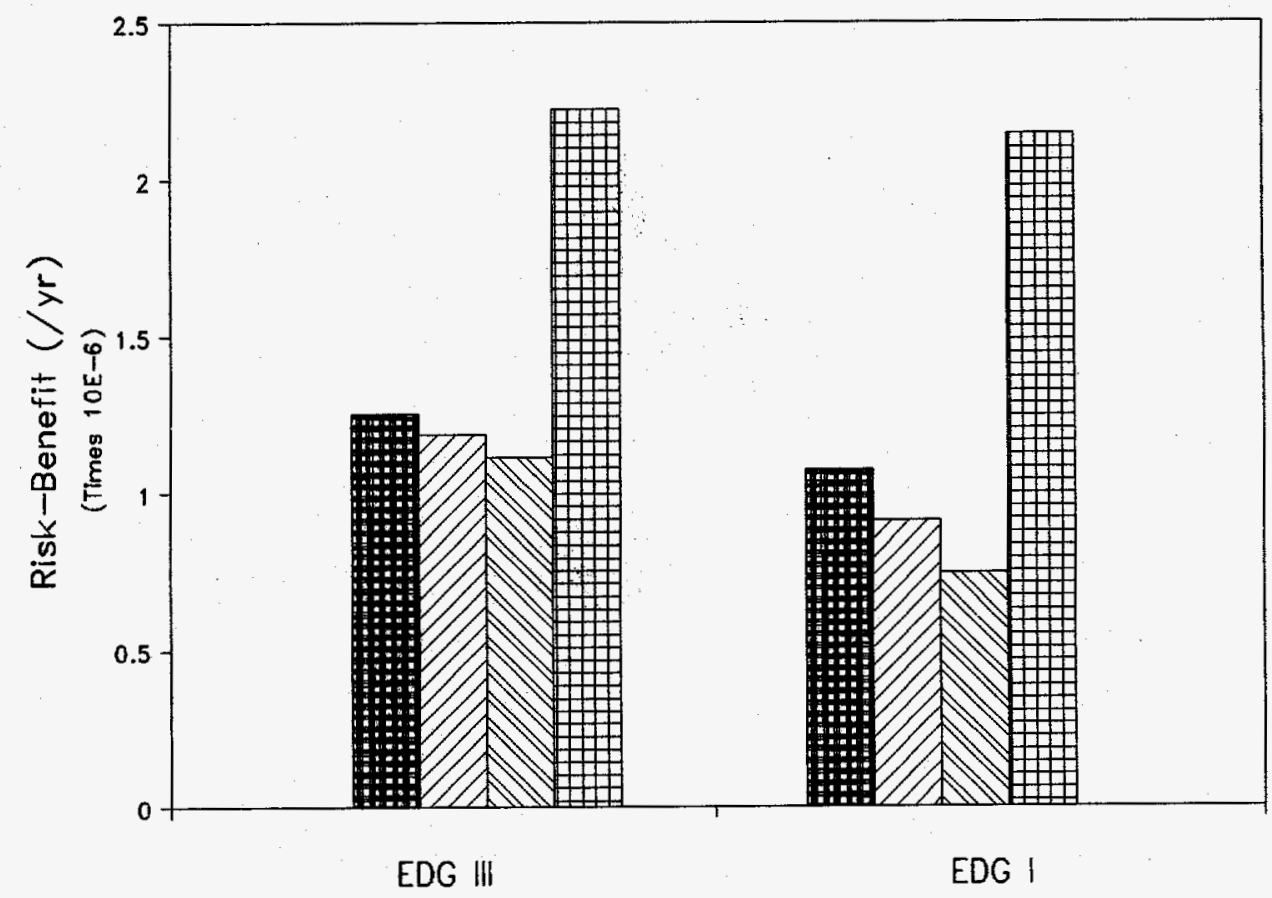

19: EDG manual start $D Z D S S W$ STI = 15 days $\triangle$ SSW Pre-fested $\quad$ STI Doubled

Figure 29. Risk Benefit Sensitivities to Different EDG Surveillance Test Arrangements 
path being tested. For example, if all the valves in the HPCS flow path are stroked monthly, the STI of the ECCS flow test could possibly be extended to $\approx 5.4$ months without affecting the plant risk. Any extension of the STIs on the ECCS pumps could possibly reduce the burden of the surveillance at the plant and lessen the impact of wear on ECCS pumps due to frequent testing.

Valves, such as LPCI motor-operated valve (MOV) $42 \mathrm{~A}$ or LPCS MOV 5, that are not tested during the quarterly flow tests are stroked quarterly. For these valves, the Risk Benefits of the quarterly stroke tests are in the low to negligible range and thus might be candidates for STI extension. For example, doubling the STI to 6 months for the stroke test on LPCI MOV 42 A would increase the risk impact to only $1.85 \mathrm{E}-8 / \mathrm{yr}$. However, when considering these extensions, thought might be given to the possibility of performing alternate forms of risk control activities such as quarterly visual inspection or other monitoring activities. The sensitivity of the Risk Benefit of the ECCS flow tests to changes in the STI and the scope of the tests discussed above are shown in Figure 30.

\subsubsection{Standby Service Water (SSW) Surveillance}

The SSW is periodically tested during power operations. Tests include quarterly flow tests on the pumps and valves and quarterly stroke tests on specific MOV valves. In addition, the SSW is periodically tested as part of the EDG start test and other surveillances. Based on this, the Risk Benefit of the quarterly flow test on the SSW is in the low range, i.e., $3.28 \mathrm{E}-7 / \mathrm{yr}$ and $1.38 \mathrm{E}-7$ for SSW A and SSW C flow tests, respectively.

\subsubsection{Battery (BATT) Surveillances}

During power operations, unit batteries' specific gravity, pilot cell voltage, and temperature are measured weekly and compared to a set of limits prescribed in the TSs. Quarterly, the voltage and specific gravity of each cell is measured and compared to another set of limits. The Risk Benefits of the weekly surveillance on BATT I and BATT III are low to negligible, that is $1.22 \mathrm{E}-8 / \mathrm{yr}$ for BATT I and 4.39E-9/yr for BATT III. Thus, these weekly battery surveillances appear to be possible candidates for STI extension. For example, by extending the weekly surveillance on BATT III to one month, the Risk Benefit becomes $1.76 \mathrm{E}-8 / \mathrm{yr}$ which is low.
The Risk Benefit of the quarterly surveillance on BATT I and BATT III are low, that is 1.62E-7/yr for BATT I and $5.48 \mathrm{E}-8 / \mathrm{yr}$ for BATT III. Thus, these quarterly battery surveillances appear to be possible candidates for STI extension. For example, doubling the quarterly surveillance on BATT I increases the Risk Benefit to 3.24E-7/yr which is low. However, when considering any extension, care should be taken to adhere to the manufacturers recommendations on surveillance.

\subsection{Risk Impact of Testing at Shutdown}

The Risk Benefit and the Risk Penalty due to Test Downtime for various tests performed at shutdown are listed in Appendix D.

\subsubsection{Emergency Diesel Generator (EDG) Surveillance}

During shutdown various surveillance tests are performed on the EDGs. For this analysis, the EDG 24 hour run/load test and the EDG 18 month functional test simulating a loss of offsite power (LOSP) were considered . The purpose of the 24 hour run/load test is to demonstrate the ability of the EDG to operate for 24 hours loaded to $\geq 5450 \mathrm{~kW}$ but not to exceed $5740 \mathrm{~kW}$. During the EDG 18 month functional test simulating a loss of power (LOP), the EDG auto starts and energizes the dedicated division BUS with the permanent connected loads. This surveillance usually follows the 24 hour run test with the primary purpose of checking the EDG actuation logic. Both of these tests are performed only at shutdown, but consideration was given to the fact that the EDGs are manually started on a monthly basis at power. The risk impact of performing these EDG surveillance are described below.

\subsubsection{EDG 24 Hour Load/Run Test}

The results of the risk impact of the EDG 24 hour load/run test are listed in Appendix D and shown in Figure 31. The results indicate that the Risk Benefit of the test is significant (e.g., 1.57E-5/yr for EDG I) in POS 5, 6, and 7 while the Risk Penalty due to Test Downtime is low to negligible. Since the Risk Benefit of the test is in the medium range, the current STI of 18 months appears to be reasonable and might not be a candidate for extension unless an alternative testing schedule could be determined which could increase the STI without altering plant risk. 
Risk Impact of Surveillance Tests

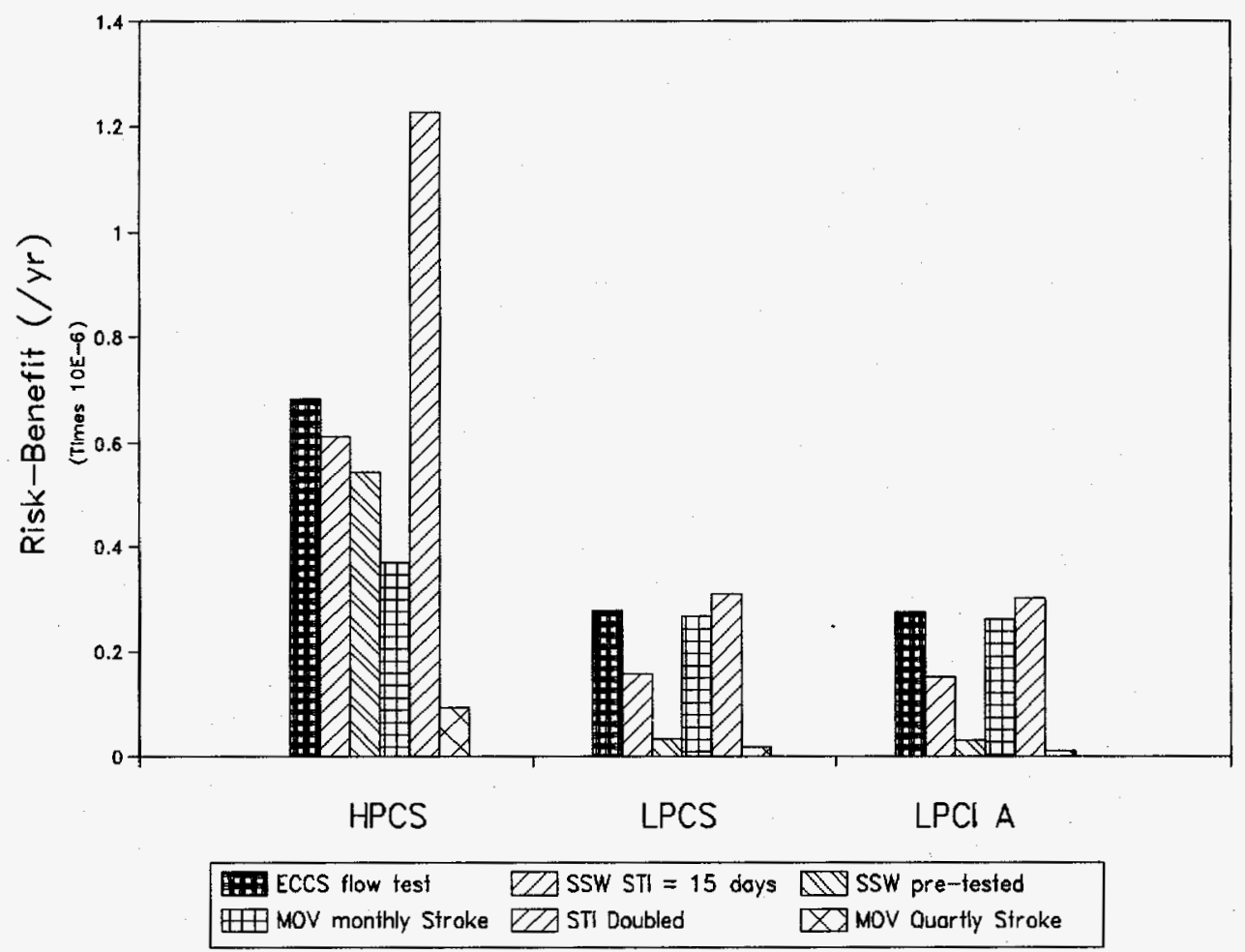

Figure 30. Risk Benefit Sensitivities to Different ECCS Surveillance Test Arrangements 
Risk Impact of Surveillance Tests

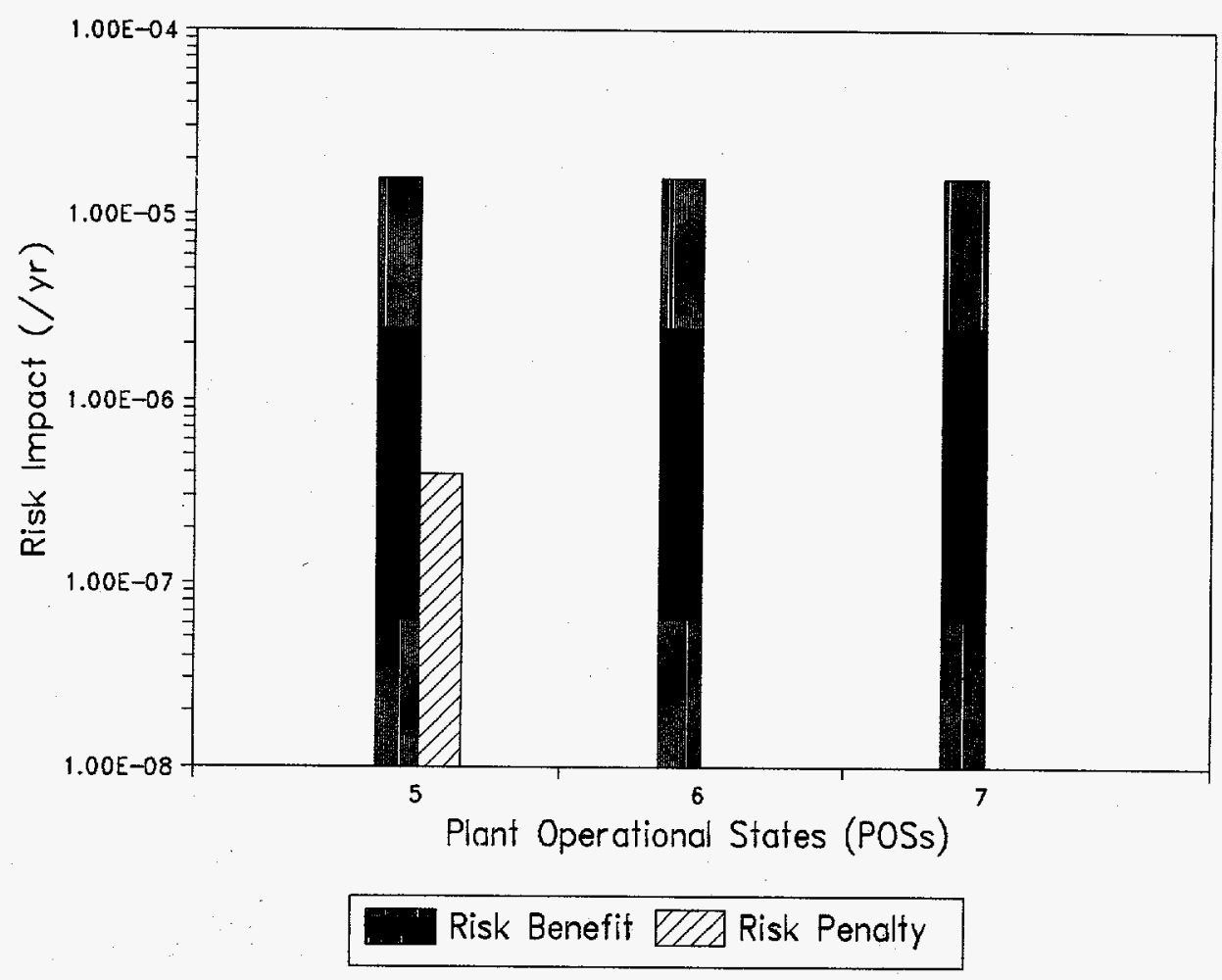

Figure 31. Risk Impact of the EDG 24 Hour Load/Run Test 


\subsubsection{EDG 18 Month Functional (LOP) Test}

The results of the risk impact of the EDG 18 month functional (LOP) test are listed in Appendix D and shown in Figure 32. The results indicate that the Risk Benefit of performing the EDG 18 month functional test at shutdown is low while the Risk Penalty due to Test Downtime is low to negligible.

\subsubsection{Battery (BATT) Surveillance}

During shutdown, emergency load discharge tests are performed on the batteries. These test are used to determine if the battery capacity is adequate to supply and maintain all of the actual emergency loads in an operable status. The results of the risk impact of the BATT 18 month emergency load discharge test are listed in Appendix D and shown in Figure 33. The results indicate that the Risk Benefit of performing the emergency load discharge tests on BATT I at shutdown is low while the Risk Penalty due to Test Downtime is low to negligible. Similar risk impacts were obtained for BATT III.

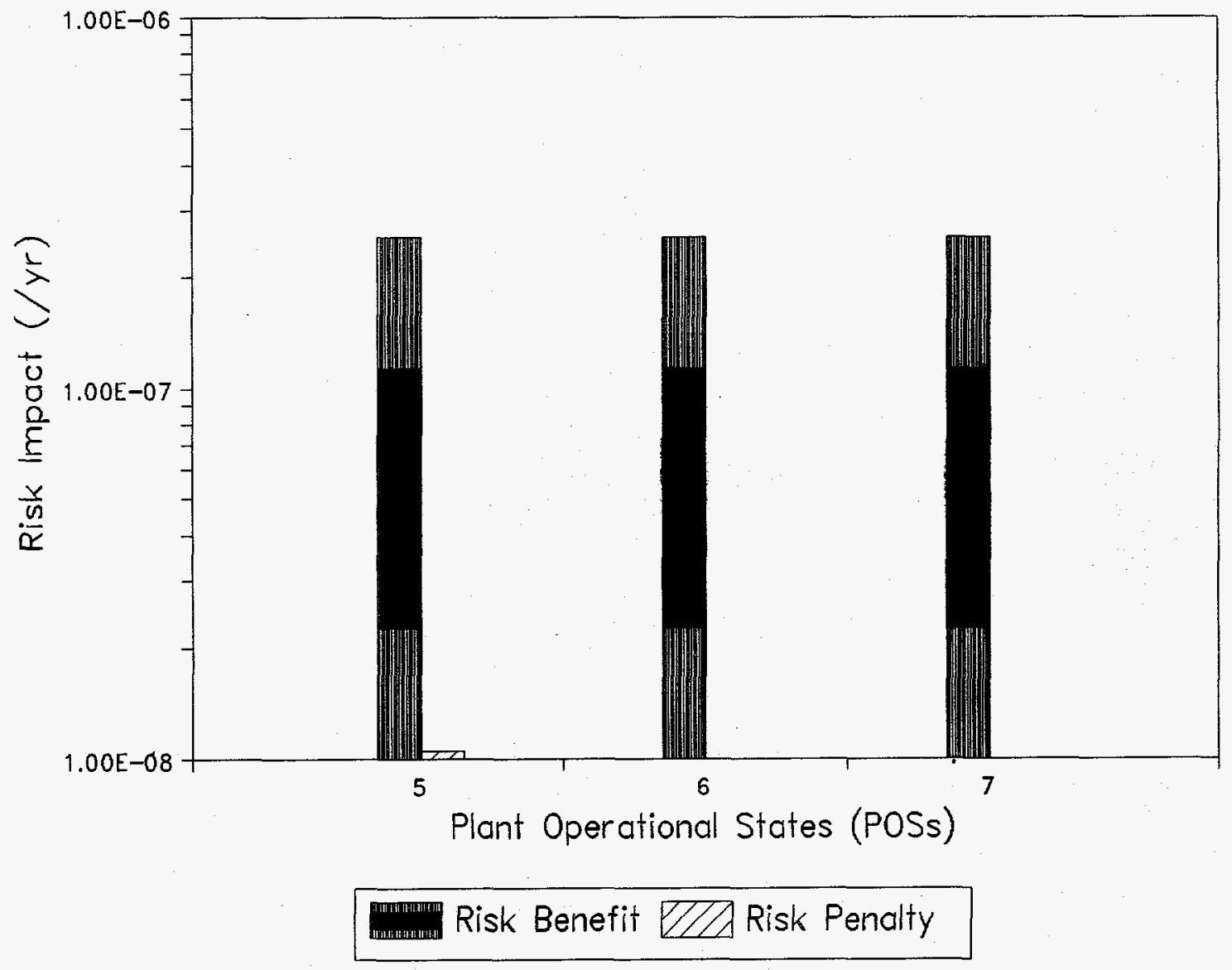

Figure 32. Risk Impact of the EDG 18 Month Functional (LOP) Test 
Risk Impact of Surveillance Tests

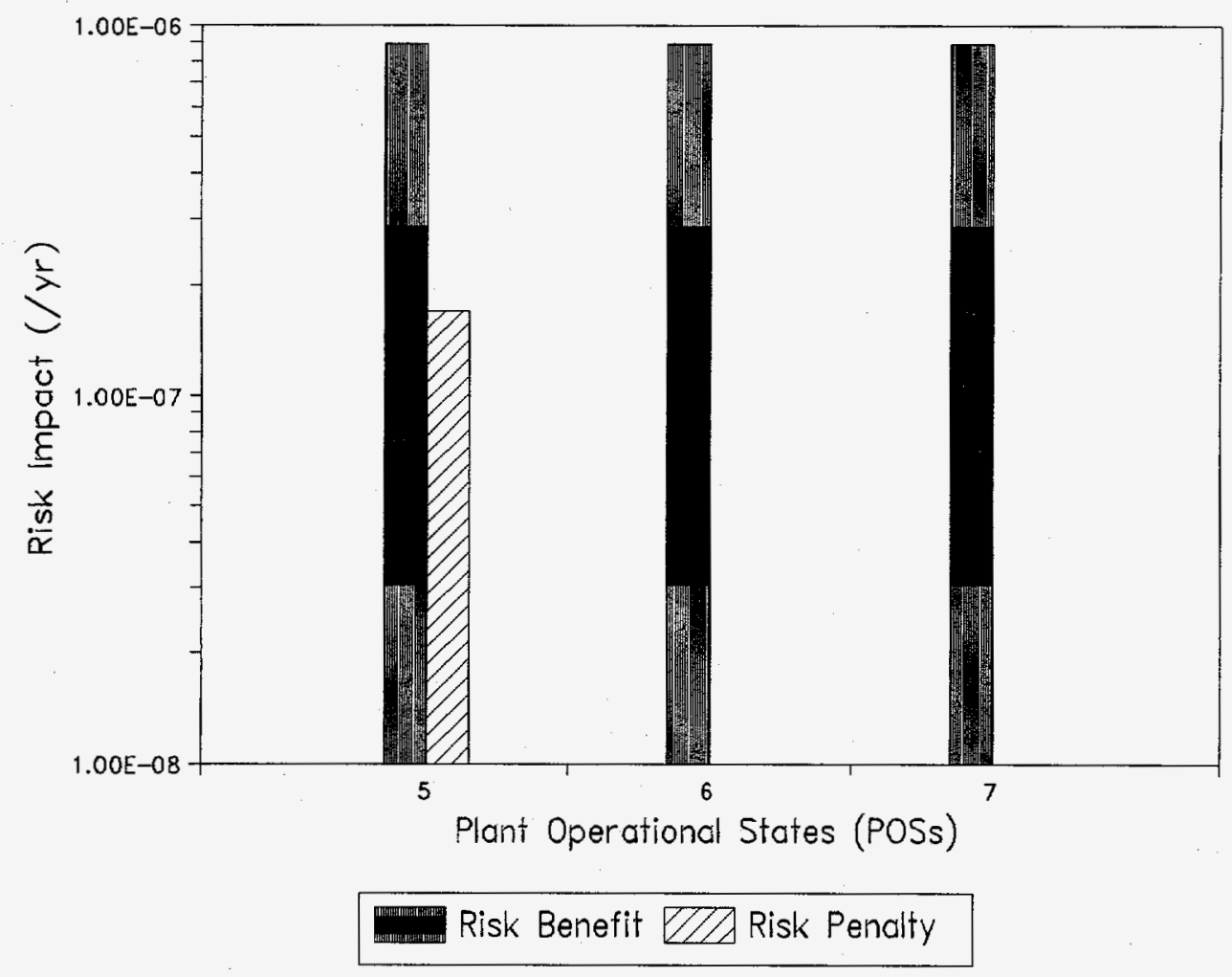

Figure 33. Risk Impact of the 18 Month Battery Emergency Load Discharge Test 


\subsubsection{Emergency Core Cooling System (ECCS) Surveillance}

During shutdown various tests are performed on the ECCS including the systems' flow tests, the systems' logic functional tests, and stroke and limit tests on various valves not normally tested at power. The system functional logic tests include a simulated LOCA signal with the purpose of testing the ECCS logic and verifying that the system automatically actuates.

\subsubsection{ECCS Flow Test}

The results of the risk impact of the ECCS flow tests are listed in Appendix D and shown in Figure 34 for HPCS. The results indicate that the Risk Benefit of the HPCS flow test is in the low range and is comparable in POS 5, 6, and 7. In all three POSs, the Risk Penalty due to Test Downtime is negligible. The results show similar trends for LPCS and LPCI A flow tests.

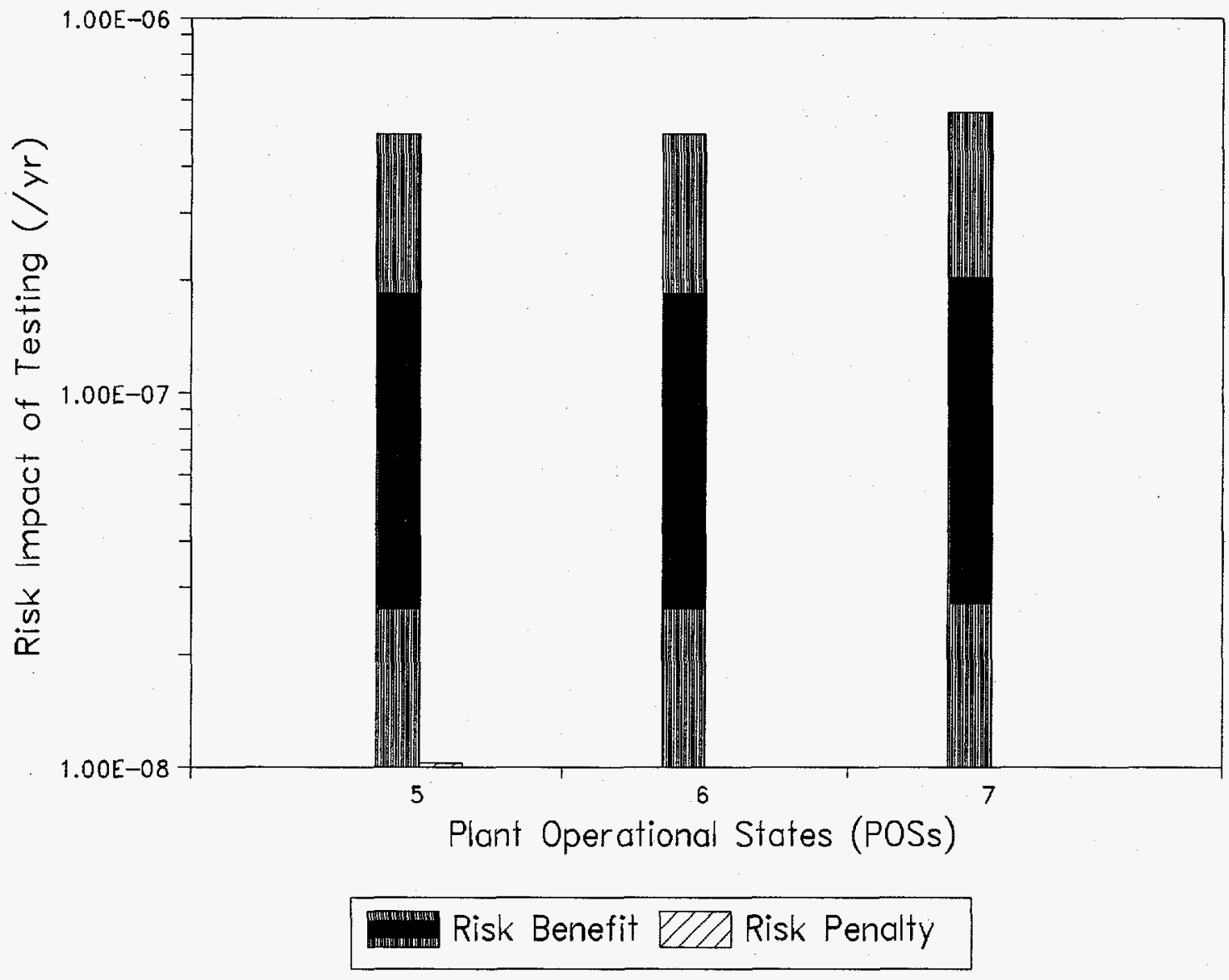

Figure 34. Risk Impact of the HPCS Quarterly Flow Test 


\subsubsection{ECCS Logic System Functional Test}

The results of the risk impact of the 18 month ECCS Logic System Functional tests are listed in Appendix D and shown in Figure 35 for LPCS. The results indicate that for LPCS, the Risk Benefit of performing the 18 month ECCS Logic System Functional tests at shutdown is medium while the Risk Penalty due to Test Downtime is low to negligible.
The results show similar trends for HPCS and LPCI A. Since the Risk Benefit of the ECCS 18 month functional test is in the medium range, the current STI of 18 months appears to be reasonable and might not be a candidate for extension unless an alternative testing schedule could be determined which could increase the STI without altering plant risk.

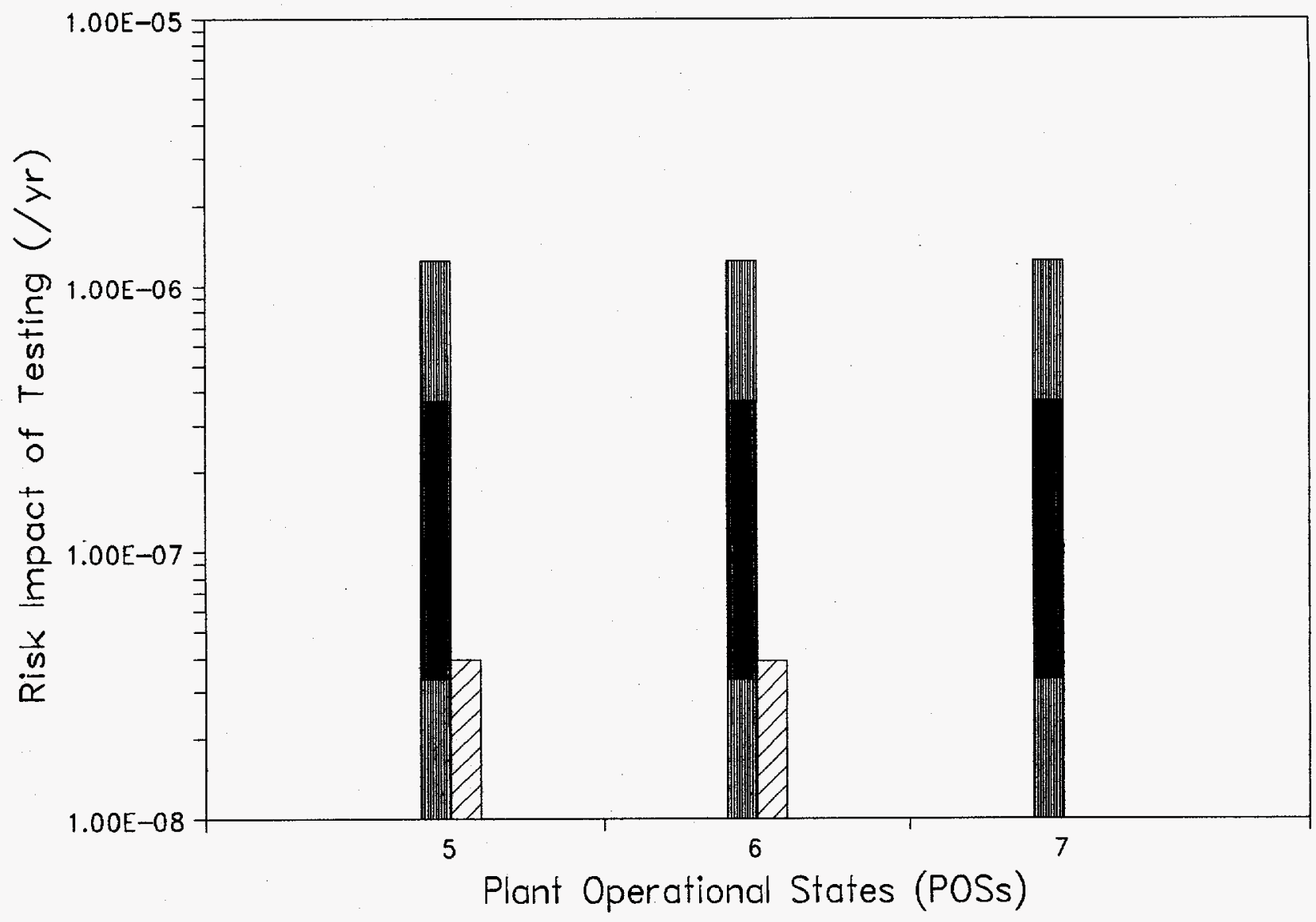

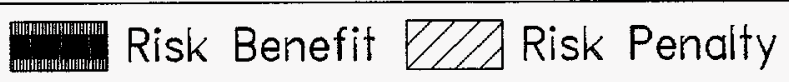

Figure 35. Risk Impact of the 18 Month Logic System Functional Test 


\subsubsection{ECCS 18 Month Valve Stroke Test}

The results of the risk impact of the ECCS 18 month valve stroke test is listed in Appendix D and shown in Figure 36 for LPCI testable check valve (TCV) 41A. The results indicate that for LPCI TCV 41A, the Risk Benefit of performing the 18 month valve stroke test at shutdown is low while the Risk Penalty due to Test Downtime is low to negligible. The results show similar trends for HPCS TCV 5 and LPCS TCV 6. Since the risk benefit of the 18 month stroke test is in the low range, the current STI of 18 months appears to be reasonable. However, this STI could possibly be considered for extension if an alternative testing schedule could be determined which would increase the STI without altering plant risk.

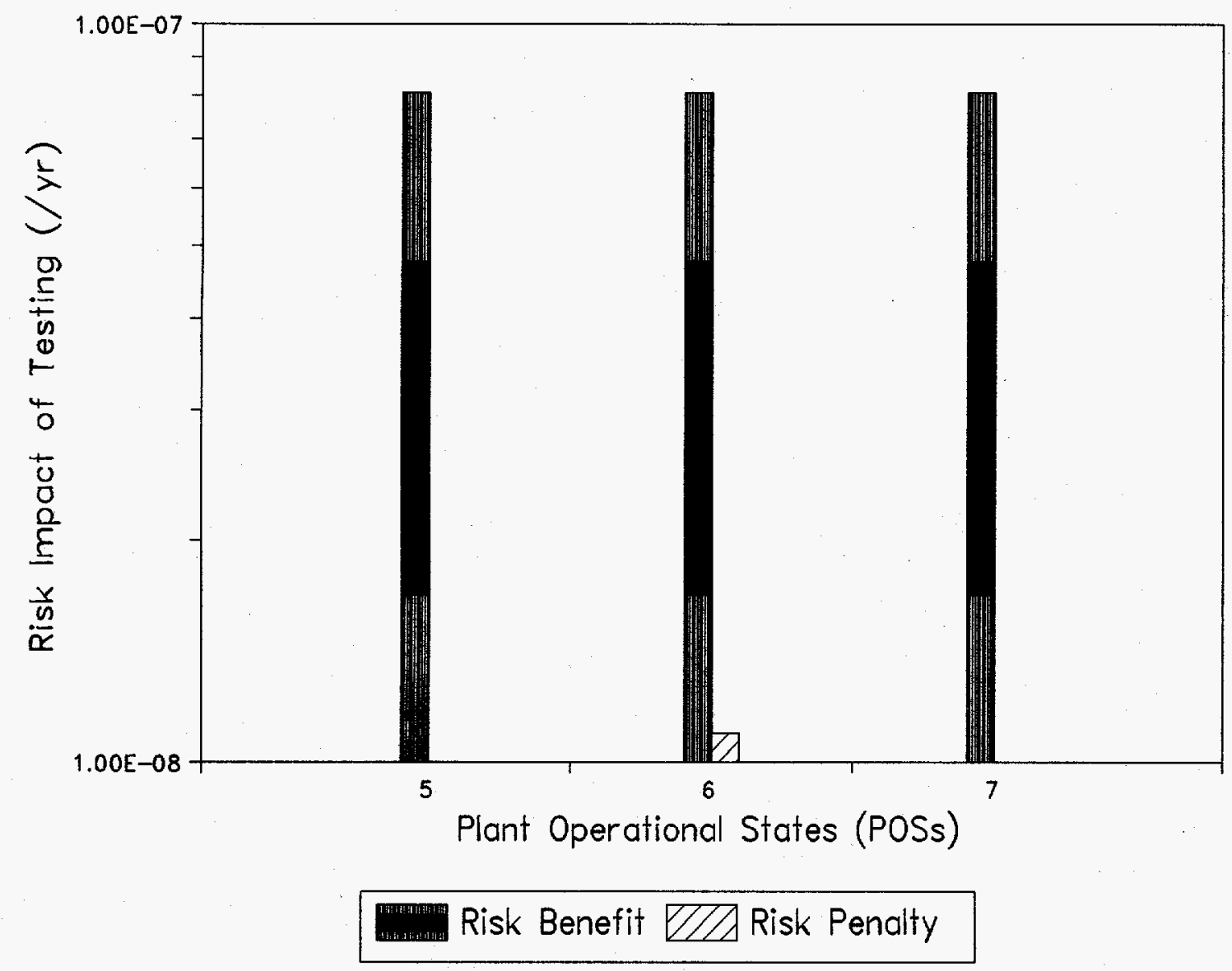

Figure 36. Risk Impact of the LPCI A 18 Month Stroke and Set Limits Test 


\subsection{Conclusions}

This section of the report contains general and specific conclusions on the risk impact of maintenance and test activities performed at power, shutdown, and refueling for GGNS. These conclusions are based on the results and insights derived in Sections 3 and 4 and may contain significant uncertainties resulting from limitations of the analysis (see Section 6.0). Any extrapolation of these results for use at plants other than GGNS should be done with caution so as not to undermine the safety of these plants.

The conclusions for the maintenance activities studied are as follows :

- Generally, the availability of safety systems is comparable or is of greater importance during low power and the early stages of shutdown than at power and refueling. Therefore, compared to power, there seems to be no risk advantage in scheduling preventative maintenance during low power and the early stages of shutdown.

- Generally, the AOTs specified in the TSs for preventative maintenance at power appear to reasonably control plant risk. However, the AOTs for maintenance on certain systems such as single Batteries, LPCS, and LPCI A could possibly be considered for extension. This could provide more time for proper maintenance without inducing significant increases in core damage probabilities.

- In certain cases, such as SSW and HPCS, CDF is sensitive to a single train out of service for maintenance. In these cases, limiting the frequency of planned maintenance might be considered, since repeated use of LCOs at power could cause significant increases in the Annual Increase in Core Damage Frequency.

- For some systems such as the EDGs and the batteries, a minimal level of availability is required at refueling with a high water level (POS 7). Therefore, preventative maintenance of long or uncertain duration should preferably be planned for refueling where the Increase in Conditional Core Damage Frequency is usually lower. However, for systems such as LPCI where the Increase in Conditional Core Damage Frequency at power appear to be less than or comparable to that at refueling, maintenance of long or uncertain duration could possibly be considered at power to reduce the burden of maintenance at refueling.
- Drainage of the suppression pool for maintenance or simultaneous maintenance on all SRVs results in high risk and should preferably be performed when the plant is in POS 6 or POS7 where water level is high and the vessel lid is removed.

- By defining an outage time at shutdown, such as the Outage Time at Refueling (OTR) that gives a core damage probability at refueling comparable to that at power, a relative, but non-binding, time frame can be used as a guide for scheduling preventative maintenance at shutdown as opposed to power. This could improve the correspondence between the TS requirements for power and shutdown.

- The Increase in Conditional Core Damage Frequency for single train maintenance on SSW during refueling is only slightly reduced compared to power. Thus, there is only a slight risk advantage to performing preventative maintenance of long duration at refueling as compared to power. By performing SSW maintenance at power, the maintenance burden at refueling could be reduced.

- Long term multiple train maintenance on SSW should preferably be scheduled at refueling where decay heat is low and ADHRS is available for decay heat removal. Maintenance should probably not be scheduled for the late phases of hot shutdown and the early phases of cold shutdown when ADHRS is not an alternative for decay heat removal. Any such activity would require the use of emergency procedures.

- For single train maintenance on LPCI/RHR, there appears to be no risk advantage to performing maintenance of long or short duration at refueling as compared to power . In addition, from a risk perspective, it appears preferable not to schedule maintenance for the late phases of hot shutdown and the early phases of cold shutdown when ADHRS is not an alternative for decay heat removal, unless the operability of an alternate train of DHR is confirmed.

- For multiple train maintenance on LPCI/RHR, there appears to be no risk advantage to performing maintenance of long or short duration at refueling as compared to power, and maintenance should preferably not be scheduled for the late phases of hot shutdown and the early phases of cold shutdown when ADHRS is not an alternative for decay heat removal. Any such activity would require the use of emergency procedures. 
- At power and shutdown, planned outage configurations and outage durations are key to controlling plant risk. Identifying maintenance activities and configurations which can lead to high risk and determining possible risk reducing alternatives can provide insight into possible areas which can aid in controlling risk at the plant.

The conclusions for the surveillance test activities studied are as follows :

- Performance of the monthly start test on the EDGs and the quarterly flow tests for the high pressure coolant systems (HPCS and RCIC) seem to be effective in controlling the potential risk at power. This is attributed to the fact that, at power, the dominant potential accidents are LOSP- initiated in which case use of the EDGs is crucial. In addition, if the reactor is pressurized, HPCS and RCIC are the only sources of coolant injection. The monthly start test on the EDGs and the quarterly flow tests for the high pressure coolant systems (HPCS and RCIC) tests do not appear to be candidates for STI extensions.

- At power, the Risk Benefit of quarterly flow testing the low pressure ECCS systems (LPCS and LPCI A) is small and appears to be less significant than that for the high pressure coolant systems. This is attributed to the fact that, at power where the dominant accident sequences are LOSP initiated, the low pressure systems can operate only if the reactor is depressurized. For these tests, extensions of the STIs could probably be considered with little risk increase to the plant. This could reduce the operational burden at the plant by allotting more time to accomplish more significant risk control activities.
- There is a low risk benefit associated with the quarterly SSW flow test at power. This is due to the fact that any failures in SSW can usually be detected during the frequent testing of other safety systems, such as the EDGs, which depend on SSW. Thus, the SSW flow test appears to be a possible candidate for STI extension.

- Since there is a low Risk Benefit associated with ECCS quarterly stroke tests and the weekly battery surveillance at power, extensions of their STIs could probably be considered with little risk increase to the plant. This could reduce the operational burden at the plant by allotting more time to accomplish more risk significant test activities.

- During shutdown and refueling, only a low to negligible Risk Penalty Due to Test Downtime is incurred with the performance of the ECCS flow test, the ECCS logic system functional test, the EDG $24 \mathrm{hr}$ run/load test, the EDG 18 month functional test simulating a loss of power (LOP), the ECCS 18 month valve stroke test and the 18 month battery emergency load test. Therefore, these tests can possibly be performed in POS 5,6 , or 7, preferably in POS 7.

- At shutdown and at refueling, the Risk Benefit of performing the EDG $24 \mathrm{hr}$ run test is significant (e.g. $1.57 \mathrm{E}-5 / \mathrm{yr}$ for EDGI). The Risk Benefit is medium for the ECCS logic system functional test but is low for the ECCS flow test, the EDG 18 month functional test (LOP), the ECCS 18 month valve stroke test, and the 18 month battery emergency load test. Even though the Risk Benefits of performing some of these tests at shutdown are low, the surveillance are necessary to assure system availability subsequently during power operations. 


\subsection{Limitations and Recommendations}

This section of the report contains a discussion of the limitations of the analysis and provides recommendations for future research on the risk impact of test and maintenance activities at power operations and shutdown.

\subsection{Limitations of the Analysis}

- The probabilistic models for POSs 4 through 7 were not scrutinized to the same degree as the NUREG/CR-4550 model used for POSs 0 through 3 . Therefore, minor shortcomings in the models for POS 4 s through 7 may have been overlooked.

- The analysis was performed using generic failure data; therefore, more accurate results could possibly be obtained by using plant specific data.

- The analysis did not consider external events such as internal fires and floods and may contain significant uncertainties.

- $\quad$ Since GGNS has some uncommon design safety features such as ADHRS, extrapolation of these results to other BWRs could require further analysis.

- The results, insights, and conclusions in this analysis are based solely on changes in CDFs and do not consider other factors such as economic implications and accident consequences (which are influenced by containment being open or closed).

\subsection{Recommendations for Model Improvement and Further Study}

- Improvements in the models for POS 1,2, and 3 can be made by refining the model for POS 0 (recall that POS 1, 2, and 3 are derived from POS 0). The model POS 0 can be improved by incorporating all additional initiators which were found to be important in the Grand Gulf IPE (Smith et al., 1992) or the LP\&SD study (Whitehead et al., 1994), for example, a loss of Instrument Air and a loss of Division I 4160 AC bus.
- In this study, POS 5 is limited to the first few hours of cold shutdown when the decay heat is still relatively high. However, during the middle and latter phases of cold shutdown, the decay heat decreases substantially. This could lead to a significant drop in the CDF in the latter phases of cold shutdown. Thus, a re-analysis of POS 5 by dividing the model for the POS into separate time windows covering a significant period could provide better insights into the risk impact of test and maintenance activities during cold shutdown. The development of time windows in POS 5 has been accomplished as well as applied in the LP\&SD study (Whitehead et al., 1994). Once the model for POS 5 is improved, the model for POS 4 could be improved as well, since POS 4 is developed from POS 5.

- Improvements in the models for POS 6 and POS 7 can be accomplished by modelling the POSs to the same level of detail as that done for the LP\&SD study (Whitehead et al., 1994), that is, incorporating reasonable values for operator and recovery actions.

- With the improved models (discussed above), this methodology could be applied to other BWRs by examining the sensitivities using the different plant configurations for other BWRs such as plants with two EDGS instead of three, plants with two trains of SSW instead of three, plants without ADHRS, and plants with 2 units sharing EDGS.

- Normally containment integrity, although required at power, is not specified in the TSs at shutdown and refueling except during fuel movement. However, a study of the effect of the status of the containment (open and closed) during certain test and maintenance activities at shutdown and refueling will facilitate more accurate comparisonsof scheduling maintenance during power operations versus during shutdown. 


\section{References}

Deem, R., et al., " A Screening Evaluation of PWR Shutdown Technical Specifications for the Surry Nuclear Power Plant," Draft, June 1992.

Drouin, M., et al., "Analysis of Core Damage Frequency: Grand Gulf Unit 1 Internal Events, "NUREG/CR-4550, September 1989.

Mankamo T., et al., "Optimization of Technical Specifications by Use of Probabilistic Methods," NKA RAS-450, May 1990.

Russell, D. K., et al., "Integrated Reliability and Risk Analysis System (IRRAS), " INEL Draft Report, December 1991.

Samanta, P. K., et al., "Evaluation of Risk Associated With AOT and STI Requirements at ANO-1 Nuclear Power Plant," NUREG/CR-5200, August 1988.

Samanta, P. K., et al., "Quantitative Evaluation of Surveillance Test Intervals Including Test Caused Risk," NUREG/CR-5577, February 1992.
Smith, G., et al., "Grand Gulf Nuclear Station Individual Plant Examination Summary Report," GGNS Report, December 1992.

Staple, B. D., et al., "The Risk Impact of Diesel Generator Unavailabilities for BWRs During Low Power and Shutdown," Letter Report, SNL, May 1993.

U.S. Nuclear Regulatory Commission (USNRC), " Technical Specifications -Grand Gulf Nuclear Station Unit No. 1, August 1990.

Whitehead, D. W., et al., "BWR Low Power and Shutdown Accident Sequence Frequencies Project for Grand Gulf Nuclear Station," SNL Draft Report, June 1991.

Whitehead, D. W., et al., "BWR Low Power and Shutdown Accident Sequence Frequencies Project : Phase 2 - Detailed Analysis of POS 5," SNL Draft Report, August 1992.

Whitehead, D. W., et al., "Evaluation of Potential Severe Accidents During Low Power and Shutdown Operations At Grand Gulf, Unit 1, Analysis of Core Damage Frequency from Internal Events for Plant Operational State 5 During a Refueling Outage," NUREG/CR-6143, July 1994. 


\section{APPENDIX A}

RAW DATA FOR EVALUATING RISK IMPACT OF SINGLE AND MULTIPLE MAINTENANCE ACTIVITIES 


\section{A.1 Analyses Performed for Each System or Subsystem Out for Maintenance}

This appendix contains a discussion of the analyses performed for each system or subsystem assumed to be out for maintenance and also presents tables of the raw data used in evaluating risk impacts of single and multiple maintenance activities.

\section{A.1.1 Plant Configurations for Single Train Maintenance}

The plant configurations of interest considering only single trains/systems being unavailable due to scheduled maintenance were as follows:

- Base Case: all systems/subsystems are available to mitigate the initiating events, i.e., no equipment is out for scheduled maintenance.

- EDG I: $\quad$ Emergency Diesel Generator I (Division I) in scheduled maintenance with all other systems/subsystems available to mitigate the initiating events.

- EDG II: $\quad$ Emergency Diesel Generator II (Division II) in scheduled maintenance with all other systems/subsystems available to mitigate the initiating events.

- EDG III: $\quad$ Emergency Diesel Generator III (Division II) in scheduled maintenance with all other systems/subsystems available to mitigate the initiating events.

- SSW A: $\quad$ Standby Service Water Train A in scheduled maintenance with all other systems/subsystems available to mitigate the initiating events.

- SSW C: $\quad$ Standby Service Water Train C in scheduled maintenance with all other systems/subsystems available to mitigate the initiating events.

- HPCS: High Pressure Core Spray in scheduled maintenance with all other systems/subsystems available to mitigate the initiating events.
- BAT I: Division I battery in scheduled maintenance with all other systems/subsystems available to mitigate the initiating events.

- BAT II: Division II battery in scheduled maintenance with all other systems/subsystems available to mitigate the initiating events.

- BAT III: Division III battery in scheduled maintenance with all other systems/subsystems available to mitigate the initiating events.

- RCIC: Reactor Core Isolation Cooling in scheduled maintenance with all other systems/subsystems available to mitigate the initiating events.

- CRD B: Control Rod Drive Train B in scheduled maintenance with all other systems/subsystems available to mitigate the initiating events.

- ADS: $\quad$ Automatic Depressurization System disabled for scheduled maintenance with all other systems/subsystems available to mitigate the initiating events.

- RHR A: Residual Heat Removal System Train $A$ in scheduled maintenance with all other systems/subsystems available to mitigate the initiating events.

- RHR C: Residual Heat Removal System Train $\mathrm{C}$ in scheduled maintenance with all other systems/subsystems available to mitigate the initiating events.

- LPCS: Low Pressure Core Spray in scheduled maintenance with all other systems/subsystems available to mitigate the initiating events.

- CDS: Condensate system in scheduled maintenance with all other systems/subsystems available to mitigate the initiating events.

- ISSP: $\quad$ Suppression Pool in scheduled maintenance (empty) with all other systems/subsystems available to mitigate the initiating events.

- SRVS: $\quad$ All Safety Relief Valves in scheduled maintenance with all other 
Appendix A

systems/subsystems available to mitigate the initiating events.

\section{A.1.2 Analyses Performed for Single Train Maintenance}

The following provides a brief discussion of the analyses performed for each of the systems/subsystems assumed to be in scheduled maintenance for each POS.

\section{A.1.2.1. POS 0 Single Train Maintenance Analysis}

Base Case: For the base case $\left(\mathrm{C}_{0}\right)$ all systems/subsystems are available, i.e., no systems/subsystems are in scheduled maintenance. To perform this analysis, all maintenance events were removed from the system fault trees and the dominate accident sequences were requantified to reflect the new plant configuration. Following quantification of the dominate accident sequences a recovery analysis was performed to complete the analysis.

EDG I: The Division I emergency diesel generator (EDG) is required to start and load following a loss of offsite power. Failure of the EDG I to start and load results in the loss of RHR Train A, SSW Train A, and LPCS. With EDG I out, the only emergency $A C$ power source following a Loss of Offsite Power (LOSP). would be Divisions II and III (HPCS diesel) given that EDG II and EDG III successfully start and load. Note that the Division III diesel can be cross-tied to either the Division I or Division II emergency AC bus if the HPCS pump fails to operate on demand or is otherwise unavailable. In this analysis, only those sequences in which offsite power is lost, since the diesel generators are needed only if offsite power is lost, were requantified in the base case. To perform this analysis, the fault tree logic for the Division I EDG was set to failed (availability set to 0 ), and all other equipment maintenance events were removed from the fault trees.

The Technical Specifications allow either Division I or II diesel to be removed from service in POS 0 for up to 72 hours as long as one of two of the diesels is operable.

EDG II: The Division II emergency diesel generator is required to start and load following a loss of offsite power. Failure of the EDG II to start and load results in the loss of RHR Trains B and $C$ and SSW Train B. With EDG II out, the only emergency $\mathrm{AC}$ power source following a LOSP would be Divisions I and III (HPCS diesel) given that EDG I and EDG III successfully start and load. Note that the Division III diesel can be cross-tied to either the Division I or Division II emergency AC bus if the HPCS pump fails to operate on demand or is otherwise unavailable. In this analysis, only those sequences which offsite power is lost, since the diesel generators are needed only if offsite power is lost, were requantified in the base case. To perform this analysis, the fault tree logic for the Division II EDG was set to failed (availability set to 0 ), and all other equipment maintenance events were removed from the fault trees.

The Technical Specifications allow either Division I or II diesel to be removed from service in POS 0 for up to 72 hours as long as one of two of the diesels is operable.

EDG III: The Division III emergency diesel generator is required to start and load following a LOSP. EDG III is dedicated to HPCS; therefore, failure of EDG III to start and load following a loss of offsite power would result in the loss of HPCS. With EDG III out, the only emergency $\mathrm{AC}$ power source following a LOSP would be Divisions I and II given that EDG I and EDG II successfully start and load. Note that the Division III diesel can be cross-tied to either the Division I or Division II emergency AC bus if the HPCS pump fails to operate on demand or is otherwise unavailable. In this analysis, only those sequences in which offsite power is lost, since the diesel generators are needed only if offsite power is lost, were requantified in the base case. To perform this analysis, the fault tree logic for the Division III EDG was set to failed (availability set to 0 ), and all other equipment maintenance events were removed from the fault trees.

Note that the Technical Specifications allow EDG III to be removed from service in POS 0 for up to 72 hours as long as EDG I and II are operable. If EDG III is not restored to operable status within 72 hours HPCS must be declared in operable. The Technical Specifications allow HPCS to be inoperable 
for up to 14 days as long as other modes of ECCS are operable. Therefore, EDG III could technically be out of service for up to seventeen days in POS 0 .

SSW A: SSW Train A is a support system which provides cooling water to RHR Train A, RCIC, LPCS, and EDG I. Only those sequences in the base case where the above systems are asked were requantified. To perform this analysis, the fault tree logic for RHR Train A, LPCS and EDG I was set to failed (availability set to 0 ), and all other equipment maintenance events were removed from the fault trees.

Note that SSW Train A provides cooling water to the RCIC room coolers (fan coil units). Based on the IPE, loss of RCIC room cooling would not fail RCIC within the 24 hour mission time considered in the analysis; therefore, this dependency was not modeled.

The Technical Specifications allow one train of SSW to be inoperable for up to 72 hours before requiring shutdown.

HPCS: HPCS is an Emergency Core Cooling System (ECCS) used to provide coolant to the core in the NUREG/CR-4550 study. Only those sequences in the base case where HPCS (note that EDG III is assumed not to be in scheduled maintenance) is asked (event tree top U1 )were requantified. To perform this analysis, the fault tree logic for HPCS was set to failed (availability set to 0), and all other equipment maintenance events were removed from the fault trees. With HPCS in scheduled maintenance, no other systems are made unavailable, HPCS and its supports are independent of all other systems.

Note that the Technical Specifications allow HPCS to be removed from service in POS 0 for up to 14 days as long as ECCS Divisions I and II and the RCIC system are operable.

SSW C: $\quad$ SSW Train C is a dedicated support system to HPCS which provides cooling water to EDG III and the HPCS room cooler (see the discussion of HPCS and EDG III above). In this analysis, it is assumed that HPCS is unavailable (tagged by the operator to be out of service) because of the lack of room cooling and that EDG III is unavailable (tagged out of service by the operator) because the diesel cannot operate without cooling water. To determine the core damage frequency of having SSW Train C in maintenance, the results of the EDG III analysis and the HPCS analysis were combined. This was possible since these analyses were independent of each other.

Note that the Technical Specifications allow SSW Train $\mathrm{C}$ to be removed from service in POS 0 for up to 14 days as long as ECCS Divisions I and II and the RCIC system are operable.

BAT-I: The Division I Battery (backed up by battery chargers) is required by the accident sequences considered to start the Division I diesel generator following a LOSP and also is required for RCIC operation following a LOSP. Because of this, the Division I battery only affects those sequences in which there is a LOSP. In this analysis, only those sequences in which a LOSP had occurred were requantified. To perform this analysis, the fault tree logic for the Division I battery, RCIC and Division I AC power was set to failed (availability set to 0 ), and all other equipment maintenance events were removed from the fault trees. No other sequences from the base case are affected by the Division I battery being out for scheduled maintenance.

Note that the Technical Specifications allow either Division I battery or Division II battery to be inoperable for up to 2 hours in POS 0 .

BAT-II: The Division II Battery (backed up by battery chargers) is required by the accident sequences considered to start the Division II diesel generator following a LOSP and also is required for RCIC operation following a LOSP. Because of this, the Division II battery only affects those sequences in which there is a LOSP. In this analysis, only those sequences in which a LOSP had occurred were requantified. To perform this analysis, the fault tree logic for the Division II battery, RCIC and Division II AC power was set to failed (availability set to 0 ), and all other equipment maintenance events were removed from the fault trees. No other sequences from the base case are affected by the Division II battery being out for scheduled maintenance. 
Appendix A

Note that the Technical Specifications allow either Division I battery or Division II battery to be inoperable for up to 2 hours in POS 1 .

BATT-III: The Division III Battery (backed up by battery chargers) is required by the accident sequences considered to start the Division III diesel generator following a LOSP and also is required to start the HPCS pump (i.e., close the pump breaker) following a LOSP. Because of this, the Division III battery only affects those sequences in which there is a LOSP. HPCS would be declared inoperable with the Division III battery out of service. However, in this analysis, HPCS is assumed available if offsite power is available; otherwise, this analysis would be the same as having SSW Train C out for maintenance, i.e., this analysis provides a measure of the significance of declaring HPCS unavailable given that Division III battery is out for maintenance while offsite power is available to provide DC power through the battery chargers. To perform this analysis, the fault tree logic for the Division III battery and Division III AC power was set to failed (availability set to 0 ) given a LOSP, and all other equipment maintenance events were removed from the fault trees. No other sequences from the base case are affected by the Division III battery being out for scheduled maintenance.

Note that the Technical Specifications allow Division III battery to be inoperable, but in doing so HPCS must be declared inoperable.

RCIC: $\quad$ RCIC is used for core cooling in the NUREG/CR-4550 study. Only those sequences in the base case where RCIC is asked (event tree top U2) were requantified. To perform this analysis, the fault tree logic for RCIC was set to failed (availability set to 0 ), and all other equipment maintenance events were removed from the fault trees. With RCIC in scheduled maintenance, no other systems are made unavailable.

Note that the Technical Specifications allow RCIC to be removed from service in POS 0 for up to 14 days as long as HPCS is operable.

CRD B: The CRD system was modeled in the NUREG/CR-4550 study as a backup source of high pressure coolant injection. In the NUREG/CR-4550 analysis, CRD Train A was assumed to be initially operating with Train B in standby. In the sequence of interest in this analysis, both trains of CRD are required for success. Since Train B is out of service, the event tree top for two pump CRD was set to failed, and the base case was requantified. With CRD Train B in scheduled maintenance, no other systems are made unavailable.

Note that there are no Technical Specifications for the CRD components required for coolant injection.

ADS: $\quad$ The ADS system is designed to automatically depressurize the reactor vessel when (1) a low water level signal is present in conjunction with a high drywell pressure signal and at least one low pressure pump is running after a 105 second timer or (2) a low water level signal is present and at least one low pressure pump is running after a ten minute time delay. In the NUREG/CR-4550 analysis, 3 of 8 valves working is considered success. In this analysis, the ADS valves are assumed out of service leaving only the nonADS SRVs, which must be manually operated, to depressurize the vessel. To perform this analysis, the fault tree logic for the ADS valves was set to failed (availability set to 0 ), and all other equipment maintenance events were removed from the fault trees.

Note that the Technical Specifications do not allow more than one ADS valve to be inoperable if the Steam Dome Pressure exceeds $135 \mathrm{psig}$. If more than one ADS valve is inoperable, the plant must be taken to Hot Shutdown within 12 hours and Cold Shutdown within the following 24 hours. One ADS valve may be inoperable for up to 14 days provided HPCS is operable and ECCS divisions I and II are operable. Note also that there are no Technical Specification requirements with the Steam Dome Pressure less than 135 psig.

RHR A: $\quad$ RHR Train A performs multiple functions which include LPCI, Shutdown Cooling (SDC), Containment Spray (CS), and Suppression Pool Cooling (SPC). In this 
analysis, RHR Train A is in scheduled maintenance with all other systems available. Only those sequences in the base case where RHR Train A functions (i.e., LPCS, SDC, CS, and/or SPC) are required were requantified. To perform this analysis, the fault tree logic for the various RHR Train A functions were set to failed (availability set to 0.0 ), and all other equipment maintenance events were removed from the fault trees. With RHR A in scheduled maintenance, no other systems are made unavailable.

Note that the Technical Specifications allow LPCI Train A to be unavailable for up to seven days in POS 0 as long as all other divisions of ECCS are available.

RHR C: $\quad$ RHR Train C performs but one function, unlike RHR Train A, which is Low Pressure Coolant Injection (LPCI). In this analysis, LPCI $C$ is in scheduled maintenance with all other systems available. Only those sequences in the base case where LPCI C is required were requantified. To perform this analysis, the fault tree logic for LPCI C was set to failed (availability set to 0.0 ), and all other equipment maintenance events were removed from the fault trees. With RHR C in scheduled maintenance, no other systems are made unavailable.

Note that the Technical Specifications allow LPCI $C$ to be unavailable for up to seven days in POS 0 as long as all other divisions of ECCS are available.

LPCS: $\quad$ LPCS is an ECCS system used for core cooling in the NUREG/CR-4550 and IPE studies. Only those sequences in the base case where LPCS was asked were requantified. To perform this analysis, the fault tree logic for LPCS was set to failed (availability set to 0 ), and all other equipment maintenance events were removed from the fault trees. With LPCS in scheduled maintenance, no other systems are made unavailable.

Note that the Technical Specifications allow LPCS to be unavailable for up to seven days in POS 0 as long as all other divisions of ECCS are available.
The results of the single train maintenance analysis for POS 0 are given in Table A.1.

\section{A.1.2.2 POS 1 Single Train Maintenance Analysis}

In POS 1, the plant is in a low power condition (i.e., thermal power $\leq 15 \%$ at rated pressure $\approx 1000 \mathrm{psig}$ ) and is assumed to be shutting down for refueling. Since the plant is still at power in POS 1 and not shutdown, it was assumed that the conditions in POS 1 could be modeled using the POS 0 full power model. The only difference between the POS 0 model and the POS 1 model is the initiating event frequency for a LOSP (T1) (0.1/yr in POS 0 and 0.07 in POS 1) which was incorporated into the POS 1 model. Therefore, the POS 1 results are the POS 0 results with initiating frequency for $\mathrm{T} 1 \mathrm{changed}$ from $0.1 / \mathrm{yr}$ to $0.07 / \mathrm{yr}$.

The results of the single train maintenance analysis for POS 1 are given in Table A.1.

\section{A.1.2.3 POS 2/3 Single Train Maintenance Analysis}

In POS 2 and POS 3, the plant is in hot shutdown with all rods inserted into the core, it is assumed that the plant is shutting down for refueling. The RCIC system is still available for injection in both POS 2 and POS 3.

It was assumed that both POS 2 and POS 3 could be modeled as one utilizing the POS 0 model with minor revisions. These revisions included eliminating the ATWS accident sequence since the plant is shutdown, eliminating T1 sequences 17 and 21 because it was assumed the SRVs would not open for reactor coolant system (RCS) overpressurization protection in POS 2 or POS 3 making stuck open relief valve failures invalid (i.e, no pressure surge in the vessel due to reactor scram), and revising the initiating event frequency for $\mathrm{T} 1$ from $0.1 / \mathrm{yr}$ to $0.13 / \mathrm{yr}$.

The results of the single train maintenance analysis for POS $2 / 3$ are given in Table A.1.

\section{A.1.2.4 POS 4 Single Train Maintenance Analysis}

POS 4 is a transition phase between POS 3 and POS 5 where RCIC is no longer assumed to be available (RCIC declared inoperable at $135 \mathrm{psig}$ ), and the operator is switching to SDC for core cooling. Because core cooling is being achieved with SDC, POS 4 is similar to POS 5; therefore, the PRA model for POS 4 is a revised model of POS 5. Two major revisions to the POS 5 model were made to develop a POS 4 model; 1) the elimination of all sequences where ADHRS is initially operating providing core cooling (ADHRS cannot successfully cool the core in POS 4) and 2) the elimination of all sequences where the 
containment is initially open, i.e., it is assumed that the containment is not yet opened for maintenance in POS 4, hot shutdown (see Section A.1.2.5 below for a complete discussion of the systems looked at and descriptions of the models used in the analysis of single train maintenance in POS 4).

The results of the single train maintenance analysis for POS 4 are given in Table A.2.

\section{A.1.2.5 POS 5 Single Train Maintenance Analysis}

Base Case: For the base case $\left(\mathrm{C}_{0}\right)$ all systems/subsystems are available, i.e., no systems/subsystems are in scheduled maintenance. To perform this analysis, all maintenance events were removed from the system fault trees, and the dominate accident sequences were requantified to reflect the new plant configuration. Following quantification of the dominate LP\&SD study accident sequences, a recovery analysis was performed to complete the analysis.

SSW C: $\quad$ SSW Train C is a dedicated support system to HPCS which provides cooling water to EDG III and the HPCS room cooler (see the discussion of HPCS and EDG III below). In this analysis, it is assumed that HPCS is unavailable (tagged by the operator to be out of service) because of the lack of room cooling and that EDG III is unavailable (tagged out of service by the operator) because the diesel cannot operate without cooling water. To perform this analysis, the fault tree logic for HPCS and EDG III was set to failed (availability set to 0.0 ), and all other equipment maintenance events were removed from the fault trees.

CDS: $\quad$ CDS is used for level control and for low pressure steaming of the core in the LP\&SD study. CDS in the LP\&SD study was assumed to be unavailable $40 \%$ of the time. In this analysis, CDS is out for scheduled maintenance with all other systems available. Only those sequences in the base case where CDS is asked were requantified. To perform this analysis, the fault tree logic for CDS was set to failed (availability set to 0 ), and all other equipment maintenance events were removed from the fault trees.
With CDS in scheduled maintenance, no other systems are made unavailable.

CRD B: $\quad$ CRD is used for level control and for low pressure and high pressure steaming operations in the LP\&SD study. In this analysis, CRD Train $B$ is in scheduled maintenance, and all other systems are available. CRD Train $A$ is operating in makeup mode (i.e., level control). Only those sequences in the base case where CRD is asked were requantified. To perform this analysis, the fault tree logic for CRD Train B was set to failed (availability set to 0), and all other equipment maintenance events were removed from the fault trees. With CRD Train B in scheduled maintenance, no other systems are made unavailable.

EDG I: $\quad$ Emergency diesel generator Division $I$ is required to start and load following a LOSP. Failure of the EDG I to start and load results in the loss of RHR Train A, SSW Train A, and LPCS. With EDG I out, the only emergency $\mathrm{AC}$ power source following a LOSP would be Divisions II and III (HPCS diesel) given that EDG II and EDG III successfully start and load. Note that the Division III diesel can be cross-tied to either the Division I or Division II emergency AC bus if the HPCS pump fails to operate on demand or is otherwise unavailable. In this analysis, only those sequences in which offsite power is lost, since the diesel generators are needed only if offsite power is lost, were requantified in the base case. To perform this analysis, the fault tree logic for Division I was set to failed (availability set to 0 ), and all other equipment maintenance events were removed from the fault trees.

Note that the Technical Specifications allow either Division I or II diesel to be removed from service in POS 5, but not both.

EDG II: $\quad$ Emergency diesel generator Division II is required to start and load following a LOSP. Failure of the EDG II to start and load results in the loss of RHR Trains B and C and SSW Train B. With EDG II out, the only emergency $\mathrm{AC}$ power source following a LOSP would be Divisions I and III (HPCS diesel) given that EDG I and EDG III successfully start and load. Note that the 
Division III diesel can be cross-tied to either the Division I or Division II emergency AC bus if the HPCS pump fails to operate on demand or is otherwise unavailable. In this analysis, only those sequences in which offsite power is lost, since the diesel generators are needed only if offsite power is lost, were requantified in the base case. To perform this analysis, the fault tree logic for Division II was set to failed (availability set to 0), and all other equipment maintenance events were removed from the fault trees.

Note that the Technical Specifications allow either Division I or II diesel to be removed from service in POS 5, but not both.

HPCS: HPCS is an Emergency Core Cooling System (ECCS) used for level control, water solid closed loop core cooling, and high pressure steaming operations in the LP\&SD study. HPCS in the LP\&SD study was assumed to be unavailable $20 \%$ of the time. In this analysis, HPCS is in scheduled maintenance and all other systems are available. Only those sequences in the base case where HPCS is asked were requantified. To perform this analysis, the fault tree logic for HPCS was set to failed (availability set to 0 ), and all other equipment maintenance events were removed from the fault trees. With HPCS in scheduled maintenance, no other systems are made unavailable since HPCS and its supports are independent of all other systems.

BAT-I: The Division I Battery (backed up by battery chargers) is required to start the Division I diesel generator and the Division I ECCS system (LPCS and LPCI A) pumps following a LOSP. Because of this, the Division I battery only affects those sequences in which there is a LOSP. In this analysis, only those sequences in which a LOSP had occurred were requantified. To perform this analysis, the fault tree logic for the Division I battery and EDG I were set to failed (availability set to 0 ), and all other equipment maintenance events were removed from the fault trees. No other sequences from the base case are affected by the Division I battery being out for scheduled maintenance, i.e., a review of the cut sets showed no Division I battery failures contributing to core damage.
The net effect of having the Division I Battery in scheduled maintenance is the same as having EDG III in scheduled maintenance, given a LOSP.

BAT-II: $\quad$ The Division II Battery (backed up by battery chargers) is required to start the Division II diesel generator and the Division II ECCS system (LPCI Trains A and B) pumps following a LOSP. Because of this, the Division II battery only affects those sequences in which there is a LOSP. In this analysis, only those sequences in which a LOSP had occurred were requantified. To perform this analysis, the fault tree logic for the Division II battery and EDG II were set to failed (availability set to 0 ), and all other equipment maintenance events were removed from the fault trees. No other sequences from the base case are affected by the Division II battery being out for scheduled maintenance, i.e., a review of the cut sets showed no Division II battery failures contributing to core damage.

The net effect of having the Division II Battery in scheduled maintenance is the same as having EDG II in scheduled maintenance, given a LOSP.

BAT-III: The Division III Battery (backed up by battery chargers) is required to start the Division III diesel generator and the HPCS pump following a LOSP. Because of this, the Division III battery only affects those sequences in which there is a LOSP followed by failure of the Division I and II. In this analysis, only those sequences in which a LOSP had occurred followed by failure of Divisions I and II diesels were requantified. To perform this analysis, the fault tree logic for the Division III battery (HPCS) was set to failed (availability set to 0 ), and all other equipment maintenance events were removed from the fault trees. No other sequences from the base case are affected by the Division III battery being out for scheduled maintenance.

The net effect of having the Division III Battery in scheduled maintenance is the same as having EDG III in scheduled maintenance, given a LOSP. 
Appendix A

ISSP: The Suppression Pool provides the suction source for all ECCS and also acts as a heat sink for the removal of decay heat from the core. It is not expected that the suppression pool would ever be completely drained for maintenance; however, this analysis 'was done to determine the vulnerability of the plant should it be drained. Those sequences in the base case where the Suppression Pool is empty and/or where ECCS systems are asked were requantified with the ECCS systems (i.e., HPCS, LPCS, and LPCI) set to failed (availability set to 0 ). All other equipment maintenance events were removed from the fault trees.

RHR A: $\quad$ RHR Train A performs multiple functions in the LP\&SD study which include LPCI, SDC, Containment Spray (CS), and Suppression Pool Cooling (SPC). In this analysis, RHR Train A is in scheduled maintenance, and all other systems are available. Only those sequences in the base case where RHR Train A functions are asked were requantified. To perform this analysis, the fault tree logic for the various RHR Train A functions were set to failed (availability set to 0 ), and all other equipment maintenance events were removed from the fault trees. With RHR Train A in scheduled maintenance, no other systems are made unavailable.

RHR C: $\quad$ RHR Train C performs but one function, unlike RHR Train A, which is LPCI for level control and for water solid closed loop core cooling. In this analysis, RHR Train C is in scheduled maintenance and all other systems are available. Only those sequences in the base case where RHR Train C is asked were requantified. To perform this analysis, the fault tree logic for RHR Train C was set to failed (availability set to 0 ), and all other equipment maintenance events were removed from the fault trees. With RHR Train C in scheduled maintenance, no other systems are made unavailable.

SSW A: $\quad$ SSW Train A is a support system which provides cooling water to RHR Train A, LPCS, and EDG I (see discussion of RHR Train A above). LPCS is used in the LP\&SD study for level control and for water solid closed loop core cooling, while EDG I is required to operate following a LOSP to power Division A components. In this analysis, RHR Train A, LPCS, and EDG I are in scheduled maintenance, and all other systems are available. Only those sequences in the base case where RHR Train A functions, LPCS, and/or EDG I are asked were requantified. To perform this analysis, the fault tree logic for RHR Train A, LPCS, and EDG I was set to failed (availability set to 0), and all other equipment maintenance events were removed from the fault trees.

SRVS: $\quad$ Normally during LP\&SD conditions at least 2 SRVs are required to be operational. The SRVs perform an important role in the LP\&SD study should SDC be lost. The SRVs are required for water solid closed loop core cooling, containment flooding, and steaming the core at low and high pressure. Without the SRVs, none of these core cooling functions could be performed. For this analysis, the affect of having no SRVs available could not be determined using the LP\&SD study dominate accident sequences since no surviving sequences had SRV failures. Therefore, in order to perform this analysis the following assumptions had to be made:

- Given SDC lost, core damage (CD) would occur since no SRVs are available to perform core cooling operations such as water solid closed loop core cooling, steaming the core at low or high pressure, and flooding the containment.

Given the above assumption, the sequence logic for the dominate LP\&SD study CD sequences were modified to consider only those sequences in which SDC was lost. Upon loss of SDC, CD was assumed.

It is not expected that a plant would remove all SRVs from service without a contingency plan should SDC be lost while all SRVs are in scheduled maintenance. There are other means to control vessel pressure and provide makeup for steaming besides the SRVs such as opening the RCIC steam line to the SP, the main steam line drain valves, and the condenser through the main steam lines. Credit was given in this analysis for such a contingency plan (OP-DEP) and was given a screening value of 0.1 . This credit was only 
given in those sequences in which the operator had recognized the loss of SDC, which is explicitly modeled in the sequences.

LPCS: $\quad$ LPCS is an ECCS system used for level control and water solid closed loop core cooling in the LP\&SD study. In this analysis, LPCS is in scheduled maintenance, and all other systems are available. Only those sequences in the base case where LPCS is asked were requantified. To perform this analysis, the fault tree logic for LPCS was set to failed (availability set to 0 ), and all other equipment maintenance events were removed from the fault trees. With LPCS in scheduled maintenance, no other systems are made unavailable.

The results of the single train maintenance analysis for POS 5 are given in Table A.2.

\section{A.1.2.6 POS 6 Single Train Maintenance Analysis}

Base Case: For the base case $\left(C_{0}\right)$ all systems/subsystems are available, i.e., no systems/subsystems are in scheduled maintenance. To perform this analysis, all maintenance events were removed from the system fault trees, and the dominate accident sequences were requantified to reflect the new plant configuration. Following quantification of the dominate accident sequences, a recovery analysis was performed to complete the analysis.

SSW C: $\quad$ SSW Train C is a dedicated support system to HPCS which provides cooling water to EDG III and the HPCS room cooler (see the discussion of HPCS and EDG III below). In this analysis, it is assumed that HPCS is unavailable (tagged by the operator to be out of service) because of the lack of room cooling and that EDG III is unavailable (tagged out of service by the operator) because the diesel cannot operate without cooling water. To perform this analysis, the fault tree logic for HPCS and EDG III was set to failed (availability set to 0.0 ), and all other equipment maintenance events were removed from the fault trees.

CDS: $\quad$ CDS is used for level control and core cooling operations in the POS 6 analysis. In this analysis, CDS is out for scheduled maintenance with all other systems available.
Only those sequences in the base case where CDS is asked were requantified. To perform this analysis, the fault tree logic for CDS was set to failed (availability set to 0 ), and all other equipment maintenance events were removed from the fault trees. With CDS in scheduled maintenance, no other systems are made unavailable.

CRD B: $\quad$ CRD is used for level control and for core cooling operations in the POS 6 analysis. In this analysis, CRD Train $B$ is in scheduled maintenance and all other systems are available, CRD Train $A$ is operating in makeup mode (i.e., level control). Only those sequences in the base case where CRD is asked were requantified. To perform this analysis, the fault tree logic for CRD Train B was set to failed (availability set to 0 ), and all other equipment maintenance events were removed from the fault trees. With CRD Train B in scheduled maintenance, no other systems are made unavailable.

EDG I: $\quad$ Emergency diesel generator Division $I$ is required to start and load following a LOSP. Failure of the EDG I to start and load results in the loss of RHR Train A, SSW Train A, and LPCS. With EDG I out, the only emergency $\mathrm{AC}$ power source following a LOSP would be Divisions II and III (HPCS diesel) given that EDG II and EDG III successfully start and load. Note that the Division III diesel can be cross-tied to either the Division I or Division II emergency AC bus if the HPCS pump fails to operate on demand or is otherwise unavailable. In this analysis, only those sequences in which offsite power is lost, since the diesel generators are needed only if offsite power is lost, were requantified in the base case. To perform this analysis, the fault tree logic for Division I EDG was set to failed (availability set to 0 ), and all other equipment maintenance events were removed from the fault trees.

Note that the Technical Specifications allow either Division I or II diesel to be removed from service in POS 6, but not both.

EDG II: Emergency diesel generator Division II is required to start and load following a LOSP. Failure of the EDG II to start and load 
results in the loss of RHR Trains B and C and SSW Train B. With EDG II out, the only emergency $A C$ power source following a LOSP would be Divisions I and III (HPCS diesel) given that EDG I and EDG III successfully start and load. Note that the Division III diesel can be cross-tied to either the Division I or Division II emergency AC bus if the HPCS pump fails to operate on demand or is otherwise unavailable. In this analysis, only those sequences in which offsite power is lost, since the diesel generators are needed only if offsite power is lost, were requantified in the base case. To perform this analysis, the fault tree logic for Division II EDG was set to failed (availability set to 0 ), and all other equipment maintenance events were removed from the fault trees.

Note that the Technical Specifications allow either Division I or II diesel to be removed from service in POS 6, but not both.

HPCS: HPCS is an Emergency Core Cooling System (ECCS) used for level control in the POS 6 analysis. In this analysis, HPCS is in scheduled maintenance and all other systems are available. Only those sequences in the base case where HPCS is asked were requantified. To perform this analysis, the fault tree logic for HPCS was set to failed (availability set to 0 ), and all other equipment maintenance events were removed from the fault trees. With HPCS in scheduled maintenance, no other systems are made unavailable since HPCS and its supports are independent of all other systems.

BAT-I: The Division I Battery (backed up by battery chargers) is required to start the Division I diesel generator and the Division I ECCS systems (LPCS and LPCI A) pump following a LOSP. Because of this, the Division I battery only affects those sequences in which there is a LOSP. In this analysis, only those sequences in which a LOSP had occurred were requantified. To perform this analysis, the fault tree logic for the Division I battery and EDG I were set to failed (availability set to 0 ), and all other equipment maintenance events were removed from the fault trees. No other sequences from the base case are affected by the Division I battery being out for scheduled maintenance, i.e., a review of the cut sets showed no Division I Battery failures contributing to core damage.

The net effect of having the Division I Battery in scheduled maintenance is the same as having EDG III in scheduled maintenance, given a LOSP.

BAT-II: The Division II Battery (backed up by battery chargers) is required to start the Division II diesel generator and the Division II ECCS systems (LPCI Trains A and B) pump following a LOSP. Because of this, the Division II battery only affects those sequences in which there is a LOSP. In this analysis, only those sequences in which a LOSP had occurred were requantified. To perform this analysis, the fault tree logic for the Division II battery and EDG II were set to failed (availability set to 0 ), and all other equipment maintenance events were removed from the fault trees. No other sequences from the base case are affected by the Division II battery being out for scheduled maintenance, i.e., a review of the cut sets showed no Division II battery failures contributing to core damage.

The net effect of having the Division II Battery in scheduled maintenance is the same as having EDG II in scheduled maintenance, given a LOSP.

BAT-III: The Division III Battery (backed up by battery chargers) is required to start the Division III diesel generator and the HPCS pump following a LOSP. Because of this, the Division III battery only affects those sequences in which there is a LOSP followed by failure of the Division I and II. In this analysis, only those sequences in which a LOSP had occurred followed by failure of Divisions I and II diesels were requantified. To perform this analysis, the fault tree logic for the Division III battery (HPCS) was set to failed (availability set to 0 ), and all other equipment maintenance events were removed from the fault trees. No other sequences from the base case are affected by the Division III battery being out for scheduled maintenance.

The net effect of having the Division III Battery in scheduled maintenance is the same as having EDG III in scheduled maintenance, given a LOSP.

ISSP: The Suppression Pool provides the suction source for all ECCS and also acts as a heat sink for the 
removal of decay heat from the core. It is not expected that the suppression pool would ever be completely drained for maintenance; however, this analysis was done to determine the vulnerability of the plant should it be drained. Those sequences in the base case where the SP is empty and/or where ECCS systems are asked were requantified with the ECCS systems (i.e., HPCS, LPCS, and LPCI) set to failed (availability set to 0 ). All other equipment maintenance events were removed from the fault trees.

RHR A: $\quad$ RHR Train A performs multiple functions which include LPCI, SDC, CS, and SPC. In this analysis, RHR Train A is in scheduled maintenance and all other systems are available. Only those sequences in the base case where RHR Train A functions are asked were requantified. To perform this analysis, the fault tree logic for the various RHR Train A functions was set to failed (availability set to 0 ), and all other equipment maintenance events were removed from the fault trees. With RHR Train A in scheduled maintenance, no other systems are made unavailable.

RHR C: $\quad$ RHR Train C performs but one function, unlike RHR Train A, which is LPCI for level control. In this analysis, RHR Train C is in scheduled maintenance, and all other systems are available. Only those sequences in the base case where RHR Train C is asked were requantified. To perform this analysis, the fault tree logic for RHR Train C was set to failed (availability set to 0 ), and all other equipment maintenance events were removed from the fault trees. With RHR Train C in scheduled maintenance, no other systems are made unavailable.

SSW A: $\quad$ SSW Train A is a support system which provides cooling water to RHR Train A, LPCS, and EDG I (see discussion of RHR Train A above). LPCS is used for level control in the POS 6 analysis while EDG $I$ is required to operate following a LOSP to power Division A components. In this analysis RHR Train A, LPCS, and EDG I are in scheduled maintenance and all other systems are available. Only those sequences in the base case where RHR Train A functions, LPCS, and/or EDG I are asked were requantified. To perform this analysis, the fault tree logic for RHR Train A, LPCS, and EDG I was set to failed (availability set to 0 ), and all other equipment maintenance events were removed from the fault trees.

LPCS: $\quad$ LPCS is an ECCS system used for level control in the POS 6 analysis. In this analysis, LPCS is in scheduled maintenance and all other systems are available. Only those sequences in the base case where LPCS is asked were requantified. To perform this analysis, the fault tree logic for LPCS was set to failed (availability set to 0 ), and all other equipment maintenance events were removed from the fault trees. With LPCS in scheduled maintenance, no other systems are made unavailable.

The results of the single train maintenance analysis for POS 6 are given in Table A.2.

\section{A.1.2.7 POS 7 Single Train Maintenance Analysis}

Base Case: For the base case $\left(\mathrm{C}_{0}\right)$ all

systems/subsystems are available, i.e., no systems/subsystems are in scheduled maintenance. To perform this analysis, all maintenance events were removed from the system fault trees, and the dominate accident sequences were requantified to reflect the new plant configuration. Following quantification of the dominate accident sequences, a recovery analysis was performed to complete the analysis.

SSW C: $\quad$ SSW Train C is a dedicated support system to HPCS which provides cooling water to EDG III and the HPCS room cooler (see the discussion of HPCS and EDG III below). In this analysis, it is assumed that HPCS is unavailable (tagged by the operator to be out of service) because of the lack of room cooling and that EDG III is unavailable (tagged out of service by the operator) because the diesel cannot operate without cooling water. To perform this analysis, the fault tree logic for HPCS and EDG III was set to failed (availability set to 0.0 ), and all other equipment maintenance events were removed from the fault trees.

CDS: $\quad$ CDS is used for level control and core cooling operations in the POS 7 analysis. In this analysis, CDS is out for scheduled 
Appendix A

maintenance with all other systems available. Only those sequences in the base case where CDS is asked were requantified. To perform this analysis, the fault tree logic for CDS was set to failed (availability set to 0 ), and all other equipment maintenance events were removed from the fault trees. With CDS in scheduled maintenance, no other systems are made unavailable.

CRD B: $\quad$ CRD is used for level control and for core cooling operations in the POS 7 analysis. In this analysis, CRD Train B is in scheduled maintenance and all other systems are available, CRD Train $A$ is operating in makeup mode (i.e., level control). Only those sequences in the base case where CRD is asked were requantified. To perform this analysis, the fault tree logic for CRD Train B was set to failed (availability set to 0 ), and all other equipment maintenance events were removed from the fault trees. With CRD Train B in scheduled maintenance, no other systems are made unavailable.

EDG I: $\quad$ Emergency diesel generator Division I is required to start and load following a LOSP. Failure of the EDG I to start and load results in the loss of RHR Train A, SSW Train A, and LPCS. With EDG I out, the only emergency $A C$ power source following a LOSP would be Divisions II and III (HPCS diesel) given that EDG II and EDG III successfully start and load. Note that the Division III diesel can be cross-tied to either the Division I or Division II emergency AC bus if the HPCS pump fails to operate on demand or is otherwise unavailable. In this analysis, only those sequences in which offsite power is lost, since the diesel generators are needed only if offsite power is lost, were requantified in the base case. To perform this analysis, the fault tree logic for Division I EDG was set to failed (availability set to 0), and all other equipment maintenance events were removed from the fault trees.

Note that the Technical Specifications allow either Division I or II diesel to be removed from service in POS 7, but not both.
EDG II: $\quad$ Emergency diesel generator Division II is required to start and load following a LOSP. Failure of the EDG II to start and load results in the loss of RHR Trains B and C and SSW Train B. With EDG II out, the only emergency $A C$ power source following a LOSP would be Divisions I and III (HPCS diesel) given that EDG I and EDG III successfully start and load. Note that the Division III diesel can be cross-tied to either the Division I or Division II emergency AC bus if the HPCS pump fails to operate on demand or is otherwise unavailable. In this analysis, only those sequences in which offsite power is lost, since the diesel generators are needed only if offsite power is lost, were requantified in the base case. To perform this analysis, the fault tree logic for Division II EDG was set to failed (availability set to 0 ), and all other equipment maintenance events were removed from the fault trees.

Note that the Technical Specifications allow either Division I or II diesel to be removed from service in POS 7, but not both.

HPCS: $\quad$ HPCS is an Emergency Core Cooling System (ECCS) used for level control and core cooling in the POS 7 analysis. In this analysis, HPCS is in scheduled maintenance, and all other systems are available. Only those sequences in the base case where HPCS is asked were requantified. To perform this analysis, the fault tree logic for HPCS was set to failed (availability set to 0), and all other equipment maintenance events were removed from the fault trees. With HPCS in scheduled maintenance, no other systems are made unavailable since HPCS and its supports are independent of all other systems.

BAT-I: $\quad$ The Division I Battery (backed up by battery chargers) is required to start the Division I diesel generator and the Division I ECCS system (LPCS and LPCI A) pumps following a LOSP. Because of this, the Division I battery only affects those sequences in which there is a LOSP. In this analysis, only those sequences in which a LOSP had occurred were requantified. To perform this analysis, the fault tree logic for the Division I battery and EDG I were set to failed (availability set 
to 0 ), and all other equipment maintenance events were removed from the fault trees. No other sequences from the base case are affected by the Division I battery being out for scheduled maintenance, i.e., a review of the cut sets showed no Division I battery failures contributing to core damage.

The net effect of having the Division I Battery in scheduled maintenance is the same as having EDG I in scheduled maintenance, given a LOSP.

BAT-II: $\quad$ The Division II Battery (backed up by battery chargers) is required to start the Division II diesel generator and the Division II ECCS system (LPCI Trains A and B) pumps following a LOSP. Because of this, the Division II battery only affects those sequences in which there is a LOSP. In this analysis, only those sequences in which a LOSP had occurred were requantified. To perform this analysis, the fault tree logic for the Division II battery and EDG II were set to failed (availability set to 0 ), and all other equipment maintenance events were removed from the fault trees. No other sequences from the base case are affected by the Division II battery being out for scheduled maintenance, i.e., a review of the cut sets showed no Division II battery failures contributing to core damage.

The net effect of having the Division II Battery in scheduled maintenance is the same as having EDG II in scheduled maintenance, given a LOSP.

BAT-III: The Division III Battery (backed up by battery chargers) is required to start the Division III diesel generator and the HPCS pump following a LOSP. Because of this, the Division III battery only affects those sequences in which there is a LOSP followed by failure of Divisions I and II. In this analysis, only those sequences in which a LOSP had occurred followed by failure of Divisions I and II diesels were requantified. To perform this analysis, the fault tree logic for the Division III battery (HPCS) was set to failed (availability set to 0 ), and all other equipment maintenance events were removed from the fault trees. No other sequences from the base case are affected by the Division III battery being out for scheduled maintenance.

The net effect of having the Division III Battery in scheduled maintenance is the same as having EDG III in scheduled maintenance, given a LOSP.

ISSP: The Suppression Pool provides the suction source for all ECCS and also acts as a heat sink for the removal of decay heat from the core. It is not expected that the suppression pool would ever be completely drained for maintenance; however, this analysis was done to determine the vulnerability of the plant should it be drained. Those sequences in the base case where the suppression pool is empty and/or where ECCS systems are asked were requantified with the ECCS systems (i.e., HPCS, LPCS, and LPCI) set to failed (availability set to 0 ). All other equipment maintenance events were removed from the fault trees.

RHR A: $\quad$ RHR Train A performs multiple functions which include LPCI, SDC, CS, and SPC. In this analysis, RHR Train A is in scheduled maintenance, and all other systems are available. Only those sequences in the base case where RHR Train A functions are asked were requantified. To perform this analysis, the fault tree logic for the various RHR Train A functions was set to failed (availability set to 0 ), and all other equipment maintenance events were removed from the fault trees. With RHR Train A in scheduled maintenance, no other systems are made unavailable.

RHR C: $\quad$ RHR Train C performs but one function, unlike RHR Train A, which is LPCI for level control and core cooling. In this analysis, RHR Train C is in scheduled maintenance, and all other systems are available. Only those sequences in the base case where RHR Train C is asked were requantified. To perform this analysis, the fault tree logic for RHR Train C was set to failed (availability set to 0 ), and all other equipment maintenance events were removed from the fault trees. With RHR Train C in scheduled maintenance, no other systems are made unavailable. 
Appendix A

SSW A: $\quad$ SSW Train A is a support system which provides cooling water to RHR Train A, LPCS, and EDG I (see the discussion of RHR Train A above). LPCS is used for level control and core cooling in the POS 7 analysis, while EDG I is required to operate following a LOSP to power Division A components. In this analysis, RHR Train A, LPCS, and EDG I are in scheduled maintenance, and all other systems available. Only those sequences in the base case where RHR Train A functions, LPCS, and/or EDG I are asked were requantified. To perform this analysis, the fault tree logic for RHR Train A, LPCS, and EDG I was set to failed (availability set to 0 ), and all other equipment maintenance events were removed from the fault trees.

LPCS: $\quad$ LPCS is an ECCS system used for level control and core cooling in the POS 7 analysis. In this analysis, LPCS is in scheduled maintenance, and all other systems are available. Only those sequences in the base case where LPCS is asked were requantified. To perform this analysis, the fault tree logic for LPCS was set to failed (availability set to 0 ), and all other equipment maintenance events were removed from the fault trees. With LPCS in scheduled maintenance, no other systems are made unavailable.

The results of the single train maintenance analysis for POS 7 are given in Table A.2.

\section{A.1.3 Multiple Train Maintenance Analysis}

This analysis evaluated the sensitivity of the plant to multiple systems/subsystems being in scheduled maintenance while all other systems/subsystems are available (i.e., known not to be unavailable due to maintenance). During scheduled maintenance, the equipment that is scheduled to be down is out of service and standby equipment that is not scheduled to be out of service is not down, i.e., the equipment that is in standby cannot be in maintenance randomly.

The methodology and the models used to perform the multiple train maintenance analysis are the same as that used to perform the single train maintenance analysis (see Sections A.1.1 and A.1.2). The multiple train maintenance analysis results are provided in Table A.3 for POSs $0,1,2$, and 3, and Table A.4 for POSs 4, 5, 6, and 7. These table provide a listing of the combinations of systems considered out for scheduled maintenance and the point estimates for each of the combinations. 
Table A.1. Single Train Maintenance CDF Results for POSs 0, 1, 2, and 3.

\begin{tabular}{|c|c|c|c|c|}
\hline Case & Description & $\begin{array}{c}\text { POS } 0 \\
\text { Point } \\
\text { Estimate } \\
\end{array}$ & $\begin{array}{c}\text { POS 1 } \\
\text { Point } \\
\text { Estimate }\end{array}$ & $\begin{array}{l}\text { POS } 2 / 3 \\
\text { Point } \\
\text { Estimate }\end{array}$ \\
\hline Baseline & Base Case, all systems available to mitigate the accident. & $7.210 \mathrm{E}-06$ & $5.552 \mathrm{E}-06$ & 8.633E-06 \\
\hline EDG III & Emergency Diesel Generator III (HPCS) in scheduled maintenance. & $3.991 \mathrm{E}-05$ & 2.844E-05 & $5.010 \mathrm{E}-05$ \\
\hline CRD B & Control Rod Drive Train B in Scheduled Maintenance. & $7.210 \mathrm{E}-06$ & $5.552 \mathrm{E}-06$ & 8.633E-06 \\
\hline EDG I & Emergency Diesel Generator I in scheduled maintenance. & 2.831E-05 & $2.032 \mathrm{E}-05$ & 3.384E-05 \\
\hline EDG II & Emergency Diesel Generator II in scheduled maintenance. & $4.842 \mathrm{E}-05$ & $3.440 \mathrm{E}-05$ & $6.100 \mathrm{E}-05$ \\
\hline SSW C & Standby Service Water Train C (HPCS) in scheduled maintenance. & $4.856 \mathrm{E}-05$ & $3.710 \mathrm{E}-05$ & 5.557E-05 \\
\hline RHR A & Residual Heat Removal System Train A in scheduled maintenance. & $7.649 \mathrm{E}-06$ & 5.991E-06 & $9.042 \mathrm{E}-06$ \\
\hline RHR C & Residual Heat Removal System Train C in scheduled maintenance. & $7.210 \mathrm{E}-06$ & 5.552E-06 & 8.633E-06 \\
\hline SSW A & Standby Service water Train A in scheduled maintenance. & $2.983 \mathrm{E}-05$ & 2.243E-05 & 3.637E-05 \\
\hline ADS & Automatic Depressurization System in scheduled maintenance. & 9.655E-06 & $7.710 \mathrm{E}-06$ & $1.136 \mathrm{E}-05$ \\
\hline Battery I & Division I battery in scheduled maintenance. & 9.792E-05 & $6.905 \mathrm{E}-05$ & 1.219E-04 \\
\hline Battery II & Division II battery in scheduled maintenance. & $1.472 \mathrm{E}-04$ & $1.036 \mathrm{E}-04$ & 1.835E-04 \\
\hline Battery III & Division III battery in scheduled maintenance. & $3.991 \mathrm{E}-05$ & 2.844E-05 & $5.010 \mathrm{E}-05$ \\
\hline HPCS & High Pressure Core Spray in scheduled maintenance. & $1.586 \mathrm{E}-05$ & $1.421 \mathrm{E}-05$ & 1.410E-05 \\
\hline RCIC & Reactor Core Isolation Cooling in scheduled maintenance. & $2.283 \mathrm{E}-05$ & 1.627E-05 & 2.831E-05 \\
\hline LPCS & Low Pressure Core Spray in scheduled maintenance. & $9.009 \mathrm{E}-06$ & 7.350E-06 & $1.043 \mathrm{E}-05$ \\
\hline
\end{tabular}


Table A.2. Single Train Maintenance CDF Results for POSs 4, 5, 6, and 7.

\begin{tabular}{|c|c|c|c|c|c|}
\hline Case & Description & $\begin{array}{c}\text { POS } 4 \\
\text { Point } \\
\text { Estimate } \\
\end{array}$ & $\begin{array}{c}\text { POS } 5 \\
\text { Point } \\
\text { Estimate } \\
\end{array}$ & $\begin{array}{c}\text { POS } 6 \\
\text { Point } \\
\text { Estimate }\end{array}$ & $\begin{array}{c}\text { POS } 7 \\
\text { Point } \\
\text { Estimate } \\
\end{array}$ \\
\hline Baseline $\left(C_{0}\right)$ & $\begin{array}{l}\text { Baseline case, all systems available to mitigate the } \\
\text { accident. }\end{array}$ & 4.062E-05 & 1.245E-04 & $2.120 \mathrm{E}-05$ & $1.656 \mathrm{E}-05$ \\
\hline EDG III & $\begin{array}{l}\text { Emergency Diesel Generator III (HPCS) in scheduled } \\
\text { maintenance. }\end{array}$ & 9.499E-05 & 1.738E-04 & 2.207E-05 & $1.656 \mathrm{E}-05$ \\
\hline CDS & Condensate system in scheduled maintenance. & 4.062E-05 & $1.245 \mathrm{E}-04$ & $2.206 \mathrm{E}-05$ & $1.656 \mathrm{E}-05$ \\
\hline CRD B & Control Rod Drive Train B in scheduled maintenance. & $4.218 \mathrm{E}-05$ & $1.287 \mathrm{E}-04$ & 2.121E-05 & $1.656 \mathrm{E}-05$ \\
\hline EDG I & $\begin{array}{l}\text { Emergency Diesel Generator I in scheduled } \\
\text { maintenance. }\end{array}$ & $1.662 \mathrm{E}-04$ & 3.099E-04 & 2.188E-05 & $1.656 \mathrm{E}-05$ \\
\hline EDG II & $\begin{array}{l}\text { Emergency Diesel Generator II in scheduled } \\
\text { maintenance. }\end{array}$ & $1.662 \mathrm{E}-04$ & 3.087E-04 & $2.184 \mathrm{E}-05$ & $1.656 \mathrm{E}-05$ \\
\hline SSW C & $\begin{array}{l}\text { Standby Service Water Train C (HPCS) in scheduled } \\
\text { maintenance. }\end{array}$ & 3.517E-04 & 4.611E-04 & 2.661E-05 & $2.194 \mathrm{E}-05$ \\
\hline ISSP & Suppression Pool in scheduled maintenance (empty). & $8.456 \mathrm{E}-03$ & $2.008 \mathrm{E}-02$ & 2.104E-03 & 4.620E-04 \\
\hline RHR A & $\begin{array}{l}\text { Residual Heat Removal System Train A in scheduled } \\
\text { maintenance. }\end{array}$ & $4.281 \mathrm{E}-05$ & 1.277E-04 & $3.244 \mathrm{E}-05$ & $1.962 \mathrm{E}-05$ \\
\hline RHR C & $\begin{array}{l}\text { Residual Heat Removal System Train C in scheduled } \\
\text { maintenance. }\end{array}$ & 4.220E-05 & $1.271 \mathrm{E}-04$ & $2.120 \mathrm{E}-05$ & $1.676 \mathrm{E}-05$ \\
\hline SSW A & $\begin{array}{l}\text { Standby Service Water System Train A in scheduled } \\
\text { maintenance. }\end{array}$ & $1.799 \mathrm{E}-04$ & 4.145E-04 & $4.530 \mathrm{E}-05$ & $2.920 \mathrm{E}-05$ \\
\hline SRVS & All Safety Relief Valves in scheduled maintenance. & $1.644 \mathrm{E}-02$ & $1.896 \mathrm{E}-02$ & N/A & N/A \\
\hline BATTERY I & Division I Battery in scheduled maintenance. & $1.662 \mathrm{E}-04$ & 3.099E-04 & $2.188 \mathrm{E}-05$ & $1.656 \mathrm{E}-05$ \\
\hline BATTERY II & Division II Battery in scheduled maintenance. & $1.662 \mathrm{E}-04$ & $3.087 \mathrm{E}-04$ & $2.184 \mathrm{E}-05$ & $1.656 \mathrm{E}-05$ \\
\hline BATTERY III & Division III Battery (HPCS) in scheduled maintenance. & 9.499E-05 & $1.738 \mathrm{E}-04$ & 2.207E-05 & $1.656 \mathrm{E}-05$ \\
\hline HPCS & High Pressure Core Spray in scheduled maintenance. & $3.412 \mathrm{E}-04$ & 4.467E-04 & $2.663 \mathrm{E}-05$ & 2.194E-05 \\
\hline LPCS & Low Pressure Core Spray in scheduled maintenance. & $3.988 \mathrm{E}-05$ & $1.333 \mathrm{E}-04$ & 2.165E-05 & $1.699 \mathrm{E}-05$ \\
\hline
\end{tabular}


Table A.3. Multiple Train Maintenance CDF Results for POSs 0, 1, 2, and 3.

\begin{tabular}{|c|c|c|c|c|}
\hline Case & Description & $\begin{array}{c}\text { POS 0 } \\
\text { Point } \\
\text { Estimate }\end{array}$ & $\begin{array}{c}\text { POS } 1 \\
\text { Point } \\
\text { Estimate }\end{array}$ & $\begin{array}{c}\text { POS 2/3 } \\
\text { Point } \\
\text { Estimate }\end{array}$ \\
\hline DM-1 & $\begin{array}{l}\text { Emergency Diesel Generator (EDG I) } 11 \text { and Reactor Core Isolation } \\
\text { Cooling (RCIC) in scheduled maintenance. }\end{array}$ & 9.717E-05 & $6.830 \mathrm{E}-05$ & $1.212 \mathrm{E}-04$ \\
\hline DM-2 & $\begin{array}{l}\text { Emergency Diesel Generator (EDG II) } 12 \text { and Reactor Core Isolation } \\
\text { Cooling (RCIC) in scheduled maintenance. }\end{array}$ & $1.465 \mathrm{E}-04$ & $1.028 \mathrm{E}-04$ & $1.827 \mathrm{E}-04$ \\
\hline DM-3 & $\begin{array}{l}\text { Emergency Diesel Generator (EDG III) } 13 \text { and Reactor Core Isolation } \\
\text { Cooling (RCIC) in scheduled maintenance. }\end{array}$ & $1.398 \mathrm{E}-04$ & $9.815 E-05$ & $1.744 \mathrm{E}-04$ \\
\hline DM-4 & $\begin{array}{l}\text { Standby Service Water (SSW) Train C and Reactor Core Isolation Cooling } \\
\text { (RCIC) in scheduled maintenance. }\end{array}$ & $1.489 \mathrm{E}-04$ & $1.073 \mathrm{E}-04$ & $1.804 \mathrm{E}-04$ \\
\hline DM-5 & $\begin{array}{l}\text { High Pressure Core Spray (HPCS) and Reactor Core Isolation Cooling } \\
\text { (RCIC) in scheduled maintenance. }\end{array}$ & $3.197 \mathrm{E}-05$ & $2.540 \mathrm{E}-05$ & $3.426 \mathrm{E}-05$ \\
\hline DM 6 & $\begin{array}{l}\text { Control Rod Drive (CRD) Train B and Reactor Core Isolation Cooling } \\
\text { (RCIC) in scheduled maintenance. }\end{array}$ & $2.283 \mathrm{E}-05$ & $1.626 \mathrm{E}-05$ & $2.831 \mathrm{E}-05$ \\
\hline DM-7 & $\begin{array}{l}\text { Automatic Depressurization System (ADS) and Reactor Core Isolation } \\
\text { Cooling (RCIC) in scheduled maintenance. }\end{array}$ & 3.695E-05 & $3.038 \mathrm{E}-05$ & 2.243E-05 \\
\hline DM-8 & $\begin{array}{l}\text { Automatic Depressurization System (ADS) and High Pressure Core Spray } \\
\text { (HPCS) in scheduled maintenance. }\end{array}$ & 4.997E-05 & $4.800 \mathrm{E}-05$ & $4.851 \mathrm{E}-05$ \\
\hline DM-9 & Firewater system (FWS) injection capability in scheduled maintenance. & 1.194E-04 & $1.171 \mathrm{E}-04$ & $1.215 \mathrm{E}-04$ \\
\hline DM-10 & $\begin{array}{l}\text { Emergency Diesel Generator (EDG I) } 11 \text { and Emergency Diesel Generator } \\
\text { (EDG II) } 12 \text { in scheduled maintenance. }\end{array}$ & $3.166 \mathrm{E}-\mathrm{C} 4$ & 2.222E-04 & $4.000 \mathrm{E}-04$ \\
\hline DM-11 & $\begin{array}{l}\text { Emergency Diesel Generator (EDG I) } 11 \text { and Emergency Diesel Generator } \\
\text { (EDG III) } 13 \text { in scheduled maintenance. }\end{array}$ & 3.612E-04 & 2.534E-04 & 4.552E-04 \\
\hline DM-12 & Divisions I and II Batteries in scheduled maintenance. & $1.139 \mathrm{E}-03$ & $7.978 \mathrm{E}-04$ & $1.421 \mathrm{E}-03$ \\
\hline DM-13 & Divisions I and III Batteries in scheduled maintenance. & $1.322 \mathrm{E}-03$ & 9.261E-04 & $1.650 \mathrm{E}-03$ \\
\hline DM-14 & $\begin{array}{l}\text { Standby Service Water (SSW) Train A and High Pressure Core Spray } \\
\text { (HPCS) in scheduled maintenance. }\end{array}$ & $3.056 \mathrm{E}-03$ & $3.049 \mathrm{E}-03$ & $3.060 \mathrm{E}-03$ \\
\hline DM-15 & $\begin{array}{l}\text { Residual Heat Removal (RHR) Train A and Low Pressure Core Spray } \\
\text { (LPCS) in scheduled maintenance. }\end{array}$ & $9.965 \mathrm{E}-05$ & $9.800 \mathrm{E}-05$ & $1.011 E-04$ \\
\hline
\end{tabular}




\begin{tabular}{||c|l|c|c|c||}
\hline \hline Case & \multicolumn{1}{|c|}{ Description } & $\begin{array}{c}\text { POS } 0 \\
\text { Point } \\
\text { Estimate }\end{array}$ & $\begin{array}{c}\text { POS 1 } \\
\text { Point } \\
\text { Estimate }\end{array}$ & $\begin{array}{c}\text { POS 2/3 } \\
\text { Point } \\
\text { Estimate }\end{array}$ \\
\hline \hline DM-16 & $\begin{array}{l}\text { Low Pressure Core Spray (LPCS) and High Pressure Core Spray (HPCS) } \\
\text { in scheduled maintenance. }\end{array}$ & $5.287 \mathrm{E}-\mathrm{C} 5$ & $5.121 \mathrm{E}-05$ & $5.110 \mathrm{E}-05$ \\
\hline DM-17 & $\begin{array}{l}\text { Low Pressure Core Spray (LPCS) and Reactor Core Isolation Cooling } \\
\text { (RCIC) in scheduled maintenance. }\end{array}$ & $2.517 \mathrm{E}-05$ & $1.860 \mathrm{E}-05$ & $3.065 \mathrm{E}-05$ \\
\hline DM-18 & $\begin{array}{l}\text { Residual Heat Removal (RHR) Train A and High Pressure Core Spray } \\
\text { (HPCS) in scheduled maintenance. }\end{array}$ & $6.066 \mathrm{E}-05$ & $5.900 \mathrm{E}-05$ & $5.494 \mathrm{E}-05$ \\
\hline DM-19 & $\begin{array}{l}\text { Standby Service Water (SSW) Train A and Standby Service Water (SSW) } \\
\text { Train C in scheduled maintenance. }\end{array}$ & $3.423 \mathrm{E}-03$ & $3.306 \mathrm{E}-03$ & $3.522 \mathrm{E}-03$ \\
\hline DM-20 & $\begin{array}{l}\text { Residual Heat Removal (RHR) Train A and Residual Heat Removal (RHR) } \\
\text { Train C in scheduled maintenance. }\end{array}$ & $7.649 \mathrm{E}-06$ & $5.991 \mathrm{E}-06$ & $9.042 \mathrm{E}-06$ \\
\hline 1.00 & $\begin{array}{l}\text { Standby Service Water (SSW) Train A and Standby Service Water (SSW) } \\
\text { Train B in scheduled maintenance. }\end{array}$ & $6.009 \mathrm{E}-04$ & $5.064 \mathrm{E}-04$ & $6.832 \mathrm{E}-04$ \\
\hline $\begin{array}{l}\text { Desidual Heat Removal (RHR) Train A and Residual Heat Removal (RHR) } \\
\text { Train B in scheduled maintenance. }\end{array}$ & $7.649 \mathrm{E}-06$ & $5.991 \mathrm{E}-06$ & $9.042 \mathrm{E}-06$ \\
\hline
\end{tabular}


Table A.4. Multiple Train Maintenance CDF Results for POSs 4, 5, 6, and 7.

\begin{tabular}{||c|l|c|c|c|c||}
\hline Case & \multicolumn{1}{|c|}{ Description } & $\begin{array}{c}\text { POS } 4 \\
\text { Point } \\
\text { Estimate }\end{array}$ & $\begin{array}{c}\text { POS } 5 \\
\text { Point }\end{array}$ & $\begin{array}{c}\text { POS } 6 \\
\text { Point } \\
\text { Estimate }\end{array}$ & $\begin{array}{c}\text { POS 7 } \\
\text { Point } \\
\text { Estimate }\end{array}$ \\
\hline \hline DM-1 & SSW Train A and HPCS in scheduled maintenance. & $2.290 \mathrm{E}-03$ & $3.527 \mathrm{E}-03$ & $4.306 \mathrm{E}-05$ & $3.889 \mathrm{E}-04$ \\
\hline DM-2 & SSW Train A and CDS in scheduled maintenance. & $1.799 \mathrm{E}-04$ & $4.176 \mathrm{E}-04$ & $4.570 \mathrm{E}-05$ & $2.920 \mathrm{E}-05$ \\
\hline DM-3 & $\begin{array}{l}\text { Firewater system (all three pump trains) out for scheduled } \\
\text { maintenance. }\end{array}$ & $4.092 \mathrm{E}-05$ & $1.299 \mathrm{E}-04$ & $1.043 \mathrm{E}-04$ & $2.911 \mathrm{E}-05$ \\
\hline DM-4 & Firewater diesel driven pumps in scheduled maintenance. & $4.062 \mathrm{E}-05$ & $1.245 \mathrm{E}-04$ & $2.152 \mathrm{E}-05$ & $1.660 \mathrm{E}-05$ \\
\hline DM-5 & Emergency diesel generators I and II in scheduled maintenance. & $2.183 \mathrm{E}-03$ & $3.306 \mathrm{E}-03$ & $3.420 \mathrm{E}-05$ & $1.656 \mathrm{E}-05$ \\
\hline DM-6 & $\begin{array}{l}\text { Emergency diesel generators I and III in scheduled } \\
\text { maintenance. }\end{array}$ & $6.839 \mathrm{E}-04$ & $7.660 \mathrm{E}-04$ & $3.122 \mathrm{E}-05$ & $1.656 \mathrm{E}-05$ \\
\hline DM-7 & $\begin{array}{l}\text { Residual Heat Removal System Train A and all SRVs in } \\
\text { scheduled maintenance. }\end{array}$ & $2.494 \mathrm{E}-02$ & $2.683 \mathrm{E}-02$ & N/A & N/A \\
\hline DM-8 & Divisions I and II batteries in scheduled maintenance. & $2.492 \mathrm{E}-02$ & $2.504 \mathrm{E}-02$ & $3.420 \mathrm{E}-05$ & $1.656 \mathrm{E}-05$ \\
\hline DM-9 & $\begin{array}{l}\text { Shutdown Cooling Train A and the suppression pool in } \\
\text { scheduled maintenance. }\end{array}$ & $8.916 \mathrm{E}-03$ & $2.099 \mathrm{E}-02$ & $2.345 \mathrm{E}-03$ & $4.620 \mathrm{E}-04$ \\
\hline DM-10 & LPCS and HPCS in scheduled maintenance. & $4.395 \mathrm{E}-04$ & $6.006 \mathrm{E}-04$ & $2.142 \mathrm{E}-05$ & $4.236 \mathrm{E}-05$ \\
\hline DM-11 & LPCS and RHR Train A in scheduled maintenance. & $6.350 \mathrm{E}-05$ & $2.674 \mathrm{E}-04$ & $3.266 \mathrm{E}-05$ & $2.920 \mathrm{E}-05$ \\
\hline DM-12 & SSW Train A and SSW Train C in scheduled maintenance. & $2.290 \mathrm{E}-03$ & $3.527 \mathrm{E}-03$ & $5.240 \mathrm{E}-05$ & $3.889 \mathrm{E}-04$ \\
\hline DM-13 & RHR Train A and RHR Train C in scheduled maintenance. & $1.316 \mathrm{E}-04$ & $1.372 \mathrm{E}-04$ & $3.244 \mathrm{E}-05$ & $1.992 \mathrm{E}-05$ \\
\hline
\end{tabular}




\section{APPENDIX B}

RAW DATA FOR EVALUATING RISK IMPACT OF SURVEILLANCE TEST ACTIVITIES 
This appendix contains a discussion of the analysis used to determine the risk impact of performing surveillance tests for each system or subsystem and presents the raw data used for evaluating these risk impacts.

\section{B.1 Analysis Used to Evaluate the Risk Impact of Performing Surveillance Tests}

The determination of the risk impact of each surveillance test was carried out utilizing the same PRA models developed for the maintenance analysis (Section 3). The analysis is based on the following:

- The unavailability of the component (e.g., LPCS motoroperated pump) under consideration is equal to 0.0 immediately following the surveillance test $\left(R_{0}\right)$, and

- The unavailability of the component under consideration is equal to 1.0 immediately prior to the surveillance test $\left(\mathbf{R}_{1}\right)$.

Hence, the benefit of performing the surveillance test can be found.

Both of the quantities, $R_{0}$ and $R_{1}$, can be calculated using the PRA models developed in Section 3. The calculation of $R_{0}$ was performed by executing the following steps for each component/system(s) considered in this analysis:

1. The fault tree logic for the component of interest was given an unavailability of 0.0 . If a surveillance (e.g., HPCS flow test) tests more than one component in a system, all of the components tested were given an unavailability of 0.0 .

2. If the surveillance test also demands that support systems for the component of interest operate for successful completion of the test (e.g., HPCS flow test would require Division III AC and DC power, and SSW Train C to operate), all components necessary for the successful completion of the test were given an unavailability of 0.0 .

3. Once all of the components affected by the surveillance test were found and given an unavailability of 0.0 , all of the accident sequences affected by any of the components considered were requantified. That is, new cut sets were generated and a recovery analysis was applied. By regenerating the cut sets, problems with truncation errors were eliminated, and the calculation of the net effect of the surveillance test could be assured.
It is important to note the far reaching effects a surveillance test can have. In step 2 above, all support system components required for successful completion of a surveillance test, e.g., HPCS flow test, were given an unavailability of 0.0 . This means, in accident sequences were the system is not a required mitigating system, the affect of having just tested the system can still have a beneficial result because the supports for the system have just been tested as well. For example, in station blackout sequences, HPCS is not asked, but the HPCS diesel generator is called upon. If the HPCS pump flow test has just been completed, HPCS support systems could not fail the diesel generator, e.g., common service water elements to the HPCS pump and HPCS diesel generator do not fail.

The calculation of $R_{1}$ was performed by doing the following steps for each component/system(s) considered in this analysis:

1.

The fault tree logic for the component of interest was given an unavailability of 1.0. If a surveillance (e.g., HPCS flow test) tests more than one component in a system, all of the components tested were given an unavailability of 1.0 .

2. Once all of the components effected by the surveillance test were found and given an unavailability of 1.0 , all of the accident sequences affected by any of the components considered were requantified. That is, new cut sets were generated, and recovery analysis was applied. By regenerating the cut sets, problems with truncation errors were eliminated, and the calculation of the net effect of the surveillance test could be assured.

Note that unlike Step 2 for $R_{0}$, the support systems were generally not considered in the calculation of $R_{1}$ because many systems can be dependant on a particular support system and thus get tested more frequently than front line systems. For instance, the HPCS pump is tested quarterly. As part of the test, SSW Train C is demanded to operate, but SSW Train C gets demanded to operate on a monthly basis because the HPCS diesel is tested monthly. Therefore, support systems such as SSW Train C were considered in a separate analysis.

Also note that if the fault tree logic of a component is set to an unavailability of 1.0 in the PRA analysis, then its support system components were in effect set to an unavailability of 1.0 because of the way the fault tree logic was developed. Therefore, in step 1 above for the calculation of $R_{1}$, it was not necessary to give every component an unavailability of 
Appendix B

1.0 because this was in effect done at a higher level in the fault tree logic.

\section{B.2 Raw Data Used for Evaluating the Risk Impact of Surveillance Tests}

Tables B.1 through B.40 provide the results of the calculations for $R_{0}$ and $R_{1}$ for each component/subsystem considered in the analysis for each POS. The tables also give the frequency each component/subsystem is tested. 
Table B.1 $R_{\mathbb{1}}$ and $R_{1}$ for EDG 11 Surveillance Test (POS 0)

\begin{tabular}{|l|c|c|}
\hline \multicolumn{1}{|c|}{ Subsystem of Test } & $\mathbf{R}_{0}$ & $\mathbf{R}_{1}$ \\
\hline \hline EDG 11 (Tested Monthly) & $1.442 \mathrm{E}-07$ & $2.429 \mathrm{E}-05$ \\
\hline SSW A (Tested Monthly) & $3.253 \mathrm{E}-06$ & $2.983 \mathrm{E}-05$ \\
\hline EDG 11 Actuation (Tested 18 months) & $7.205 \mathrm{E}-06$ & $1.586 \mathrm{E}-05$ \\
\hline
\end{tabular}

Table B.2 $R_{\mathbf{n}}$ and $R_{1}$ for EDG 13 Surveillance Test (POS 0)

\begin{tabular}{|l|c|c|}
\hline \multicolumn{1}{|c|}{ Subsystem of Test } & $\mathbf{R}_{0}$ & $\mathbf{R}_{1}$ \\
\hline EDG 13 (Tested Monthly) & $1.390 \mathrm{E}-07$ & $3.601 \mathrm{E}-05$ \\
\hline SSW C (Tested Monthly) & $3.816 \mathrm{E}-06$ & $4.856 \mathrm{E}-05$ \\
\hline EDG 13 Actuation (Tested 18 months) & $7.202 \mathrm{E}-06$ & $8.242 \mathrm{E}-06$ \\
\hline
\end{tabular}

Table B.3 $\mathbf{R}_{\mathbf{1}}$ and $\mathbf{R}_{1}$ for LPCS Surveillance Test (POS 0)

\begin{tabular}{||l|c|c|}
\hline \multicolumn{1}{|c|}{ Subsystem of Test } & $\mathbf{R}_{0}$ & $\mathbf{R}_{1}$ \\
\hline \hline LPCS (Tested Quarterly) & $3.045 \mathrm{E}-06$ & $4.813 \mathrm{E}-06$ \\
\hline SSW A (Tested Monthly) & $3.253 \mathrm{E}-06$ & $2.983 \mathrm{E}-05$ \\
\hline LPCS Actuation (Tested 18 months) & $7.168 \mathrm{E}-06$ & $5.304 \mathrm{E}-05$ \\
\hline
\end{tabular}

" Shared with LPCI. 
Table B.4 $R_{0}$ and $R_{1}$ for LPCI Surveillance Test (POS 0)

\begin{tabular}{||c|c|c|}
\hline \multicolumn{1}{|c|}{ Subsystem of Test } & $\mathrm{R}_{0}$ & $\mathbf{R}_{1}$ \\
\hline \hline LPCI A (Tested Quarterly) & & $4.593 \mathrm{E}-06$ \\
\hline SSW A (Tested Monthly) & $3.045 \mathrm{E}-06$ & $2.983 \mathrm{E}-05$ \\
\hline LPCI A Actuation (Tested 18 months) & $3.253 \mathrm{E}-06$ & $5.304 \mathrm{E}-05$ \\
\hline
\end{tabular}

- Assumes all modes of RHR Train A are failed $\left(\mathbf{R}_{1}\right)$ or available $\left(\mathbf{R}_{0}\right)$.

** Shared with LPCS.

Table B.5 $R_{0}$ and $R_{1}$ for HPCS Surveillance Test (POS 0)

\begin{tabular}{||l|c|c|}
\hline \multicolumn{1}{|c|}{ Subsystem of Test } & $\mathbf{R}_{0}$ & $\mathbf{R}_{1}$ \\
\hline \hline HPCS (Tested Quarterly) & $3.106 \mathrm{E}-06$ & $1.293 \mathrm{E}-05$ \\
\hline SSW C (Tested Monthly) & $3.816 \mathrm{E}-06$ & $4.856 \mathrm{E}-05$ \\
\hline HPCS Actuation (Tested 18 months) & $7.203 \mathrm{E}-06$ & $3.463 \mathrm{E}-05$ \\
\hline
\end{tabular}

Table B.6 $R_{0}$ and $R_{1}$ for DCP Div. I and III Battery Test (POS 0)

\begin{tabular}{||l|c|c||}
\hline \multicolumn{1}{|c|}{ Subsystem of Test } & $\mathbf{R}_{0}$ & $\mathbf{R}_{1}$ \\
\hline \hline Battery A (Tested Quarterly) & $7.181 \mathrm{E}-06$ & $9.792 \mathrm{E}-05$ \\
\hline Battery C (Tested Quarterly) & $7.204 \mathrm{E}-06$ & $3.991 \mathrm{E}-05$ \\
\hline
\end{tabular}


Table B.7 $\mathbf{R}_{0}$ and $\mathbf{R}_{1}$ for RCIC Surveillance Test (POS 0)

\begin{tabular}{||c|c|c|}
\hline \multicolumn{1}{|c|}{ Subsystem of Test } & $\mathbf{R}_{0}$ & $\mathbf{R}_{1}$ \\
\hline \hline RCIC & $3.569 \mathrm{e}-06$ & $2.283 \mathrm{E}-05$ \\
\hline RCIC Actuation & $7.151 \mathrm{E}-06$ & $4.282 \mathrm{E}-05$ \\
\hline
\end{tabular}

Table B.8 $\mathbf{R}_{\mathbf{0}}$ and $\mathbf{R}_{1}$ for ADS Surveillance Test (POS 0)

\begin{tabular}{|l|c|c|}
\hline \multicolumn{1}{|c|}{ Subsystem of Test } & $\mathbf{R}_{0}$ & $\mathbf{R}_{1}$ \\
\hline \hline ADS & $7.208 \mathrm{E}-06$ & $9.655 \mathrm{E}-06$ \\
\hline ADS Actuation & $7.210 \mathrm{E}-06$ & $9.173 \mathrm{E}-06$ \\
\hline
\end{tabular}

Table B.9 $R_{0}$ and $R_{1}$ for EDG 11 Surveillance Test (POS 1)

\begin{tabular}{|l|c|c|}
\hline \multicolumn{1}{|c|}{ Subsystem of Test } & $\mathbf{R}_{0}$ & $\mathbf{R}_{1}$ \\
\hline \hline EDG 11 (Tested Monthly) & $1.442 \mathrm{E}-07$ & $1.708 \mathrm{E}-05$ \\
\hline SSW A (Tested Monthly) & $2.335 \mathrm{E}-06$ & $2.243 \mathrm{E}-05$ \\
\hline EDG 11 Actuation (Tested 18 months) & $4.881 \mathrm{E}-06$ & $1.161 \mathrm{E}-05$ \\
\hline
\end{tabular}

Table B.10 $R_{\mathbf{n}}$ and $R_{1}$ for EDG 13 Surveillance Test (POS 1)

\begin{tabular}{|l|l|l|}
\hline \multicolumn{1}{|c|}{ Subsystem of Test } & $\mathbf{R}_{0}$ & $\mathbf{R}_{\perp}$ \\
\hline \hline EDG 13 (Tested Monthly) & $1.390 \mathrm{E}-07$ & $2.528 \mathrm{E}-05$ \\
\hline SSW C (Tested Monthly) & $2.854 \mathrm{E}-06$ & $3.710 \mathrm{E}-05$ \\
\hline EDG 13 Actuation (Tested 18 months) & $5.546 \mathrm{E}-06$ & $6.274 \mathrm{E}-06$ \\
\hline
\end{tabular}


Table B.11 $R_{0}$ and $R_{1}$ for LPCS Surveillance Test (POS 1)

\begin{tabular}{|l|c|c|}
\hline \multicolumn{1}{|c|}{ Subsystem of Test } & $\mathbf{R}_{0}$ & $\mathbf{R}_{1}$ \\
\hline LPCS (Tested Quarterly) & $2.069 \mathrm{E}-06$ & $3.658 \mathrm{E}-06$ \\
\hline SSW A (Tested Monthly) & $2.335 \mathrm{E}-06$ & $2.243 \mathrm{E}-05$ \\
\hline LPCS Actuation (Tested 18 months) & $5.510 \mathrm{E}-06$ & $5.139 \mathrm{E}-05$ \\
\hline
\end{tabular}

- Shared with LPCI.

Table B.12 $\mathbf{R}_{0}$ and $\mathbf{R}_{1}$ for LPCI Surveillance Test (POS 1)

\begin{tabular}{||l|c|c|}
\hline \multicolumn{1}{|c|}{ Subsystem of Test } & $\mathrm{R}_{0}$ & $\mathbf{R}_{1}$ \\
\hline LPCI A (Tested Quarterly)* & $2.069 \mathrm{E}-06$ & $3.431 \mathrm{E}-06$ \\
\hline SSW A (Tested Monthly) & $2.335 \mathrm{E}-06$ & $2.243 \mathrm{E}-05$ \\
\hline LPCI A Actuation (Tested 18 months)** & $5.510 \mathrm{E}-06$ & $5.139 \mathrm{E}-05$ \\
\hline
\end{tabular}

* Assumes all modes of $\mathbf{R H R}$ Train $A$ are failed $\left(\mathbf{R}_{1}\right)$ or available $\left(\mathbf{R}_{0}\right)$.

- Shared with LPCS.

Table B.13 $R_{0}$ and $R_{1}$ for HPCS Surveillance Test (POS 1)

\begin{tabular}{|l|c|c|}
\hline \multicolumn{1}{|c|}{ Subsystem of Test } & $\mathbf{R}_{0}$ & $\mathbf{R}_{1}$ \\
\hline HPCS (Tested Quarterly) & $2.175 \mathrm{E}-06$ & $1.174 \mathrm{E}-05$ \\
\hline SSW C (Tested Monthly) & $2.854 \mathrm{E}-06$ & $3.710 \mathrm{E}-05$ \\
\hline HPCS Actuation (Tested 18 months) & $5.544 \mathrm{E}-06$ & $3.298 \mathrm{E}-05$ \\
\hline
\end{tabular}

Table B.14 $R_{0}$ and $R_{1}$ for DCP Div. I and III Battery Test (POS 1)

\begin{tabular}{|l|c|c|}
\hline \multicolumn{1}{|c|}{ Subsystem of Test } & $\mathbf{R}_{0}$ & $\mathbf{R}_{\mathbf{L}}$ \\
\hline Battery A (Tested Quarterly) & $5.531 \mathrm{E}-06$ & $6.905 \mathrm{E}-05$ \\
\hline Battery C (Tested Quarterly) & $5.547 \mathrm{E}-06$ & $2.844 \mathrm{E}-05$ \\
\hline
\end{tabular}


Table B.15 $R_{0}$ and $R_{1}$ for RCIC Surveillance Test (POS 1)

\begin{tabular}{|l|c|c|}
\hline \multicolumn{1}{|c|}{ Subsystem of Test } & $\mathbf{R}_{0}$ & $\mathbf{R}_{1}$ \\
\hline \hline RCIC & $2.825 \mathrm{E}-06$ & $1.627 \mathrm{E}-05$ \\
\hline RCIC Actuation & $5.509 \mathrm{E}-06$ & $3.168 \mathrm{E}-05$ \\
\hline
\end{tabular}

Table B.16 $\mathbf{R}_{0}$ and $\mathbf{R}_{1}$ for ADS Surveillance Test (POS 1)

\begin{tabular}{|l|c|c|}
\hline \multicolumn{1}{|c|}{ Subsystem of Test } & $\mathbf{R}_{0}$ & $\mathbf{R}_{1}$ \\
\hline \hline ADS & $5.550 \mathrm{E}-06$ & $7.710 \mathrm{E}-06$ \\
\hline ADS Actuation & $5.552 \mathrm{E}-06$ & $7.514 \mathrm{E}-06$ \\
\hline
\end{tabular}

Table B.17 $R_{0}$ and $R_{1}$ for EDG 11 Surveillance Test (POS 4)

\begin{tabular}{|l|c|c|}
\hline \multicolumn{1}{|c|}{ Subsystem of Test } & $\mathbf{R}_{0}$ & $\mathbf{R}_{1}$ \\
\hline \hline EDG 11 (Tested Monthly) & $2.627 \mathrm{E}-05$ & $1.661 \mathrm{E}-04$ \\
\hline SSW A (Tested Monthly) & $3.721 \mathrm{E}-05$ & $1.799 \mathrm{E}-04$ \\
\hline EDG 11 Actuation (Tested 18 months) & $4.174 \mathrm{E}-05$ & $4.739 \mathrm{E}-05$ \\
\hline
\end{tabular}

Table B.18 $R_{0}$ and $R_{1}$ for EDG 13 Surveillance Test (POS 4)

\begin{tabular}{|l|c|c|}
\hline \multicolumn{1}{|c|}{ Subsystem of Test } & $\mathrm{R}_{0}$ & $\mathbf{R}_{1}$ \\
\hline \hline EDG 13 (Tested Monthly) & $3.694 \mathrm{E}-05$ & $9.255 \mathrm{E}-05$ \\
\hline SSW C (Tested Monthly) & $3.886 \mathrm{E}-05$ & $3.517 \mathrm{E}-04$ \\
\hline EDG 13 Actuation (Tested 18 months) & $4.179 \mathrm{E}-05$ & $4.504 \mathrm{E}-05$ \\
\hline
\end{tabular}


Appendix B

Table B.19 $R_{0}$ and $R_{1}$ for LPCS Surveillance Test (POS 4)

\begin{tabular}{||l|l|c|}
\hline \multicolumn{1}{|c|}{ Subsystem of Test } & $\mathbf{R}_{0}$ & $\mathbf{R}_{1}$ \\
\hline \hline LPCS (Tested Quarterly) & $3.828 \mathrm{E}-05$ & $3.889 \mathrm{E}-05$ \\
\hline SSW A (Tested Monthly) & $3.721 \mathrm{E}-05$ & $1.799 \mathrm{E}-04$ \\
\hline LPCS Actuation (Tested 18 months)* & $3.976 \mathrm{E}-05$ & $5.509 \mathrm{E}-05$ \\
\hline
\end{tabular}

- Shared with LPCI.

Table B.20 $\mathbf{R}_{0}$ and $\mathbf{R}_{1}$ for LPCI Surveillance Test (POS 4)

\begin{tabular}{|l|c|c|}
\hline \multicolumn{1}{|c|}{ Subsystem of Test } & $\mathbf{R}_{0}$ & $\mathbf{R}_{1}$ \\
\hline \hline LPCI A (Tested Quarterly)* & $3.761 \mathrm{E}-05$ & $4.176 \mathrm{E}-05$ \\
\hline SSW A (Tested Monthly) & $3.721 \mathrm{E}-05$ & $1.799 \mathrm{E}-04$ \\
\hline LPCI A Actuation (Tested 18 months) $^{* *}$ & $3.976 \mathrm{E}-05$ & $5.509 \mathrm{E}-05$ \\
\hline
\end{tabular}

* Assumes all modes of RHR Train A are failed $\left(R_{1}\right)$ or available $\left(R_{0}\right)$.

"* Shared with LPCS.

Table B.21 $\mathbf{R}_{0}$ and $\mathbf{R}_{1}$ for HPCS Surveillance Test (POS 4)

\begin{tabular}{||l|c|c||}
\hline \multicolumn{1}{|c|}{ Subsystem of Test } & $\mathrm{R}_{0}$ & $\mathbf{R}_{1}$ \\
\hline \hline HPCS (Tested Quarterly) & $2.703 \mathrm{E}-05$ & $3.408 \mathrm{E}-04$ \\
\hline SSW C (Tested Monthly) & $3.886 \mathrm{E}-05$ & $3.517 \mathrm{E}-04$ \\
\hline HPCS Actuation (Tested 18 months) & $4.020 \mathrm{E}-05$ & $3.016 \mathrm{E}-04$ \\
\hline
\end{tabular}


Table B.22 $\mathbf{R}_{0}$ and $\mathbf{R}_{1}$ for DCP Div. I and III Battery Test (POS 4)

\begin{tabular}{|l|c|c|}
\hline \multicolumn{1}{|c|}{ Subsystem of Test } & $\mathbf{R}_{0}$ & $\mathbf{R}_{1}$ \\
\hline \hline Battery A (Tested Quarterly) & $4.062 \mathrm{E}-05$ & $1.662 \mathrm{E}-04$ \\
\hline Battery C (Tested Quarterly) & $4.062 \mathrm{E}-05$ & $9.499 \mathrm{E}-05$ \\
\hline
\end{tabular}

Table B.23 $\mathbf{R}_{\mathbf{q}}$ and $\mathbf{R}_{1}$ for EDG 11 Surveillance Test (POS 5)

\begin{tabular}{|l|c|c|}
\hline \multicolumn{1}{|c|}{ Subsystem of Test } & $\mathrm{R}_{0}$ & $\mathrm{R}_{1}$ \\
\hline \hline EDG 11 (Tested Monthly) & $1.030 \mathrm{E}-04$ & $3.089 \mathrm{E}-04$ \\
\hline SSW A (Tested Monthly) & $1.185 \mathrm{E}-04$ & $4.145 \mathrm{E}-04$ \\
\hline EDG 11 Actuation (Tested 18 months) & $1.259 \mathrm{E}-04$ & $1.336 \mathrm{E}-04$ \\
\hline
\end{tabular}

Table B.24 $R_{0}$ and $R_{1}$ for EDG 13 Surveillance Test (POS 5)

\begin{tabular}{|l|c|c|}
\hline \multicolumn{1}{|c|}{ Subsystem of Test } & $\mathbf{R}_{0}$ & $\mathbf{R}_{\mathbf{l}}$ \\
\hline \hline EDG 13 (Tested Monthly) & $1.206 \mathrm{E}-04$ & $1.710 \mathrm{E}-04$ \\
\hline SSW C (Tested Monthly) & $1.226 \mathrm{E}-04$ & $4.611 \mathrm{E}-04$ \\
\hline EDG 13 Actuation (Tested 18 months) & $1.260 \mathrm{E}-04$ & $1.292 \mathrm{E}-04$ \\
\hline
\end{tabular}

Table B.25 $\mathbf{R}_{0}$ and $\mathbf{R}_{1}$ for LPCS Surveillance Test (POS 5)

\begin{tabular}{||l|c|c|}
\hline \multicolumn{1}{|c|}{ Subsystem of Test } & $\mathbf{R}_{0}$ & $\mathbf{R}_{\mathbf{1}}$ \\
\hline \hline LPCS (Tested Quarterly) & $1.209 \mathrm{E}-04$ & $1.311 \mathrm{E}-04$ \\
\hline SSW A (Tested Monthly) & $1.185 \mathrm{E}-04$ & $4.145 \mathrm{E}-04$ \\
\hline LPCS Actuation (Tested 18 months) & $1.244 \mathrm{E}-04$ & $1.674 \mathrm{E}-04$ \\
\hline
\end{tabular}

- Shared with LPCI. 
Table B.26 $\mathbf{R}_{0}$ and $\mathbf{R}_{1}$ for LPCI Surveillance Test (POS 5)

\begin{tabular}{||l|c|c|}
\hline \multicolumn{1}{|c|}{ Subsystem of Test } & $\mathbf{R}_{0}$ & $\mathbf{R}_{1}$ \\
\hline \hline LPCI A (Tested Quarterly) & $1.193 \mathrm{E}-04$ & $1.251 \mathrm{E}-04$ \\
\hline SSW A (Tested Monthly) & $1.185 \mathrm{E}-04$ & $4.145 \mathrm{E}-04$ \\
\hline LPCI A Actuation (Tested 18 months) & $1.244 \mathrm{E}-04$ & $1.674 \mathrm{E}-04$ \\
\hline
\end{tabular}

- Assumes all modes of RHR Train A are failed $\left(R_{1}\right)$ or available $\left(R_{0}\right)$.

"* Shared with LPCS.

Table B.27 $\mathbf{R}_{0}$ and $\mathbf{R}_{1}$ for HPCS Surveillance Test (POS 5)

\begin{tabular}{|l|c|c|}
\hline \multicolumn{1}{|c|}{ Subsystem of Test } & $\mathbf{R}_{0}$ & $\mathbf{R}_{1}$ \\
\hline \hline HPCS (Tested Quarterly) & $1.103 \mathrm{E}-04$ & $4.462 \mathrm{E}-04$ \\
\hline SSW C (Tested Monthly) & $1.226 \mathrm{E}-04$ & $4.611 \mathrm{E}-04$ \\
\hline HPCS Actuation (Tested 18 months) & $1.240 \mathrm{E}-04$ & $4.169 \mathrm{E}-04$ \\
\hline
\end{tabular}

Table B.28 $\mathbf{R}_{0}$ and $R_{1}$ for DCP Div. I and III Battery Test (POS 5)

\begin{tabular}{|c|c|c|}
\hline Subsystem of Test & $\mathbf{R}_{n}$ & $\mathbf{R}_{1}$ \\
\hline \hline Battery A (Tested Quarterly) & $1.245 \mathrm{E}-04$ & $3.099 \mathrm{E}-04$ \\
\hline Battery C (Tested Quarterly) & $1.245 \mathrm{E}-04$ & $1.738 \mathrm{E}-04$ \\
\hline
\end{tabular}

Table B.29 $R_{0}$ and $R_{1}$ for EDG 11 Surveillance Test (POS 6)

\begin{tabular}{|l|c|c|}
\hline \multicolumn{1}{|c|}{ Subsystem of Test } & $\mathbf{R}_{0}$ & $\mathbf{R}_{1}$ \\
\hline \hline EDG 11 (Tested Monthly) & $2.069 \mathrm{E}-05$ & $2.161 \mathrm{E}-05$ \\
\hline SSW A (Tested Monthly) & $2.084 \mathrm{E}-05$ & $3.266 \mathrm{E}-05$ \\
\hline EDG 11 Actuation (Tested 18 months) & $2.120 \mathrm{E}-05$ & $2.170 \mathrm{E}-05$ \\
\hline
\end{tabular}


Table B.30 $R_{0}$ and $R_{1}$ for EDG 13 Surveillance Test (POS 6)

\begin{tabular}{|l|c|c|}
\hline \multicolumn{1}{|c|}{ Subsystem of Test } & $\mathbf{R}_{0}$ & $\mathbf{R}_{1}$ \\
\hline \hline EDG 13 (Tested Monthly) & $2.113 \mathrm{E}-05$ & $2.207 \mathrm{E}-05$ \\
\hline SSW C (Tested Monthly) & $2.120 \mathrm{E}-05$ & $2.217 \mathrm{E}-05$ \\
\hline EDG 13 Actuation (Tested 18 months) & $2.120 \mathrm{E}-04$ & $2.162 \mathrm{E}-05$ \\
\hline
\end{tabular}

Table B.31 $\mathbf{R}_{0}$ and $\mathbf{R}_{1}$ for LPCS Surveillance Test (POS 6)

\begin{tabular}{||l|c|c|}
\hline \multicolumn{1}{|c|}{ Subsystem of Test } & $\mathbf{R}_{0}$ & $\mathbf{R}_{1}$ \\
\hline \hline LPCS (Tested Quarterly) & $2.092 \mathrm{E}-05$ & $2.092 \mathrm{E}-05$ \\
\hline SSW A (Tested Monthly) & $2.084 \mathrm{E}-05$ & $3.266 \mathrm{E}-05$ \\
\hline LPCS Actuation (Tested 18 months) & $2.120 \mathrm{E}-05$ & $2.133 \mathrm{E}-05$ \\
\hline
\end{tabular}

* Shared with LPCI.

Table B.32 $\mathbf{R}_{0}$ and $\mathbf{R}_{1}$ for LPCI (RHR A) Surveillance Test (POS 6)

\begin{tabular}{|c|c|c|}
\hline Subsystem of Test & $\underline{\mathbf{R}_{0}}$ & $\mathbf{R}_{1}$ \\
\hline LPCI A (RHR A)(Tested Quarterly)* & 4.753E-06 & $3.229 \mathrm{E}-05$ \\
\hline SSW A (Tested Monthly) & $2.120 \mathrm{E}-05$ & $3.266 \mathrm{E}-05$ \\
\hline LPCI A Actuation (Tested 18 months)" & $2.120 \mathrm{E}-05$ & 2.133E-05 \\
\hline
\end{tabular}

* Assumes all modes of RHR Train A are failed $\left(\mathbf{R}_{1}\right)$ or available $\left(\mathbf{R}_{0}\right)$.

" Shared with LPCS.

Table B.33 $\mathbf{R}_{0}$ and $\mathbf{R}_{1}$ for HPCS Surveillance Test (POS 6$)$

\begin{tabular}{||l|l|c|}
\hline \multicolumn{1}{|c|}{ Subsystem of Test } & $\mathbf{R}_{0}$ & $\mathbf{R}_{1}$ \\
\hline HPCS (Tested Quarterly) & $2.119 \mathrm{E}-05$ & $2.129 \mathrm{E}-05$ \\
\hline SSW C (Tested Monthly) & $2.120 \mathrm{E}-05$ & $2.217 \mathrm{E}-05$ \\
\hline HPCS Actuation (Tested 18 months) & $2.120 \mathrm{E}-05$ & $2.138 \mathrm{E}-05$ \\
\hline
\end{tabular}


Table B.34 $\mathbf{R}_{\mathbf{0}}$ and $\mathbf{R}_{1}$ for DCP Div. I and III Battery Test (POS 6)

\begin{tabular}{|l|c|c|}
\hline \multicolumn{1}{|c|}{ Subsystem of Test } & $\mathbf{R}_{0}$ & $\mathbf{R}_{1}$ \\
\hline \hline Battery A (Tested Quarterly) & $2.120 \mathrm{E}-05$ & $2.188 \mathrm{E}-05$ \\
\hline Battery C (Tested Quarterly) & $2.120 \mathrm{E}-05$ & $2.207 \mathrm{E}-05$ \\
\hline
\end{tabular}

Table B.35 $R_{0}$ and $R_{1}$ for EDG 11 Surveillance Test (POS 7)

\begin{tabular}{|l|c|c|}
\hline \multicolumn{1}{|c|}{ Subsystem of Test } & $\mathbf{R}_{0}$ & $\mathbf{R}_{1}$ \\
\hline \hline EDG 11 (Tested Monthly) & $1.622 \mathrm{E}-05$ & $1.656 \mathrm{E}-05$ \\
\hline SSW A (Tested Monthly) & $1.642 \mathrm{E}-05$ & $2.920 \mathrm{E}-05$ \\
\hline EDG 11 Actuation (Tested 18 months) & $1.656 \mathrm{E}-05$ & $1.656 \mathrm{E}-05$ \\
\hline
\end{tabular}

Table B.36 $\mathbf{R}_{0}$ and $\mathbf{R}_{1}$ for EDG 13 Surveillance Test (POS 7)

\begin{tabular}{||l|c|c|}
\hline \multicolumn{1}{|c|}{ Subsystem of Test } & $\mathbf{R}_{0}$ & $\mathbf{R}_{1}$ \\
\hline \hline EDG 13 (Tested Monthly) & $1.653 \mathrm{E}-05$ & $1.656 \mathrm{E}-05$ \\
\hline SSW C (Tested Monthly) & $1.653 \mathrm{E}-05$ & $2.194 \mathrm{E}-05$ \\
\hline EDG 13 Actuation (Tested 18 months) & $1.656 \mathrm{E}-05$ & $1.656 \mathrm{E}-05$ \\
\hline
\end{tabular}

Table B.37 $\mathbf{R}_{0}$ and $\mathbf{R}_{1}$ for LPCS Surveillance Test (POS 7)

\begin{tabular}{||l|c|c|}
\hline \multicolumn{1}{|c|}{ Subsystem of Test } & $\mathbf{R}_{0}$ & $\mathbf{R}_{1}$ \\
\hline LPCS (Tested Quarterly) & $1.616 \mathrm{E}-05$ & $1.680 \mathrm{E}-05$ \\
\hline SSW A (Tested Monthly) & $1.642 \mathrm{E}-05$ & $2.920 \mathrm{E}-05$ \\
\hline LPCS Actuation (Tested 18 months) & $1.654 \mathrm{E}-05$ & $2.353 \mathrm{E}-05$ \\
\hline
\end{tabular}

* Shared with LPCI. 
Table B.38 $\mathbf{R}_{0}$ and $\mathbf{R}_{1}$ for LPCI Surveillance Test (POS 7)

\begin{tabular}{|l|c|c|}
\hline \multicolumn{1}{|c|}{ Subsystem of Test } & $\mathbf{R}_{0}$ & $\mathbf{R}_{1}$ \\
\hline \hline LPCI A (Tested Quarterly) & & $1.954 \mathrm{E}-05$ \\
\hline SSW A (Tested Monthly) & $1.588 \mathrm{E}-05$ & $2.920 \mathrm{E}-05$ \\
\hline LPCI A Actuation (Tested 18 months) & $1.642 \mathrm{E}-05$ & $2.353 \mathrm{E}-05$ \\
\hline
\end{tabular}

* Assumes all modes of RHR Train A are failed $\left(R_{1}\right)$ or available $\left(R_{0}\right)$.

** Shared with LPCS.

Table B.39 $\mathbf{R}_{0}$ and $\mathbf{R}_{1}$ for HPCS Surveillance Test (POS 7)

\begin{tabular}{||l|c|c|}
\hline \multicolumn{1}{|c|}{ Subsystem of Test } & $\mathbf{R}_{0}$ & $\mathbf{R}_{1}$ \\
\hline \hline HPCS (Tested Quarterly) & $1.620 \mathrm{E}-05$ & $2.194 \mathrm{E}-05$ \\
\hline SSW C (Tested Monthly) & $1.653 \mathrm{E}-05$ & $2.194 \mathrm{E}-05$ \\
\hline HPCS Actuation (Tested 18 months) & $1.655 \mathrm{E}-05$ & $2.056 \mathrm{E}-05$ \\
\hline
\end{tabular}

Table B.40 $R_{0}$ and $R_{1}$ for DCP Div. I and III Battery Test (POS 7)

\begin{tabular}{|c|c|c|}
\hline Subsystem of Test & $\mathrm{R}_{0}$ & $\mathbf{R}_{1}$ \\
\hline \hline Battery A (Tested Quarterly) & $1.656 \mathrm{E}-05$ & $1.656 \mathrm{E}-05$ \\
\hline Battery C (Tested Quarterly) & $1.656 \mathrm{E}-05$ & $1.656 \mathrm{E}-05$ \\
\hline
\end{tabular}




\section{APPENDIX C}

RISK IMPACT OF SINGLE AND MULTIPLE

MAINTENANCE ACTIVITIES 
Appendix $\mathrm{C}$ contains tables of the risk impact measures evaluated for both single and multiple component

unavailabilities in all eight POSs. The systems are ranked in terms of $I_{C D F}$ in decreasing order of importance. 
Table C.1. Impact of Single Component Unavailability at Power (POS 0)

\begin{tabular}{|c|c|c|c|c|c|c|}
\hline Rank & Train Name & $\begin{array}{c}\mathrm{I}_{\mathrm{CDF}} \\
(\mathrm{yr})\end{array}$ & $\begin{array}{c}\mathrm{AOT} \\
(\mathrm{Hrs})\end{array}$ & $\mathrm{I}_{\mathrm{CDP}}$ & $\begin{array}{c}\text { Maintenance Frequency } \\
(/ \mathrm{yr})\end{array}$ & $\mathrm{A}_{\mathrm{ICDF}}$ \\
\hline 1 & BATT II & $1.40 \mathrm{E}-4$ & 2 & $3.20 \mathrm{E}-8$ & 0.9 & $2.80 \mathrm{E}-8$ \\
\hline 2 & BATT I & $9.07 \mathrm{E}-5$ & 2 & $2.07 \mathrm{E}-8$ & 0.9 & $1.81 \mathrm{E}-8$ \\
\hline 3 & SSW C & $4.14 \mathrm{E}-5$ & 336 & $1.31 \mathrm{E}-6$ & 0.9 & $1.31 \mathrm{E}-6$ \\
\hline 4 & EDG II & $4.12 \mathrm{E}-5$ & 72 & $3.38 \mathrm{E}-7$ & 2.6 & $8.88 \mathrm{E}-7$ \\
\hline 5 & EDG III & $3.27 \mathrm{E}-5$ & 408 & $1.51 \mathrm{E}-6$ & 2.6 & $3.98 \mathrm{E}-6$ \\
\hline 5 & BATT III & $3.27 \mathrm{E}-5$ & 408 & $1.50 \mathrm{E}-6$ & 0.9 & $1.32 \mathrm{E}-6$ \\
\hline 7 & SSW A & $2.26 \mathrm{E}-5$ & 72 & $1.85 \mathrm{E}-7$ & 0.9 & $1.62 \mathrm{E}-7$ \\
\hline 8 & EDG I & $2.11 \mathrm{E}-5$ & 72 & $1.73 \mathrm{E}-7$ & 2.6 & $4.54 \mathrm{E}-7$ \\
\hline 9 & RCIC & $1.56 \mathrm{E}-5$ & 336 & $5.99 \mathrm{E}-7$ & 2.6 & $1.57 \mathrm{E}-6$ \\
\hline 10 & HPCS & $1.16 \mathrm{E}-5$ & 336 & $4.48 \mathrm{E}-7$ & 0.9 & $3.92 \mathrm{E}-7$ \\
\hline 11 & LPCS & $1.80 \mathrm{E}-6$ & 168 & $3.45 \mathrm{E}-8$ & 0.9 & $3.02 \mathrm{E}-8$ \\
\hline 12 & RHR A/LPCI A & $4.39 \mathrm{E}-7$ & 168 & $\varepsilon$ & $\varepsilon$ & $\varepsilon$ \\
\hline 13 & LPCI C & & 168 & $\varepsilon$ & 0.9 & $\varepsilon$ \\
\hline \hline
\end{tabular}

$\varepsilon$ indicates a negligible contribution. 
Table C.2. Impact of Multiple Component Unavailability at Power (POS 0)

\begin{tabular}{|c|c|c|c|c|c|c|}
\hline Rank & Train Name & $\begin{array}{l}\mathrm{I}_{\mathrm{CDF}} \\
(/ \mathrm{yr}) \\
\end{array}$ & $\begin{array}{l}\text { AOT } \\
\text { (Hrs) }\end{array}$ & $\mathrm{I}_{\mathrm{CDP}}$ & $\begin{array}{l}\text { Maintenance Frequency } \\
(/ \mathrm{yr})\end{array}$ & $A_{I C D F}$ \\
\hline 1 & SSW A \& $C^{1}$ & $3.42 \mathrm{E}-3$ & 72 & $2.81 \mathrm{E}-5$ & $3.50 \mathrm{E}-3$ & 9.85E-8 \\
\hline 2 & $\begin{array}{c}\text { LPCS, RHR/LPCl A, EDG I } \\
\& \mathrm{HPCS}^{1}\end{array}$ & 3.06E-3 & 72 & $2.52 \mathrm{E}-5$ & $3.50 \mathrm{E}-3$ & $7.56 \mathrm{E}-8$ \\
\hline 3 & 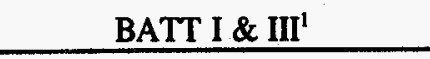 & $1.32 \mathrm{E}-3$ & 2 & $3.01 \mathrm{E}-7$ & $1.75 \mathrm{E}-3$ & $\varepsilon$ \\
\hline 4 & BATT I \& II ${ }^{1}$ & $1.14 \mathrm{E}-3$ & 2 & $2.60 \mathrm{E}-7$ & $1.75 \mathrm{E}-3$ & $\varepsilon$ \\
\hline 5 & EDG I \& III ${ }^{1}$ & $3.54 \mathrm{E}-4$ & 72 & $2.90 \mathrm{E}-6$ & 3.15E-2 & $9.14 \mathrm{E}-8$ \\
\hline 6 & EDG I \& II ${ }^{1}$ & $3.09 \mathrm{E}-4$ & 2 & $7.05 \mathrm{E}-8$ & 3.15E-2 & $\varepsilon$ \\
\hline 7 & SSW C \& RCIC & $1.41 \mathrm{E}-4$ & 336 & 4.37E-6 & $1.40 \mathrm{E}-2$ & $6.12 \mathrm{E}-8$ \\
\hline 8 & EDG II \& RCIC ${ }^{1}$ & $1.39 \mathrm{E}-4$ & 72 & $1.14 \mathrm{E}-6$ & $5.26 \mathrm{E}-1$ & $5.99 \mathrm{E}-7$ \\
\hline 9 & EDG III \& RCIC' & $1.33 \mathrm{E}-4$ & 336 & $5.08 \mathrm{E}-6$ & $5.26 \mathrm{E}-1$ & 2.67E-6 \\
\hline 10 & RHR A/LPCI A \& LPCS ${ }^{1}$ & $9.24 \mathrm{E}-5$ & 168 & $1.81 \mathrm{E}-6$ & $3.50 \mathrm{E}-3$ & $\varepsilon$ \\
\hline 11 & EDG I \& RCIC & $9.00 \mathrm{E}-5$ & 72 & 7.39E-7 & $5.26 \mathrm{E}-1$ & $3.88 \mathrm{E}-7$ \\
\hline 12 & RHR A/LPCI A \& HPCS & $5.35 \mathrm{E}-5$ & 168 & $1.03 \mathrm{E}-6$ & $3.50 \mathrm{E}-3$ & $\varepsilon$ \\
\hline 13 & LPCS \& HPCS $^{1}$ & $4.56 \mathrm{E}-5$ & 168 & 8.74E-7 & $3.50 \mathrm{E}-3$ & $\varepsilon$ \\
\hline 14 & HPCS \& RCIC & $2.48 \mathrm{E}-5$ & 336 & $9.51 \mathrm{E}-7$ & $1.40 \mathrm{E}-2$ & $1.33 \mathrm{E}-8$ \\
\hline 15 & RHR A \& $C^{*}$ & 4.39E-7 & 72 & $\varepsilon$ & $3.15 \mathrm{E}-3$ & $\varepsilon$ \\
\hline
\end{tabular}

$\varepsilon$ indicates a negligible contribution.

permitted by TSs

1 indicates that there is no TS applicable to the item. Therefore, the shortest AOT for any component in configuration is used as an upper bound. 
Appendix C

Table C.3. Impact of Single Component Unavailability at Low Power (POS 1)

\begin{tabular}{|c|c|c|c|c|}
\hline Rank & Train Name & $\begin{array}{c}\mathrm{I}_{\text {CDF }} \\
(/ \mathrm{yr})\end{array}$ & $\begin{array}{c}\text { AOT } \\
\text { (Hrs })\end{array}$ & $\mathrm{I}_{\mathrm{CDP}}$ \\
\hline 1 & BATT II & $9.80 \mathrm{E}-5$ & 2 & $2.24 \mathrm{E}-8$ \\
\hline 2 & BATT I & $6.35 \mathrm{E}-5$ & 2 & $1.45 \mathrm{E}-8$ \\
\hline 3 & SSW C & $3.15 \mathrm{E}-5$ & 336 & $1.19 \mathrm{E}-6$ \\
\hline 4 & EDG II & $2.89 \mathrm{E}-5$ & 72 & $2.37 \mathrm{E}-7$ \\
\hline 5 & EDG III & $2.29 \mathrm{E}-5$ & 408 & $1.07 \mathrm{E}-6$ \\
\hline 5 & BATT III & $2.29 \mathrm{E}-5$ & 408 & $1.07 \mathrm{E}-6$ \\
\hline 7 & SSW A & $1.69 \mathrm{E}-5$ & 72 & $1.38 \mathrm{E}-7$ \\
\hline 8 & EDG I & $1.48 \mathrm{E}-5$ & 72 & $1.21 \mathrm{E}-7$ \\
\hline 9 & RCIC & $1.07 \mathrm{E}-5$ & 336 & $4.10 \mathrm{E}-7$ \\
\hline 10 & HPCS & $8.66 \mathrm{E}-6$ & 336 & $3.32 \mathrm{E}-7$ \\
\hline 11 & ADS & $2.16 \mathrm{E}-6$ & 336 & $8.28 \mathrm{E}-8$ \\
\hline 12 & LPCS & $1.80 \mathrm{E}-6$ & 168 & $3.45 \mathrm{E}-8$ \\
\hline 13 & RHR A/LPCI A & $4.39 \mathrm{E}-7$ & 72 & $\varepsilon$ \\
\hline 13 & LPCLC & $\varepsilon$ & 72 & $\varepsilon$ \\
\hline \hline
\end{tabular}

$\varepsilon$ indicates a negligible contribution.

* indicates that there is no TS applicable to the item. 
Table C.4. Impact of Multiple Component Unavailability at Low Power (POS 1)

\begin{tabular}{|c|c|c|c|c|}
\hline Rank & Train Name & $\begin{array}{l}I_{\mathrm{CDF}} \\
(\text { /yr) }\end{array}$ & $\begin{array}{l}\text { AOT } \\
\text { (Hrs) } \\
\end{array}$ & $\mathbf{I}_{\mathrm{CDP}}$ \\
\hline 1 & SSW A \& C ${ }^{1}$ & $3.30 \mathrm{E}-3$ & 72 & 2.71E-5 \\
\hline 2 & $\begin{array}{c}\text { LPCS, RHR/LPCI A, } \\
\text { EDG I \& HPCS } \\
\end{array}$ & $3.04 \mathrm{E}-3$ & 72 & $2.50 \mathrm{E}-5$ \\
\hline 3 & BATT I \& III $^{1}$ & $9.21 \mathrm{E}-4$ & 2 & $2.10 \mathrm{E}-7$ \\
\hline 4 & BATT I \& II & $7.92 \mathrm{E}-4$ & 2 & $1.81 \mathrm{E}-7$ \\
\hline 5 & EDG I \& III & $2.48 \mathrm{E}-4$ & 72 & $2.04 \mathrm{E}-6$ \\
\hline 6 & EDG I \& II & 2.17E-4 & 2 & 4.95E-8 \\
\hline 7 & SSW C \& RCIC ${ }^{\mathbf{1}}$ & $1.02 \mathrm{E}-4$ & 336 & 3.91E-6 \\
\hline 8 & EDG II \& RCIC ${ }^{1}$ & $9.73 \mathrm{E}-5$ & 72 & 7.99E-7 \\
\hline 9 & EDG III \& RCIC ${ }^{\prime}$ & $9.26 \mathrm{E}-5$ & 336 & $3.45 \mathrm{E}-7$ \\
\hline 10 & RHR A/LPCI A \& LPCS $^{1}$ & $9.25 \mathrm{E}-5$ & 168 & $1.77 \mathrm{E}-6$ \\
\hline 11 & EDG I \& RCIC ${ }^{1}$ & $6.28 \mathrm{E}-5$ & 72 & $5.16 \mathrm{E}-7$ \\
\hline 12 & RHR A/LPCI A \& HPCS ${ }^{1}$ & $5.35 \mathrm{E}-5$ & 168 & $1.03 \mathrm{E}-6$ \\
\hline 13 & LPCS \& HPCS $^{1}$ & $4.57 \mathrm{E}-5$ & 168 & $8.76 \mathrm{E}-7$ \\
\hline 14 & ADS \& HPCS ${ }^{1}$ & $4.25 \mathrm{E}-5$ & 336 & $1.63 \mathrm{E}-6$ \\
\hline 15 & 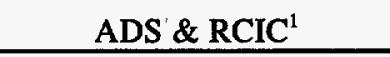 & $2.49 \mathrm{E}-5$ & 336 & $9.55 \mathrm{E}-7$ \\
\hline 16 & HPCS \& RCIC & $1.99 \mathrm{E}-5$ & 336 & $7.63 \mathrm{E}-7$ \\
\hline 17 & RHR A \& C $^{*}$ & 4.39E-7 & 72 & $\varepsilon$ \\
\hline
\end{tabular}

$\varepsilon$ indicates a negligible contribution.

* permitted by the TSs

1 indicates that there is no TS applicable to the item. Therefore, the shortest AOT for any component in configuration is used as an upper bound. 
Appendix C

Table C.5. Impact of Single Component Unavailability in POS 2 \& 3

\begin{tabular}{|c|c|c|c|c|}
\hline Rank & Train Name & $\begin{array}{c}\mathrm{I}_{\mathrm{CDF}} \\
(/ \mathrm{yr})\end{array}$ & $\begin{array}{c}\text { AOT } \\
(\mathrm{Hrs})\end{array}$ & $\mathrm{I}_{\mathrm{CDP}}$ \\
\hline 1 & BATT II & $1.75 \mathrm{E}-4$ & 24 & $4.79 \mathrm{E}-7$ \\
\hline 2 & BATT I & $1.13 \mathrm{E}-4$ & 24 & $3.90 \mathrm{E}-7$ \\
\hline 3 & EDG II & $5.24 \mathrm{E}-5$ & 24 & $1.43 \mathrm{E}-7$ \\
\hline 4 & SSW C & $4.69 \mathrm{E}-5$ & 24 & $1.28 \mathrm{E}-7$ \\
\hline 5 & EDG III & $4.15 \mathrm{E}-5$ & 24 & $1.13 \mathrm{E}-7$ \\
\hline 5 & BATT III & $4.15 \mathrm{E}-5$ & 24 & $1.13 \mathrm{E}-7$ \\
\hline 7 & SSW A & $2.77 \mathrm{E}-5$ & 24 & $7.59 \mathrm{E}-8$ \\
\hline 8 & EDG I & $2.52 \mathrm{E}-5$ & 24 & $6.90 \mathrm{E}-8$ \\
\hline 9 & RCIC & $1.97 \mathrm{E}-5$ & 24 & $5.40 \mathrm{E}-8$ \\
\hline 10 & HPCS & $5.47 \mathrm{E}-6$ & 24 & $1.50 \mathrm{E}-8$ \\
\hline 11 & ADS & $2.73 \mathrm{E}-6$ & 24 & $\varepsilon$ \\
\hline 12 & LPCS & $1.80 \mathrm{E}-6$ & 24 & $\varepsilon$ \\
\hline 13 & RHR A/LPCI A & $4.09 \mathrm{E}-7$ & 24 & $\varepsilon$ \\
\hline 14 & LPCI C & $\varepsilon$ & 24 & $\varepsilon$ \\
\hline \hline
\end{tabular}

$\varepsilon \quad$ indicates a negligible contribution.

* indicates that there is no TS applicable to the item. 
Table C.6. Impact of Multiple Component Unavailability in POS 2 \& 3

\begin{tabular}{|c|c|c|c|c|}
\hline Rank & Train Name & $\begin{array}{l}I_{\mathrm{CDF}} \\
(/ \mathrm{yr})\end{array}$ & $\begin{array}{l}\text { AOT } \\
\text { (Hrs) }\end{array}$ & $I_{C D P}$ \\
\hline 1 & SSW A \& $C^{1}$ & $3.51 \mathrm{E}-3$ & 72 & $2.89 \mathrm{E}-5$ \\
\hline 2 & $\begin{array}{c}\text { LPCS, RHR A, EDG I \& } \\
\text { HPCS }^{1}\end{array}$ & 3.05E-3 & 24 & $8.36 \mathrm{E}-6$ \\
\hline 3 & BATT I \& III $^{1}$ & $1.64 \mathrm{E}-3$ & 24 & 4.49E-6 \\
\hline 4 & BATT I \& II & $1.41 \mathrm{E}-3$ & 24 & $3.86 \mathrm{E}-6$ \\
\hline 5 & EDG I \& III & 4.47E-4 & 24 & $1.22 \mathrm{E}-6$ \\
\hline 6 & EDG I \& II & $3.91 \mathrm{E}-4$ & 24 & $1.07 \mathrm{E}-6$ \\
\hline 7 & EDG II \& RCIC' & $1.74 \mathrm{E}-4$ & 24 & 4.76E-7 \\
\hline 8 & SSW C \& RCIC' & $1.72 \mathrm{E}-4$ & 24 & 4.71E-7 \\
\hline 9 & EDG III \& RCIC' & $1.66 \mathrm{E}-4$ & 24 & 4.54E-7 \\
\hline 10 & EDG I \& RCIC & $1.13 \mathrm{E}-4$ & 24 & $3.09 \mathrm{E}-7$ \\
\hline 11 & RHR A \& LPCS ${ }^{1}$ & 9.25E-5 & 24 & $2.53 \mathrm{E}-7$ \\
\hline 12 & RHR A \& HPCS ${ }^{1}$ & $4.63 \mathrm{E}-5$ & 24 & $1.26 \mathrm{E}-7$ \\
\hline 13 & LPCS \& HPCS' & 4.25E-5 & 24 & $8.76 \mathrm{E}-7$ \\
\hline 14 & ADS \& HPCS & $3.99 \mathrm{E}-5$ & 24 & $1.90 \mathrm{E}-7$ \\
\hline 15 & HPCS \& RCIC & $2.56 \mathrm{E}-5$ & 24 & $7.01 \mathrm{E}-8$ \\
\hline 16 & CRD B \& RCIC' & $1.97 \mathrm{E}-5$ & 24 & $5.40 \mathrm{E}-8$ \\
\hline 17 & ADS \& RCIC & $1.38 \mathrm{E}-5$ & 24 & $3.78 \mathrm{E}-8$ \\
\hline 18 & RHR A \& $\mathbf{C}^{*}$ & $4.09 \mathrm{E}-7$ & 24 & $\varepsilon$ \\
\hline
\end{tabular}

indicates a negligible contribution permitted by the TSs indicates that there is no TS applicable to the item. Therefore, the shortest AOT for any component in configuration is used as an upper bound. 
Appendix C

Table C.7. Impact of Single Component Unavailability in POS 4

\begin{tabular}{|c|c|c|c|c||}
\hline Rank & Train Name & $\begin{array}{c}I_{\text {CDF }} \\
(\text { yr })\end{array}$ & $\begin{array}{c}\text { AOT } \\
(\mathrm{Hrs})\end{array}$ & $I_{\text {CDP }}$ \\
\hline 1 & SRVS & $1.64 \mathrm{E}-2$ & 24 & $4.49 \mathrm{E}-5$ \\
\hline 2 & ISSP & $8.42 \mathrm{E}-3$ & 24 & $2.31 \mathrm{E}-5$ \\
\hline 3 & SSW C & $3.11 \mathrm{E}-4$ & 24 & $8.53 \mathrm{E}-7$ \\
\hline 3 & HPCS & $3.00 \mathrm{E}-4$ & 24 & $8.21 \mathrm{E}-7$ \\
\hline 4 & SSW A & $1.39 \mathrm{E}-4$ & 24 & $7.42 \mathrm{E}-7$ \\
\hline 5 & BATT I & $1.25 \mathrm{E}-4$ & 24 & $3.42 \mathrm{E}-7$ \\
\hline 5 & EDG I & $1.25 \mathrm{E}-4$ & 24 & $3.42 \mathrm{E}-7$ \\
\hline 7 & BATT II & $1.25 \mathrm{E}-4$ & 24 & $3.42 \mathrm{E}-7$ \\
\hline 8 & EDG II & $1.25 \mathrm{E}-4$ & 24 & $3.42 \mathrm{E}-7$ \\
\hline 9 & BAT III & $5.44 \mathrm{E}-5$ & 24 & $1.49 \mathrm{E}-7$ \\
\hline 10 & EDG III & $5.44 \mathrm{E}-5$ & 24 & $1.49 \mathrm{E}-7$ \\
\hline 11 & LPCS & $4.99 \mathrm{E}-5$ & 24 & $1.36 \mathrm{E}-7$ \\
\hline 12 & RHR A & $2.19 \mathrm{E}-6$ & 24 & $\varepsilon$ \\
\hline 13 & LPCI C & $1.58 \mathrm{E}-6$ & 24 & $\varepsilon$ \\
\hline 14 & CRD B & $1.38 \mathrm{E}-6$ & $24^{* *}$ & $\varepsilon$ \\
\hline 15 & CDS & $\varepsilon 4^{* *}$ & $\varepsilon$ \\
\hline
\end{tabular}

$\varepsilon \quad$ indicates a negligible contribution.

* indicates that there is no TS applicable to the item.

** indicates that the average time spent in the POS is used as the AOT as an upper limit unless the AOT > the average time spent in the POS in which case the actual AOT is used. 
Appendix C

Table C.8. Impact of Multiple Component Unavailability in POS 4

\begin{tabular}{|c|c|c|c|c|}
\hline Rank & Train Name & $\begin{array}{l}\mathrm{I}_{\mathrm{CDF}} \\
(\mathrm{yr})\end{array}$ & $\begin{array}{l}\text { AOT } \\
\text { (Hrs) }\end{array}$ & $\mathbf{I}_{\mathrm{CDP}}$ \\
\hline 1 & RHR A \& all SRVs ${ }^{*}$ & $2.49 \mathrm{E}-2$ & $24^{a}$ & $6.82 \mathrm{E}-5$ \\
\hline 2 & BATT I \& BATT II & $2.49 \mathrm{E}-2$ & $24^{a}$ & $6.82 \mathrm{E}-5$ \\
\hline 3 & SDC A \& ISSP ${ }^{*}$ & $8.88 \mathrm{E}-3$ & $24^{a}$ & $2.43 \mathrm{E}-5$ \\
\hline 4 & SSW A \& SSW C & 2.25E-3 & $24^{\mathrm{a}}$ & $6.16 \mathrm{E}-6$ \\
\hline 5 & SSW A \& HPCS* & $2.25 \mathrm{E}-3$ & $24^{\mathrm{a}}$ & $6.17 \mathrm{E}-6$ \\
\hline 6 & EDG I \& EDG II & $2.14 \mathrm{E}-3$ & 24 & $5.86 \mathrm{E}-6$ \\
\hline 7 & EDG I \& EDG II" & $6.43 \mathrm{E}-4$ & $24^{a}$ & $1.76 \mathrm{E}-6$ \\
\hline 8 & LPCS \& HPCS ${ }^{* *}$ & $3.99 \mathrm{E}-4$ & $24^{a}$ & $1.09 \mathrm{E}-6$ \\
\hline 9 & $\begin{array}{c}\text { LPCS, RHR A \& } \\
\text { EDG I }\end{array}$ & $1.39 \mathrm{E}-4$ & $24^{a}$ & 3.80E-7 \\
\hline 10 & RHR A \& C $\mathrm{C}^{* *}$ & $9.09 \mathrm{E}-5$ & $24^{\mathrm{a}}$ & $2.49 \mathrm{E}-7$ \\
\hline 11 & RHR A \& LPCS ${ }^{* *}$ & $2.29 \mathrm{E}-5$ & 24 & $6.20 \mathrm{E}-8$ \\
\hline 12 & FWS A, B, \& C & $4.00 \mathrm{E}-7$ & $24^{a}$ & $\varepsilon$ \\
\hline 13 & FWS A \& B & $\varepsilon$ & $24^{a}$ & $\varepsilon$ \\
\hline
\end{tabular}

$\varepsilon$ indicates a negligible contribution

* indicates that there is no TS applicable to the configuration.

${ }^{11}$ permitted by the TSs

a indicates that the minimum AOT applicable to any one system within the configuration is used as long as the average time in the POS is less that the actual AOT. 
Appendix C

Table C.9. Impact of Single Component Unavailability in POS 5

\begin{tabular}{|c|c|c|c|c|}
\hline Rank & Train Name & $\begin{array}{l}I_{\mathrm{CDF}} \\
(\mathrm{yr})\end{array}$ & $\begin{array}{l}\text { AOT } \\
\text { (Hrs) }\end{array}$ & $I_{C D P}$ \\
\hline 1 & ISSP & $2.00 \mathrm{E}-2$ & ${ }^{*} 70$ & $1.60 \mathrm{E}-4$ \\
\hline 2 & SRVS* & $1.88 \mathrm{E}-2$ & 70 & $1.50 \mathrm{E}-4$ \\
\hline 3 & Ssw C & $3.37 \mathrm{E}-4$ & 336 & $1.29 \mathrm{E}-5$ \\
\hline 4 & HPCS & $3.22 \mathrm{E}-4$ & $* * 70$ & 2.57E-6 \\
\hline 5 & SSW A & $2.90 \mathrm{E}-4$ & 72 & $2.371 \mathrm{E}-6$ \\
\hline 6 & BATT I & $1.85 \mathrm{E}-4$ & 70 & $1.47 \mathrm{E}-6$ \\
\hline 7 & EDG 1 & $1.85 \mathrm{E}-4$ & 70 & $1.47 \mathrm{E}-6$ \\
\hline 8 & EDG II & $1.84 \mathrm{E}-4$ & 70 & $1.47 \mathrm{E}-6$ \\
\hline 9 & BATT II & $1.84 \mathrm{E}-4$ & $* 70$ & $1.47 \mathrm{E}-6$ \\
\hline 10 & BAT III & 4.93E-5 & 336 & $1.88 \mathrm{E}-6$ \\
\hline 11 & EDG III & 4.93E-5 & 408 & 5.88E-7 \\
\hline 12 & LPCS & $5.00 \mathrm{E}-5$ & $* 70$ & $3.97 \mathrm{E}-7$ \\
\hline 13 & CRD B & 4.20E-6 & $* 70$ & 3.35E-8 \\
\hline 14 & RHR A & $3.20 \mathrm{E}-6$ & $" 70$ & $2.56 \mathrm{E}-8$ \\
\hline 15 & LPCI C & $2.60 \mathrm{E}-6$ & $* 70$ & $2.05 \mathrm{E}-8$ \\
\hline 16 & CDS $^{*}$ & $\varepsilon$ & $* 70$ & $\varepsilon$ \\
\hline
\end{tabular}

$\varepsilon$ indicates a negligible contribution.

* indicates that there is no TS applicable to the item.

** indicates that the average time spent in the POS is used as the AOT as an upper limit. 
Table C.10. Impact of Multiple Component Unavailability in POS 5

\begin{tabular}{|c|c|c|c|c|}
\hline Rank & Train Name & $\begin{array}{c}\mathrm{I}_{\mathrm{CDF}} \\
(/ \mathrm{yr})\end{array}$ & $\begin{array}{c}\text { AOT } \\
(\mathrm{Hrs})\end{array}$ & $\mathrm{I}_{\mathrm{CDP}}$ \\
\hline 1 & RHR A \& all SRVs $^{*}$ & $2.67 \mathrm{E}-2$ & $* * 70$ & $2.13 \mathrm{E}-4$ \\
\hline 2 & BATT I \& BATT II $^{*}$ & $2.49 \mathrm{E}-2$ & ${ }^{* *} 70$ & $1.99 \mathrm{E}-4$ \\
\hline 3 & SDC A \& ISSP* $^{*}$ & $2.09 \mathrm{E}-2$ & ${ }^{* *} 70$ & $1.67 \mathrm{E}-4$ \\
\hline 4 & SSW A \& C $^{1}$ & $3.40 \mathrm{E}-3$ & ${ }^{* *} 70$ & $2.72 \mathrm{E}-5$ \\
\hline 5 & EDG I \& EDG II $^{1}$ & $3.18 \mathrm{E}-3$ & $* * 70$ & $2.54 \mathrm{E}-5$ \\
\hline 6 & SSW A \& HPCS $^{*}$ & $2.98 \mathrm{E}-3$ & $72^{\mathrm{b}}$ & $2.45 \mathrm{E}-6$ \\
\hline 7 & EDG I \& EDG III $^{*}$ & $6.42 \mathrm{E}-4$ & $408^{\mathrm{b}}$ & $2.99 \mathrm{E}-5$ \\
\hline 8 & LPCS \& HPCS $^{* * *}$ & $4.14 \mathrm{E}-4$ & $* * 70$ & $3.31 \mathrm{E}-6$ \\
\hline 9 & LPCS, RHR A, \& $^{*}$ & $2.93 \mathrm{E}-4$ & $* * 70$ & $3.31 \mathrm{E}-6$ \\
\hline 10 & RHR A \& LPCS $^{* * *}$ & $1.43 \mathrm{E}-4$ & $* * 70$ & $1.14 \mathrm{E}-6$ \\
\hline 11 & RHR A \& C & $1.27 \mathrm{E}-4$ & $* * 70$ & $1.04 \mathrm{E}-6$ \\
\hline 12 & FWS A, B, \& C & $5.40 \mathrm{E}-6$ & 24 & $2.72 \mathrm{E}-7$ \\
\hline 13 & FWS A \& B & $\varepsilon$ & 168 & $\varepsilon$ \\
\hline \hline
\end{tabular}

$\varepsilon$ indicates a negligible contribution.

* indicates that there is no TS applicable to the configuration.

** indicates that the average time spent in the POS is used as the AOT as an upper limit.

$\mathrm{b}$ indicates that the minimum AOT applicable to any one system within the configuration is used.

${ }^{* * *}$ permitted by the TSs 
Table C.11. Impact of Single Component Unavailability in POS 6

\begin{tabular}{|c|c|c|c|c|}
\hline Rank & Train Name & $\begin{array}{c}\mathrm{I}_{\text {CDF }} \\
(\text { yr) }\end{array}$ & $\begin{array}{c}\text { AOT } \\
(\mathrm{Hrs})\end{array}$ & $\mathrm{I}_{\mathrm{CDP}}$ \\
\hline 1 & ISSP & $2.08 \mathrm{E}-3$ & $114^{* *}$ & $2.71 \mathrm{E}-5$ \\
\hline 2 & SSW A & $2.41 \mathrm{E}-5$ & $72^{* *}$ & $1.98 \mathrm{E}-7$ \\
\hline 3 & RHR A & $1.12 \mathrm{E}-5$ & $114^{* *}$ & $1.45 \mathrm{E}-7$ \\
\hline 4 & EDG III & $8.70 \mathrm{E}-7$ & $408^{* *}$ & $4.04 \mathrm{E}-8$ \\
\hline 4 & BATT III & $8.70 \mathrm{E}-7$ & $336^{* *}$ & $3.33 \mathrm{E}-8$ \\
\hline 6 & CDS & $8.60 \mathrm{E}-7$ & $114^{* *}$ & $1.12 \mathrm{E}-8$ \\
\hline 7 & EDG I & $6.80 \mathrm{E}-7$ & $114^{* *}$ & $\varepsilon$ \\
\hline 7 & BATT I & $6.80 \mathrm{E}-7$ & $114^{* *}$ & $\varepsilon$ \\
\hline 9 & EDG II & $6.40 \mathrm{E}-7$ & $114^{* *}$ & $\varepsilon$ \\
\hline 9 & BATT II & $6.40 \mathrm{E}-7$ & $114^{* *}$ & $\varepsilon$ \\
\hline 11 & HPCS & $5.43 \mathrm{E}-6$ & $114^{* *}$ & $\varepsilon$ \\
\hline 12 & SSW C & $5.41 \mathrm{E}-6$ & $336^{b}$ & $2.07 \mathrm{E}-7$ \\
\hline 13 & LPCI C & $1.00 \mathrm{E}-8$ & $114^{* *}$ & $\varepsilon$ \\
\hline 14 & CRD B & $1.00 \mathrm{E}-8$ & $114^{* *}$ & $\varepsilon$ \\
\hline 15 & LPCS & $4.50 \mathrm{E}-7$ & $114^{* *}$ & $\varepsilon$ \\
\hline \hline
\end{tabular}

$\varepsilon$ indicates a negligible contribution.

* indicates that there is no TS applicable to the item.

"* indicates that the average time spent in the POS is used as the AOT as an upper limit. 
Table C.12. Impact of Multiple Component Unavailability in POS 6

\begin{tabular}{|c|c|c|c|c|}
\hline Rank & Train Name & $\begin{array}{l}I_{\mathrm{CDF}} \\
(/ \mathrm{yr})\end{array}$ & $\begin{array}{l}\text { AOT } \\
\text { (Hrs) }\end{array}$ & $\mathbf{I}_{\mathrm{CDP}}$ \\
\hline 1 & SDC A \& ISSP* & $2.32 \mathrm{E}-3$ & $114^{* *}$ & $3.00 \mathrm{E}-5$ \\
\hline 2 & FWS A, B, \& C & 8.31E-4 & $114^{* *}$ & $1.08 \mathrm{E}-5$ \\
\hline 3 & SSW A \& SSW C* & $4.02 \mathrm{E}-4$ & $114^{* *}$ & $5.23 \mathrm{E}-6$ \\
\hline 4 & $\begin{array}{c}\text { LPCS, RHR A, \& EDG I } \\
\text { CDS }^{*}\end{array}$ & $2.45 \mathrm{E}-5$ & $114^{* *}$ & $3.18 \mathrm{E}-7$ \\
\hline 5 & SSW A \& HPCS* & $2.19 \mathrm{E}-5$ & $72^{b}$ & $1.79 \mathrm{E}-7$ \\
\hline 6 & EDG I \& EDG II* & $1.30 \mathrm{E}-5$ & $114^{* *}$ & $1.69 \mathrm{E}-7$ \\
\hline 7 & BATT I \& BATT II & $1.30 \mathrm{E}-5$ & $114^{* *}$ & $1.69 \mathrm{E}-7$ \\
\hline 8 & LPCS \& RHR A ${ }^{* * * *}$ & $1.15 \mathrm{E}-5$ & $114^{* *}$ & $1.49 \mathrm{E}-7$ \\
\hline 9 & RHR A \& C C $^{* * *}$ & $1.12 \mathrm{E}-5$ & $114^{* *}$ & $1.45 \mathrm{E}-7$ \\
\hline 10 & EDG I \& EDG III & $1.00 \mathrm{E}-5$ & $408^{b}$ & 4.65E-7 \\
\hline 11 & FWS A \& B* & $3.20 \mathrm{E}-7$ & $114^{* *}$ & $\varepsilon$ \\
\hline 12 & LPCS \& HPCS ${ }^{* * *}$ & $2.20 \mathrm{E}-7$ & $114^{* *}$ & $\varepsilon$ \\
\hline
\end{tabular}

$\varepsilon$ indicates a negligible contribution.

* indicates that there is no TS applicable to the configuration.

** indicates that the average time spent in the POS is used as the AOT as an upper limit.

$b$ indicates that the minimum AOT applicable to any one system within the configuration is used.

**** permitted by the TSs 
Table C.13. Impact of Single Component Unavailability in POS 7

\begin{tabular}{|c|c|c|c|c|}
\hline Rank & Train Name & $\begin{array}{c}\mathrm{I}_{\text {CDF }} \\
(\text { yr) }\end{array}$ & $\begin{array}{c}\text { AOT } \\
(\mathrm{Hrs})\end{array}$ & $\mathrm{I}_{\mathrm{CDP}}$ \\
\hline 1 & ISSP & $4.45 \mathrm{E}-4$ & $368^{* *}$ & $1.87 \mathrm{E}-5$ \\
\hline 2 & SSW A & $1.26 \mathrm{E}-5$ & $72^{* *}$ & $1.03 \mathrm{E}-7$ \\
\hline 3 & HPCS & $5.34 \mathrm{E}-6$ & $368^{* *}$ & $2.24 \mathrm{E}-7$ \\
\hline 4 & SSW C & $5.34 \mathrm{E}-6$ & $336^{\mathrm{b}}$ & $2.04 \mathrm{E}-7$ \\
\hline 5 & RHR A & $3.04 \mathrm{E}-6$ & $368^{* *}$ & $1.27 \mathrm{E}-7$ \\
\hline 6 & LPCS & $4.40 \mathrm{E}-7$ & $368^{* *}$ & $1.85 \mathrm{E}-8$ \\
\hline 7 & LPCI C & $1.40 \mathrm{E}-7$ & $368^{* *}$ & $\varepsilon$ \\
\hline 8 & EDG I & $\varepsilon$ & $368^{* *}$ & $\varepsilon$ \\
\hline 8 & EDG II & $\varepsilon$ & $368^{* *}$ & $\varepsilon$ \\
\hline 8 & EDG III & $\varepsilon$ & $408^{\mathrm{b}}$ & $\varepsilon$ \\
\hline 8 & BATT I & $\varepsilon$ & $368^{* *}$ & $\varepsilon$ \\
\hline 8 & BATT II & $\varepsilon$ & $368^{* *}$ & $\varepsilon$ \\
\hline 8 & BATT III & $\varepsilon$ & $336^{\mathrm{b}}$ & $\varepsilon$ \\
\hline 8 & CRD B* & $\varepsilon$ & $368^{* *}$ & $\varepsilon$ \\
\hline 8 & CDS & $\varepsilon$ & $368^{* *}$ & $\varepsilon$ \\
\hline
\end{tabular}

$\varepsilon$ indicates a negligible contribution.

* indicates that there is no TS applicable to the item.

** indicates that the average time spent in the POS is used as the AOT as an upper limit. 
Table C.14. Impact of Multiple Component Unavailability in POS 7

\begin{tabular}{|c|c|c|c|c|}
\hline Rank & Train Name & $\begin{array}{l}\mathrm{I}_{\mathrm{CDF}} \\
(/ \mathrm{yr})\end{array}$ & $\begin{array}{l}\text { AOT } \\
\text { (Hrs) }\end{array}$ & $\mathbf{I}_{\mathrm{CDP}}$ \\
\hline 1 & SDC A \& ISSP" & 4.45E-4 & $368^{* *}$ & $1.87 \mathrm{E}-5$ \\
\hline 2 & SSW A \& SSW C & $3.72 \mathrm{E}-4$ & $72^{6}$ & $3.06 \mathrm{E}-6$ \\
\hline 3 & LPCS \& HPCS $^{* * * 1}$ & 2.57E-5 & $368^{* *}$ & $1.08 \mathrm{E}-6$ \\
\hline 4 & $\begin{array}{c}\text { LPCS, RHR A, \& } \\
\text { EDG I CDS }\end{array}$ & $1.26 \mathrm{E}-5$ & $368^{* *}$ & $5.29 \mathrm{E}-7$ \\
\hline 5 & LPCS \& RHR A ${ }^{* * *}$ & $1.26 \mathrm{E}-5$ & $368^{* *}$ & $5.26 \mathrm{E}-7$ \\
\hline 6 & FWS A, B, \& $\mathrm{C}^{*}$ & $1.25 \mathrm{E}-5$ & $368^{* *}$ & $5.25 \mathrm{E}-7$ \\
\hline 7 & RHR A \& C $C^{* * *}$ & $3.36 \mathrm{E}-6$ & $368^{* *}$ & $1.41 \mathrm{E}-7$ \\
\hline 8 & FWS A \& B ${ }^{*}$ & $4.00 \mathrm{E}-8$ & $368^{* *}$ & $\varepsilon$ \\
\hline 9 & EDG I \& EDG II* & $\varepsilon$ & $368^{* *}$ & $\varepsilon$ \\
\hline 9 & BATT I \& BATT II & $\varepsilon$ & $368^{* *}$ & $\varepsilon$ \\
\hline 9 & EDG I \& EDG III & $\varepsilon$ & $408^{b}$ & $\varepsilon$ \\
\hline
\end{tabular}

$\varepsilon$ indicates a negligible contribution.

* indicates that there is no TS applicable to the configuration.

" indicates that the average time spent in the POS is used as the AOT as an upper limit.

b indicates that the minimum AOT applicable to any one system within the configuration is used.

${ }^{* * *}$ permitted in the TSs 


\section{APPENDIX D}

\section{RISK IMPACT OF SURVEILLANCE}

ACTIVITIES 
Appendix D contains a table of the risk impact measures evaluated for surveillance test activities. 


\begin{tabular}{|c|c|c|c|c|c|c|c|c|c|c|c|c|c|c|c|}
\hline 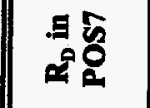 & $\omega$ & $\omega$ & $\omega$ & $\infty$ & $\omega$ & $\omega$ & $\omega$ & $\omega$ & $\omega$ & $\omega$ & $\omega$ & $\omega$ & $\omega$ & $\omega$ & $\omega$ \\
\hline$\Xi 8$ & 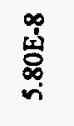 & 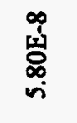 & $\omega$ & $\begin{array}{l}\infty \\
\text { ஸे } \\
\text { స్| } \\
\end{array}$ & $\omega$ & $\omega$ & $\omega$ & $\omega$ & $\omega$ & $\omega$ & 兽 & $\omega$ & $\omega$ & $\omega$ & $\omega$ \\
\hline 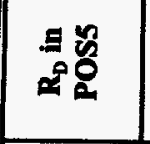 & 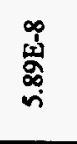 & 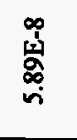 & 离 & $\omega$ & $\omega$ & مُ & 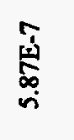 & 我 & 禾 & $\omega$ & $\omega$ & 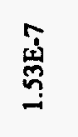 & $\omega$ & 宋 & 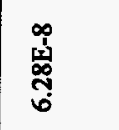 \\
\hline 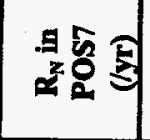 & $\omega$ & $\omega$ & $\omega$ & $\omega$ & $\omega$ & $\omega$ & $\omega$ & $\omega$ & $\omega$ & $\omega$ & $\omega$ & $\omega$ & $\omega$ & $\omega$ & $\omega$ \\
\hline 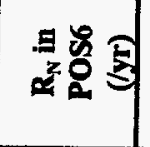 & 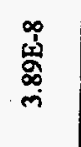 & 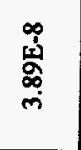 & $\omega$ & $\omega$ & $\omega$ & $\omega$ & $\omega$ & $\omega$ & $\omega$ & $\omega$ & 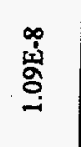 & $\omega$ & $\omega$ & $\omega$ & $\omega$ \\
\hline 프웡 & 离 & 蒿 & 盛 & 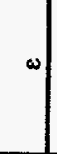 & $\omega$ & : & & 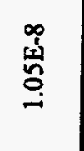 & 丞 & $\omega$ & $\omega$ & 预 & $\omega$ & 釆 & $\begin{array}{l}\stackrel{\infty}{a} \\
\stackrel{a}{\bar{a}} \\
\alpha\end{array}$ \\
\hline 용 & 踤 & 量 & 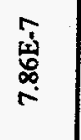 & 势 & 离 & 离 & 点 & 悹 & 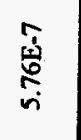 & 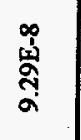 & $\begin{array}{l}\infty \\
\substack{0 \\
\mathbf{0} \\
\infty \\
\infty \\
\infty}\end{array}$ & 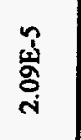 & 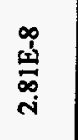 & $\begin{array}{l}\text { a } \\
\text { a } \\
\text { को }\end{array}$ & 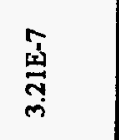 \\
\hline : & 曷 & 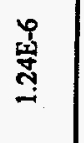 & 密 & ลे & 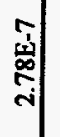 & \begin{tabular}{l} 
章 \\
0 \\
0 \\
\hdashline
\end{tabular} & 告 & 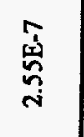 & $\begin{array}{l}\text { a } \\
\stackrel{2}{0} \\
\text { in }\end{array}$ & 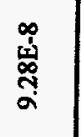 & 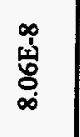 & 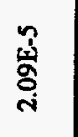 & 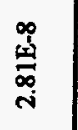 & $\begin{array}{l}\frac{3}{2} \\
\frac{2}{\alpha}\end{array}$ & 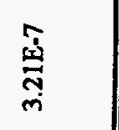 \\
\hline ₫ है & 另 & 岌 & 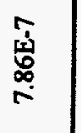 & 离 & 离 & $\begin{array}{l}1 \\
\text { âd } \\
\infty \\
\infty \\
+\end{array}$ & 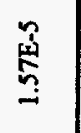 & 悹 & $\begin{array}{l}\text { 离 } \\
\stackrel{0}{\circ} \\
\dot{n}\end{array}$ & 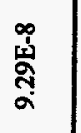 & \begin{tabular}{l}
$\infty$ \\
0 \\
\multirow{4}{0}{} \\
$\infty$ \\
$\infty$
\end{tabular} & 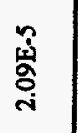 & 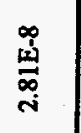 & 峁 & 离 \\
\hline 酸 & $\simeq$ & $\beth$ & $\simeq$ & $\nabla$ & $\nabla$ & $\nabla$ & $\approx$ & $a$ & $n$ & $n$ & $n$ & $\approx$ & $a$ & $\simeq$ & $\simeq$ \\
\hline 窝鄫 & $\stackrel{\infty}{-1}$ & $\stackrel{\infty}{=}$ & $\stackrel{\infty}{=}$ & $\stackrel{\infty}{-1}$ & 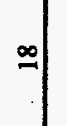 & $\infty$ & $\stackrel{\infty}{ }$ & $\stackrel{\infty}{=}$ & $\infty$ & $\stackrel{\infty}{\sim}$ & $\stackrel{\infty}{\sim}$ & $\stackrel{\infty}{=}$ & $\stackrel{\infty}{=}$ & $\stackrel{\infty}{-}$ & $\stackrel{\infty}{\rightleftharpoons}$ \\
\hline 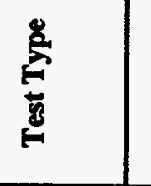 & 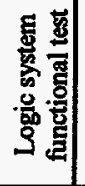 & 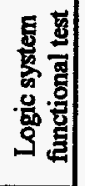 & 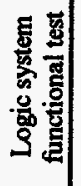 & 产 & 훌 & 空 & 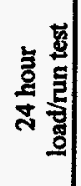 & 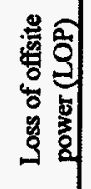 & 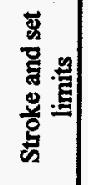 & 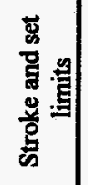 & 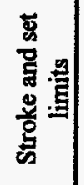 & 憘 & 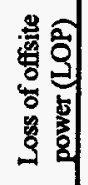 & 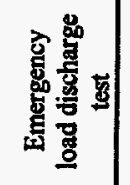 & 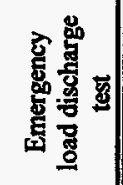 \\
\hline 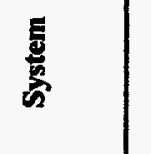 & 边 & 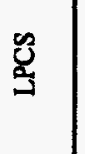 & 玹 & $\begin{array}{l}\leq \\
0 \\
0\end{array}$ & 岂 & $\begin{array}{l}0 \\
0 \\
0 \\
0\end{array}$ & $\bar{\delta}$ & 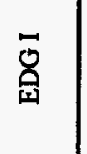 & 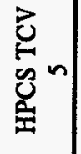 & $\begin{array}{l}3 \\
z \\
y \\
y \\
3\end{array}$ & 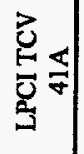 & $\begin{array}{l}\text { 范 } \\
8\end{array}$ & 目 & $\vec{E}$ & $\underset{\Xi}{E}$ \\
\hline
\end{tabular}




\section{APPENDIX E}

\section{SYSTEM DESCRIPTIONS}


This appendix contains system descriptions for the major systems analyzed in the study. The systems include the Emergency Power System (EPS), i.e., the batteries and emergency diesel generators, the Standby Service Water (SSW) System, the High Pressure Core Spray System (HPCS), the Low Pressure Core Spray System (LPCS), the Low Pressure Coolant Injection System (LPCD), the Alternate Decay Heat Removal System (ADHRS) and the Automatic Depressurization System (ADS), i.e., Safety Relief Valves (SRVs).

\section{E.1 Emergency Power System (EPS)}

\section{E.1.1 EPS System Description}

The EPS consists of the AC and DC power divisions required by all systems (except firewater) needed to mitigate postulated accidents. This includes Balance of Plant (BOP) and Engineered Safety Feature (ESF) buses. Both ESF AC and DC power are divided into three separate divisions. Two of the divisions ( 1 and 2 ) are for the majority of the ESF and the third (3) is dedicated to the HPCS system and its required support systems. The EPS schematic is shown in Figure E1.

The ESF AC divisions normally receive power from one of three offsite sources through ESF transformers ( 34.5 $\mathrm{kV} / 4.16 \mathrm{kV}$ ). In addition to the normal supply from the ESF transformers, each ESF $4.16 \mathrm{kV}$ bus has a standby diesel generator which is available to supply bus loads upon a loss of normal AC power. These diesels may be started manually or automatically. The diesels supplying Divisions 1 and 2 buses are rated at $7000 \mathrm{~kW}$ and start on a loss of normal AC power to the associated bus, low reactor level of -150 inches, or high drywell pressure of $+2 \mathrm{psig}$. The diesel supplying Division 3 buses (rated at $3300 \mathrm{~kW}$ ) is exclusively for HPCS and starts on a loss of normal AC, low reactor water level ( -42 inches), and high drywell pressure signal of +2 psig. For Divisions 1 and 2, the transfer of power from normal to backup or emergency power supplies is controlled by the load shedding and sequencing system.

For Divisions 1 and 2, when a loss of normal pówer signal occurs, the diesel generators automatically start and connect to the associated ESF bus if no other source of power is available. To prevent overloading the diesel generator when no alternate source is available, unnecessary loads are shed from the associated bus and those loads required for plant safety are sequenced onto the bus. For Division 3, when a loss of normal power occurs, the diesel generator will start and automatically close on the bus when at speed and voltage.
If Divisions 1 and 2 diesel generators fail to power their buses, power can be supplied to certain Division 1 or 2 loads from the HPCS diesel generator. This is accomplished by isolating the normal Division 3 loads from the diesel and connecting either the Division 1 or 2 loads to the HPCS diesel generator. The electrical equipment that is involved in accomplishing this is shown in Figure E2.

The ESF $125 \mathrm{~V}$ DC system includes three divisions, each consisting of two battery chargers which normally supply the load and a bank of batteries which function as a backup. Divisions 1 and 2 of the ESF DC (Buses 11DA and 11DB, respectively) system supply the majority of the ESF loads. Both are rated at 1600 amperes. Division 3 (Bus 11DC) is dedicated to the HPCS system and is rated at 100 ampere hours.

The battery chargers normally supplying power to each ESF bus are silicon controlled, rectifier type chargers rated at 400 amperes, 125 V DC. The ESF battery chargers maintain the terminal voltage of the associated batteries above a minimum of 1.75 volts per cell. Either charger can restore the batteries from this minimum voltage to their fully charged state within eight hours under normal plant operating conditions.

Each ESF DC battery bank consists of sixty lead-calcium type cells connected in series to produce the rated output of 125 V DC. Each ESF battery bank can supply the required DC loads for eleven hours after a loss of AC power if unnecessary loads are shed.

Most of the EPS is located in the diesel building and in compartmentalized rooms within the auxiliary building. Any physical impact of accident conditions on the ability of the EPS to perform its function would be minimal. Diesel generators are assumed to fail in fifteen minutes without room cooling. The battery and switchgear rooms do not require room cooling success during accident conditions.

\section{E.1.2 EPS Interfaces and Dependencies}

Each diesel generator has six subsystems required for its operation: (1) fuel oil subsystem, (2) air starting subsystem, (3) lube oil subsystem, (4) jacket water cooling subsystem, (5) combustion air intake, exhaust and crankcase ventilation, and (6) standby generator excitation subsystem. With the exception of the combustion air subsystem, all of these subsystems are normally treated as part of the diesel generator. However, some of these other subsystems are dependent on operation of other systems. The important dependencies are listed below and shown in Figure E3 with major dependencies indicated by solid diamonds. 

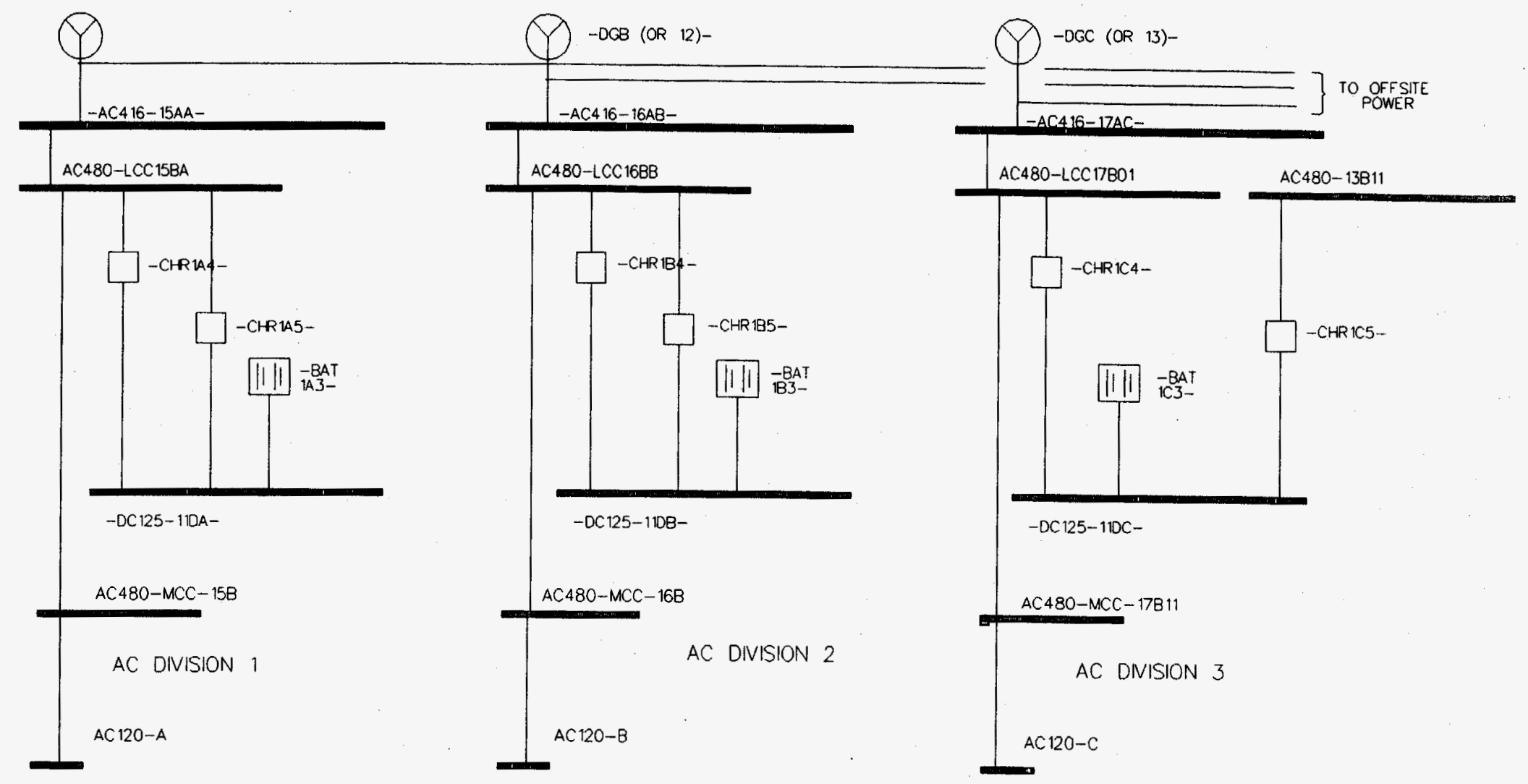

Figure E1. EPS System Schematic. 
Appendix E

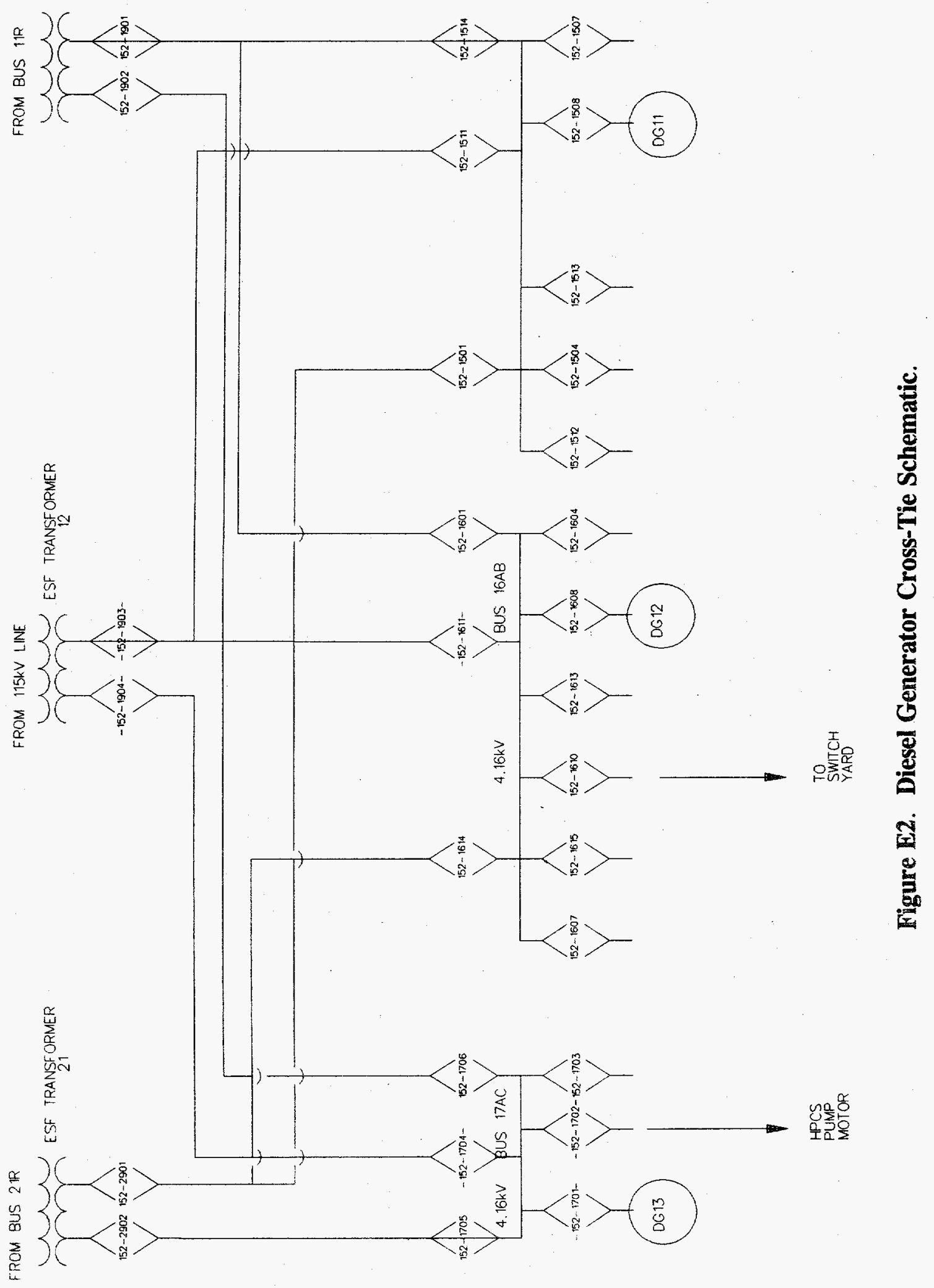




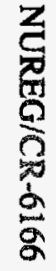

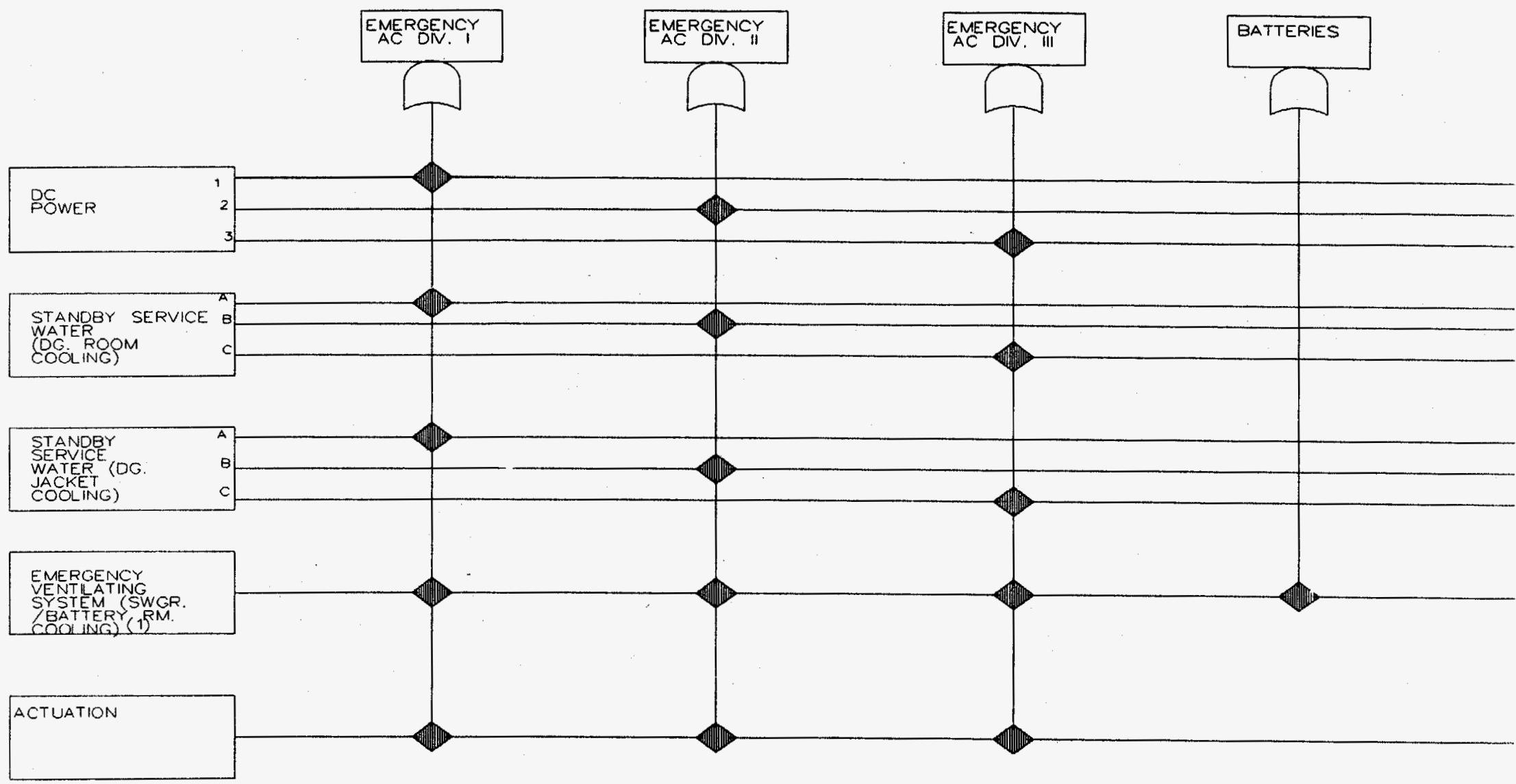

DEPENDENCY DIAGRAM IS SHOWN USING FAILURE LOGIC.

(1) DEPENDENCY NOT REQUIRED DURING SHORT TERM OPERATION.

Figure E3. EPS Dependency Diagram. 
The ESF AC divisions require DC power from the associated ESF DC buses for circuit breaker control power, diesel generator field flashing, and the diesel fuel oil booster pump. The SSW system is required to supply cooling water to the jacket water cooler.

The dependencies for Division 3 (i.e., when Division 1 or 2 loads are powered from HPCS diesel generator) are the same as those for the HPCS diesel generator (Division 3 EDG) shown in Figure E3. There is a limitation as to which loads can be supported by the Division 3 HPCS diesel generator when cross-tied to either Division 1 or 2 . The following loads on either Division 1 or 2 can be supplied by the Division 3 diesel generator:

- RHR Pump,

- SSW Pump,

- Motor-Operated Valves,

- Control Room Emergency Fan,

- Battery Charger,

- Drywell Coolers,

- Fuel Pool Cooling and Recirculation Pump,

- SSW Pump Fan,

- Control Room Air Conditioning and Fan,

- SSW Cooling Tower Fans,

- ESF Electrical Room Cooler Fan,

- Instrument Air Compressor,

- Safeguards Switchgear and Battery Room Fans,

- ECCS Pump Room Cooler, and

- HPCS Diesel Generator Accessories Cooling 60 Water Pumps,

- Supply Fan and Auxiliaries.

Room cooling is required for the diesel generator rooms. The diesel generator room cooling provides combustion air to the diesel.

\section{E.2 Standby Service Water (SSW) System}

\section{E.2.1 SSW System Description}

The function of the SSW system is to provide heat removal from plant auxiliaries that require cooling water during an emergency shutdown of the plant.

The SSW system is made up of three independent trains: A, B, and C. Each train consists of a motor-driven pump, motor-operated valves, and heat exchangers. Train $C$ is dedicated to the HPCS system.

SSW Pumps A and B are vertical, centrifugal pumps, each with a 12,000 gpm capacity. SSW Pump $C$ is also a vertical, centrifugal pump, but with only a $1300 \mathrm{gpm}$ capacity. Each pump takes water from the cooling tower basins, circulates water through the heat exchangers for each load, and returns the water to the towers through a motor-operated discharge valve. Each train has its own discharge valve. A simplified schematic of the SSW system is provided in Figures E4 and E5. Major system components are shown with valves shown in their normal standby positions.

The SSW pumps are located in pump houses away from the other buildings on the site, out by the cooling tower. Because of the relative location of the system components, local access to the SSW system would not be affected by either containment venting or failure. Each pump house normally has open louvers on the walls. This, along with the air current from the proximity of the cooling tower, was assumed to provide ample room ventilation. Thus, a loss of room cooling to the SSW pumps was not considered to fail the pumps.

The SSW system is automatically initiated and controlled. However, operator intervention is required to manually start the system given an auto-start failure.

\section{E.2.2 SSW Interfaces and Dependencies}

The SSW system major dependencies are DC control power for initiating the actuation relay logic, and $\mathrm{AC}$ power for operating the SSW pumps and valves. The pumps are self-cooled. 

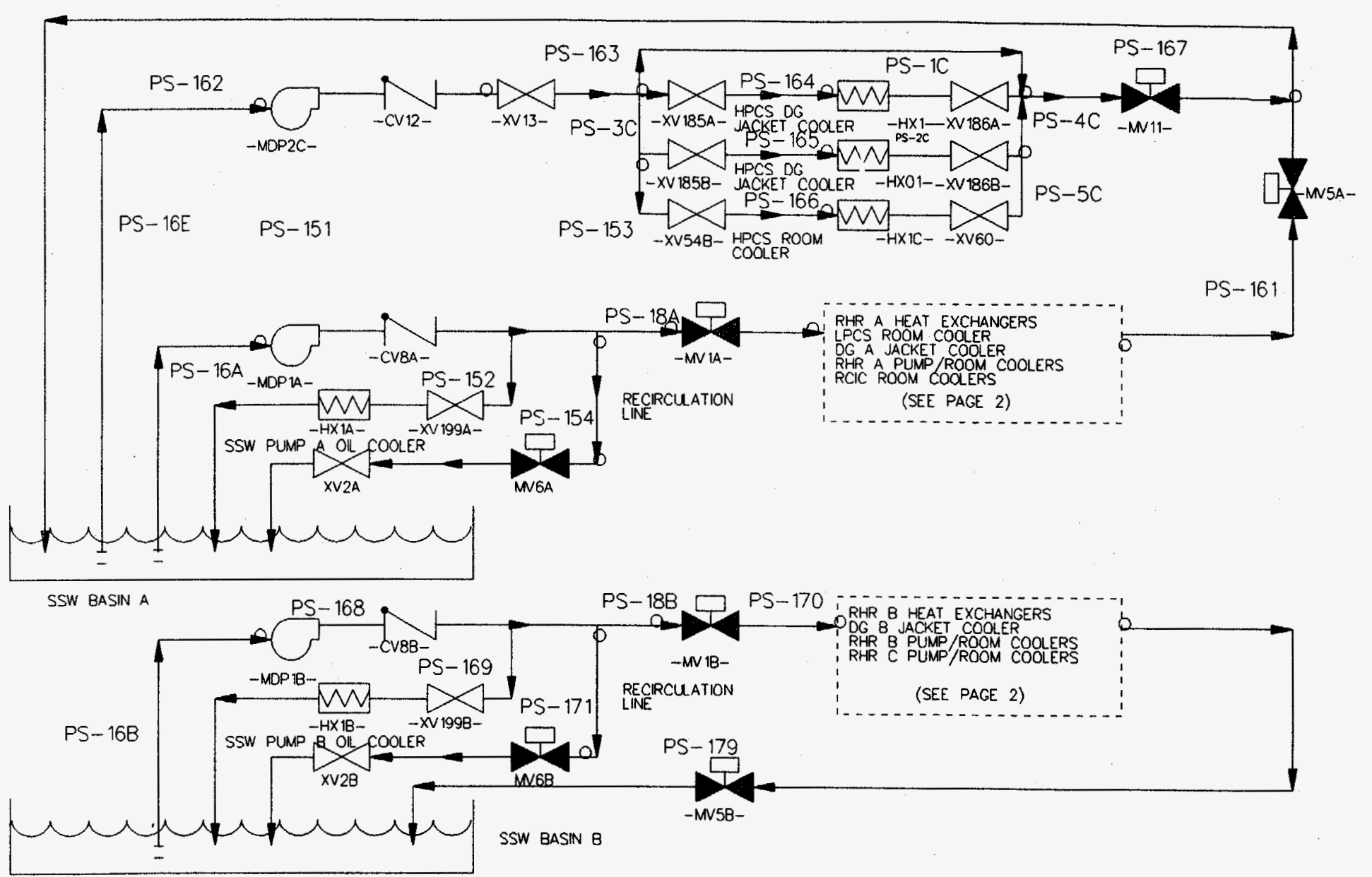

VALVE POSITIONS aRE SHOWN IN THER StandDY MODE

Figure E4. SSW System Schematic (Page 1 of 2). 


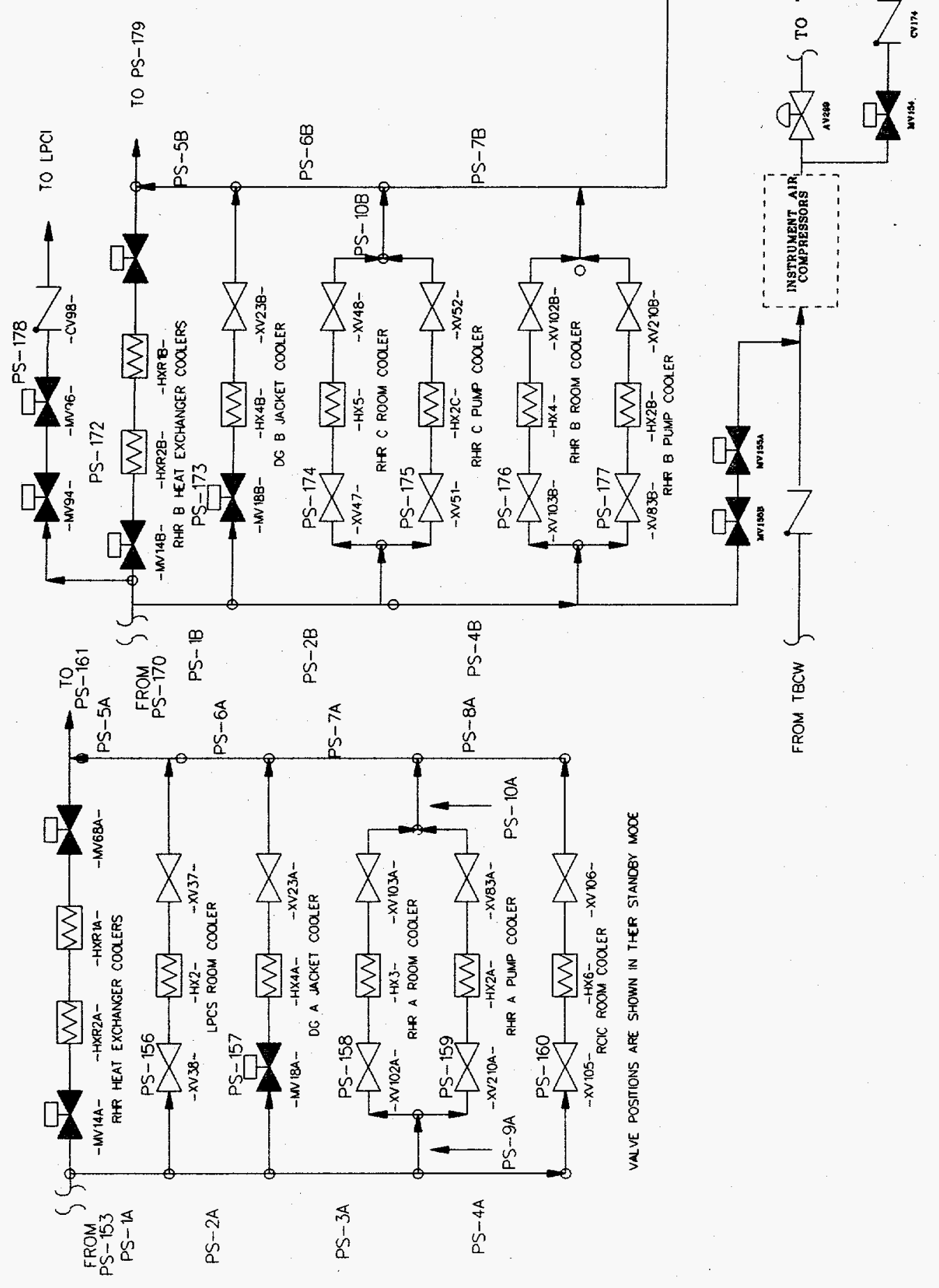

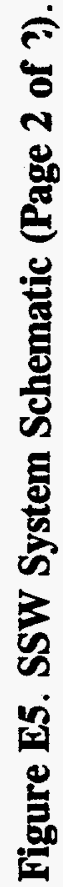


The $\mathrm{DC}$ power to Trains $\mathrm{A}, \mathrm{B}$ and $\mathrm{C}$ is provided by the Division 1125 V DC, Division 2125 V DC, and Division 3 125 V DC buses, respectively. Power for SSW Pump A is provided by Division $14160 \mathrm{~V}$ AC Bus 15AA. Power for SSW Pump B is provided by Division $24160 \mathrm{~V}$ AC Bus $16 \mathrm{AB}$. Power to SSW Pump $C$ is provided by Division 3 $480 \mathrm{~V}$ AC Bus 17B01. A simplified dependency diagram of the SSW system is provided in Figure E6. The major dependencies are indicated by the solid diamonds.

Each loop's normally closed motor-operated valves receive motive power from a $480 \mathrm{~V}$ AC source. Valves on Train A receive power from Division 1 . Valves on Train $B$ receive power from Division 2, and valves on Train $C$ receive power from Division 3. Upon receipt of a SSW system actuation signal, start signals are sent to all pumps, and all normally closed MOVs that need to open are demanded to do so.

The SSW system has diverse methods for actuation:

(1) The signal that actuates a front-line emergency system will actuate the system;

(2) A loss of offsite power will actuate the system; or

(3) The actuation of any pump that requires cooling from the SSW system will actuate the system.

Because of this diversity, the failure of the SSW to actuate was considered to be negligible compared to other system failures and was not modeled.

\section{E.3 High Pressure Core Spray System (HPCS)}

\section{E.3.1 HPCS System Description}

The function of the HPCS system is to provide coolant makeup to the reactor vessel in order to maintain proper water level and/or flood the reactor. The HPCS system consists of a single train with motor-operated valves and a motor driven pump. Suction is taken from either the Condensate Storage Tank (CST) or the suppression pool. Injection to the reactor vessel is via a spray ring mounted inside the core shroud. The pump is capable of delivering $550 \mathrm{gpm}$ against a reactor pressure of $1177 \mathrm{psig}$ and a full flow of $7115 \mathrm{gpm}$ against a reactor pressure of $200 \mathrm{psig}$. The total maximum pump run out flow is $9100 \mathrm{gpm}$. The HPCS pump is located in the auxiliary building at elevation 93' 0 " in an enclosed room complete with fire/flood doors. The simplified schematic of the HPCS is provided by
Figure E7. Major system components are represented with valves shown in their normal standby position.

The HPCS system is automatically initiated and controlled. However, operator intervention is required to throttle flow to prevent the HPCS injection valve from opening and closing in response to the reactor vessel level. The operator may also be required to manually start the system, if an automatic start failure occurs.

Most of the HPCS system is located in a separate room in the auxiliary building. Because of the relative location of the system components, local access to the HPCS system would not be affected by either containment venting or containment failure. Room cooling failure is assumed to fail the HPCS pump in twelve hours.

\section{E.3.2 HPCS System Interfaces and Dependencies}

The HPCS system major dependencies are DC control power for initiating the actuation relay logic and HPCS pump breaker, AC power for operating the HPCS pump and valves, and HPCS pump room cooling. A simplified dependency diagram of the HPCS system is provided in Figure E8. Shown are the major support requirements for the HPCS system as indicated by the solid diamonds at the appropriate locations.

The DC power is provided by Division $3125 \mathrm{~V}$ DC Bus 11 DC. Power for the HPCS pump is provided by Division 3 $4160 \mathrm{~V} \mathrm{AC}$ Bus $17 \mathrm{AC}$, and power for the valves and room cooler is provided by Division $3480 \mathrm{~V}$ AC MCC 17B01. It should be noted that Division 3 (AC and DC power) is dedicated to the HPCS system and its supports.

The HPCS and Reactor Core Isolation Cooling (RCIC) systems share a common CST suction valve. This is a normally open manual valve and is identified as XV70 on the HPCS schematic. Failure of this valve will fail the CST as a suction source to both HPCS and RCIC. Note that no credit was taken for the RCIC system in POS 5 since RCIC auto isolates at a reactor pressure of $60 \mathrm{psig}$. Upon system actuation, the HPCS injection valve receives a signal to open, and the HPCS test valves receive a signal to close. The HPCS system is automatically initiated on the receipt of either a high drywell pressure signal (2 psig) or low reactor water level (-42 inches or Level 2). A simplified actuation dependency diagram of the major emergency coolant actuation subsystems is provided by Figures E9 and E10. The CST is the initial suction source for the HPCS system. Suction is automatically switched to the suppression pool upon either low CST level or high suppression pool level. 

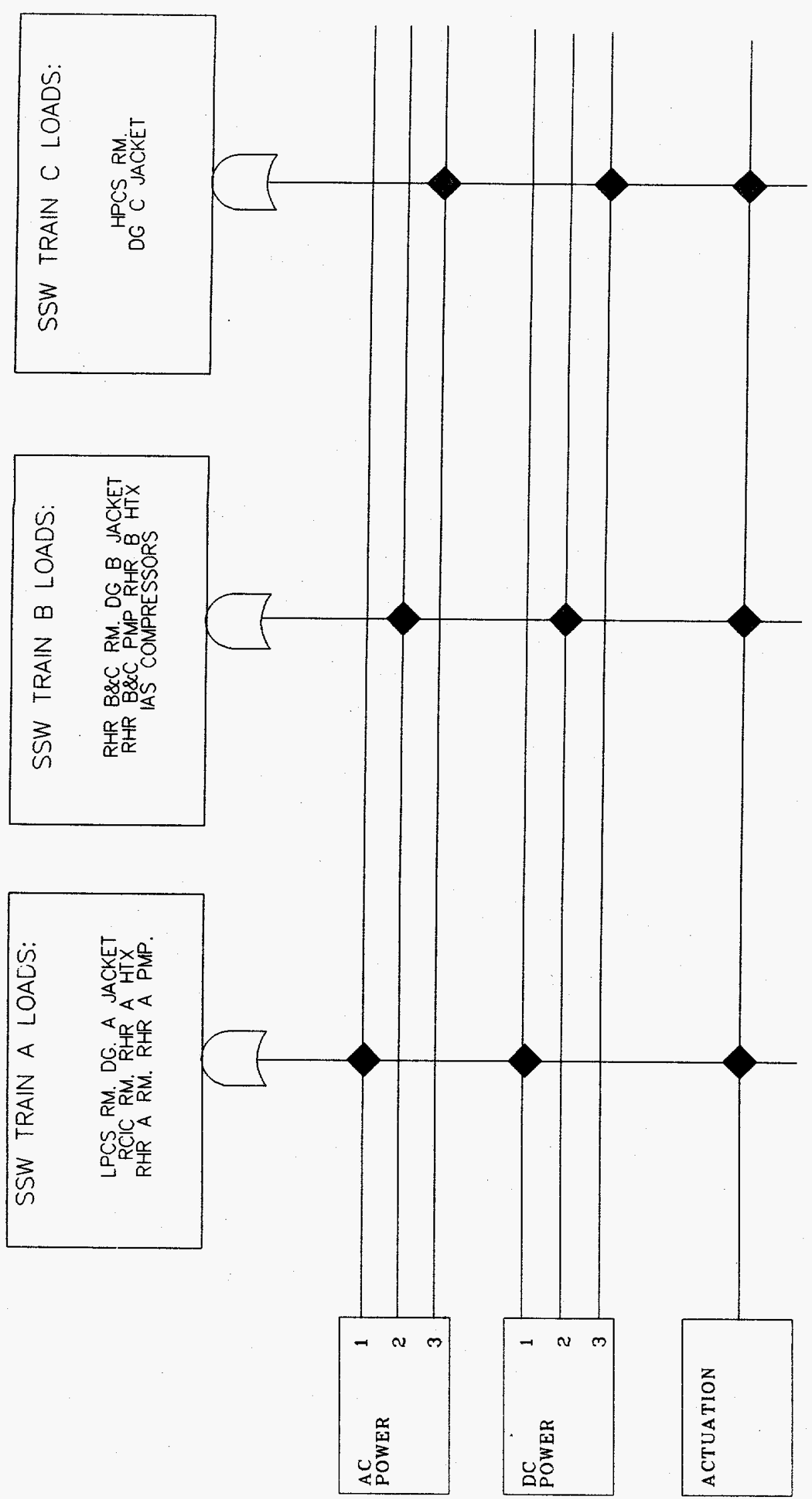

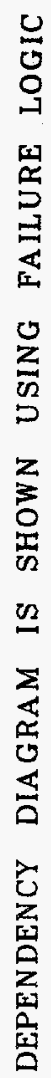

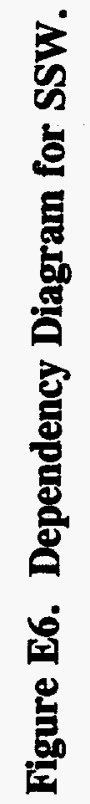


Appendix E

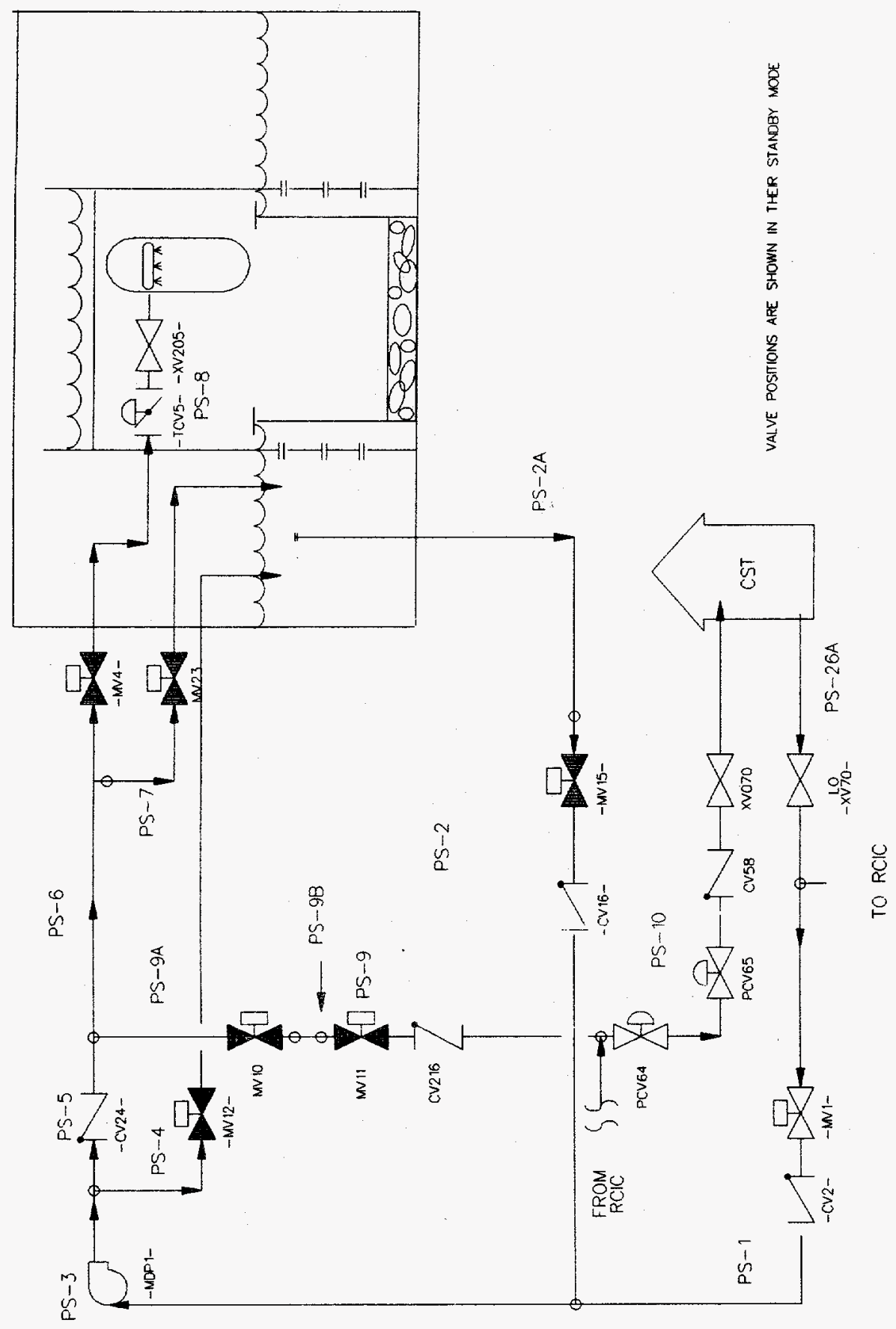

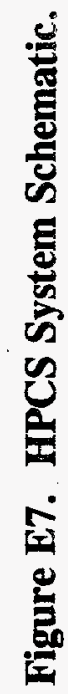


Appendix E

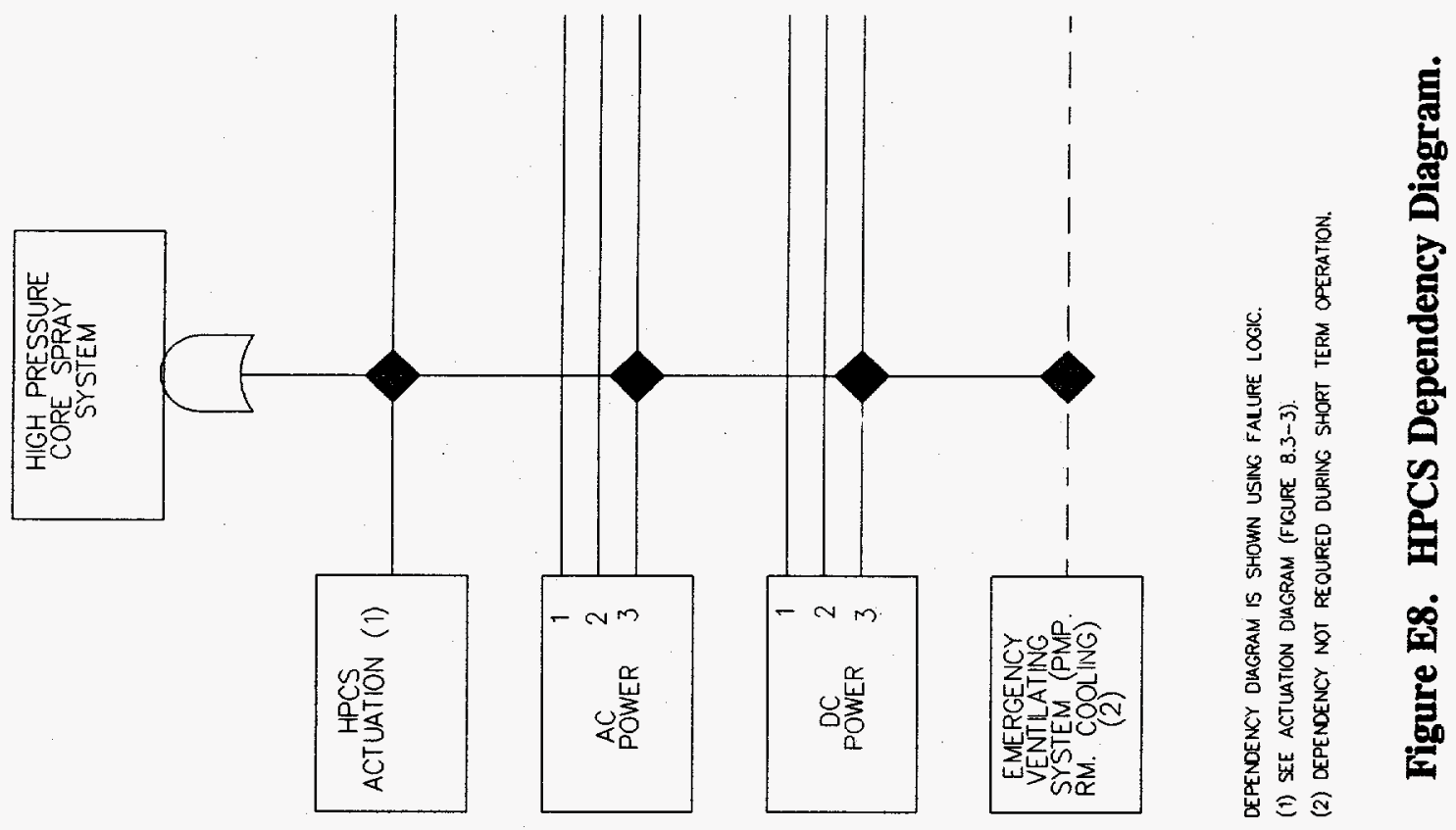




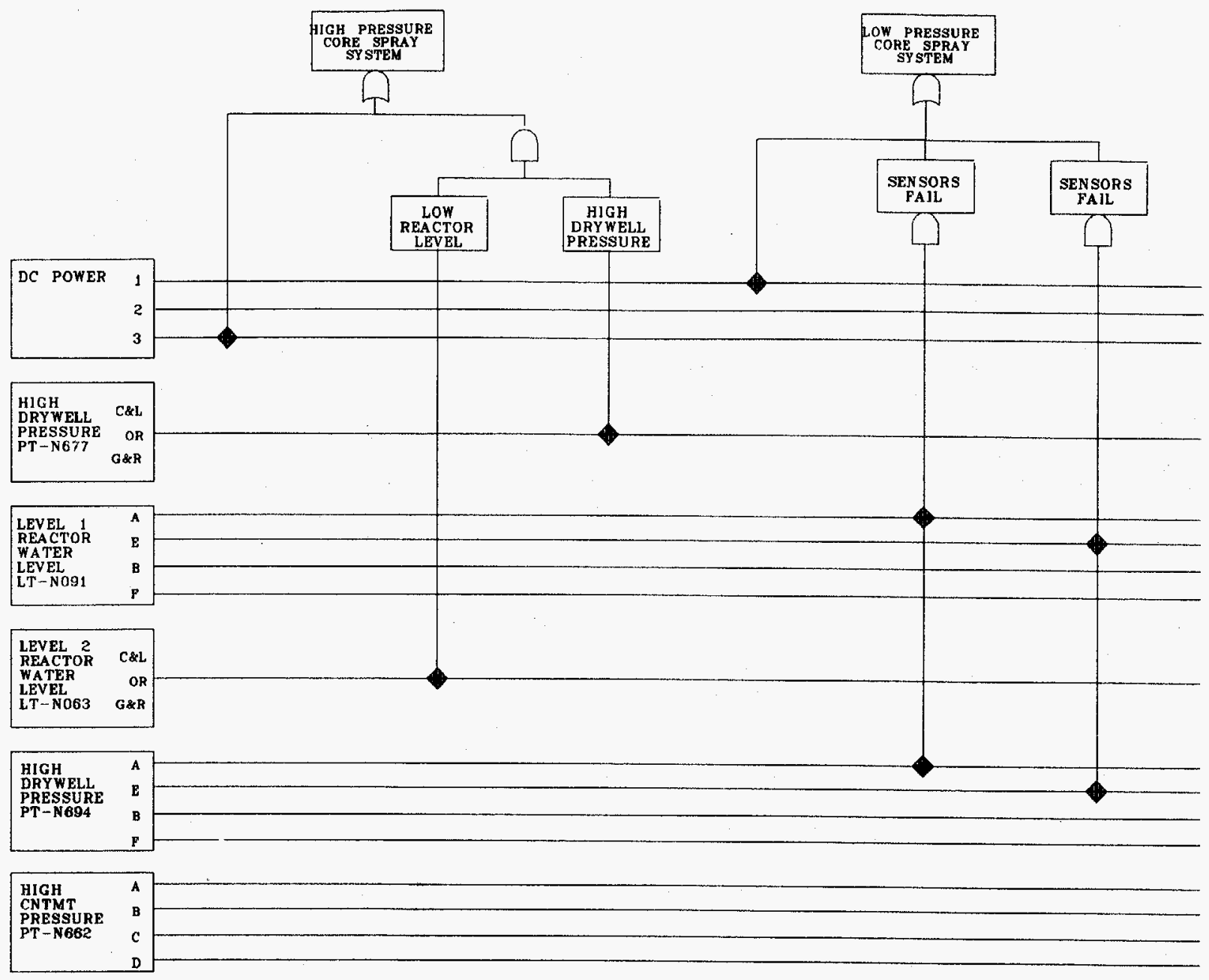

DEPENDENCY DIAGRAM IS SHOWN USING PAILURE LOGIC

Figure E9. System Actuation Dependency Diagram (page 1 of 2). 


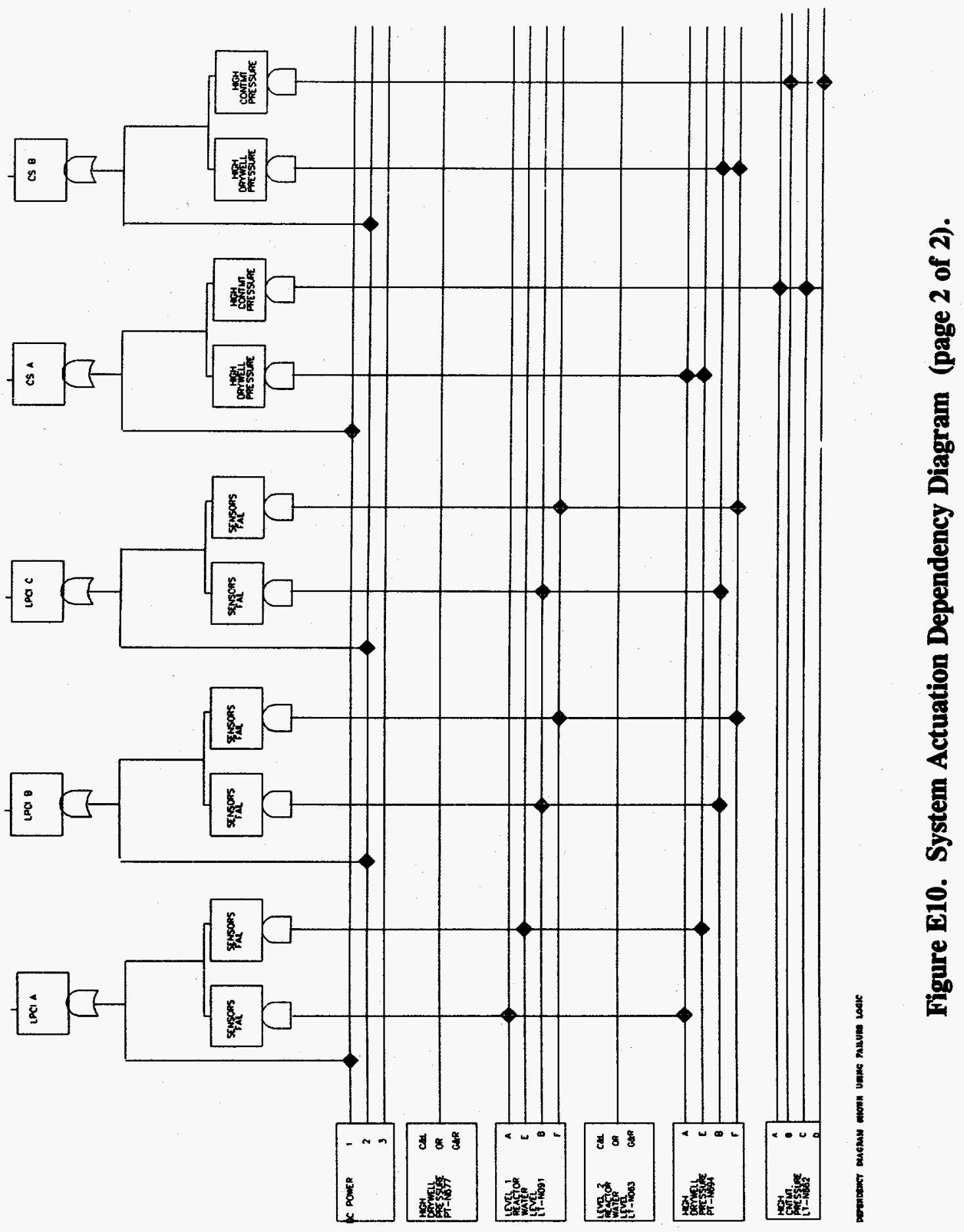


The CST suction valve closes when the suppression pool suction valve is fully open. This interlock prevents inadvertent draining of the CST to the suppression pool. Opening of the suppression pool valve will also result in closure of the CST test return line valves if they are open. This interlock prevents flow of suppression pool water to the CST. The HPCS system is automatically isolated when the reactor water level reaches +55 inches (Level 8). At this level, the HPCS injection valve closes and the minimum flow valve to the suppression pool opens. The HPCS pump continues to run.

\section{E.4 Low Pressure Core Spray (LPCS) System}

\section{E.4.1 LPCS System Description}

The function of the LPCS System is to provide coolant to the reactor vessel during accidents in which vessel pressure is low.

The LPCS system is a single train system consisting of motor-operated and manual valves and a motor-driven pump. The LPCS pump is rated at $7115 \mathrm{gpm}$ with a discharge head of $319 \mathrm{psig}$. The LPCS pump takes water from the suppression pool through strainers located 10 feet above the suppression pool floor. The LPCS pump is located in the auxiliary building at elevation $93^{\prime} 0^{\prime \prime}$ in an enclosed room with fire/flood doors. A simplified schematic of LPCS is provided by Figure E11. Major system components are shown with valves shown in their normal standby position.

The LPCS system is automatically initiated and controlled. The operator may be required to manually start the system if an automatic actuation failure occurs.

\section{E.4.2 LPCS Interfaces and Dependencies}

The LPCS system major dependencies are DC control power for initiating the actuation relay logic and LPCS pump breaker, AC power for operating the LPCS pump and valves, and LPCS pump room cooling.

The DC power is provided by Division 1125 V DC Panel 1E12-JB1. Power for the LPCS pump is provided by Division $14160 \mathrm{~V}$ AC Bus 15AA, and power for the valves is provided by Division $1480 \mathrm{~V} \mathrm{AC} \mathrm{MCC} \mathrm{15B11.} \mathrm{A}$ simplified dependency diagram of the LPCS system is provided in Figure E12. Major dependencies are indicated by the solid diamonds.
Upon the receipt of a LPCS injection signal, a start signal is sent to the LPCS pump, the injection valve is demanded to open, and the test return valve is demanded to close. The LPCS system is automatically initiated on the receipt of either a low reactor water level ( -150 inches) signal cr high drywell pressure (+2 psig) signal.

Most of the LPCS system is located in the auxiliary building. Because of the relative location of the system components, local access to the LPCS system would not be affected by either containment venting or containment failure. Room cooling failure is assumed to fail the LPCS pump in four hours.

\section{E.5 Low Pressure Coolant Injection (LPCI) System}

\section{E.5.1 LPCI System Description}

The function of the LPCI system is to provide coolant to the reactor vessel during accidents in which system pressure is low. The LPCI system is but one mode of the RHR system and, as such, shares components with other modes. The LPCI system is a three train system consisting of motor-operated valves and motor driven pumps. The three pumps are each rated at $7450 \mathrm{gpm}$. Trains A and B each have two heat exchangers in series downstream of the pump. Train $C$ is injection dedicated and has no heat exchangers. Cooling water flow to the heat exchangers is not required for the LPCI mode. The LPCI pump suction source is the suppression pool. The LPCI pumps are located in the auxiliary building at elevation $93^{\prime} 0^{\prime \prime}$ in an enclosed room with fire/flood doors. A simplified schematic of the LPCI system is provided in Figure E13. Major system components are shown with valves shown in their normal standby position.

The LPCI system is automatically initiated and controlled. However, operator intervention may be required to manually realign and start the system in POS 5 since the individual RHR Trains A and B could be aligned for shutdown cooling or ADHRS and Train $C$ could be aligned for ADHRS, given any of these configurations, the associated train would not automatically initiate for LPCI operation.

The success criterion for the LPCI system is injection of flow from any one pump to the reactor vessel. Most of the LPCI system is located in the auxiliary building. Because of the relative location of the system components, local access to the LPCI system would not be affected by either containment venting or containment failure. Room cooling failure is assumed to fail the RHR pumps in four hours. 
Appendix E

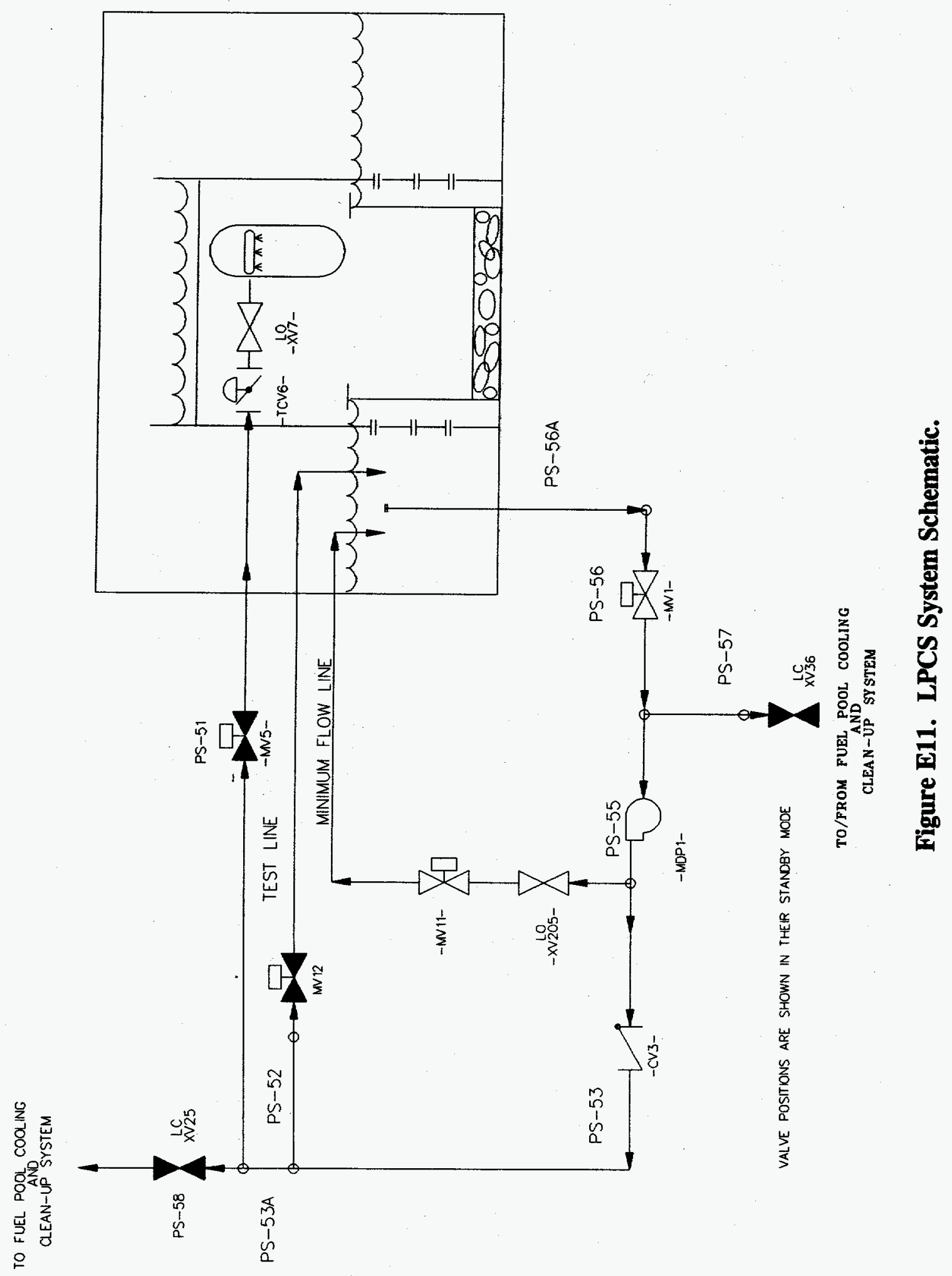


Appendix E

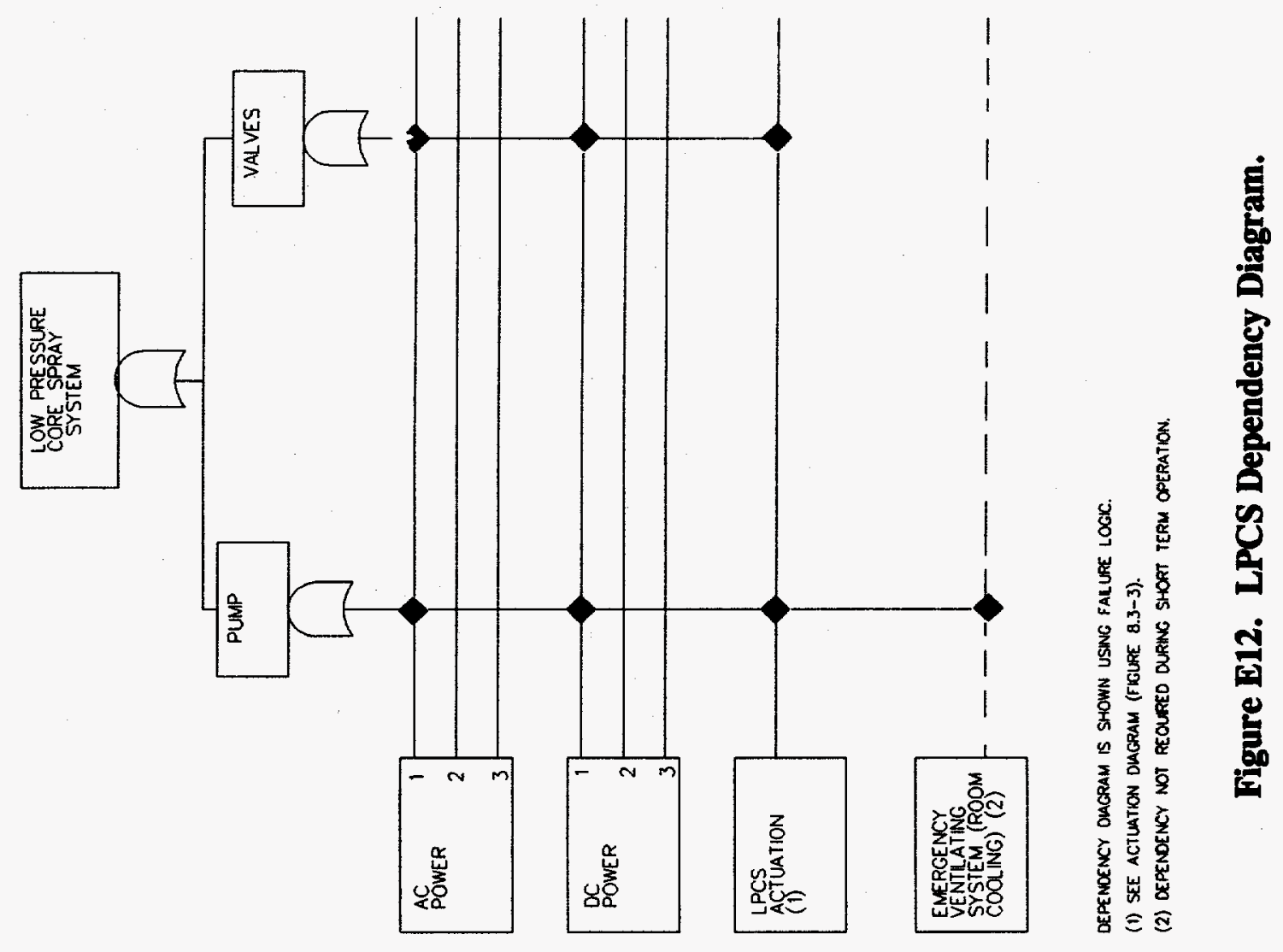




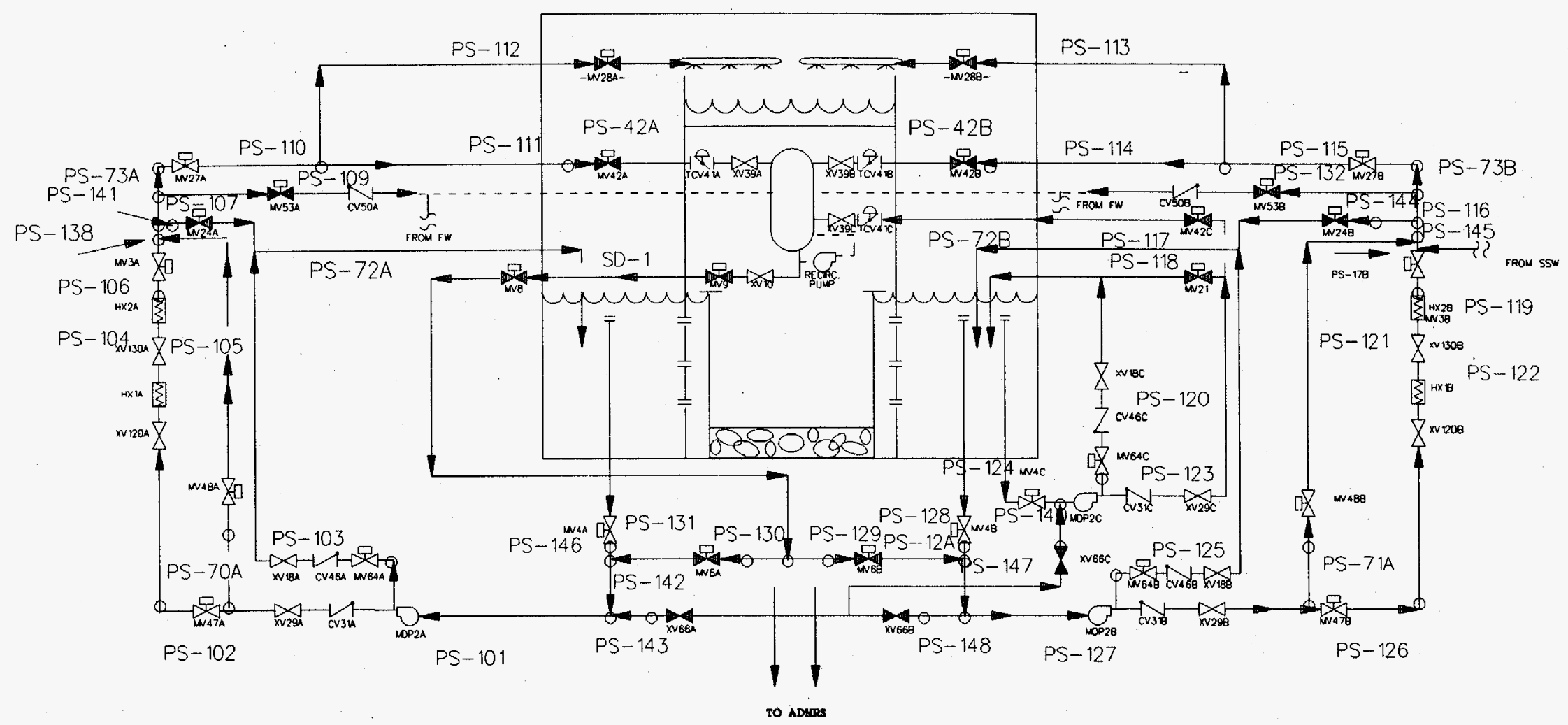

VALVE POSITIONS ARE SHOWN IN THER STANDBY MODE 


\section{E.5.2 LPCI Interfaces and Dependencies}

The LPCI system major dependencies are DC control power for initiating the actuation relay logic and RHR pump breakers, AC power for operating the RHR pumps and valves, RHR pump cooling, and RHR pump room cooling.

The DC power to Train A is provided by Division $1125 \mathrm{~V}$ DC; for Trains B and C, it is provided by Division $2125 \mathrm{~V}$ DC. Power for RHR Pump A is provided by Division 1 $4160 \mathrm{~V}$ AC Bus 15AA. Power for RHR Pump B and LPCI Pump C is provided by Division $24160 \mathrm{~V}$ AC Bus $15 \mathrm{AB}$. All pumps require pump cooling. A simplified dependency diagram of the LPCI system is provided by Figure E14. The major dependencies are indicated by the solid diamonds.

Each normally closed injection valve receives motive power from a $480 \mathrm{~V}$ AC source. Train A injection valve's source is the Division $1480 \mathrm{~V} \mathrm{AC}$ Bus $15 \mathrm{~B} 31$ and Trains B and C injection valves' source is the Division $2480 \mathrm{~V}$ AC Bus 16B31. Many components of the LPCI system are shared with the different modes of the RHR system. These commonalities are as follows:

(1) RHR pumps A and B are common to LPCI, Suppression Pool Cooling (SPC), Shutdown Cooling (SDC), and Containment Spray (CS) modes; and

(2) the suppression pool suction valve for Pump Trains A and B is common to the LPCI, SPC, and CS modes.

Upon receipt of a LPCI injection signal, start signals are sent to all pumps. Trains $A, B$ and $C$ injection valves are demanded to open. The test return valves are demanded to close. The LPCI system is automatically initiated on the receipt of either a low reactor water level signal (-150 inches) or a high drywell pressure signal ( $+2 \mathrm{psig}$ ).

Train A actuation sensors for LPCI actuation and control circuitry are divided into two divisions. Division $A$ is associated with the actuation and control of components in Loop A, and Division B is associated with the actuation and control of components in Loops B and C.

\section{E.6 Alternate Decay Heat Removal (ADHR) System}

\section{E.6.1 ADHR System Description}

The function of the ADHR system is to provide an alternate method of decay heat removal during cold shutdown and refueling when maintenance is being performed on the RHR shutdown cooling loops or associated support systems. The functional purpose of the ADHR system is important to safety, but is not safety related since the ADHR does not automatically mitigate the consequences resulting from accidents.

The ADHR system consists of components common to the RHR system including the RHR common suction line, fuel pool cooling and cleanup piping, and the RHR Train C LPCI injection header. Components exclusive to ADHR include 2 ADHR pumps, 2 heat exchangers, associated piping, valves, and instrumentation and controls.

ADHR can operate in four main modes of which one is modeled for POS 5. In POS 5, ADHR is used in the reactor vessel cooling mode via RHR A or B. During the reactor vessel cooling mode, ADHR draws water from the existing RHR common suction line. The reactor coolant is then pumped from the reactor recirculation loop through valves FO66A and F0O6A or valves F066 and F006B to the ADHR pumps, then to the heat exchangers and back to the reactor vessel via RHR C LPCI injection line. A schematic of the ADHR system is shown in Figure E15. Major system components are shown with valves in their normal standby positions. Most of the ADHR system is located in the auxiliary building.

Control for the ADHR system is remote manual from the control room. Flow and temperature indications are provided in the control room for ADHR heat exchangers while individual manual control of pump operation with pump running status lights is provided.

The success criterion for the ADHR system is to provide cooling to the reactor vessel at rated flow. 


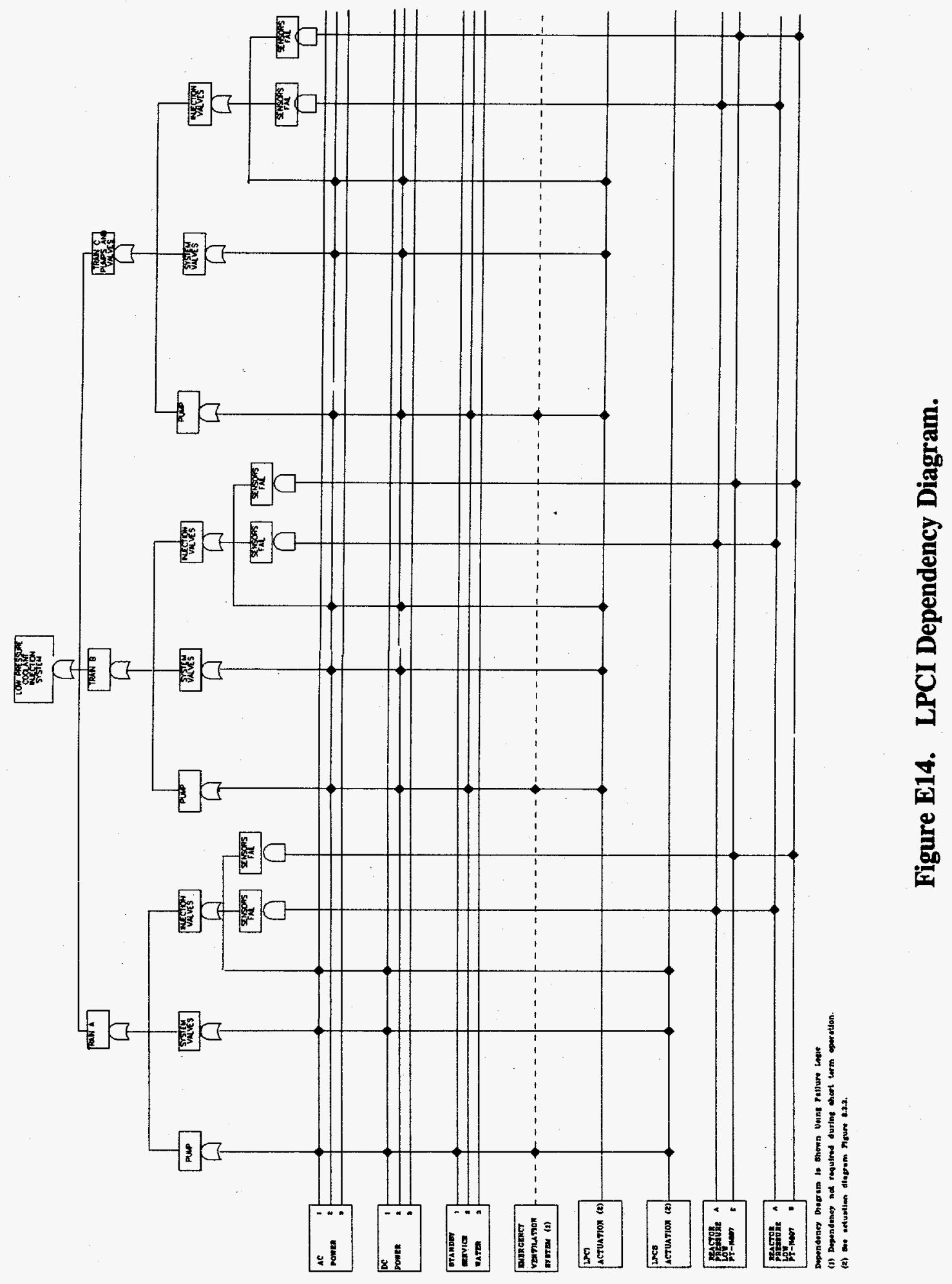




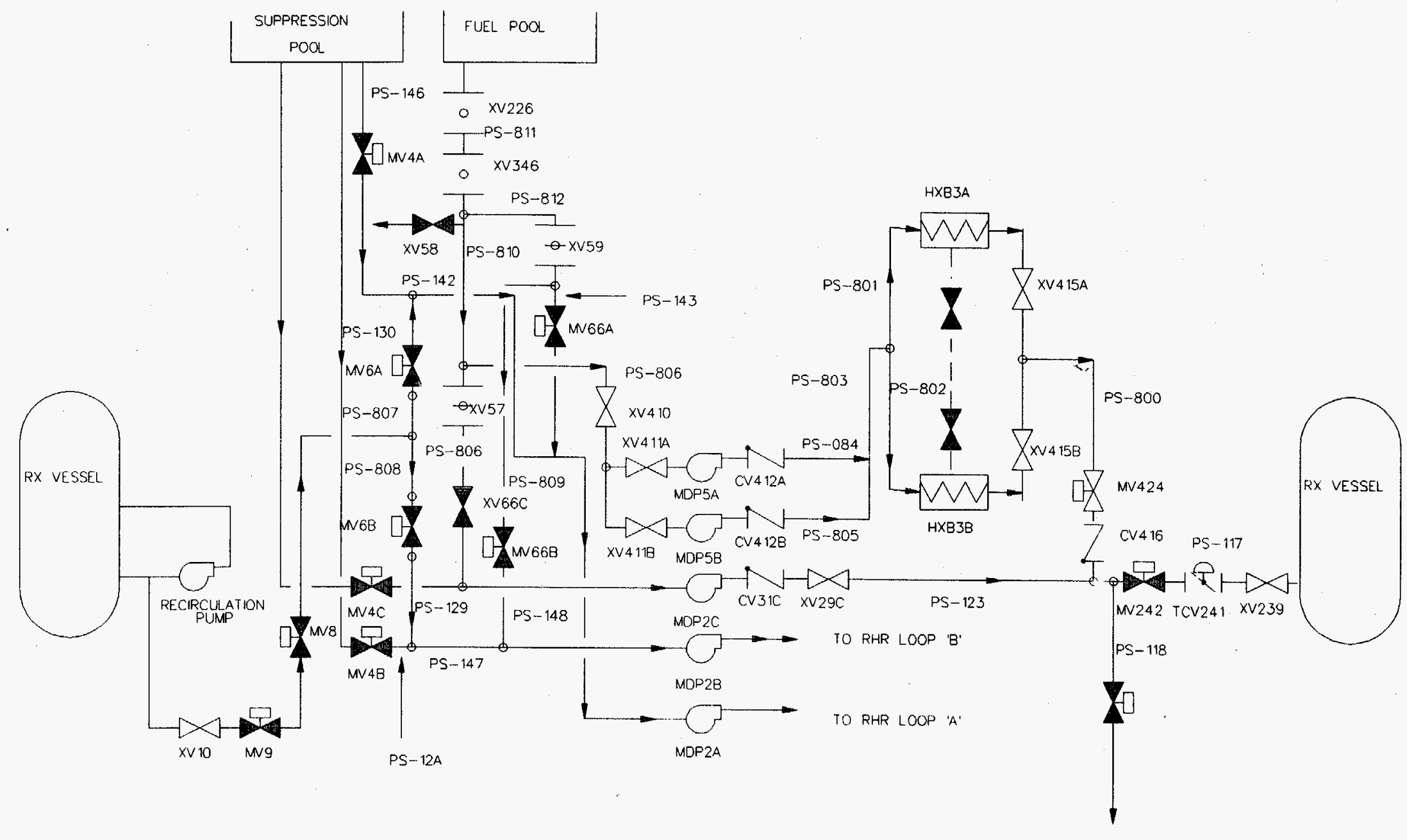




\section{E.6.2 ADHR Interfaces and Dependencies}

The ADHR major dependencies are AC Division 1 and 2 power for motor operated valves F066A and F066B respectively, and BOP AC bus 14HE for the ADHR pumps and motor operated valve F424. Plant Service Water (PSW) cools the heat exchangers. Major dependencies are indicated by solid diamonds in Figure E16.

\section{E.7 Safety Relief Valves}

\section{E.7.1 SRV Description}

The Safety Relief Valves (SRVs) are designed to prevent reactor vessel overpressurization which could lead to the failure of the reactor coolant pressure boundary. Also, eight of the SRVs are used by the Automatic Depressurization System (ADS) to depressurize the reactor vessel to a pressure at which the low pressure injection systems can inject coolant to the reactor vessel.

There are twenty SRVs (eight of these are also ADS valves) that operate in a safety mode and a relief mode. The safety mode (or spring actuated mode) of operation consists of direct action of the reactor vessel steam pressure against a spring loaded disk that will pop open when the valve inlet pressure force exceeds the spring force. The safety mode of operation is a backup to the relief mode of operation. The relief mode (or power actuated mode) of operation consists of using a pneumatic piston/cylinder assembly which opens the valve by overcoming the spring force, even with valve inlet pressure equal to zero psig. Each valve has a pressure sensing device which operates at designated set points. When the set pressure is reached, the pressure sensing device operates a solenoid air valve which in turn actuates the pneumatic piston/cylinder to open the valve.

There are two solenoids per SRV, one is powered from DC Division I and the other is powered from
DC Division II. Either solenoid can operate the air valve. All twenty SRVs can be operated in the relief mode (power actuated mode) by remote-manual controls from the main control room. The pneumatic operator is arranged so that if it malfunctions, it will not prevent the safety mode of operation.

Relief valve capacity is approximately $900,000 \mathrm{lb} / \mathrm{hr}$. Each SRV discharges steam from the main steam line through discharge piping to a point below the minimum suppression pool water level of $18^{\prime} 41 / 12^{\prime \prime}$. A simplified schematic of the SRVs including the ADS function is provided in Figure E17.

The SRVs are located inside the drywell. Containment pressures of 100 psi will prevent the opening of the SRVs. This scenario is not a consideration in the current study because containment is assumed to have failed at $60 \mathrm{psig}$. Therefore, it is assumed that containment conditions will not affect SRV performance.

\section{E.7.2 SRV Interfaces and Dependencies}

The SRVs depend upon the Instrument Air System (IAS) and two $125 \mathrm{~V}$ DC power sources. One of the solenoids in each SRV that can actuate the air valve is powered by Division I $125 \mathrm{~V}$ DC bus 11DA, and the other is powered by Division II 125 V DC bus 11DB. The IAS supplies air pressure to open the SRVs.

Accumulators for each SRV contain sufficient air for one actuation if the IAS is unavailable; ADS accumulators contain sufficient air for two actuations. A simplified dependency diagram of the SRVs including the ADS function is provided in Figure E18. The major dependencies are indicated by the solid diamonds. 


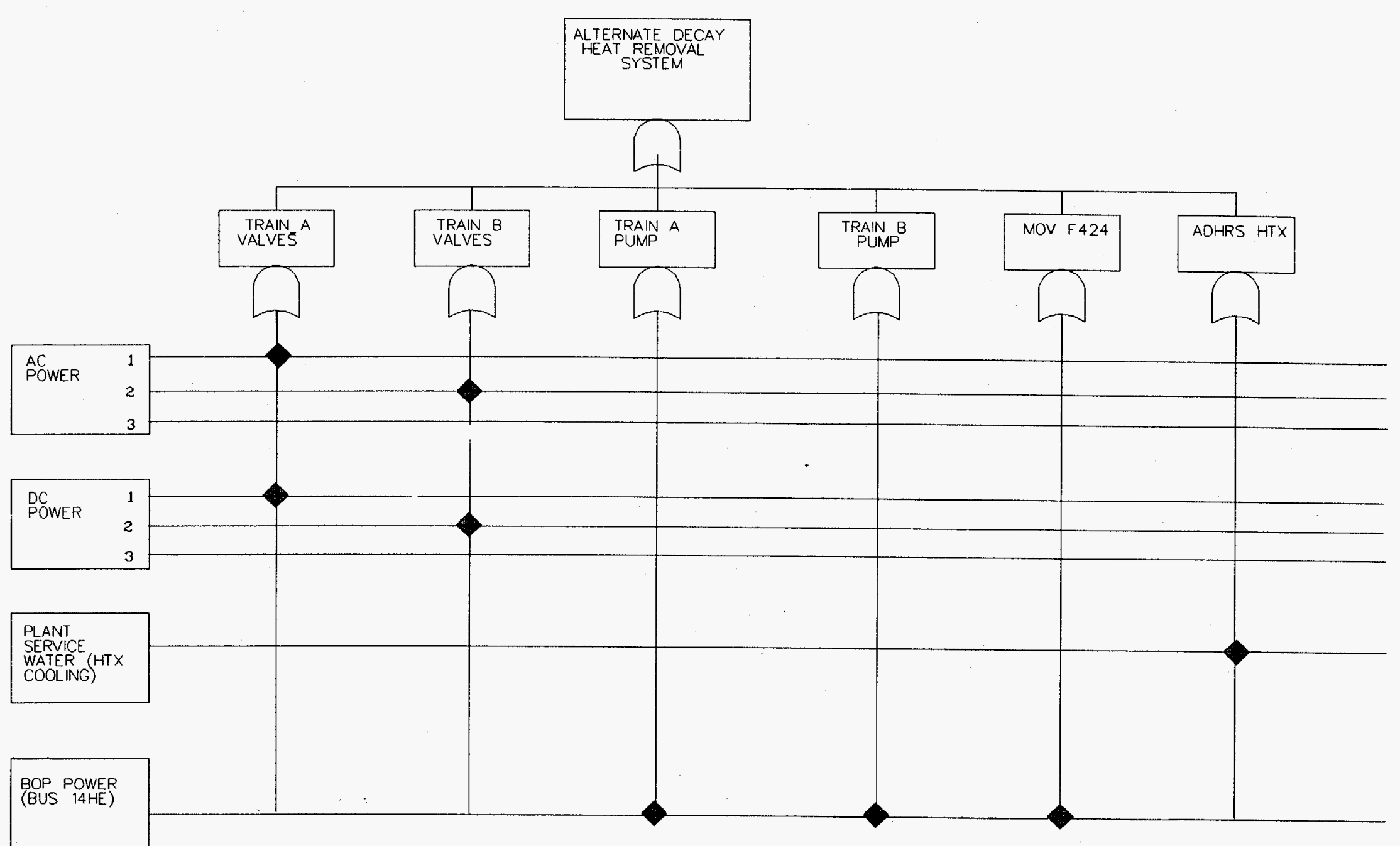




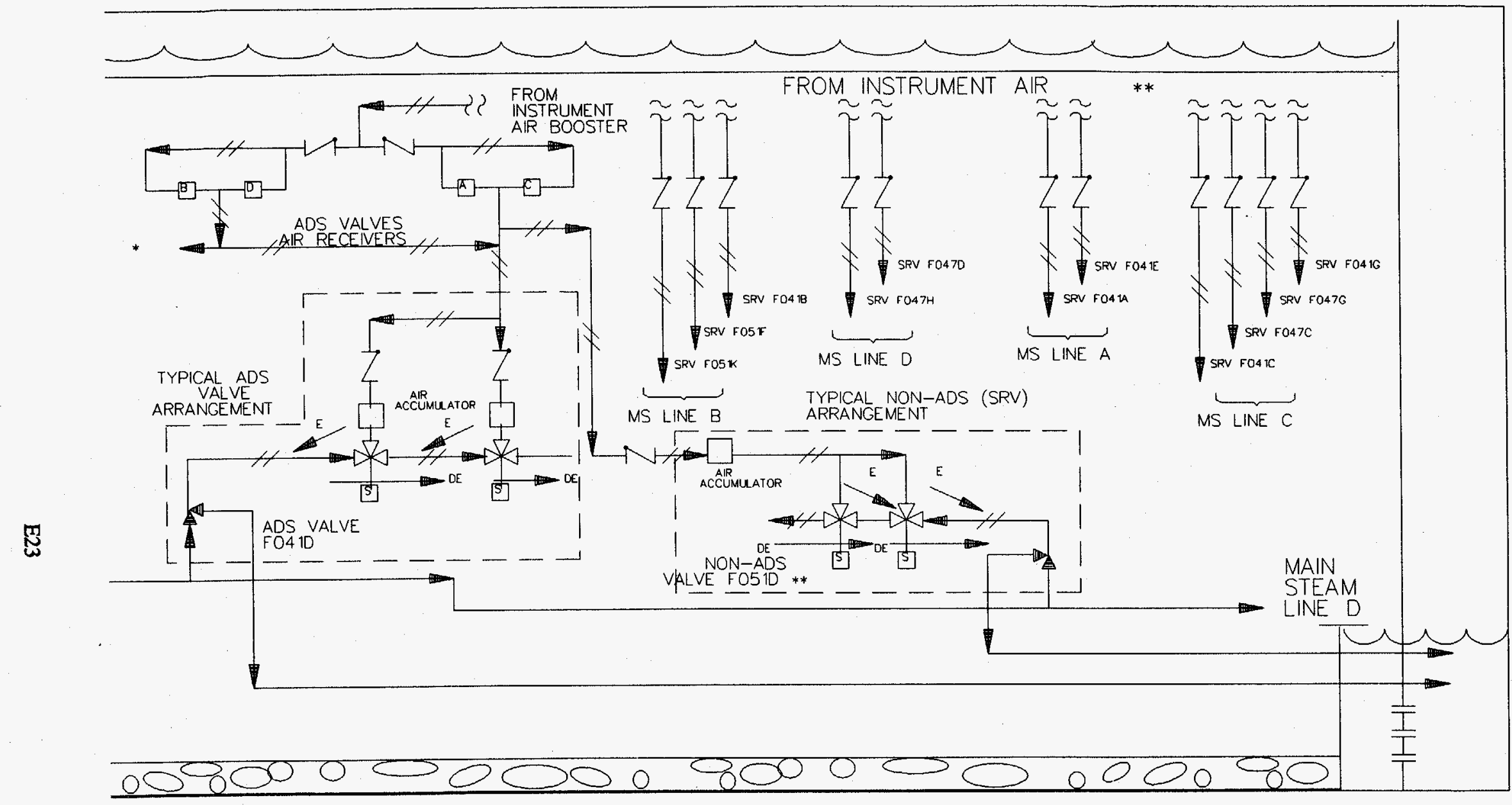

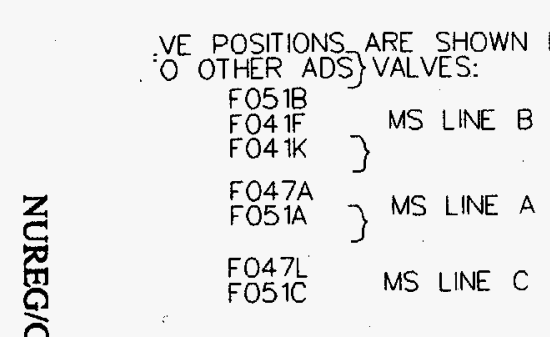

**NON-ADS SRV F051D RECEIVES ITS AIR SUPPLY FROM THE ADS VALVES AIR RECEIVERS AND THE INSTRUMENT AIR BOOSTER, THE REMAINING SRVS, ALTHOUC SAME ARRANGEMENT AS SRV FR FROM THE INSTRUMENT AIR SUPPLY SYSTEM
E. ENERGIZED MS..MAINSTEAM

Figure E17. ADS and SRV System Schematic. 
Appendix E

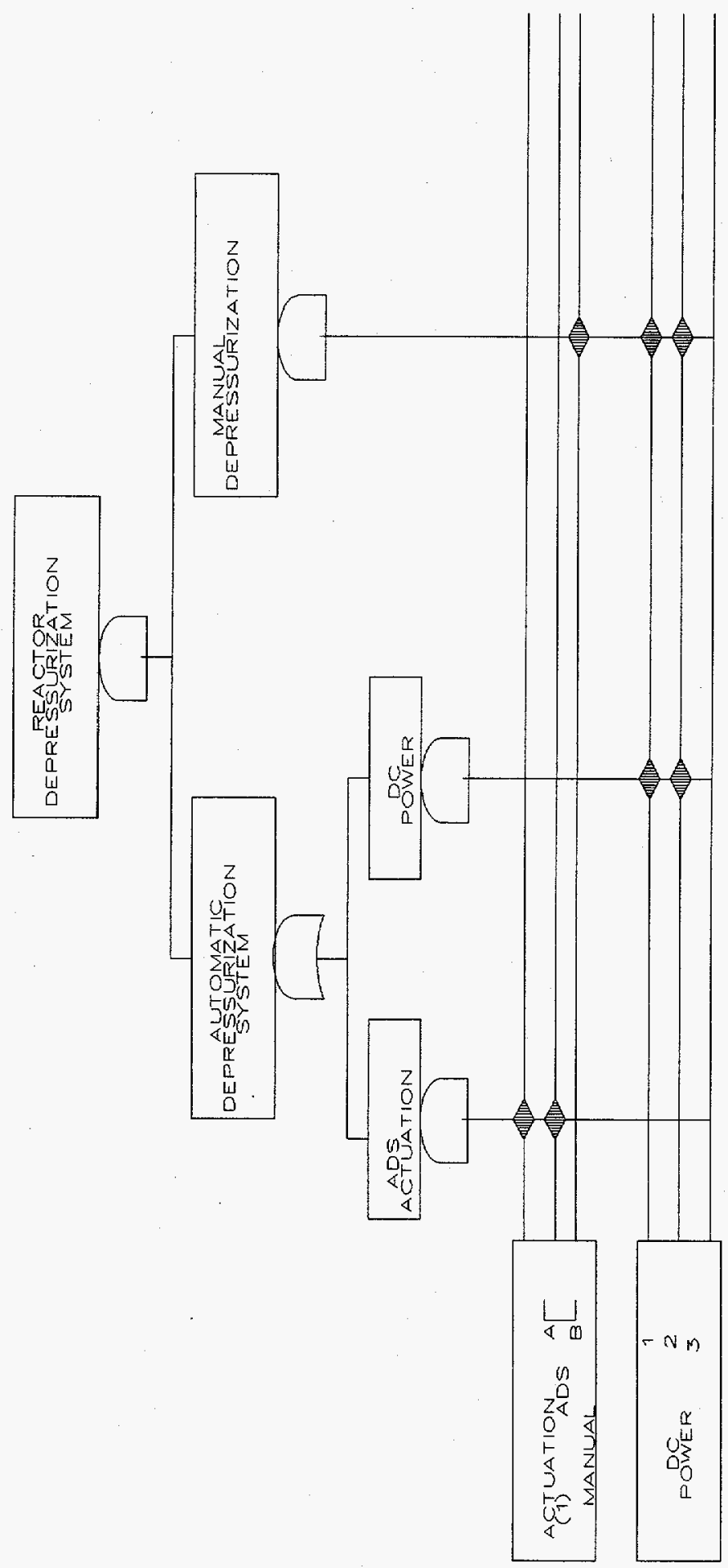

㤩 


\section{DISTRIBUTION}

\section{External}

C. E. Johnson, RES/HFB (20)

Mail Stop T-10 E33

U.S. Nuclear Regulatory Commission

Internal

$\begin{array}{ll}\text { MS0747 } & \text { A. L. Camp, 6412 } \\ \text { MS0747 } & \text { V. J. Dandini, 6412 } \\ \text { MS0747 } & \text { S. L. Daniel, 6412 } \\ \text { MS0747 } & \text { S. E. Dingman, 6412 } \\ \text { MS0747 } & \text { J. A. Forester, 6412 } \\ \text { MS0747 } & \text { K. M. Hays, 6412 } \\ \text { MS0747 } & \text { D. B. Mitchell, 6412 } \\ \text { MS0747 } & \text { B. D. Staple, 6412 (25) } \\ \text { MS0747 } & \text { G. D. Wyss, 6412 } \\ \text { MS0747 } & \text { D. W. Whitehead, 6412 } \\ \text { MS0744 } & \text { W. A. von Riesemann, 6403 } \\ \text { MS0744 } & \text { D. A. Powers, 6404 } \\ \text { MS0744 } & \text { N. R. Ortiz (Acting), 6405 } \\ \text { MS0748 } & \text { F. T. Harper, 6413 } \\ \text { MS0742 } & \text { J. E. Kelly, 6414 } \\ \text { MS0745 } & \text { S. L. Thompson, 6418 } \\ \text { MS9018 } & \text { Central Technical Files, 8523-2 } \\ \text { MS0899 } & \text { Technical Library, 7141 (5) } \\ \text { MS0619 } & \text { Technical Publications, 7151 }\end{array}$


ISee instructions on the reverse)

NUREG/CR-6166

2. TITLE AND SUBTITLE

Risk Impact of Technical Specifications Requirements During Shutdown for BWRs

\section{AUTHOR(S)}

B. D. Staple, SNL

H. K. Kirk, SNL

J. Yakle, SAIC

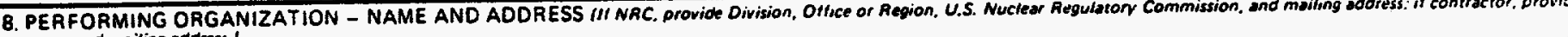
name and moiling wodress.

Risk Assessment and Systems Modeling

Department 6412

Sandia National Laboratories

Albuquerque, NM 87185

9. SPONSORING ORGANIZATION - NAME AND ADDRESS III NRC, sype "Some as soove"; if contractor, provide NAC Division, Office or Region. U.S. Nuclear Regulasory Commissuon. and mailing oddress,

Division of Systems Research

Office of Nuclear. Regulatory Research

U.S. Nuclear Regulatory Commission

Washington, DC 20555-0001

10. SUPPLEMENTARY NOTES

11. ABSTRACT 1200 words or kessi

This report presents an application of probabilistic models and risk based criteria for determining the risk impact of the Limiting Conditions of Operations (LCOs) in the Technical Specifications (TSs) of a boiling water reactor during shutdown. This analysis studied the risk impact of the current requirements of Allowed Outage

Times (AOTs) and Surveillance Test Intervals (STIs) in eight Plant Operational States (POSs) which encompass power operations, shutdown, and refueling. This report also discusses insights concerning TS action statements. 